\title{
Pd-Catalyzed Alkene Difunctionalization Reactions of Malonate Nucleophiles. Synthesis of Substituted Cyclopentanes via Alkene Aryl-Alkylation and Akenyl-Alkylation.
}

Derick R. White, Elsa M. Hinds, Evan C. Bornowski, and John P. Wolfe*

Department of Chemistry, University of Michigan, 930 N. University Avenue, Ann Arbor, Michigan 48109-1055

\section{Supporting Information}

General Considerations

S1

Preparation and Characterization of Substrates

S2

Preparation and Characterization of Products

S14

Optimization Studies for Reactions of Internal Alkene Substrate 1j

S32

Descriptions of Stereochemical Assignments

S32

References

S39

Copies of NMR Spectra

S40

General Considerations: All reactions were carried out under a nitrogen atmosphere in flamedried glassware. All reagents, palladium precatalysts, and ligands were purchased from commercial sources and were used without purification unless otherwise noted. The substrates 1a-e, ${ }^{1} \mathbf{1 f},{ }^{2}$ 4a, ${ }^{3}$ 4c, ${ }^{3}$ 4d, $,{ }^{1} \mathbf{7},{ }^{1}$ (Brettphos)Pd(allyl)(Cl), ${ }^{4} \mathrm{~N}$-(2-pyridyl)triflimide, ${ }^{5}$ and 4-tert-butyl-2alllylphenol $^{6}$ were prepared by previously published methods. Alkenyl triflate starting materials were stored in a freezer under nitrogen. Bulk quantities of cesium carbonate, lithium tertbutoxide, and lithium hexamethyldisilazide were stored in nitrogen-filled glove box and small 
amounts were removed within a few days of use. Toluene, THF, dichloromethane, and diethyl ether were purified using a GlassContour solvent purification system. Anhydrous dioxane was purchased from Sigma-Aldrich and was used without purification. Structural and stereochemical assignments were made on the basis of 2-D COSY and NOESY experiments. Ratios of diastereomers were determined by ${ }^{1} \mathrm{H}$ NMR analysis. Yields refer to isolated yields of compounds estimated to be $\geq 95 \%$ pure as determined by ${ }^{1} \mathrm{H}$ NMR analysis unless otherwise noted. The yields reported in the supporting information describe the result of a single experiment, whereas yields reported in Schemes 2-4 and equations 3-4 are average yields of two or more experiments. Thus, the yields reported in the supporting information may differ from those shown in Schemes 2-4 and equations 3-4.

\section{Preparation and Characterization of Substrates}<smiles>C=CCc1cc(C(C)(C)C)ccc1OCC</smiles>

2-Allyl-4-(tert-butyl)phenyl trifluoromethanesulfonate (4b). A flame-dried flask equipped with a stir bar was cooled under a stream of nitrogen and charged with 2-allyl-4-tert-butylphenol ${ }^{6}$ (0.231 g, $1.21 \mathrm{mmol}, 1.0$ equiv) and dichloromethane $(1.3 \mathrm{~mL}, 1 \mathrm{M})$. Pyridine $(0.117 \mathrm{~mL}, 1.48$ mmol, 1.2 equiv) was added, and the reaction was cooled to $0{ }^{\circ} \mathrm{C}$. Trifluoromethanesulfonic anhydride $(0.225 \mathrm{~mL}, 1.34 \mathrm{mmol}, 1.1$ equiv) was added, the ice bath was removed, and the reaction was stirred for $15 \mathrm{~h}$ at $\mathrm{rt}$. The mixture was quenched with ammonium chloride and extracted with $\mathrm{DCM}(\mathrm{x} 3)$. The organic layer was dried with $\mathrm{MgSO}_{4}$ and concentrated in vacuo to yield a brown oil. The crude material was purified via column chromatography on silica gel using 99:1 hexanes:ethyl acetate as the eluent. This procedure afforded $0.334 \mathrm{~g}(85 \%)$ of the title compound as a colorless oil. ${ }^{1} \mathrm{H}$ NMR $\left(500 \mathrm{MHz}, \mathrm{CDCl}_{3}\right) \quad 7.32-7.25(\mathrm{~m}, 2 \mathrm{H}), 7.19-7.14(\mathrm{~m}$, 1H), $5.98-5.87(\mathrm{~m}, 1 \mathrm{H}), 5.18-5.09(\mathrm{~m}, 2 \mathrm{H}), 3.47(\mathrm{~d}, J=6.6 \mathrm{~Hz}, 2 \mathrm{H}), 1.31(\mathrm{~s}, 9 \mathrm{H}) .{ }^{13} \mathrm{C}$ NMR $\left(126 \mathrm{MHz}, \mathrm{CDCl}_{3}\right) \delta 151.5,145.7,134.9,131.9,128.3,125.1,120.7,118.5\left(\mathrm{q}, J_{\mathrm{C}-\mathrm{F}}=321 \mathrm{~Hz}\right)$ 
117.2, 77.2, 34.7, 34.3, 31.2. IR (film) 2967, $1491 \mathrm{~cm}^{-1}$. HRMS (ESI TOF) m/z: $\left[M+\mathrm{H}^{+}\right]^{+}$calcd for $\mathrm{C}_{14} \mathrm{H}_{17} \mathrm{~F}_{3} \mathrm{O}_{3} \mathrm{~S} 323.0850$; found 323.0565<smiles>Oc1ccccc1C/C=C/c1ccccc1</smiles>

2-Cinnamylphenol (S1). A flame-dried 2-neck flask equipped with a stir bar and a condenser was cooled under a stream of nitrogen and charged with phenol (2.0 g, $21.2 \mathrm{mmol}, 1.0$ equiv) and diethyl ether $(22 \mathrm{~mL}, 0.1 \mathrm{M})$. Sodium hydride $(60 \%$ in mineral oil, $1.7 \mathrm{~g}, 42.4 \mathrm{mmol}, 2.0$ equiv) was added, and the reaction stirred at $\mathrm{rt}$ for $30 \mathrm{~min}$. Cinnamyl chloride was added and the reaction heated to $37^{\circ} \mathrm{C}$ with stirring for $6 \mathrm{~h}$. The reaction was cooled to rt, and the mixture was transferred to an Erlenmeyer flask containing aqueous $\mathrm{HCl}(0.1 \mathrm{M}, 75 \mathrm{~mL})$. The mixture was stirred briefly then transferred to a separatory funnel. The layers were separated, and the aqueous layer was extracted with diethyl ether $(3 \times 20 \mathrm{~mL})$. The combined organic layers were washed with brine, dried over $\mathrm{MgSO}_{4}$, filtered and concentrated in vacuo to yield a yellow oil. The crude material was purified via column chromatography on silica gel using 95:5 -> 90:10 hexanes:ethyl acetate as the eluent. This procedure afforded $2.52 \mathrm{~g}(57 \%)$ of the title compound as a yellow semisolid, mp 57-58 ${ }^{\circ} \mathrm{C} .{ }^{1} \mathrm{H}$ NMR $\left(500 \mathrm{MHz}, \mathrm{CDCl}_{3}\right) \delta 7.28(\mathrm{~d}, \mathrm{~J}=18.1 \mathrm{~Hz}, 4 \mathrm{H}), 7.25$ - $7.11(\mathrm{~m}, 3 \mathrm{H}), 6.91(\mathrm{~s}, 1 \mathrm{H}), 6.82(\mathrm{~d}, J=8.0 \mathrm{~Hz}, 1 \mathrm{H}), 6.51(\mathrm{~d}, J=15.9 \mathrm{~Hz}, 1 \mathrm{H}), 6.44-6.34(\mathrm{~m}$, 1H), $4.89(\mathrm{~s}, 1 \mathrm{H}), 3.58(\mathrm{~d}, J=6.5 \mathrm{~Hz}, 2 \mathrm{H}) .{ }^{13} \mathrm{C}$ NMR $\left(126 \mathrm{MHz}, \mathrm{CDCl}_{3}\right) \delta$ 154.0, 137.1, 131.5, 130.5, 128.5 (2 peaks), 127.9 (2 peaks), 127.3, 126.2, 121.0, 115.8, 34.1. IR (film) 3531.5, 1591.7, $\mathrm{cm}^{-1}$. HRMS (ESI- TOF) m/z: [M - $\left.\mathrm{H}^{+}\right]^{-}$calcd for $\mathrm{C}_{15} \mathrm{H}_{14} \mathrm{O}$ 209.0966; found 209.0972.<smiles>CCCc1ccccc1C/C=C/c1ccccc1</smiles>

2-Cinnamylphenyl trifluoromethanesulfonate (4e). A flame-dried flask equipped with a stir bar was cooled under a stream of nitrogen and charged with 2-cinnamylphenol (S1) (0.934 g, $4.45 \mathrm{mmol}, 1.0$ equiv) and dichloromethane (20 mL, 0.2 M). Pyridine (0.72 mL, $8.9 \mathrm{mmol}, 2.0$ 
equiv) was added, and the reaction was cooled to $0^{\circ} \mathrm{C}$. Trifluoromethanesulfonic anhydride $(1.5$ $\mathrm{mL}, 8.9 \mathrm{mmol}, 2.0$ equiv) was added, the ice bath was removed, and the reaction was stirred for $15 \mathrm{~h}$ at $\mathrm{rt}$. The purple mixture was filtered through a pad of celite, eluting with ethyl acetate. The purple filtrate was concentrated in vacuo to yield a purple oil. The crude material was purified via column chromatography on silica gel using 98:2 hexanes:ethyl acetate as the eluent. This procedure afforded $1.18 \mathrm{~g}(78 \%)$ of the title compound as a colorless oil. ${ }^{1} \mathrm{H} \mathrm{NMR}(500 \mathrm{MHz}$, $\left.\mathrm{CDCl}_{3}\right) \delta 7.46-7.13(\mathrm{~m}, 9 \mathrm{H}), 6.51(\mathrm{~d}, J=15.8 \mathrm{~Hz}, 1 \mathrm{H}), 6.35-6.22(\mathrm{~m}, 1 \mathrm{H}), 3.65(\mathrm{~d}, J=6.8 \mathrm{~Hz}$, 2H). ${ }^{13} \mathrm{C}$ NMR $\left(126 \mathrm{MHz}, \mathrm{CDCl}_{3}\right) \delta 147.9,137.0,133.1,132.7,131.4,128.5,128.2,127.4$, 126.2, 126.1, 121.4, $118.5\left(\mathrm{q}, J_{\mathrm{C}-\mathrm{F}}=319 \mathrm{~Hz}\right), 33.2,29.7 . \mathrm{IR}$ (film) 1595.3, $\mathrm{cm}^{-1}$. HRMS (ESI TOF) $\mathrm{m} / \mathrm{z}:\left[\mathrm{M}+\mathrm{H}^{+}\right]^{+}$calcd for $\mathrm{C}_{16} \mathrm{H}_{13} \mathrm{~F}_{3} \mathrm{O}_{3} \mathrm{~S}$ 342.0538; found 342.0538.

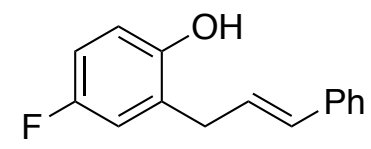

2-Cinnamyl-4-fluorophenol (S2). The title compound was synthesized via a similar procedure described above for the preparation of 2-cinnamylphenol (S1), except using 4-fluorophenol (2.9 $\mathrm{g}, 17.86 \mathrm{mmol}, 1.0$ equiv), cinnamyl chloride (2.5 mL, $17.86 \mathrm{mmol}, 1.0$ equiv), and sodium hydride (1.428 g, $35.7 \mathrm{mmol}, 2.0$ equiv). The crude material was purified via column chromatography on silica gel using 95:5 -> 90:10 hexanes:ethyl acetate as the eluent. This procedure afforded $2.617 \mathrm{~g}(64 \%)$ of the title compound as a yellow solid, mp $51-52{ }^{\circ} \mathrm{C} .{ }^{1} \mathrm{H}$ NMR $\left(500 \mathrm{MHz}^{\mathrm{CDCl}}{ }_{3}\right) \delta 7.41-7.16(\mathrm{~m}, 5 \mathrm{H}), 6.90(\mathrm{~d}, J=9.0 \mathrm{~Hz}, 1 \mathrm{H}), 6.83(\mathrm{~d}, J=13.6 \mathrm{~Hz}, 1 \mathrm{H}), 6.75$ (d, $J=13.4 \mathrm{~Hz}, 1 \mathrm{H}), 6.51(\mathrm{~d}, J=15.9 \mathrm{~Hz}, 1 \mathrm{H}), 6.35(\mathrm{~d}, J=15.9 \mathrm{~Hz}, 1 \mathrm{H}), 4.78(\mathrm{~s}, 1 \mathrm{H}), 3.54(\mathrm{~d}, J$ $=6.6 \mathrm{~Hz}, 2 \mathrm{H}) .{ }^{13} \mathrm{C}$ NMR $\left(126 \mathrm{MHz}, \mathrm{CDCl}_{3}\right) \delta 158.1,156.2,149.8(\mathrm{~d}, \mathrm{~J}=2.5 \mathrm{~Hz}), 136.9,132.0$, 128.6, $127.4(\mathrm{~d}, J=7.5 \mathrm{~Hz}), 127.0,126.2,116.6(\mathrm{~d}, J=31 \mathrm{~Hz}), 116.5(\mathrm{~d}, J=18 \mathrm{~Hz}), 113.9(\mathrm{~d}, J$ $=23 \mathrm{~Hz}$ ), 34.0. IR (film) 3411.7, $1619.3 \mathrm{~cm}^{-1}$. HRMS (ESl- TOF) m/z: $\left[M-\mathrm{H}^{+}\right]^{-}$calcd for $\mathrm{C}_{15} \mathrm{H}_{13} \mathrm{FO} 227.0872$; found 227.0878. 


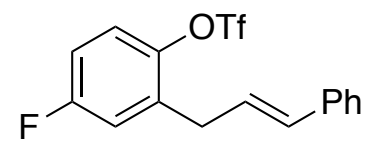

2-Cinnamyl-4-fluorophenyl trifluoromethanesulfonate (4f). The title compound was synthesized via a similar procedure described above for the preparation of 2-cinnamylphenyl trifluoromethanesulfonate (4e), except using 2-cinnamyl-4-fluorophenol (S2) $(0.79 \mathrm{~g}, 3.47 \mathrm{mmol}$, 1.0 equiv), trifluoromethanesulfonic anhydride $(1.16 \mathrm{~mL}, 6.94 \mathrm{mmol}, 2.0$ equiv), and pyridine (0.56 mL, $6.94 \mathrm{mmol}, 2.0$ equiv). The crude material was purified via column chromatography on silica gel using 100:1 -> 98:2 hexanes:ethyl acetate as the eluent. This procedure afforded $0.8474 \mathrm{~g}(68 \%)$ of the title compound as a colorless oil. ${ }^{1} \mathrm{H} \mathrm{NMR}\left(500 \mathrm{MHz}, \mathrm{CDCl}_{3}\right) \delta 7.52-$ $7.22(\mathrm{~m}, 6 \mathrm{H}), 7.15(\mathrm{~d}, J=8.7 \mathrm{~Hz}, 1 \mathrm{H}), 7.04(\mathrm{~s}, 1 \mathrm{H}), 6.60(\mathrm{~d}, J=15.8 \mathrm{~Hz}, 1 \mathrm{H}), 6.31$ (dd, $J=15.8$, $7.0 \mathrm{~Hz}, 1 \mathrm{H}), 3.69(\mathrm{~d}, J=7.0 \mathrm{~Hz}, 2 \mathrm{H}) .{ }^{13} \mathrm{C} \mathrm{NMR}\left(126 \mathrm{MHz}, \mathrm{CDCl}_{3}\right) \delta 162.6,160.6,143.5(\mathrm{~d}, J=4$ $\mathrm{Hz}), 136.8,135.9$ (d, $J=8 \mathrm{~Hz}), 133.5,127.7,126.3,125.1,123.1$ (d, $J=9 \mathrm{~Hz}), 118.7$ (q, $J=$ $319 \mathrm{~Hz}), 117.9$ (d, $J=24 \mathrm{~Hz}), 115.0$ (d, $J=24 \mathrm{~Hz})$, 33.3. IR (film) 1592.3 $\mathrm{cm}^{-1}$. HRMS (ESI ${ }^{+}$ TOF) $\mathrm{m} / \mathrm{z}:\left[\mathrm{M}+\mathrm{H}^{+}\right]^{+}$calcd for $\mathrm{C}_{16} \mathrm{H}_{12} \mathrm{~F}_{4} \mathrm{O}_{3} \mathrm{~S} 361.0522$; found 361.0516 .

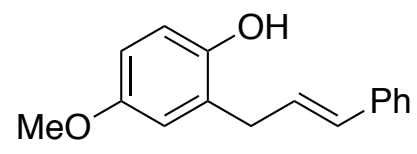

2-Cinnamyl-4-methoxyphenol (S3). The title compound was synthesized via a similar procedure described above for the preparation of 2-cinnamylphenol (S1), except using 4methoxyphenol (1.9 g, $14.4 \mathrm{mmol}, 1.0$ equiv), cinnamyl chloride (2.0 mL, $14.4 \mathrm{mmol}, 1.0$ equiv), and sodium hydride ( $1.15 \mathrm{~g}, 28.8 \mathrm{mmol}, 2.0$ equiv). The crude material was purified via column chromatography on silica gel using 95:5 -> 90:10 hexanes:ethyl acetate as the eluent. This procedure afforded $1.516 \mathrm{~g}(44 \%)$ of the title compound as an orange solid, $\mathrm{mp} 74-76{ }^{\circ} \mathrm{C} .{ }^{1} \mathrm{H}$ NMR $\left(500 \mathrm{MHz}, \mathrm{CDCl}_{3}\right) \delta 7.46-7.10(\mathrm{~m}, 5 \mathrm{H}), 6.83-6.61(\mathrm{~m}, 3 \mathrm{H}), 6.50(\mathrm{~d}, J=15.9 \mathrm{~Hz}, 1 \mathrm{H})$, $6.37(\mathrm{~d}, J=15.8 \mathrm{~Hz}, 1 \mathrm{H}), 4.59(\mathrm{~s}, 1 \mathrm{H}), 3.76(\mathrm{~s}, 3 \mathrm{H}), 3.54(\mathrm{~d}, J=6.3 \mathrm{~Hz}, 2 \mathrm{H}) .{ }^{13} \mathrm{C}$ NMR $(126$ 
$\left.\mathrm{MHz}, \mathrm{CDCl}_{3}\right) \delta 153.8,137.0,131.6,128.6$ (2 peaks), 128.5, 127.7, 126.8, 126.2, 116.5, 116.0, 112.6, 55.7, 34.3. IR (film) 3384.8, 1598.5, $\mathrm{cm}^{-1}$. HRMS (ESI- TOF) $\mathrm{m} / \mathrm{z}$ : $\left[\mathrm{M}-\mathrm{H}^{+}\right]^{-}$calcd for $\mathrm{C}_{16} \mathrm{H}_{16} \mathrm{O}_{2} 239.1072$; found 239.1078.<smiles>COc1ccc(OC)c(C/C=C/c2ccccc2)c1</smiles>

2-Cinnamyl-4-methoxyphenyl trifluoromethanesulfonate $(\mathbf{4 g})$. The title compound was synthesized via a similar procedure described above for the preparation of 2-cinnamylphenyl trifluoromethanesulfonate (4e), except using 2-cinnamyl-4-methoxyphenol (S3) (1.0 g, 4.17 mmol, 1.0 equiv), trifluoromethanesulfonic anhydride (1.4 mL, $8.33 \mathrm{mmol}, 2.0$ equiv), and pyridine $(0.67 \mathrm{~mL}, 8.33 \mathrm{mmol}, 2.0$ equiv). The crude material was purified via column chromatography on silica gel using 98:2 -> 95:5 hexanes:ethyl acetate as the eluent. This procedure afforded $1.18 \mathrm{~g}(76 \%)$ of the title compound as a colorless oil. ${ }^{1} \mathrm{H}$ NMR $(500 \mathrm{MHz}$, $\left.\mathrm{CDCl}_{3}\right) \delta 7.44-7.13(\mathrm{~m}, 6 \mathrm{H}), 6.87(\mathrm{~s}, 1 \mathrm{H}), 6.79(\mathrm{~d}, J=9.0 \mathrm{~Hz}, 1 \mathrm{H}), 6.51(\mathrm{~d}, J=15.8 \mathrm{~Hz}, 1 \mathrm{H})$, $6.34-6.18(\mathrm{~m}, 1 \mathrm{H}), 3.80(\mathrm{~s}, 3 \mathrm{H}), 3.60(\mathrm{~d}, J=6.9 \mathrm{~Hz}, 2 \mathrm{H}) .{ }^{13} \mathrm{C} \mathrm{NMR}\left(126 \mathrm{MHz}, \mathrm{CDCl}_{3}\right) \delta$ 159.0, 141.3, 137.0, 134.4, 132.8, 128.5, 127.4, 126.2, 126.0, 122.4, 118.7 (q, Jc-F $=305 \mathrm{~Hz}$ ), 116.3, 112.8, 55.7, 33.5. IR (film) $1587.1 \mathrm{~cm}^{-1}$. HRMS (ESI ${ }^{+}$TOF) $\mathrm{m} / \mathrm{z}:\left[\mathrm{M}+\mathrm{NH}_{4}^{+}\right]^{+}$calcd for $\mathrm{C}_{17} \mathrm{H}_{15} \mathrm{~F}_{3} \mathrm{O}_{4} \mathrm{~S} 390.0987$; found 390.0981 .

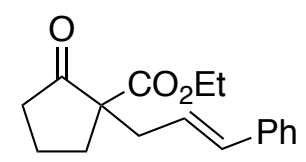

Ethyl 1-cinnamyl-2-oxocyclopentane-1-carboxylate (S4). A flame-dried flask equipped with a stir bar was cooled under a stream of nitrogen and charged with sodium hydride $(60 \%$ in mineral oil, $0.534 \mathrm{~g}, 13.35 \mathrm{mmol}, 0.89$ equiv) and THF (6 mL). The mixture was cooled to $0{ }^{\circ} \mathrm{C}$ and a solution of ethyl-2-oxocyclopentanecarboxylate $(1.9 \mathrm{~mL}, 15 \mathrm{mmol}, 1$ equiv) in THF (10 mL) was added dropwise over the course of $90 \mathrm{~min}$. The reaction mixture was warmed to rt and stirred 
for $90 \mathrm{~min}$, then a solution of cinnamyl bromide (2.6 mL, $17.25 \mathrm{mmol}, 1.15$ equiv) in THF (5 mL) was added and the mixture was stirred at $\mathrm{rt}$ for $16 \mathrm{~h}$. The reaction was quenched with saturated aqueous ammonium chloride $(20 \mathrm{~mL})$ and transferred to a separatory funnel. The layers were separated and the aqueous layer was extracted with ethyl acetate $(3 \times 20 \mathrm{~mL})$. The combined organic layers were washed with brine $(1 \times 20 \mathrm{~mL})$ and then dried over $\mathrm{Na}_{2} \mathrm{SO}_{4}$, filtered and concentrated in vacuo to afford a yellow oil. The crude material was purified via column chromatography on silica gel using 90:10 hexanes:ethyl acetate as the eluent. This procedure afforded $1.84 \mathrm{~g}(45 \%)$ of the title compound as a colorless oil. ${ }^{1} \mathrm{H}$ NMR $\left(500 \mathrm{MHz}, \mathrm{CDCl}_{3}\right) \delta 7.40$ - $7.25(\mathrm{~m}, 4 \mathrm{H}), 7.21(\mathrm{~d}, J=14.2 \mathrm{~Hz}, 1 \mathrm{H}), 6.45(\mathrm{~d}, J=15.8 \mathrm{~Hz}, 1 \mathrm{H}), 6.16-6.01(\mathrm{~m}, 1 \mathrm{H}), 4.25-$ $4.10(m, 2 H), 2.89-2.73(m, 1 H), 2.59-2.37(m, 3 H), 2.31-2.16(m, 1 H), 2.04(d, J=14.0$ $\mathrm{Hz}, 2 \mathrm{H}), 1.90(\mathrm{~d}, J=17.0 \mathrm{~Hz}, 1 \mathrm{H}), 1.25(\mathrm{t}, J=7.1 \mathrm{~Hz}, 3 \mathrm{H}) .{ }^{13} \mathrm{C} \mathrm{NMR}\left(126 \mathrm{MHz}, \mathrm{CDCl}_{3}\right) \delta 214.6$, $170.9,137.0,134.1,128.5,127.4,126.2,124.5,61.5,60.2,38.1,37.0,32.3,19.6,14.1$. IR (film) 1750.4, 1719.9, $1448.4 \mathrm{~cm}^{-1}$. HRMS (ESI ${ }^{+}$TOF) $\mathrm{m} / \mathrm{z}:\left[\mathrm{M}+\mathrm{H}^{+}\right]^{+}$calcd for $\mathrm{C}_{17} \mathrm{H}_{20} \mathrm{O}_{3} 272.1413$; found 273.1485 .

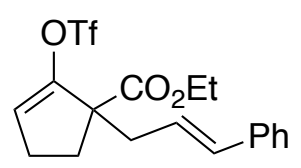

Ethyl 1-cinnamyl-2-\{[(trifluoromethyl)sulfonyl]oxy\}cyclopent-2-ene-1-carboxylate (1j). A flame-dried flask equipped with a stir bar was cooled under a stream of nitrogen and charged with THF (10 mL) and diisopropylamine $(1.0 \mathrm{~mL}, 7.29 \mathrm{mmol}, 1.7$ equiv). The mixture was cooled to $0{ }^{\circ} \mathrm{C}$, n-BuLi (2.5 $\mathrm{M}$ in hexanes, $2.9 \mathrm{~mL}, 7.29 \mathrm{mmol}, 1.7$ equiv) was added dropwise, and the resulting mixture was stirred at $0{ }^{\circ} \mathrm{C}$ for $30 \mathrm{~min}$. The mixture was cooled to $-78{ }^{\circ} \mathrm{C}$ and a solution of ethyl 1-cinnamyl-2-oxocyclopentane-1-carboxylate (S4, $1.17 \mathrm{~g}, 4.29 \mathrm{mmol}, 1$ equiv) in THF $(10 \mathrm{~mL})$ was added dropwise over the course of $90 \mathrm{~min}$. The reaction mixture was stirred at -78 ${ }^{\circ} \mathrm{C}$ for $2.5 \mathrm{~h}$, then $\mathrm{N}$-(2-pyridyl)triflamide $(2.61 \mathrm{~g}, 7.29 \mathrm{mmol}, 1.7$ equiv) in THF $(5 \mathrm{~mL})$ was added, the mixture was warmed to rt and stirred for $16 \mathrm{~h}$. The reaction was quenched with water 
$(20 \mathrm{~mL})$ and the mixture was transferred to a separatory funnel. The layers were separated and the aqueous layer was extracted with ethyl acetate $(3 \times 20 \mathrm{~mL})$. The combined organic layers were washed with brine $(1 \times 20 \mathrm{~mL})$ and then dried over $\mathrm{Na}_{2} \mathrm{SO}_{4}$, filtered and concentrated in vacuo to afford a yellow oil. The crude material was purified via column chromatography on silica gel using 98:2 -> 95:5 hexanes:ethyl acetate as the eluent. This procedure afforded $1.18 \mathrm{~g}$ $(68 \%)$ of the title compound as a yellow oil. ${ }^{1} \mathrm{H}$ NMR $\left(500 \mathrm{MHz}, \mathrm{CDCl}_{3}\right) \delta 7.42-7.14(\mathrm{~m}, 5 \mathrm{H})$, $6.50(\mathrm{~d}, J=15.7 \mathrm{~Hz}, 1 \mathrm{H}), 6.14-6.00(\mathrm{~m}, 1 \mathrm{H}), 5.80(\mathrm{~s}, 1 \mathrm{H}), 4.32-4.11(\mathrm{~m}, 2 \mathrm{H}), 2.78(\mathrm{~d}, J=8.0$ $\mathrm{Hz}, 1 \mathrm{H}), 2.63(\mathrm{~d}, J=14.1 \mathrm{~Hz}, 1 \mathrm{H}), 2.48(\mathrm{~d}, J=9.3 \mathrm{~Hz}, 2 \mathrm{H}), 2.35(\mathrm{~d}, J=5.5 \mathrm{~Hz}, 1 \mathrm{H}), 2.15-1.93$ $(\mathrm{m}, 1 \mathrm{H}), 1.29(\mathrm{t}, J=7.2 \mathrm{~Hz}, 3 \mathrm{H}) .{ }^{13} \mathrm{C} \operatorname{NMR}\left(126 \mathrm{MHz}, \mathrm{CDCl}_{3}\right) \delta 172.5,147.9,137.0,134.5$, 128.5, 127.5, 126.2, 123.6, $121.6\left(q, J_{C-F}=375 \mathrm{~Hz}\right), 118.3,61.6,57.6,38.0,31.2,26.2,14.0 . \mathrm{IR}$ (film) 1728.6, 1602.5, $1422.8 \mathrm{~cm}^{-1}$. HRMS (ESI ${ }^{+}$TOF) $\mathrm{m} / \mathrm{z}:\left[\mathrm{M}+\mathrm{H}^{+}\right]^{+}$calcd for $\mathrm{C}_{18} \mathrm{H}_{19} \mathrm{~F}_{3} \mathrm{O}_{5} \mathrm{~S}$ 405.0984; found 405.0978.

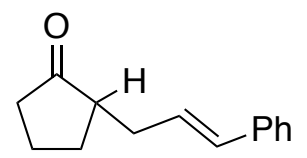

2-Cinnamylcyclopentan-1-one (S5). A flame-dried flask equipped with a stir bar was cooled under a stream of nitrogen and charged with palladium allyl chloride dimer $(0.146 \mathrm{~g}, 0.4 \mathrm{mmol}$, 0.02 equiv), dppf (0.665 g, $1.2 \mathrm{mmol}, 0.06 \mathrm{mmol})$, and methanol $(80 \mathrm{~mL})$. The mixture was stirred at rt for $60 \mathrm{~min}$, then cinnamyl alcohol $(2.8 \mathrm{~mL}, 22 \mathrm{mmol}, 1.1$ equiv) was added to the orange mixture, and the resulting mixture was stirred at rt for $30 \mathrm{~min}$. Cyclopentanone $(2.1 \mathrm{~mL}$, $20 \mathrm{mmol}, 1$ equiv) and pyrrolidine $(0.33 \mathrm{~mL}, 4 \mathrm{mmol}, 0.2 \mathrm{mmol})$ were added, and the mixture was then heated to $45^{\circ} \mathrm{C}$ with stirring for $16 \mathrm{~h}$. The reaction was quenched with cold saturated aqueous ammonium chloride $(20 \mathrm{~mL})$. The mixture was transferred to a separatory funnel, the layers were separated, and the aqueous layer was extracted with diethyl ether $(3 \times 20 \mathrm{~mL})$. The combined organic layers were washed with brine $(1 \times 20 \mathrm{~mL})$ and then dried over $\mathrm{Na}_{2} \mathrm{SO}_{4}$, filtered, and concentrated in vacuo to afford yellow oil. The crude material was purified via 
column chromatography on silica gel using 98:2 -> 95:5 hexanes:ethyl acetate as the eluent. This procedure afforded $3.379 \mathrm{~g}(83 \%)$ of the title compound as a colorless oil. ${ }^{1} \mathrm{H}$ NMR $(500$ $\left.\mathrm{MHz}, \mathrm{CDCl}_{3}\right) \delta 7.39-7.13(\mathrm{~m}, 5 \mathrm{H}), 6.43(\mathrm{~d}, J=15.6 \mathrm{~Hz}, 1 \mathrm{H}), 6.24-6.08(\mathrm{~m}, 1 \mathrm{H}), 2.65(\mathrm{~d}, J=$ $10.0 \mathrm{~Hz}, 1 \mathrm{H}), 2.41-2.16(\mathrm{~m}, 4 \mathrm{H}), 2.11(\mathrm{~d}, \mathrm{~J}=8.8 \mathrm{~Hz}, 1 \mathrm{H}), 2.01(\mathrm{~s}, 1 \mathrm{H}), 1.81$ (dddt, $J=14.7$, 8.5, 4.3, $2.3 \mathrm{~Hz}, 1 \mathrm{H}), 1.63(\mathrm{~s}, 1 \mathrm{H}) .{ }^{13} \mathrm{C} \operatorname{NMR}\left(126 \mathrm{MHz}, \mathrm{CDCl}_{3}\right) \delta 220.4,137.4,131.8,128.5$, 127.6, 127.1, 126.0, 49.0, 38.2, 33.1, 29.0, 20.7. IR (film) 1732.7, $1597.2 \mathrm{~cm}^{-1}$. HRMS (ESI TOF) $\mathrm{m} / \mathrm{z}:\left[\mathrm{M}+\mathrm{H}^{+}\right]^{+}$calcd for $\mathrm{C}_{14} \mathrm{H}_{16} \mathrm{O} 201.1280$; found 201.1274.

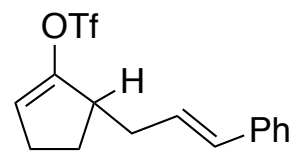

5-Cinnamylcyclopent-1-en-1-yl trifluoromethanesulfonate (1k). A flame-dried flask equipped with a stir bar was cooled under a stream of nitrogen and charged with THF $(20 \mathrm{~mL})$ and diisopropylamine $\left(1.05 \mathrm{~mL}, 7.5 \mathrm{mmol}, 1.5\right.$ equiv). The mixture was cooled to $0{ }^{\circ} \mathrm{C}, n$-BuLi $(2.5 \mathrm{M}$ in hexanes, $3.0 \mathrm{~mL}, 7.5 \mathrm{mmol}, 1.5$ equiv) was added dropwise, and the resulting solution was stirred at $0{ }^{\circ} \mathrm{C}$ for $30 \mathrm{~min}$. The mixture was then cooled to $-78{ }^{\circ} \mathrm{C}$ and a solution of 2cinnamylcyclopentan-1-one (S5, $1.0 \mathrm{~g}, 5.0 \mathrm{mmol}, 1$ equiv) in THF (20 mL) was added dropwise over the course of $70 \mathrm{~min}$. The reaction mixture was then stirred for $2 \mathrm{~h}$ at $-78{ }^{\circ} \mathrm{C}$, then a solution of $\mathrm{N}$-(2-pyridyl)triflamide $(2.61 \mathrm{~g}, 7.29 \mathrm{mmol}, 1.2$ equiv) in THF (10 mL) was added. The resulting mixture was warmed to $-41^{\circ} \mathrm{C}$ in $\mathrm{CH}_{3} \mathrm{CN} / \mathrm{dry}$ ice bath and stirred for $2 \mathrm{~h}$. The reaction was quenched with water $(20 \mathrm{~mL})$ and the mixture was transferred to a separatory funnel. The layers were separated and the aqueous layer was extracted with ethyl acetate $(3 \times 20 \mathrm{~mL})$. The combined organic layers were washed with brine $(1 \times 20 \mathrm{~mL})$ and then dried over $\mathrm{Na}_{2} \mathrm{SO}_{4}$, filtered, and concentrated in vacuo to afford a yellow oil. The crude material was purified via column chromatography on silica gel using 100:0 -> 98:2 hexanes:ethyl acetate as the eluent. This procedure afforded $0.8675 \mathrm{~g}(52 \%)$ of the title compound as a colorless oil. ${ }^{1} \mathrm{H}$ NMR $(500$ $\left.\mathrm{MHz}_{\mathrm{CDCl}}\right) \delta 7.35(\mathrm{~d}, J=18.6 \mathrm{~Hz}, 4 \mathrm{H}), 7.26(\mathrm{~s}, 1 \mathrm{H}), 6.49(\mathrm{~d}, J=15.8 \mathrm{~Hz}, 1 \mathrm{H}), 6.18(\mathrm{~d}, J=$ 
$15.7 \mathrm{~Hz}, 1 \mathrm{H}), 5.71(\mathrm{~s}, 1 \mathrm{H}), 3.04(\mathrm{~s}, 1 \mathrm{H}), 2.57$ (ddt, $J=11.1,5.8,2.9 \mathrm{~Hz}, 1 \mathrm{H}), 2.45-2.26(\mathrm{~m}$, 3H), $2.20(\mathrm{~s}, 1 \mathrm{H}), 1.80(\mathrm{~d}, J=6.2 \mathrm{~Hz}, 1 \mathrm{H}) .{ }^{13} \mathrm{C} \mathrm{NMR}\left(126 \mathrm{MHz}, \mathrm{CDCl}_{3}\right) \delta 151.3,137.3,132.6$, 128.5, 127.2, 126.3, 126.1, $121.1\left(q, J_{C-F}=315 \mathrm{~Hz}\right), 117.4,43.0,35.7,26.7,26.6$. IR (film) 1656.6, $1495.7 \mathrm{~cm}^{-1}$. HRMS (ESI TOF) $\mathrm{m} / \mathrm{z}$ : $\left[\mathrm{M}+\mathrm{H}^{+}\right]^{+}$calcd for $\mathrm{C}_{15} \mathrm{H}_{15} \mathrm{~F}_{3} \mathrm{O}_{3} \mathrm{~S}$ 333.0772; found 333.0767.

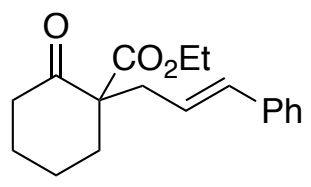

Ethyl 1-cinnamyl-2-oxocyclohexane-1-carboxylate (S6). The title compound was synthesized via a similar procedure described above for the preparation of ethyl 1-cinnamyl-2oxocyclopentane-1-carboxylate (S4), except using ethyl-2-oxocyclohexanecarboxylate (3.2 mL, $20 \mathrm{mmol}, 1$ equiv), cinnamyl bromide (3.4 mL, $23.0 \mathrm{mmol}, 1.15$ equiv), and sodium hydride (0.712 g, $17.8 \mathrm{mmol}, 0.89$ equiv). The crude material was purified via column chromatography on silica gel using 95:5 -> 90:10 hexanes:ethyl acetate as the eluent. This procedure afforded $3.58 \mathrm{~g}(63 \%)$ of the title compound as a yellow oil. ${ }^{1} \mathrm{H}$ NMR $\left(500 \mathrm{MHz}, \mathrm{CDCl}_{3}\right) \delta 7.39-7.12(\mathrm{~m}$, $5 \mathrm{H}), 6.37(\mathrm{~d}, J=15.8 \mathrm{~Hz}, 1 \mathrm{H}), 6.24-6.09(\mathrm{~m}, 1 \mathrm{H}), 4.16(\mathrm{q}, J=7.1 \mathrm{~Hz}, 2 \mathrm{H}), 2.73$ (tdd, $J=11.5$, 7.1, $1.4 \mathrm{~Hz}, 1 \mathrm{H}), 2.56-2.41(\mathrm{~m}, 3 \mathrm{H}), 2.01(\mathrm{ddt}, J=9.2,6.2,3.1 \mathrm{~Hz}, 1 \mathrm{H}), 1.81-1.57(\mathrm{~m}, 4 \mathrm{H})$, $1.57-1.49(\mathrm{~m}, 1 \mathrm{H}), 1.21(\mathrm{t}, J=7.1 \mathrm{~Hz}, 3 \mathrm{H}) .{ }^{13} \mathrm{C}$ NMR $\left(126 \mathrm{MHz}, \mathrm{CDCl}_{3}\right) \delta 207.6,171.5,137.2$ 133.2, 128.4, 127.2, 126.1, 125.1, 61.3, 41.2, 38.6, 36.1, 27.9, 27.5, 22.5, 14.2. IR (film) 1709.8 (2 overlapping peaks), 1597.9, 1495.7, $\mathrm{cm}^{-1}$. HRMS $\left(E S I^{+}\right.$TOF) $\mathrm{m} / \mathrm{z}$ : $\left[\mathrm{M}+\mathrm{H}^{+}\right]^{+}$calcd for $\mathrm{C}_{18} \mathrm{H}_{22} \mathrm{O}_{3} 287.1647$; found 287.1642.<smiles>CCOC1=CCCCC1(C/C=C/c1ccccc1)OCC</smiles> 
Ethyl 1-cinnamyl-2-\{[(trifluoromethyl)sulfonyl]oxy\}cyclohex-2-ene-1-carboxylate (1g). The title compound was synthesized a similar procedure described above for the preparation of ethyl 1-cinnamyl-2-\{(trifluoromethyl)sulfonyl]oxy\}cyclopent-2-ene-1-carboxylate (1j), except using ethyl 1-cinnamyl-2-oxocyclohexane-1-carboxylate (S6) (1.13 g, 4.0 mmol, 1 equiv), LDA (6.73 mmol, 1.7 equiv), and $\mathrm{N}$-(2-pyridyl)triflamide $(2.409 \mathrm{~g}, 6.73 \mathrm{mmol}, 1.7$ equiv). The crude material was purified via column chromatography on silica gel using 100:0 -> 98:2 hexanes:ethyl acetate as the eluent. This procedure afforded $1.06 \mathrm{~g}(64 \%)$ of the title compound as a colorless oil. ${ }^{1} \mathrm{H}$ $\operatorname{NMR}\left(500 \mathrm{MHz}, \mathrm{CDCl}_{3}\right) \delta 7.46-7.12(\mathrm{~m}, 5 \mathrm{H}), 6.49(\mathrm{~d}, J=15.7 \mathrm{~Hz}, 1 \mathrm{H}), 6.12(\mathrm{t}, J=15.4 \mathrm{~Hz}$ $1 \mathrm{H}), 5.92(\mathrm{~s}, 1 \mathrm{H}), 4.36-4.11(\mathrm{~m}, 2 \mathrm{H}), 2.82-2.64(\mathrm{~m}, 2 \mathrm{H}), 2.38-2.10(\mathrm{~m}, 3 \mathrm{H}), 1.82-1.53(\mathrm{~m}$, $3 \mathrm{H}), 1.31(\mathrm{t}, J=7.1 \mathrm{~Hz}, 3 \mathrm{H}) .{ }^{13} \mathrm{C}$ NMR $\left(126 \mathrm{MHz}, \mathrm{CDCl}_{3}\right) \delta 172.6,148.1,137.0,134.5,128.5$, 127.5, 126.2, 123.8, 120.3, 118.3 (q, JC-F $=318 \mathrm{~Hz}$ ), 61.8, 50.5, 38.7, 32.3, 24.4, 18.6, 14.0. IR (film) 1730.3, $1677.1 \mathrm{~cm}^{-1}$. HRMS (ESI TOF) $\mathrm{m} / \mathrm{z}:\left[\mathrm{M}+\mathrm{H}^{+}\right]^{+}$calcd for $\mathrm{C}_{19} \mathrm{H}_{21} \mathrm{~F}_{3} \mathrm{O}_{5} \mathrm{~S}$ 419.1140; found 419.1135 .

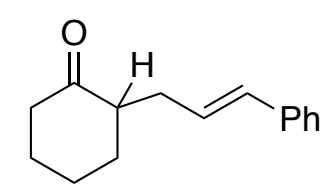

2-Cinnamylcyclohexan-1-one (S7). The title compound was synthesized via a similar procedure described above for the preparation of 2-cinnamylcyclopentan-1-one (S5), except using cyclohexanone $(2.1 \mathrm{~mL}, 20 \mathrm{mmol}, 1$ equiv), cinnamyl alcohol $(2.8 \mathrm{~mL}, 2.2 \mathrm{mmol}, 1.1$ equiv), palladium allyl chloride dimer ( $0.146 \mathrm{~g}, 0.4 \mathrm{mmol}, 0.02$ equiv), dppf (0.665 g, $1.2 \mathrm{mmol}$, $0.06 \mathrm{mmol})$, and pyrrolidine $(0.33 \mathrm{~mL}, 4.0 \mathrm{mmol}, 0.2$ equiv). The crude material was purified via column chromatography on silica gel using 95:5 hexanes:ethyl acetate as the eluent. This procedure afforded $2.74 \mathrm{~g} \mathrm{(63 \% )}$ of the title compound as a yellow oil. ${ }^{1} \mathrm{H}$ NMR $(500 \mathrm{MHz}$, $\left.\mathrm{CDCl}_{3}\right) \delta 7.44-7.23(\mathrm{~m}, 4 \mathrm{H}), 7.19(\mathrm{~s}, 1 \mathrm{H}), 6.39(\mathrm{~d}, J=15.8 \mathrm{~Hz}, 1 \mathrm{H}), 6.20(\mathrm{t}, J=15.2 \mathrm{~Hz}, 1 \mathrm{H})$, $2.73-2.60(\mathrm{~m}, 1 \mathrm{H}), 2.43(\mathrm{~d}, J=15.8 \mathrm{~Hz}, 2 \mathrm{H}), 2.32(\mathrm{~d}, J=19.2 \mathrm{~Hz}, 1 \mathrm{H}), 2.25-2.00(\mathrm{~m}, 3 \mathrm{H})$, 
$1.88(\mathrm{~s}, 1 \mathrm{H}), 1.67(\mathrm{~s}, 2 \mathrm{H}), 1.41(\mathrm{~d}, \mathrm{~J}=12.4 \mathrm{~Hz}, 1 \mathrm{H}) .{ }^{13} \mathrm{C} \mathrm{NMR}\left(126 \mathrm{MHz}, \mathrm{CDCl}_{3}\right) \delta 212.4,137.5$, 131.6, 128.5, 128.4, 128.3, 127.0, 126.0, 50.7, 42.1, 33.6, 27.9, 25.0. IR (film) 1705.24, 1597.9 $\mathrm{cm}^{-1}$. HRMS (ESI ${ }^{+}$TOF) m/z: $\left[\mathrm{M}+\mathrm{H}^{+}\right]^{+}$calcd for $\mathrm{C}_{15} \mathrm{H}_{18} \mathrm{O} 215.1436$; found 215.1430.

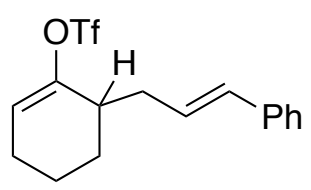

6-Cinnamylcyclohex-1-en-1-yl trifluoromethanesulfonate (1h). The title compound was synthesized via a similar procedure to 5-cinnamylcyclopent-1-en-1-yl trifluoromethanesulfonate (1k), except using 2-cinnamylcyclohexan-1-one (S7) (0.544 g, 2.54 mmol, 1 equiv), LDA (3.82 mmol, 1.5 equiv), and $N$-(2-pyridyl)triflamide (1.09 g, $3.05 \mathrm{mmol}, 1.2$ equiv). The crude material was purified via column chromatography on silica gel using 100:0 -> 98:2 -> 95:5 hexanes:ethyl acetate as the eluent. This procedure afforded $0.660 \mathrm{~g}(75 \%)$ of the title compound as a colorless oil. ${ }^{1} \mathrm{H}$ NMR $\left(500 \mathrm{MHz}, \mathrm{CDCl}_{3}\right) \delta 7.43-7.13(\mathrm{~m}, 5 \mathrm{H}), 6.45(\mathrm{~d}, J=15.6 \mathrm{~Hz}, 1 \mathrm{H}), 6.23-$ $6.04(\mathrm{~m}, 1 \mathrm{H}), 5.83(\mathrm{~s}, 1 \mathrm{H}), 2.62(\mathrm{~d}, J=10.1 \mathrm{~Hz}, 2 \mathrm{H}), 2.40-2.26(\mathrm{~m}, 1 \mathrm{H}), 2.18(\mathrm{~s}, 2 \mathrm{H}), 1.88(\mathrm{~s}$, 1H), 1.62 (dd, $J=49.8,6.2 \mathrm{~Hz}, 3 \mathrm{H}) .{ }^{13} \mathrm{C} \mathrm{NMR}\left(126 \mathrm{MHz}, \mathrm{CDCl}_{3}\right) \delta 151.7,137.3,132.7,128.5$, 127.2, 126.7, 126.1, 119.5, $118.5\left(q, J_{C-F}=319 \mathrm{~Hz}\right), 37.6,35.1,27.8,24.3,19.0$. IR (film) 1680.2, $1494.3 \mathrm{~cm}^{-1}$. HRMS (ESI ${ }^{+}$TOF) $\mathrm{m} / \mathrm{z}:\left[\mathrm{M}+\mathrm{NH}_{4}^{+}\right]^{+}$calcd for $\mathrm{C}_{16} \mathrm{H}_{17} \mathrm{~F}_{3} \mathrm{O}_{3} \mathrm{~S}$ 364.1194; found 364.1189.

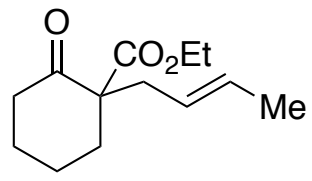

Ethyl 1-cinnamyl-2-oxocyclohexane-1-carboxylate (S8). The title compound was synthesized via a similar procedure described above for the preparation of ethyl 1-cinnamyl-2oxocyclopentane-1-carboxylate (S4), except using ethyl-2-oxocyclohexanecarboxylate (3.4 mL, $21.1 \mathrm{mmol}, 1$ equiv), crotyl bromide $(2.5 \mathrm{~mL}, 24.3 \mathrm{mmol}, 1.15$ equiv), and sodium hydride (0.75 
g, $18.8 \mathrm{mmol}, 0.89$ equiv). The crude material was purified via column chromatography on silica gel using 98:2 hexanes:ethyl acetate as the eluent. This procedure afforded $2.26 \mathrm{~g}(48 \%)$ of the title compound as a brown oil. ${ }^{1} \mathrm{H}$ NMR $\left(500 \mathrm{MHz}, \mathrm{CDCl}_{3}\right) \delta 5.54-5.22(\mathrm{~m}, 2 \mathrm{H}), 4.26-4.04(\mathrm{~m}$, $2 \mathrm{H}), 2.60-2.28(\mathrm{~m}, 4 \mathrm{H}), 2.28-2.13(\mathrm{~m}, 1 \mathrm{H}), 1.96(\mathrm{ddd}, J=14.1,5.2,2.9 \mathrm{~Hz}, 1 \mathrm{H}), 1.81-1.51$ $(\mathrm{m}, 6 \mathrm{H}), 1.42(\mathrm{~d}, J=16.2 \mathrm{~Hz}, 1 \mathrm{H}), 1.21(\mathrm{t}, J=7.1 \mathrm{~Hz}, 3 \mathrm{H}) .{ }^{13} \mathrm{C}$ NMR $\left(126 \mathrm{MHz}, \mathrm{CDCl}_{3}\right) \delta 207.7$, 171.6, 128.8, 125.6, 61.1, 41.1, 38.0, 35.7, 27.5, 22.5, 22.4, 17.9, 14.1. IR (film) 1710.4 (2 overlapping peaks), $1438.0 \mathrm{~cm}^{-1}$. HRMS (ESI+ TOF) $\mathrm{m} / \mathrm{z}$ : $\left[\mathrm{M}+\mathrm{H}^{+}\right]^{+}$calcd for $\mathrm{C}_{13} \mathrm{H}_{20} \mathrm{O}_{3} 225.1491$; found 225.1485 .

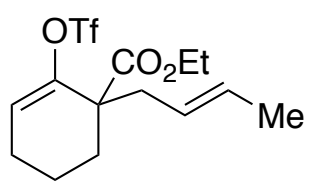

\section{(E)-Ethyl-1-(but-2-en-1-yl)-2-\{[(trifluoromethyl)sulfonyl]oxy\}cyclohex-2-ene-1-carboxylate}

(1i). The title compound was synthesized via a similar procedure described above for the preparation of ethyl 1-cinnamyl-2-\{[(trifluoromethyl)sulfonyl]oxy\}cyclohex-2-ene-1-carboxylate (1j), except using ethyl 1-cinnamyl-2-oxocyclohexane-1-carboxylate (S8) (0.98 g, 4.46 mmol, 1 equiv), LDA (7.6 mmol, 1.7 equiv), and $\mathrm{N}$-(2-pyridyl)triflamide (2.66 g, $7.6 \mathrm{mmol}, 1.7$ equiv). The crude material was purified via column chromatography on silica gel using 100:0 -> 98:2 hexanes:ethyl acetate as the eluent. This procedure afforded $0.623 \mathrm{~g}(40 \%)$ of the title compound as a colorless oil. ${ }^{1} \mathrm{H}$ NMR $\left(500 \mathrm{MHz}^{\mathrm{C}} \mathrm{CDCl}_{3}\right) \delta 5.86(\mathrm{~s}, 1 \mathrm{H}), 5.57-5.45(\mathrm{~m}, 1 \mathrm{H})$, $5.28(\mathrm{~d}, J=15.0 \mathrm{~Hz}, 1 \mathrm{H}), 4.15(\mathrm{~d}, J=16.3 \mathrm{~Hz}, 2 \mathrm{H}), 2.47(\mathrm{~s}, 2 \mathrm{H}), 2.29-2.08(\mathrm{~m}, 3 \mathrm{H}), 1.63(\mathrm{~d}, J$ $=15.5 \mathrm{~Hz}, 6 \mathrm{H}), 1.27(\mathrm{t}, J=7.1 \mathrm{~Hz}, 3 \mathrm{H}) .{ }^{13} \mathrm{C} \operatorname{NMR}\left(126 \mathrm{MHz}, \mathrm{CDCl}_{3}\right) \delta 172.8,148.2,130.2$, 124.5, 120.0, $118.3\left(q, J_{C-F}=318 \mathrm{~Hz}\right), 61.5,50.3,38.3,32.0,31.9,24.4,18.7,13.9$. IR (film) 1731.2, 1677.4, $1414.4 \mathrm{~cm}^{-1}$. HRMS (ESI ${ }^{+}$TOF) $\mathrm{m} / \mathrm{z}:\left[\mathrm{M}+\mathrm{H}^{+}\right]^{+}$calcd for $\mathrm{C}_{14} \mathrm{H}_{19} \mathrm{~F}_{3} \mathrm{O}_{5} \mathrm{~S} 357.0984$; found 357.0978 . 


\section{Preparation and Characterization of Products}

\section{General Procedure for Pd-catalyzed Alkene Dialkylation Reactions on terminal alkenes, General Procedure A.}

A flame-dried $4 \mathrm{~mL}$ vial equipped with a stir bar was cooled under a stream of nitrogen and charged with $\mathrm{Pd}(\mathrm{OAc})_{2}(0.008 \mathrm{mmol}, 0.04$ equiv), Brettphos $(0.012 \mathrm{mmol}, 0.06$ equiv), and lithium tert-butoxide ( $0.28 \mathrm{mmol}, 1.4$ equiv). The aryl or alkenyl triflate ( $0.2 \mathrm{mmol}, 1.0$ equiv) was weighed in a separate dram vial and diluted with toluene $(1 \mathrm{~mL}, 0.2 \mathrm{M})$. This mixture was added to the reaction vessel and the appropriate nucleophile $(0.24 \mathrm{mmol}, 1.2$ equiv) was added. Toluene $(1 \mathrm{~mL}, 0.2 \mathrm{M})$ was used to rinse the dram vial and the solution was transferred to the reaction vessel. The vial was flushed with nitrogen, capped, and heated to $95{ }^{\circ} \mathrm{C}$ with stirring overnight until the starting material had been consumed. The mixture was then cooled to $\mathrm{rt}$, quenched with saturated aqueous ammonium chloride $(1 \mathrm{~mL})$. The aqueous layer was extracted with ethyl acetate $(3 \times 1 \mathrm{~mL})$, dried over $\mathrm{MgSO}_{4}$, filtered and concentrated in vacuo. The crude product was purified via flash chromatography on silica gel to afford the desired product.

\section{General procedure for Pd-catalyzed alkene dialkylation reactions on internal alkene substrates, General procedure B}

A flame-dried $4 \mathrm{~mL}$ vial equipped with a stir bar and was cooled under a stream of nitrogen and charged with the appropriate triflate $(0.2 \mathrm{mmol}, 1.0$ equiv), the appropriate palladium precatalyst $(0.008 \mathrm{mmol}, 0.04$ equiv), the appropriate ligand $(0.012 \mathrm{mmol}, 0.06$ equiv), lithium tertbutoxide ( $0.44 \mathrm{mmol}, 2.2$ equiv). The vial was purged with nitrogen and charged with toluene $(0.8 \mathrm{M})$ and the appropriate malonate derivative $(0.6 \mathrm{mmol}, 3.6$ equiv). The vial was capped and heated to the appropriate temperature with stirring until the starting material had been consumed. The mixture was cooled to rt, charged with phenanthrene (1 equiv; NMR internal standard), diluted with dichloromethane $(1 \mathrm{~mL})$, and quenched with saturated ammonium 
chloride $(1 \mathrm{~mL})$. The aqueous layer was extracted with dichloromethane $(3 \times 1 \mathrm{~mL})$, dried over $\mathrm{Na}_{2} \mathrm{SO}_{4}$, filtered, and concentrated in vacuo. The crude product was purified via flash chromatography on silica gel to afford the desired product.

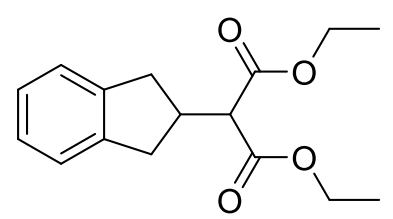

Diethyl 2-(2,3-dihydro-1H-inden-2-yl)malonate (5a). The title compound was prepared from 2allylphenyl triflate $(53.5 \mathrm{mg}, 0.2 \mathrm{mmol})$ and diethyl malonate $(37 \mu \mathrm{L}, 0.24 \mathrm{mmol})$ using General Procedure A. The crude material was purified via column chromatography on silica gel using 95:5 hexanes:ethyl acetate as the eluent. This procedure afforded $51.7 \mathrm{mg}(93 \%)$ of the title compound as a white solid $\left(\mathrm{mp} 33-35^{\circ} \mathrm{C}\right) .{ }^{1} \mathrm{H}$ NMR $\left(500 \mathrm{MHz}, \mathrm{CDCl}_{3}\right) \delta 7.22-7.15(\mathrm{~m}, 2 \mathrm{H})$, $7.16-7.13(\mathrm{~m}, 2 \mathrm{H}), 4.22(\mathrm{qd}, J=7.1,2.7 \mathrm{~Hz}, 4 \mathrm{H}), 3.45(\mathrm{~d}, J=9.3 \mathrm{~Hz}, 1 \mathrm{H}), 3.25-3.03(\mathrm{~m}, 3 \mathrm{H})$, $2.83-2.69(\mathrm{~m}, 2 \mathrm{H}), 1.28(\mathrm{t}, J=7.1 \mathrm{~Hz}, 6 \mathrm{H}) .{ }^{13} \mathrm{C} \mathrm{NMR}\left(126 \mathrm{MHz}, \mathrm{CDCl}_{3}\right) \delta$ 168.8, 142.1, 126.4, 124.4, 61.4, 56.8, 39.0, 37.3, 14.1; IR (film) 2980, 2840, 1744, 1727, $1476 \mathrm{~cm}^{-1}$; HRMS (ESI ${ }^{+}$ TOF) $\mathrm{m} / \mathrm{z}:\left[\mathrm{M}+\mathrm{H}^{+}\right]^{+}$calcd for $\mathrm{C}_{16} \mathrm{H}_{20} \mathrm{O}_{4} 277.1362$; found 277.1434 .

Diethyl 2-(2,3-dihydro-1H-inden-2-yl)malonate (5a). The title compound was prepared from 2allylphenyl triflate $(267 \mathrm{mg}, 1.0 \mathrm{mmol})$ and diethyl malonate $(183 \mu \mathrm{L}, 1.2 \mathrm{mmol})$ using General Procedure A. The crude material was purified via column chromatography on silica gel using 95:5 hexanes:ethyl acetate as the eluent. This procedure afforded $232 \mathrm{mg}(84 \%)$ of the title compound as a white solid $\left(\mathrm{mp} 33-35^{\circ} \mathrm{C}\right)$. Characterization data were identical to those listed above. 


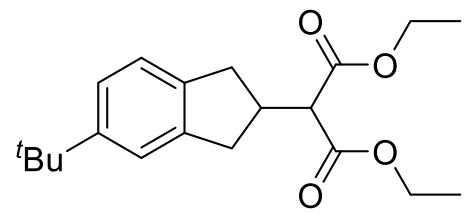

(士)-Diethyl 2-[5-(tert-butyl)-2,3-dihydro-1H-inden-2-yl]malonate (5b). The title compound was prepared from 2-allyl-4-tert-butylphenyl triflate $(64.5 \mathrm{mg}, 0.2 \mathrm{mmol})$ and diethyl malonate (37 $\mu \mathrm{L}, 0.24 \mathrm{mmol})$ using General Procedure A. The crude material was purified via column chromatography on silica gel using 95:5 hexanes:ethyl acetate as the eluent. This procedure afforded $48.3 \mathrm{mg}(77 \%)$ of the title compound as a colorless oil. ${ }^{1} \mathrm{H}$ NMR $\left(400 \mathrm{MHz}, \mathrm{CDCl}_{3}\right) \delta$ $7.24(\mathrm{~s}, 1 \mathrm{H}), 7.20(\mathrm{dd}, J=7.9,1.9 \mathrm{~Hz}, 1 \mathrm{H}), 7.13(\mathrm{~d}, J=7.9 \mathrm{~Hz}, 1 \mathrm{H}), 4.28-4.16(\mathrm{~m}, 4 \mathrm{H}), 3.46$ $(\mathrm{d}, J=8.9 \mathrm{~Hz}, 1 \mathrm{H}), 3.23-3.08(\mathrm{~m}, 3 \mathrm{H}), 2.86-2.66(\mathrm{~m}, 2 \mathrm{H}), 1.32(\mathrm{~s}, 9 \mathrm{H}), 1.31-1.27(\mathrm{~m}, 6 \mathrm{H})$. ${ }^{13} \mathrm{C}$ NMR $\left(126 \mathrm{MHz}, \mathrm{CDCl}_{3}\right) \delta 168.9,149.7,142.1,139.3,124.0,123.7,121.4,61.4,57.1,39.4$, 37.6, 37.0, 34.7, 31.68, 14.3; IR (film) 1748, 1729, $1467 \mathrm{~cm}^{-1}$; HRMS (ESI TOF) m/z: [M + $\left.\mathrm{H}^{+}\right]^{+}$ calcd for $\mathrm{C}_{20} \mathrm{H}_{28} \mathrm{O}_{4} 333.199$; found 333.206.

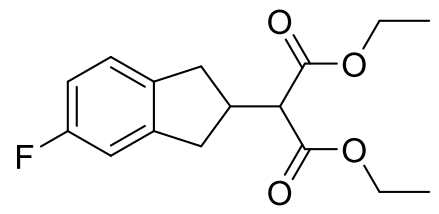

(士)-Diethyl 2-(5-fluoro-2,3-dihydro-1H-inden-2-yl)malonate (5c). The title compound was prepared from 2-allyl-4-fluorophenyl triflate $(56.8 \mathrm{mg}, 0.2 \mathrm{mmol})$ and diethyl malonate $(37 \mu \mathrm{L}$, $0.24 \mathrm{mmol}$ ) using General Procedure A. The crude material was purified via column chromatography on silica gel using 95:5 hexanes:ethyl acetate as the eluent. This procedure afforded $46.5 \mathrm{mg}(79 \%)$ of the title compound as a colorless oil. ${ }^{1} \mathrm{H}$ NMR $\left(500 \mathrm{MHz}, \mathrm{CDCl}_{3}\right) \delta$ 7.09 (dd, $J=8.3,5.2 \mathrm{~Hz}, 1 \mathrm{H}), 6.89-6.83(\mathrm{~m}, 1 \mathrm{H}), 6.82(\mathrm{td}, J=8.9,2.4 \mathrm{~Hz}, 1 \mathrm{H}), 4.25-4.16(\mathrm{~m}$, 4H), $3.44(\mathrm{~d}, J=9.1 \mathrm{~Hz}, 1 \mathrm{H}), 3.24-3.04(\mathrm{~m}, 3 \mathrm{H}), 2.86-2.65(\mathrm{~m}, 2 \mathrm{H}), 1.27(\mathrm{t}, J=7.2 \mathrm{~Hz}, 6 \mathrm{H})$. ${ }^{13} \mathrm{C}$ NMR $\left(126 \mathrm{MHz}, \mathrm{CDCl}_{3}\right) \delta 168.8,163.2,161.2,144.2(\mathrm{~d}, J=8.2 \mathrm{~Hz}), 137.4(\mathrm{~d}, J=2.5 \mathrm{~Hz})$, 
$125.1(\mathrm{~d}, J=8.8 \mathrm{~Hz}), 113.2(\mathrm{~d}, J=22.6 \mathrm{~Hz}), 111.4(\mathrm{~d}, J=21.9 \mathrm{~Hz}), 61.6,56.8,39.7,37.5,37.5$, 36.6, 14.2; IR (film) 2982, 1745, 1727, 1614, 1599, $1483 \mathrm{~cm}^{-1}$; HRMS (ESI+ TOF) m/z: $\left[M+\mathrm{H}^{+}\right]^{+}$ calcd for $\mathrm{C}_{16} \mathrm{H}_{19} \mathrm{FO}_{4} 295.1267$; found 295.134.

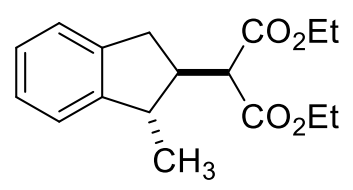

$( \pm)-\left(1 S^{*}, 2 R^{*}\right)$-Diethyl $\quad 2-(1-$ methyl-2,3-dihydro-1H-inden-2-yl)malonate $\quad(5 \mathrm{~d}) . \quad$ The title compound was prepared from 2-(but-3-en-2-yl)phenyl triflate $(56.0 \mathrm{mg}, 0.2 \mathrm{mmol})$, and diethyl malonate $(37 \mu \mathrm{L}, 0.24 \mathrm{mmol})$ using General Procedure A, except with (Brettphos)Pd(allyl)(Cl) (8.6 mg, 0.04 equiv) in place of $\mathrm{Pd}(\mathrm{OAc})_{2}$. The crude material was purified via column chromatography on silica gel using 95:5 hexanes:ethyl acetate as the eluent. This procedure afforded $43.1 \mathrm{mg}(75 \%)$ of the title compound as a colorless oil. The compound was obtained as a $>20: 1$ mixture of diastereomers as judged by ${ }^{1} \mathrm{H}$ NMR analysis. Data are for the major isomer. ${ }^{1} \mathrm{H}$ NMR $\left(401 \mathrm{MHz}, \mathrm{CDCl}_{3}\right) \delta 7.23-7.10(\mathrm{~m}, 4 \mathrm{H}), 4.24-4.15(\mathrm{~m}, 4 \mathrm{H}) 3.51(\mathrm{~d}, J=8.2 \mathrm{~Hz}, 1 \mathrm{H})$, $3.24(\mathrm{dd}, J=16.1,8.1 \mathrm{~Hz}, 1 \mathrm{H}), 3.08(\mathrm{p}, J=6.8 \mathrm{~Hz}, 1 \mathrm{H}), 2.81(\mathrm{dd}, J=16.1,7.3 \mathrm{~Hz}, 1 \mathrm{H}), 2.69(\mathrm{p}$, $J=8.0 \mathrm{~Hz}, 1 \mathrm{H}), 1.32-1.22(\mathrm{~m}, 9 \mathrm{H}) ;{ }^{13} \mathrm{C} \operatorname{NMR}\left(176 \mathrm{MHz}, \mathrm{CDCl}_{3}\right) \delta 169.1,168.8,147.0,141.4$, $126.7,126.66,124.5,123.6,61.51,61.45,55.6,46.9,43.4,35.8,19.8,14.3,14.2$; IR (film) 1741, 1728, $1464 \mathrm{~cm}^{-1}$; HRMS (ESI ${ }^{+}$TOF) m/z: $\left[\mathrm{M}+\mathrm{H}^{+}\right]^{+}$calcd for $\mathrm{C}_{17} \mathrm{H}_{22} \mathrm{O}_{4}$ 291.1518; found 291.1591.

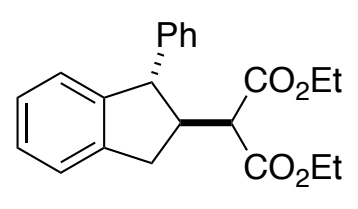

(1S,2S)-Diethyl 2-(1-phenyl-2,3-dihydro-1H-inden-2-yl)malonate (5e). The title compound was prepared from 2-cinnamylphenyl trifluoromethanesulfonate $(75.2 \mathrm{mg}, 0.22 \mathrm{mmol})$ and diethyl malonate $(0.1 \mathrm{~mL}, 0.6 \mathrm{mmol}), \mathrm{Pd}(\mathrm{OAc})_{2}(1.8 \mathrm{mg}, 0.008 \mathrm{mmol}, 0.04$ equiv), Brettphos (6.4 
$\mathrm{mg}, 0.012 \mathrm{mmol}, 0.06$ equiv), with a reaction temperature of $65^{\circ} \mathrm{C}$ for five hours using General Procedure B. The crude material was purified via column chromatography on silica gel using 98:2 -> 95:5 hexanes:ethyl acetate as the eluent. This procedure afforded $39.8 \mathrm{mg}(51 \%)$ of the title compound as a colorless oil. The compound was obtained as a $>20: 1$ mixture of diastereomers as judged by ${ }^{1} \mathrm{H}$ NMR analysis. Data are for the major isomer. ${ }^{1} \mathrm{H}$ NMR $(500$ MHz, Benzene- $\left.d_{6}\right) \delta 7.22-6.97(\mathrm{~m}, 7 \mathrm{H}), 6.93(\mathrm{t}, J=7.4 \mathrm{~Hz}, 1 \mathrm{H}), 6.75(\mathrm{~d}, J=7.5 \mathrm{~Hz}, 1 \mathrm{H}), 4.27$ $(\mathrm{d}, J=9.0 \mathrm{~Hz}, 1 \mathrm{H}), 3.92-3.79(\mathrm{~m}, 2 \mathrm{H}), 3.73-3.52(\mathrm{~m}, 3 \mathrm{H}), 3.45(\mathrm{dd}, J=15.7,7.9 \mathrm{~Hz}, 1 \mathrm{H})$, $3.32-3.22(\mathrm{~m}, 1 \mathrm{H}), 2.96(\mathrm{dd}, J=15.8,8.9 \mathrm{~Hz}, 1 \mathrm{H}), 0.83(\mathrm{t}, J=7.1 \mathrm{~Hz}, 3 \mathrm{H}), 0.73(\mathrm{t}, J=7.1 \mathrm{~Hz}$, $3 \mathrm{H}) .{ }^{13} \mathrm{C}$ NMR $\left(126 \mathrm{MHz}\right.$, Benzene- $\left.d_{6}\right) \delta 168.1,167.9,145.9,143.4,142.0,127.8,127.7,127.6$, 127.5, 126.6, 124.9, 124.2, 60.7, 60.6, 55.0 (2 peaks), 48.9, 36.0, 13.7, 13.4. IR (film) 1730.5, $1601.2 \mathrm{~cm}^{-1}$. HRMS (ESI+ TOF) m/z: $\left[\mathrm{M}+\mathrm{H}^{+}\right]^{+}$calcd for $\mathrm{C}_{22} \mathrm{H}_{24} \mathrm{O}_{4}$ 353.1753; found 353.1747.

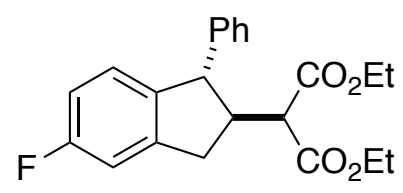

$( \pm)-\left(1 S^{*}, 2 S^{*}\right)$-Diethyl 2-(5-fluoro-1-phenyl-2,3-dihydro-1H-inden-2-yl)malonate (5f). The title compound was prepared from 2-cinnamyl 4-fluorophenyl trifluoromethanesulfonate (64.7 mg, $0.18 \mathrm{mmol})$ and diethyl malonate $(0.1 \mathrm{~mL}, 0.6 \mathrm{mmol})$, palladium acetate $(1.8 \mathrm{mg}, 0.008 \mathrm{mmol}$, 0.04 equiv), Brettphos ( $6.4 \mathrm{mg}, 0.012 \mathrm{mmol}, 0.06$ equiv), with a reaction temperature of $65{ }^{\circ} \mathrm{C}$ for five hours using General Procedure B. The crude material was purified via column chromatography on silica gel using 98:2 -> 95:5 hexanes:ethyl acetate as the eluent. This procedure afforded $31.0 \mathrm{mg}(47 \%)$ of the title compound as a colorless oil. The compound was obtained as a >20:1 mixture of diastereomers as judged by ${ }^{1} \mathrm{H}$ NMR analysis. Data are for the major isomer. ${ }^{1} \mathrm{H}$ NMR $\left(500 \mathrm{MHz}, \mathrm{C}_{6} \mathrm{D}_{6}\right) \delta 7.16-6.94(\mathrm{~m}, 5 \mathrm{H}), 6.69(\mathrm{dd}, J=9.0,2.4 \mathrm{~Hz}, 1 \mathrm{H})$, $6.60(\mathrm{t}, J=8.7 \mathrm{~Hz}, 1 \mathrm{H}), 6.45(\mathrm{~d}, J=8.2 \mathrm{~Hz}, 1 \mathrm{H}), 4.13(\mathrm{~d}, J=8.4 \mathrm{~Hz}, 1 \mathrm{H}), 3.95-3.76(\mathrm{~m}, 2 \mathrm{H})$, $3.65(\mathrm{dd}, J=10.8,7.1 \mathrm{~Hz}, 1 \mathrm{H}), 3.61-3.46(\mathrm{~m}, 2 \mathrm{H}), 3.29-3.17(\mathrm{~m}, 2 \mathrm{H}), 2.80(\mathrm{dd}, J=15.1,8.0$ 
$\mathrm{Hz}, 1 \mathrm{H}), 0.84(\mathrm{t}, J=7.1 \mathrm{~Hz}, 3 \mathrm{H}), 0.73(\mathrm{t}, J=7.1 \mathrm{~Hz}, 3 \mathrm{H}) .{ }^{13} \mathrm{C} N M R\left(126 \mathrm{MHz}, \mathrm{C}_{6} \mathrm{D}_{6}\right) \delta 168.0(\mathrm{~d}$, $J=34 \mathrm{~Hz}), 163.5161 .5,144.1(\mathrm{~d}, J=9 \mathrm{~Hz}), 143.1,141.3(\mathrm{~d}, J=1 \mathrm{~Hz}), 128.8,127.9,126.7$, 126.0, 113.5 (d, $J=25 \mathrm{~Hz}), 111.1(\mathrm{~d}, J=23 \mathrm{~Hz}), 60.8(\mathrm{~d}, J=14 \mathrm{~Hz}), 54.5,54.1,49.2,35.8(2$ peaks), 13.7, 13.4. IR (film) 1728.0, 1600.2, $1483.7 \mathrm{~cm}^{-1}$. HRMS (ESI+ TOF) $\mathrm{m} / \mathrm{z}:\left[\mathrm{M}+\mathrm{H}^{+}\right]^{+}$ calcd for $\mathrm{C}_{22} \mathrm{H}_{23} \mathrm{FO}_{4} 371.1659$; found 371.1653.

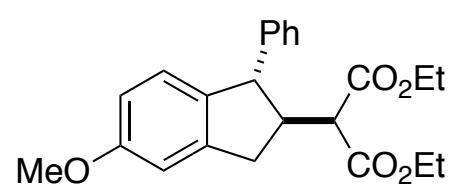

( \pm )-(1S,2S)-Diethyl 2-(5-methoxy-1-phenyl-2,3-dihydro-1H-inden-2-yl)malonate (5g). The title compound was prepared from 2-cinnamyl 4-methoxyphenyl trifluoromethanesulfonate (81.6 $\mathrm{mg}, 0.23 \mathrm{mmol})$, diethyl malonate $(0.1 \mathrm{~mL}, 0.6 \mathrm{mmol})$, palladium acetate $(1.8 \mathrm{mg}, 0.008 \mathrm{mmol}$, 0.04 equiv), Brettphos (6.4 mg, $0.012 \mathrm{mmol}, 0.06$ equiv), with a reaction temperature of $65{ }^{\circ} \mathrm{C}$ for five hours using General Procedure B. The crude material was purified via column chromatography on silica gel using 98:2 -> 95:5 hexanes:ethyl acetate as the eluent. This procedure afforded $29.2 \mathrm{mg} \mathrm{(35 \% )}$ of the title compound as a colorless oil. The compound was obtained as a $>20: 1$ mixture of diastereomers as judged by ${ }^{1} \mathrm{H}$ NMR analysis. Data are for the major isomer. ${ }^{1} \mathrm{H}$ NMR $\left(500 \mathrm{MHz}, \mathrm{C}_{6} \mathrm{D}_{6}\right) \delta 7.19(\mathrm{~d}, J=7.2 \mathrm{~Hz}, 2 \mathrm{H}), 7.10(\mathrm{~s}, 3 \mathrm{H}), 7.01(\mathrm{~s}, 1 \mathrm{H})$, $6.69(\mathrm{~d}, J=19.9 \mathrm{~Hz}, 2 \mathrm{H}), 6.61(\mathrm{~d}, J=10.3 \mathrm{~Hz}, 1 \mathrm{H}), 4.26(\mathrm{~d}, J=8.5 \mathrm{~Hz}, 1 \mathrm{H}), 3.97-3.78(\mathrm{~m}$, 2H), $3.76-3.53(\mathrm{~m}, 3 \mathrm{H}), 3.49-3.36(\mathrm{~m}, 1 \mathrm{H}), 3.30(\mathrm{~s}, 3 \mathrm{H}), 3.04-2.87(\mathrm{~m}, 1 \mathrm{H}), 0.84(\mathrm{t}, J=7.1$ $\mathrm{Hz}, 3 \mathrm{H}), 0.74(\mathrm{t}, J=7.1 \mathrm{~Hz}, 3 \mathrm{H}) .{ }^{13} \mathrm{C}$ NMR $\left(126 \mathrm{MHz}, \mathrm{C}_{6} \mathrm{D}_{6}\right) \delta 168.2,167.2,159.5,143.9,143.4$, 137.8, 128.8, 128.3, 127.9, 127.8, 126.5, 125.6, 113.0, 109.5, 60.7, 54.8, 54.6, 54.3, 49.4, 36.1, 13.7. IR (film) 1727.7, $1608.5,1588.8, \mathrm{~cm}^{-1}$. HRMS (ESI+ TOF) m/z: $\left[\mathrm{M}+\mathrm{H}^{+}\right]^{+}$calcd for $\mathrm{C}_{23} \mathrm{H}_{26} \mathrm{O}_{5}$ 383.1859; found 383.1853. 


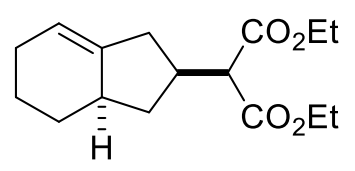

$( \pm)-\left(2 S^{*}, 3 a R^{*}\right)$-Diethyl $\quad 2-(2,3,3 a, 4,5,6$-hexahydro-1H-inden-2-yl)malonate $(6 a)$. The title compound was prepared from 6-allylcyclohex-1-en-1-yl triflate $(54.1 \mathrm{mg}, 0.2 \mathrm{mmol})$ and diethyl malonate $(37 \mu \mathrm{L}, 0.24 \mathrm{mmol})$ using General Procedure A. The crude material was purified via column chromatography on silica gel using 98:2 hexanes:ethyl acetate as the eluent. This procedure afforded $45.4 \mathrm{mg}(84 \%)$ of the title compound as a colorless oil. The compound was obtained as a $>20: 1$ mixture of diastereomers as judged by ${ }^{1} \mathrm{H}$ NMR analysis. Data are for the major isomer. ${ }^{1} \mathrm{H}$ NMR $\left(500 \mathrm{MHz}, \mathrm{CDCl}_{3}\right) \delta 5.37(\mathrm{~s}, 1 \mathrm{H}), 4.19(\mathrm{qd}, \mathrm{J}=7.1,3.1 \mathrm{~Hz}, 4 \mathrm{H}), 3.17(\mathrm{~d}$, $J=9.9 \mathrm{~Hz}, 1 \mathrm{H}), 2.70-2.52(\mathrm{~m}, 2 \mathrm{H}), 2.22(\mathrm{br} \mathrm{s}, 1 \mathrm{H}), 2.11-2.01(\mathrm{~m}, 1 \mathrm{H}), 2.03-1.91(\mathrm{~m}, 4 \mathrm{H})$, 1.77 (ddd, $J=12.3,6.0,3.2 \mathrm{~Hz}, 1 \mathrm{H}), 1.43(\mathrm{~m}, 1 \mathrm{H}), 1.26(\mathrm{td}, J=7.1,1.9 \mathrm{~Hz}, 6 \mathrm{H}), 0.99(\mathrm{~m}, 1 \mathrm{H})$, $0.88(\mathrm{q}, J=11.5 \mathrm{~Hz}, 1 \mathrm{H}) ;{ }^{13} \mathrm{C}$ NMR $\left(125 \mathrm{MHz}, \mathrm{CDCl}_{3}\right) \delta 169.1,169.0,142.8,118.1,61.4,57.8$ 41.0, 38.6, 37.1, 35.3, 28.9, 25.3, 22.5, 14.3; IR (film) 1730, $1029 \mathrm{~cm}^{-1}$; HRMS (ESI+ TOF) m/z: $\left[\mathrm{M}+\mathrm{H}^{+}\right]^{+}$calcd for $\mathrm{C}_{16} \mathrm{H}_{24} \mathrm{O}_{4} 281.1675$; found 281.1747 .

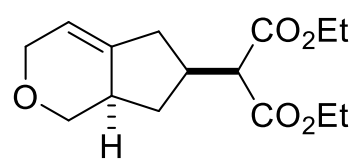

$( \pm)-\left(6 S^{*}, 7 a R^{*}\right)-$ Diethyl 2-(1,3,5,6,7,7a-hexahydrocyclopenta[c]pyran-6-yl)malonate (6b). The title compound was prepared from 3-allyl-3,6-dihydro-2H-pyran-4-yl triflate $(54.4 \mathrm{mg}, 0.2 \mathrm{mmol})$ and diethyl malonate (37 $\mu \mathrm{L}, 0.24 \mathrm{mmol})$ using General Procedure A. The crude material was purified via column chromatography on silica gel using 95:5 -> 90:10 hexanes:ethyl acetate as the eluent. This procedure afforded $53.6 \mathrm{mg} \mathrm{(95 \% )}$ of the title compound as a colorless oil. The compound was obtained as a $>20: 1$ mixture of diastereomers as judged by ${ }^{1} \mathrm{H}$ NMR analysis. Data are for the major isomer. ${ }^{1} \mathrm{H}$ NMR $\left(500 \mathrm{MHz} \mathrm{CDCl}_{3}\right) \delta 5.40(\mathrm{~s}, 1 \mathrm{H}), 4.25-4.09(\mathrm{~m}, 6 \mathrm{H})$, $4.09-3.99(\mathrm{~m}, 1 \mathrm{H}), 3.19(\mathrm{~d}, J=9.2 \mathrm{~Hz}, 1 \mathrm{H}), 3.03(\mathrm{t}, J=10.2 \mathrm{~Hz}, 1 \mathrm{H}), 2.75-2.63(\mathrm{~m}, 2 \mathrm{H}), 2.56$ 
(br s, 1H), $2.11-1.96(\mathrm{~m}, 2 \mathrm{H}), 1.26(\mathrm{t}, J=7.1 \mathrm{~Hz}, 6 \mathrm{H}), 0.87(\mathrm{q}, J=11.5 \mathrm{~Hz}, 1 \mathrm{H}) ;{ }^{13} \mathrm{C}$ NMR $(101$ $\left.\mathrm{MHz}_{\mathrm{CDCl}}\right) \delta 168.8,140.8,116.8,69.3,65.1,61.5,57.4,39.8,37.1,34.7,34.0,14.3 ; \mathrm{IR}$ (film) 1750, 1727, $1461 \mathrm{~cm}^{-1}$; HRMS (ESI TOF) m/z: $\left[\mathrm{M}+\mathrm{H}^{+}\right]^{+}$calcd for $\mathrm{C}_{15} \mathrm{H}_{22} \mathrm{O}_{5}$ 305.1359; found 305.1362.

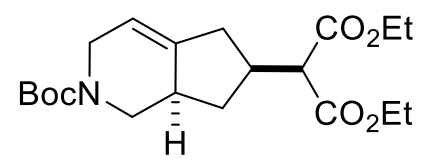

$( \pm)-\left(6 S^{*}, 7 \mathrm{a} R^{*}\right)-$ Diethyl

2-[2-(tert-butoxycarbonyl)-2,3,5,6,7,7a-hexahydro-1Hcyclopenta[c]pyridin-6-yl]malonate (6c). The title compound was prepared from tert-butyl 3allyl-4-\{[(trifluoromethyl)sulfonyl]oxy\}-3,6-dihydropyridine-1(2H)-carboxylate $(67.1 \mathrm{mg}, 0.2 \mathrm{mmol})$ and diethyl malonate (37 $\mu \mathrm{L}, 0.24 \mathrm{mmol})$ using General Procedure A. The crude material was purified via column chromatography on silica gel using 25:75 hexanes:dichloromethane as the eluent. This procedure afforded $52.3 \mathrm{mg}(76 \%)$ of the title compound as a colorless oil. Characterization data are for a mixture of rotamers; broadening is due to slow interconversion on the NMR time scale. ${ }^{1} \mathrm{H}$ NMR $\left(500 \mathrm{MHz}, \mathrm{C}_{6} \mathrm{D}_{6}\right) \delta 5.09(\mathrm{~s}, 1 \mathrm{H}), 4.57-4.15(\mathrm{~m}, 2 \mathrm{H}), 4.11-$ $3.82(\mathrm{br} \mathrm{m}, 4 \mathrm{H}), 3.43-3.37(\mathrm{~m}, 1 \mathrm{H}), 3.18-3.15(\mathrm{~m}, 1 \mathrm{H}), 2.81-2.53(\mathrm{~m}, 2 \mathrm{H}), 2.25(\mathrm{br} \mathrm{s}, 1 \mathrm{H})$, $2.21-2.12(\mathrm{~m}, 1 \mathrm{H}), 2.07-1.95(\mathrm{br} m, 1 \mathrm{H}), 1.94-1.81(\mathrm{~m}, 1 \mathrm{H}), 1.55-1.38(\mathrm{~m}, 9 \mathrm{H}), 1.04-$ $0.90(\mathrm{~m}, 6 \mathrm{H}), 0.86-0.74(\mathrm{~m}, 1 \mathrm{H}) .{ }^{13} \mathrm{C}$ NMR $\left(100 \mathrm{MHz}, \mathrm{CDCl}_{3}\right) \delta$ 168.7, 79.7, 61.5, 57.3, 40.2, 37.5, 35.1, 34.8, 28.6, 28.4, 14.2; IR (film) 1751, 1729, $\left.1693 \mathrm{~cm}^{-1} ; \mathrm{HRMS}^{\left(E S I^{+}\right.} \mathrm{TOF}\right) \mathrm{m} / \mathrm{z}$ : 404.2044 (404.2044 calcd for $\mathrm{C}_{20} \mathrm{H}_{31} \mathrm{NO}_{6}, \mathrm{M}+\mathrm{Na}^{+}$). HRMS (ESI ${ }^{+}$TOF) $\mathrm{m} / \mathrm{z}:\left[\mathrm{M}+\mathrm{Na}^{+}\right]^{+}$calcd for $\mathrm{C}_{20} \mathrm{H}_{31} \mathrm{NO}_{6} 404.2044$; found 404.2044 .<smiles>CCOC(=O)C(C1CC2=CCNCC2C1)C(OCC)OCC</smiles>

$( \pm)-\left(6 S^{*}, 7 a R^{\star}\right)$-diethyl $\quad 2-(2,3,5,6,7,7$ a-hexahydro-1H-cyclopenta[c]pyridin-6-yl)malonate (S9). In order to confirm the connectivity and stereochemistry of $\mathbf{6 c}$, the boc group was cleaved 
by addition of trifluoroacetic acid (3 drops) to a solution of $6 \mathbf{c}$ in dichloromethane; the resulting solution was stirred for 1 hour at rt. The solvent and excess trifluoroacetic acid were removed under reduced pressure and the product was washed with $\mathrm{NaHCO}_{3}$, brine, and dried with $\mathrm{MgSO}_{4}$. This procedure afforded $34.3 \mathrm{mg}(89 \%)$ of the title compound as a colorless oil. This material was judged to be a $>20: 1$ mixture of diastereomers by $1 \mathrm{H}$ NMR analysis. Data are for the major isomer. ${ }^{1} \mathrm{H}$ NMR $\left(400 \mathrm{MHz}, \mathrm{CDCl}_{3}\right) \delta 5.38(\mathrm{~s}, 1 \mathrm{H}), 4.18(\mathrm{q}, \mathrm{J}=7.1 \mathrm{~Hz}, 4 \mathrm{H}), 3.37-3.29$ (m, 2H), $3.18(\mathrm{~d}, J=9.5 \mathrm{~Hz}, 1 \mathrm{H}), 2.73-2.59(\mathrm{~m}, 2 \mathrm{H}), 2.40(\mathrm{br} \mathrm{s}, 1 \mathrm{H}), 2.33-2.23(\mathrm{~m}, 1 \mathrm{H}), 2.11$ - $1.97(\mathrm{~m}, 2 \mathrm{H}), 1.49-1.37(\mathrm{~m}, 1 \mathrm{H}), 1.26(\mathrm{t}, J=7.1 \mathrm{~Hz}, 6 \mathrm{H}), 0.88(\mathrm{q}, J=11.3 \mathrm{~Hz}, 1 \mathrm{H}) ;{ }^{13} \mathrm{C}$ NMR $\left(100 \mathrm{MHz}, \mathrm{CDCl}_{3}\right) \delta 168.9,168.8,141.8,116.8,61.4,57.5,48.3,44.5,40.4,36.9,35.7,35.0$ 14.2; IR (film) 2983, 1782, 1730, $1148 \mathrm{~cm}^{-1}$; HRMS (ESI ${ }^{+}$TOF) m/z: $\left[\mathrm{M}+\mathrm{H}^{+}\right]^{+}$calcd for $\mathrm{C}_{15} \mathrm{H}_{23} \mathrm{NO}_{4} 282.1700$; found 282.1706.

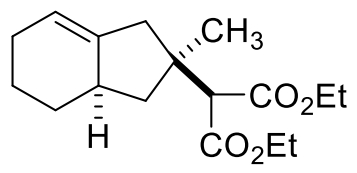

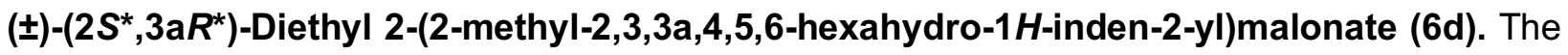
title compound was prepared from 6-(2-methylallyl)cyclohex-1-en-1-yl triflate (54.1 mg, 0.2 $\mathrm{mmol}$ ) and diethyl malonate $(37 \mu \mathrm{L}, 0.24 \mathrm{mmol})$ using General Procedure A. The crude material was purified via column chromatography on silica gel using 95:5 hexanes:ethyl acetate as the eluent. This procedure afforded $55.6 \mathrm{mg}(94 \%)$ of the title compound as a colorless oil. The compound was obtained as a 9:1 mixture of diastereomers as judged by ${ }^{1} \mathrm{H}$ NMR analysis. Data are for the major isomer. ${ }^{1} \mathrm{H}$ NMR $\left(500 \mathrm{MHz} \mathrm{CDCl}_{3}\right) \delta 5.35(\mathrm{~s}, 1 \mathrm{H}), 4.17(\mathrm{q}, \mathrm{J}=7.1 \mathrm{~Hz}, 4 \mathrm{H})$, $3.32(\mathrm{~s}, 1 \mathrm{H}), 2.48(\mathrm{~d}, J=17.0 \mathrm{~Hz}, 1 \mathrm{H}), 2.39(\mathrm{br} \mathrm{s}, 1 \mathrm{H}), 2.28(\mathrm{~d}, J=17.0 \mathrm{~Hz}, 1 \mathrm{H}), 2.02-1.90(\mathrm{~m}$, 3H), $1.87-1.73(\mathrm{~m}, 2 \mathrm{H}), 1.45$ (tddd, $J=13.4,10.3,7.5,2.9 \mathrm{~Hz}, 1 \mathrm{H}), 1.28-1.21(\mathrm{~m}, 10 \mathrm{H}), 1.03$ - $0.92(\mathrm{~m}, 1 \mathrm{H}) ;{ }^{13} \mathrm{C}$ NMR $\left(126 \mathrm{MHz}, \mathrm{CDCl}_{3}\right) \delta 168.8,143.1,118.1,61.7,61.3,61.04,61.03$, 45.9, 44.1, 41.6, 38.7, 38.6, 29.0, 28.9, 25.3, 24.9, 22.6, 22.5, 14.3; IR (film) 1753, $1729 \mathrm{~cm}^{-1}$; HRMS (ESI ${ }^{+}$TOF) m/z: $\left[\mathrm{M}+\mathrm{Na}^{+}\right]^{+}$calcd for $\mathrm{C}_{17} \mathrm{H}_{26} \mathrm{O}_{4} 317.1723$; found 317.1729 . 


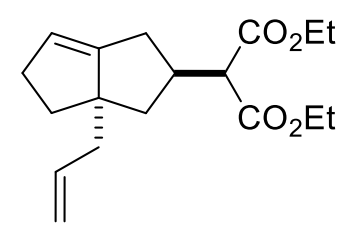

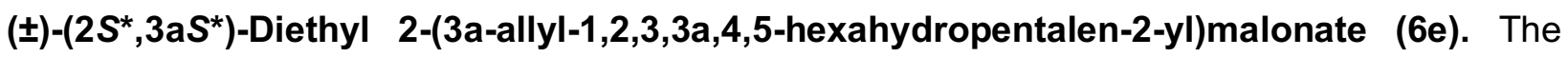
title compound was prepared from 5,5-diallylcyclopent-1-en-1-yl triflate (60 mg, $0.2 \mathrm{mmol})$ and diethyl malonate $(37 \mu \mathrm{L}, 0.24 \mathrm{mmol})$ using General Procedure A. The crude material was purified via column chromatography on silica gel using 95:5 hexanes:ethyl acetate as the eluent. This procedure afforded $56.6 \mathrm{mg}(92 \%)$ of the title compound as a colorless oil. The compound was obtained as a $>20: 1$ mixture of diastereomers as judged by ${ }^{1} \mathrm{H}$ NMR analysis. Data are for the major isomer. ${ }^{1} \mathrm{H}$ NMR $\left(700 \mathrm{MHz}, \mathrm{CDCl}_{3}\right) \delta 5.79$ (dddd, $\left.J=16.9,10.1,7.9,6.6 \mathrm{~Hz}, 1 \mathrm{H}\right), 5.22$ (s, 1H), $5.05(\mathrm{~d}, J=17.0 \mathrm{~Hz}, 1 \mathrm{H}), 5.02(\mathrm{~d}, J=10.1 \mathrm{~Hz}, 1 \mathrm{H}), 4.22-4.12(\mathrm{~m}, 4 \mathrm{H}), 3.25(\mathrm{~d}, J=$ $10.3 \mathrm{~Hz}, 1 \mathrm{H}), 3.09$ (qt, $J=10.3,6.4 \mathrm{~Hz}, 1 \mathrm{H}), 2.63-2.50(\mathrm{~m}, 2 \mathrm{H}), 2.42-2.36(\mathrm{~m}, 2 \mathrm{H}), 2.12$ (dd, $J=13.8,6.6 \mathrm{~Hz}, 1 \mathrm{H}), 2.07(\mathrm{dd}, J=13.8,8.0 \mathrm{~Hz}, 1 \mathrm{H}), 1.98-1.92(\mathrm{~m}, 2 \mathrm{H}), 1.56(\mathrm{dt}, J=12.4,9.5$ $\mathrm{Hz}, 1 \mathrm{H}), 1.25$ (td, $J=7.1,4.4 \mathrm{~Hz}, 6 \mathrm{H}), 1.03(\mathrm{dd}, J=12.0,10.5 \mathrm{~Hz}, 1 \mathrm{H}) ;{ }^{13} \mathrm{C}$ NMR $(176 \mathrm{MHz}$, $\left.\mathrm{CDCl}_{3}\right) \delta 168.9,168.8,153.9,136.1,119.1,116.9,61.41,61.37,59.3,58.2,41.5,40.3,39.9$ 36.9, 36.1, 28.2, 14.3; IR (film) 1732, $1150 \mathrm{~cm}^{-1}$; HRMS (ESI TOF) m/z: $\left[\mathrm{M}+\mathrm{H}^{+}\right]^{+}$calcd for $\mathrm{C}_{18} \mathrm{H}_{26} \mathrm{O}_{4} 307.1904$; found 307.1909.

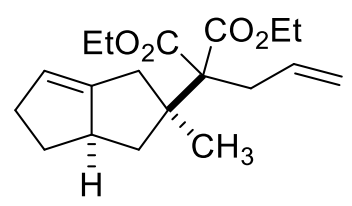

$( \pm)-\left(2 S^{*}, 3 a R^{\star}\right)$-Diethyl 2-allyl-2-(2-methyl-1,2,3,3a,4,5-hexahydropentalen-2-yl)malonate

(6f). The title compound was prepared from (methylallyl)cyclopent-1-en-1-yl triflate (54.0 mg, 0.2 $\mathrm{mmol})$, and diethyl allylmalonate $(48 \mu \mathrm{L}, 0.24 \mathrm{mmol})$ using General Procedure A, except with RuPhos (5.6 mg, $0.012 \mathrm{mmol}$ ) as ligand. The crude material was purified via column chromatography on silica gel using 95:5 hexanes:ethyl acetate as the eluent. This procedure 
afforded $40.8 \mathrm{mg}(63 \%)$ of the title compound as a colorless oil. The compound was obtained as a 19:1 mixture of diastereomers as judged by ${ }^{1} \mathrm{H}$ NMR analysis. NOTE: The alkene was observed to isomerize in $\mathrm{CDCl}_{3}$ to a 4:1 mixture of regioisomers. Data are for the major isomer. ${ }^{1} \mathrm{H}$ NMR $\left(401 \mathrm{MHz}, \mathrm{CDCl}_{3}\right) \delta 5.90(\mathrm{ddt}, J=17.1,10.1,7.1 \mathrm{~Hz}, 1 \mathrm{H}), 5.22(\mathrm{~s}, 1 \mathrm{H}), 5.14-4.92(\mathrm{~m}$, 2H), $4.15(\mathrm{~m}, 4 \mathrm{H}), 3.12-2.95(\mathrm{br} \mathrm{m}, 1 \mathrm{H}), 2.82-2.38(\mathrm{~m}, 5 \mathrm{H}), 2.10(\mathrm{dtd}, J=12.0,6.8,1.2 \mathrm{~Hz}$, 1H), 1.98 (ddd, $J=17.4,4.5,2.3 \mathrm{~Hz}, 1 \mathrm{H}), 1.83-1.60(\mathrm{~m}, 2 \mathrm{H}), 1.50-1.33(\mathrm{~m}, 1 \mathrm{H}), 1.24(\mathrm{q}, J=$ $7.3 \mathrm{~Hz}, 6 \mathrm{H}), 1.15(\mathrm{~s}, 3 \mathrm{H}) ;{ }^{13} \mathrm{C} \mathrm{NMR}\left(126 \mathrm{MHz}, \mathrm{CDCl}_{3}\right) \delta 170.83,170.79,152.5,135.2,118.0$, 117.5, 110.0, 65.5, 60.7, 51.9, 49.4, 43.2, 37.7, 37.3, 37.0, 32.4, 26.5, 14.1; IR (film) 1741, 1723, $1206 \mathrm{~cm}^{-1}$; HRMS (ESI TOF) m/z: [M + Na $]^{+}$calcd for $\mathrm{C}_{19} \mathrm{H}_{28} \mathrm{O}_{4} 343.199$; found 343.188.

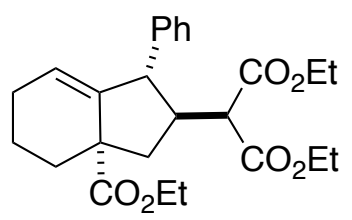

(士)-(1S,2S,3aS)-Diethyl 2-[3a-(ethoxycarbonyl)-1-phenyl-2,3,3a,4,5,6-hexahydro-1H-inden-

2-yl] malonate (6g). The title compound was prepared from ethyl 1-cinnamyl-2$\{[$ (trifluoromethyl)sulfonyl]oxy\}cyclohex-2-ene-1-carboxylate $(83.0 \mathrm{mg}, 0.20 \mathrm{mmol})$ and diethyl malonate $(0.1 \mathrm{~mL}, 0.6 \mathrm{mmol}), \mathrm{Pd}(\mathrm{acac})_{2}(2.4 \mathrm{mg}, 0.008 \mathrm{mmol}, 0.04$ equiv), Sphos (4.9 mg, $0.012 \mathrm{mmol}, 0.06$ equiv), at $95{ }^{\circ} \mathrm{C}$ for fourteen hours using General Procedure B. The crude material was purified via column chromatography on silica gel using 95:5 -> 90:10 hexanes:ethyl acetate as the eluent. This procedure afforded $62.5 \mathrm{mg}(73 \%)$ of the title compound as a colorless oil. The compound was obtained as a $>20: 1$ mixture of diastereomers as judged by ${ }^{1} \mathrm{H}$ NMR analysis. Data are for the major isomer. ${ }^{1} \mathrm{H}$ NMR $\left(500 \mathrm{MHz}, \mathrm{C}_{6} \mathrm{D}_{6}\right) \delta 7.64$ (d, $J=7.6 \mathrm{~Hz}, 2 \mathrm{H}), 7.21-7.09(\mathrm{~m}, 2 \mathrm{H}), 7.02(\mathrm{t}, J=7.3 \mathrm{~Hz}, 1 \mathrm{H}), 5.33(\mathrm{q}, J=3.2 \mathrm{~Hz}, 1 \mathrm{H}), 4.06-$ $3.78(\mathrm{~m}, 4 \mathrm{H}), 3.59(\mathrm{dq}, J=10.5,7.0 \mathrm{~Hz}, 1 \mathrm{H}), 3.49(\mathrm{~d}, J=6.9 \mathrm{~Hz}, 1 \mathrm{H}), 3.44(\mathrm{dq}, J=10.8,7.2$ $\mathrm{Hz}, 1 \mathrm{H}), 3.14-2.95(\mathrm{~m}, 2 \mathrm{H}), 2.37(\mathrm{dt}, J=12.6,3.5 \mathrm{~Hz}, 1 \mathrm{H}), 1.94-1.82(\mathrm{~m}, 1 \mathrm{H}), 1.73(\mathrm{dtd}, J=$ 18.3, 7.4, 3.8 Hz, 1H), $1.61-1.38(\mathrm{~m}, 3 \mathrm{H}), 1.37-1.22(\mathrm{~m}, 1 \mathrm{H}), 1.12(\mathrm{td}, J=13.1,3.8 \mathrm{~Hz}, 1 \mathrm{H})$, 
$1.01-0.91(\mathrm{~m}, 3 \mathrm{H}), 0.84(\mathrm{dt}, J=17.9,7.1 \mathrm{~Hz}, 3 \mathrm{H}), 0.70(\mathrm{t}, J=7.1 \mathrm{~Hz}, 3 \mathrm{H}) .{ }^{13} \mathrm{C}$ NMR $(126 \mathrm{MHz}$, $\left.\mathrm{C}_{6} \mathrm{D}_{6}\right) \delta 175.1,167.9,167.6,144.5,143.3,129.4,129.3,127.5,126.3,123.8,60.8,60.6,54.2$, 53.5, 53.4, 44.6, 41.9, 33.0, 24.5, 19.2, 13.9, 13.7, 13.3. IR (film) 1724.1, 1605.6, $1446.6 \mathrm{~cm}^{-1}$. HRMS (ESI ${ }^{+}$TOF) $\mathrm{m} / \mathrm{z}:\left[\mathrm{M}+\mathrm{H}^{+}\right]^{+}$calcd for $\mathrm{C}_{25} \mathrm{H}_{32} \mathrm{O}_{6} 429.2277$; found 429.2272 .

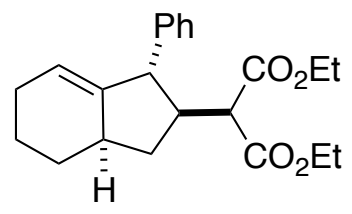

( \pm )-(1R,2S,3aR)-Diethyl 2-(1-phenyl-2,3,3a,4,5,6-hexahydro-1H-inden-2-yl)malonate (6h).

The title compound was prepared from 6-cinnamylcyclohex-1-en-1-yl trifluoromethanesulfonate (69.0 mg, $0.20 \mathrm{mmol})$ and diethyl malonate $(0.1 \mathrm{~mL}, 0.6 \mathrm{mmol}), \mathrm{Pd}(\mathrm{acac})_{2}(2.4 \mathrm{mg}, 0.008 \mathrm{mmol}$, 0.04 equiv), Sphos ( $4.9 \mathrm{mg}, 0.012 \mathrm{mmol}, 0.06$ equiv), at $95^{\circ} \mathrm{C}$ for fourteen hours using General Procedure B. The crude material was purified via column chromatography on silica gel using 98:2 hexanes:ethyl acetate as the eluent. This procedure afforded $54.1 \mathrm{mg}(76 \%)$ of the title compound as a colorless oil. The compound was obtained as a $>20: 1$ mixture of diastereomers as judged by ${ }^{1} \mathrm{H}$ NMR analysis. Data are for the major isomer. ${ }^{1} \mathrm{H}$ NMR $\left(500 \mathrm{MHz}, \mathrm{CDCl}_{3}\right) \delta 7.32$ $-7.21(\mathrm{~m}, 2 \mathrm{H}), 7.21-7.11(\mathrm{~m}, 3 \mathrm{H}), 5.23(\mathrm{p}, J=2.9 \mathrm{~Hz}, 1 \mathrm{H}), 4.15(\mathrm{p}, J=7.0 \mathrm{~Hz}, 2 \mathrm{H}), 3.82(\mathrm{dq}$, $J=10.7,7.1 \mathrm{~Hz}, 1 \mathrm{H}), 3.63(\mathrm{dq}, J=10.8,7.2 \mathrm{~Hz}, 1 \mathrm{H}), 3.40(\mathrm{dt}, J=9.6,2.6 \mathrm{~Hz}, 1 \mathrm{H}), 3.34(\mathrm{~d}, J=$ $8.1 \mathrm{~Hz}, 1 \mathrm{H}), 2.78-2.66(\mathrm{~m}, 1 \mathrm{H}), 2.58-2.45(\mathrm{~m}, 1 \mathrm{H}), 2.31(\mathrm{dt}, J=12.0,6.1 \mathrm{~Hz}, 1 \mathrm{H}), 2.09-$ $1.88(\mathrm{~m}, 3 \mathrm{H}), 1.79(\mathrm{dt}, J=12.7,3.9 \mathrm{~Hz}, 1 \mathrm{H}), 1.51-1.38(\mathrm{~m}, 1 \mathrm{H}), 1.24(\mathrm{t}, J=7.1 \mathrm{~Hz}, 3 \mathrm{H}), 1.16-$ 0.95 (m, 5H). ${ }^{13} \mathrm{C}$ NMR $\left(126 \mathrm{MHz}, \mathrm{CDCl}_{3}\right) \delta 168.6,168.3,147.6,145.2,128.2,128.1,126.1$, 120.3, 61.2, 61.0, 55.5, 53.1, 46.4, 41.3, 37.5, 28.8, 25.1, 22.2, 14.1, 13.7. IR (film) 1730.9, $1601.5 \mathrm{~cm}^{-1}$. HRMS (ESI+ TOF) m/z: $\left[\mathrm{M}+\mathrm{H}^{+}\right]^{+}$calcd for $\mathrm{C}_{22} \mathrm{H}_{28} \mathrm{O}_{4} 357.2066$; found 357.2060 .

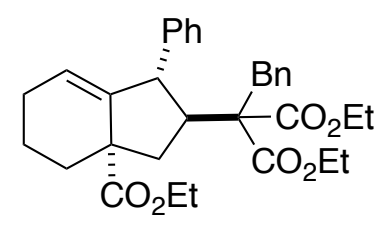




\section{(士)-(1S,2S,3aS)-Diethyl 2-benzyl-2-[3a-(ethoxycarbonyl-1-phenyl-2,3,3a,4,5,6-hexahydro-}

1H-inden-2-yl]malonate (6i). The title compound was prepared from ethyl 1-cinnamyl-2$\{[($ trifluoromethyl)sulfonyl]oxy\}cyclohex-2-ene-1-carboxylate $(85.4 \mathrm{mg}, 0.20 \mathrm{mmol})$ and benzyl diethyl malonate $(0.1 \mathrm{~mL}, 0.43 \mathrm{mmol}), \mathrm{Pd}(\mathrm{acac})_{2}(2.4 \mathrm{mg}, 0.008 \mathrm{mmol}, 0.04$ equiv), Sphos (4.9 mg, 0.012 mmol, 0.06 equiv), for 14 hours using General Procedure B, except with xylenes as solvent at $110{ }^{\circ} \mathrm{C}$. The crude material was purified via column chromatography on silica gel using 98:2 -> 95:5 hexanes:ethyl acetate as the eluent. This procedure afforded $53.3 \mathrm{mg}(51 \%)$ of the title compound as a colorless oil. The compound was obtained as a $>20: 1$ mixture of diastereomers as judged by ${ }^{1} \mathrm{H}$ NMR analysis. Data are for the major isomer. ${ }^{1} \mathrm{H}$ NMR $(500$ $\left.\mathrm{MHz}, \mathrm{CDCl}_{3}\right) \delta 7.38-7.21(\mathrm{~m}, 4 \mathrm{H}), 7.16-7.08(\mathrm{~m}, 3 \mathrm{H}), 7.04(\mathrm{dd}, J=7.1,2.4 \mathrm{~Hz}, 2 \mathrm{H}), 5.43(\mathrm{~d}$, $J=2.5 \mathrm{~Hz}, 1 \mathrm{H}), 4.15(\mathrm{dd}, J=29.8,7.1 \mathrm{~Hz}, 2 \mathrm{H}), 4.03-3.84(\mathrm{~m}, 2 \mathrm{H}), 3.70(\mathrm{~s}, 2 \mathrm{H}), 3.10(\mathrm{~d}, J=$ $13.9 \mathrm{~Hz}, 1 \mathrm{H}), 3.01(\mathrm{~d}, J=14.1 \mathrm{~Hz}, 1 \mathrm{H})$, ), $2.83(\mathrm{dd}, J=12.6,6.2 \mathrm{~Hz}, 1 \mathrm{H}), 2.27(\mathrm{dt}, J=12.7,3.5$ $\mathrm{Hz}, 1 \mathrm{H}), 2.18-1.90(\mathrm{~m}, 2 \mathrm{H}), 1.73-1.46(\mathrm{~m}, 2 \mathrm{H}), 1.25(\mathrm{dt}, J=14.2,8.3 \mathrm{~Hz}, 5 \mathrm{H}), 1.11(\mathrm{t}, J=7.1$ $\mathrm{Hz}, 3 \mathrm{H}), 0.97(\mathrm{t}, J=7.2 \mathrm{~Hz}, 3 \mathrm{H}), 0.88(\mathrm{t}, J=6.7 \mathrm{~Hz}, 3 \mathrm{H}) .{ }^{13} \mathrm{C} \mathrm{NMR}\left(126 \mathrm{MHz}, \mathrm{CDCl}_{3}\right) \delta 175.9$, $170.0,169.9,144.9,144.5,137.0,130.2,128.6,128.0,127.8,126.6,123.4,62.2,60.9,60.8$ 52.4, 51.8, 48.2, 40.9, 40.4, 33.6, 31.6, 24.5, 22.6, 18.9, 14.1, 13.9, 13.7. IR (film) 1720.8, 1601.5, $1495.7 \mathrm{~cm}^{-1}$. HRMS (ESI ${ }^{+}$TOF) $\mathrm{m} / \mathrm{z}$ : $\left[\mathrm{M}+\mathrm{H}^{+}\right]^{+}$calcd for $\mathrm{C}_{32} \mathrm{H}_{38} \mathrm{O}_{6} 519.2747$; found 519.2741

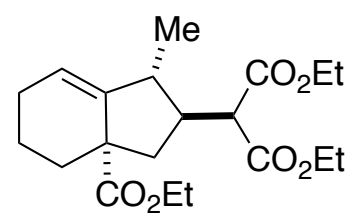

(士)-(1R,2S,3aS)-Diethyl 2-[3a-(ethoxycarbonyl)-1-methyl-2,3,3a,4,5,6-hexahydro-1H-inden2-yl]malonate (6j). The title compound was prepared from ethyl (E)-1-(but-2-en-1-yl)-2$\{[$ (trifluoromethyl)sulfonyl]oxy\}cyclohex-2-ene-1-carboxylate $(73.5 \mathrm{mg}, 0.21 \mathrm{mmol}$ ) and diethyl malonate $(0.1 \mathrm{~mL}, 0.6 \mathrm{mmol}), \mathrm{Pd}(\mathrm{acac})_{2}(2.4 \mathrm{mg}, 0.008 \mathrm{mmol}, 0.04$ equiv), Sphos (4.9 mg, 
$0.012 \mathrm{mmol}, 0.06$ equiv), at $95{ }^{\circ} \mathrm{C}$ for fourteen hours using General Procedure B. The crude material was purified via column chromatography on silica gel using 98:2 -> 95:5 hexanes:ethyl acetate as the eluent. This procedure afforded $40.1 \mathrm{mg}(52 \%)$ of the title compound as a colorless oil. The compound was obtained as a $>20: 1$ mixture of diastereomers as judged by ${ }^{1} \mathrm{H}$ NMR analysis. Data are for the major isomer. ${ }^{1} \mathrm{H}$ NMR $\left(500 \mathrm{MHz}, \mathrm{CDCl}_{3}\right) \delta 5.54(\mathrm{q}, J=3.1 \mathrm{~Hz}$, 1H), $4.17(\mathrm{ddt}, J=11.1,7.6,4.4 \mathrm{~Hz}, 6 \mathrm{H}), 3.39-3.30(\mathrm{~m}, 1 \mathrm{H}), 3.26(\mathrm{~d}, J=6.0 \mathrm{~Hz}, 1 \mathrm{H}), 2.44-$ $2.31(\mathrm{~m}, 2 \mathrm{H}), 2.23(\mathrm{dt}, J=12.5,3.4 \mathrm{~Hz}, 1 \mathrm{H}), 2.18-2.07(\mathrm{~m}, 1 \mathrm{H}), 1.98(\mathrm{ddq}, J=14.6,7.1,3.5$ $\mathrm{Hz}, 2 \mathrm{H}), 1.76-1.59(\mathrm{~m}, 2 \mathrm{H}), 1.53(\mathrm{ddd}, J=13.9,6.9,3.5 \mathrm{~Hz}, 1 \mathrm{H}), 1.26(\mathrm{t}, J=7.1 \mathrm{~Hz}, 9 \mathrm{H}), 1.12$ $(\mathrm{d}, J=6.7 \mathrm{~Hz}, 3 \mathrm{H}) .{ }^{13} \mathrm{C} \operatorname{NMR}\left(126 \mathrm{MHz}, \mathrm{CDCl}_{3}\right) \delta 175.5,169.0,168.4,141.8,114.8,114.4$, 61.3, 61.1 (2 peaks), 60.6, 54.8, 51.0, 49.7, 37.9, 31.7, 24.2, 19.6, 16.3, 14.1, 14.0. IR (film) 1725.2 (2 peaks), $1446.7 \mathrm{~cm}^{-1}$. HRMS (ESI ${ }^{+}$TOF) $\mathrm{m} / \mathrm{z}:\left[\mathrm{M}+\mathrm{H}^{+}\right]^{+}$calcd for $\mathrm{C}_{20} \mathrm{H}_{30} \mathrm{O}_{6} 367.2121$; found 367.2115 .

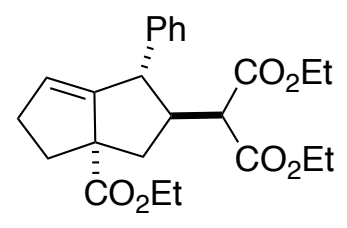

(士)-(1S,2S,3aS)-Diethyl 2-[3a-(Ethoxycarbonyl)-1-phenyl-1,2,3,3a,4,5-hexahydropentalen2-yl] malonate (6k). The title compound was prepared from ethyl 1-cinnamyl-2$\{[$ (trifluoromethyl)sulfonyl]oxy\}cyclopent-2-ene-1-carboxylate $(70.9 \mathrm{mg}, 0.18 \mathrm{mmol})$ and diethyl malonate $(0.1 \mathrm{~mL}, 0.6 \mathrm{mmol}), \mathrm{Pd}(\mathrm{acac})_{2}(2.4 \mathrm{mg}, 0.008 \mathrm{mmol}, 0.04$ equiv), Sphos (4.9 mg, $0.012 \mathrm{mmol}, 0.06$ equiv), at $95{ }^{\circ} \mathrm{C}$ for fourteen hours using General Procedure $\mathrm{B}$. The crude material was purified via column chromatography on silica gel using 95:5 -> 90:10 hexanes:ethyl acetate as the eluent. This procedure afforded $53.8 \mathrm{mg}(72 \%)$ of the title compound as a yellow oil. The compound was obtained as a $>20: 1$ mixture of diastereomers as judged by ${ }^{1} \mathrm{H}$ NMR analysis. Data are for the major isomer. ${ }^{1} \mathrm{H}$ NMR $\left(500 \mathrm{MHz}, \mathrm{CDCl}_{3}\right) \delta 7.32-$ $7.08(\mathrm{~m}, 4 \mathrm{H}), 5.58(\mathrm{dt}, J=3.5,1.7 \mathrm{~Hz}, 1 \mathrm{H}), 4.27-4.01(\mathrm{~m}, 4 \mathrm{H}), 4.01-3.90(\mathrm{~m}, 1 \mathrm{H}), 3.90-$ 
$3.77(\mathrm{~m}, 1 \mathrm{H}), 3.66-3.57(\mathrm{~m}, 1 \mathrm{H}), 3.48(\mathrm{~d}, J=7.3 \mathrm{~Hz}, 1 \mathrm{H}), 3.24$ (ddt, $J=8.8,7.1,1.8 \mathrm{~Hz}, 1 \mathrm{H}$ ), $3.01-2.87(\mathrm{~m}, 1 \mathrm{H}), 2.77(\mathrm{dd}, J=12.1,6.3 \mathrm{~Hz}, 1 \mathrm{H}), 2.52(\mathrm{ddd}, J=15.8,8.7,3.4 \mathrm{~Hz}, 1 \mathrm{H}), 2.33$ (dd, $J=12.6,6.5 \mathrm{~Hz}, 1 \mathrm{H}$ ), $1.93-1.78(\mathrm{~m}, 1 \mathrm{H}), 1.45$ (t, $J=11.7 \mathrm{~Hz}, 1 \mathrm{H}), 1.23$ (ddt, $J=25.1$, 10.9, 7.2 Hz, 7H), $1.15-1.01(\mathrm{~m}, 3 \mathrm{H}) .{ }^{13} \mathrm{C}$ NMR $\left(126 \mathrm{MHz}, \mathrm{CDCl}_{3}\right) \delta$ 175.8, 168.2, 166.6, 153.3, $142.8,128.3,127.7,126.3,125.9,64.9,61.5,61.4,60.7,55.1,54.3,50.2,47.1,41.7,39.5,37.1$, 29.7, 14.1. IR (film) 1725.0 (2 peaks), $1597.5 \mathrm{~cm}^{-1}$. HRMS (ESI TOF) $\mathrm{m} / \mathrm{z}:\left[\mathrm{M}+\mathrm{H}^{+}\right]^{+}$calcd for $\mathrm{C}_{24} \mathrm{H}_{30} \mathrm{O}_{6} 415.2121$; found 415.2115 .

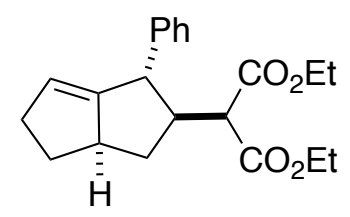

$( \pm)-(1 R, 2 S, 3 \mathrm{a} R)$-Diethyl 2-(1-phenyl-1,2,3,3a,4,5-hexahydropentalen-2-yl)malonate (6I). The title compound was prepared from 5-cinnamylcyclopent-1-en-1-yl trifluoromethanesulfonate (62.4 mg, $0.19 \mathrm{mmol})$ and diethyl malonate $(0.1 \mathrm{~mL}, 0.6 \mathrm{mmol}), \mathrm{Pd}(\mathrm{acac})_{2}(2.4 \mathrm{mg}, 0.008 \mathrm{mmol}$, 0.04 equiv), SPhos ( $4.9 \mathrm{mg}, 0.012 \mathrm{mmol}, 0.06$ equiv), at $95^{\circ} \mathrm{C}$ for fourteen hours using General Procedure B. The crude material was purified via column chromatography on silica gel using 98:2 -> 95:5 hexanes:ethyl acetate as the eluent. This procedure afforded $18.8 \mathrm{mg}$ (30\%) of the title compound as a colorless oil. The compound was obtained as a $>20: 1$ mixture of diastereomers as judged by ${ }^{1} \mathrm{H}$ NMR analysis. Data are for the major isomer. ${ }^{1} \mathrm{H}$ NMR (500 $\left.\mathrm{MHz}, \mathrm{CDCl}_{3}\right) \delta 7.33-7.22(\mathrm{~m}, 3 \mathrm{H}), 7.22-7.10(\mathrm{~m}, 3 \mathrm{H}), 5.25(\mathrm{tt}, J=3.2,1.7 \mathrm{~Hz}, 1 \mathrm{H}), 4.23-$ $4.05(\mathrm{~m}, 2 \mathrm{H}), 3.98-3.84(\mathrm{~m}, 1 \mathrm{H}), 3.84-3.71(\mathrm{~m}, 1 \mathrm{H}), 3.47(\mathrm{dd}, J=21.1,8.3 \mathrm{~Hz}, 2 \mathrm{H}), 3.20(\mathrm{~d}$, $J=6.6 \mathrm{~Hz}, 1 \mathrm{H}), 3.10-2.97(\mathrm{~m}, 1 \mathrm{H}), 2.59(\mathrm{ddd}, J=7.0,3.4,1.7 \mathrm{~Hz}, 1 \mathrm{H}), 2.49(\mathrm{dd}, J=9.7,6.2$ $\mathrm{Hz}, 1 \mathrm{H}), 2.29(\mathrm{dt}, J=12.3,6.4 \mathrm{~Hz}, 1 \mathrm{H}), 2.15(\mathrm{dt}, J=13.2,6.9 \mathrm{~Hz}, 2 \mathrm{H}), 1.24(\mathrm{t}, J=7.1 \mathrm{~Hz}, 3 \mathrm{H})$, $1.05(\mathrm{t}, J=7.1 \mathrm{~Hz}, 3 \mathrm{H}) .{ }^{13} \mathrm{C} \mathrm{NMR}\left(126 \mathrm{MHz}, \mathrm{CDCl}_{3}\right) \delta 168.5,168.4,155.7,144.6,128.3,127.6$, 126.2, 120.2, 61.2, 61.1, 55.7, 52.3, 51.5, 47.2, 37.2, 36.7, 32.1, 14.1, 13.7. IR (film) 2932.7, 
2849.4, 1730.9, 1600.8, 1495.7, $1452.3 \mathrm{~cm}^{-1}$. HRMS (ESI TOF) $\mathrm{m} / \mathrm{z}:\left[\mathrm{M}+\mathrm{H}^{+}\right]^{+}$calcd for $\mathrm{C}_{21} \mathrm{H}_{26} \mathrm{O}_{4} 343.1909$; found 343.1904.

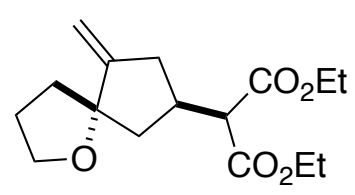

$( \pm)-\left(5 S^{*}, 7 R^{*}\right)$-Diethyl 2-(9-methylene-1-oxaspiro[4.4]nonan-7-yl)malonate $\quad(8)$. The title compound was prepared from 1-(2-allyltetrahydrofuran-2-yl)vinyl triflate $(57.3 \mathrm{mg}, 0.2 \mathrm{mmol})$ and diethyl malonate $(37 \mu \mathrm{L}, 0.24 \mathrm{mmol})$ using General Procedure A. The crude material was purified via column chromatography on silica gel using 95:5 -> 90:10 hexanes:ethyl acetate as the eluent. This procedure afforded $49.0 \mathrm{mg}(82 \%)$ of the title compound as a colorless oil. The compound was obtained as a $>20: 1$ mixture of diastereomers as judged by ${ }^{1} \mathrm{H}$ NMR analysis. Data are for the major isomer. ${ }^{1} \mathrm{H}$ NMR $\left(500 \mathrm{MHz}, \mathrm{C}_{6} \mathrm{D}_{6}\right) \delta 4.96(\mathrm{~s}, 1 \mathrm{H}), 4.88(\mathrm{~s}, 1 \mathrm{H}), 4.07-3.84$ (m, 4H), $3.75-3.59(m, 2 H), 3.31-3.18(m, 1 H), 3.23-3.09(m, 1 H), 2.89(d d, J=16.4,7.8$ $\mathrm{Hz}, 1 \mathrm{H}), 2.29$ (dd, $J=12.9,6.5 \mathrm{~Hz}, 1 \mathrm{H}), 2.16(\mathrm{ddt}, J=16.3,9.5,2.6 \mathrm{~Hz}, 1 \mathrm{H}), 1.87-1.76(\mathrm{~m}$, 1H), $1.71-1.52(\mathrm{~m}, 3 \mathrm{H}), 1.46(\mathrm{dd}, J=12.9,10.7 \mathrm{~Hz}, 1 \mathrm{H}), 0.91(\mathrm{t}, J=7.1 \mathrm{~Hz}, 6 \mathrm{H}) .{ }^{13} \mathrm{C} N M R$ $\left(126 \mathrm{MHz}, \mathrm{C}_{6} \mathrm{D}_{6}\right) \delta 168.12,168.09,154.1,106.3,88.0,66.3,60.63,60.62,57.0,43.8,36.8$, 35.7, 34.4, 26.0, 13.7; IR (film) 1749, 1728, 1445, $1025 \mathrm{~cm}^{-1}$; HRMS (ESI+ TOF) m/z: [M + H $]^{+}$ calcd for $\mathrm{C}_{16} \mathrm{H}_{24} \mathrm{O}_{5} 297.1624$; found 297.1697.

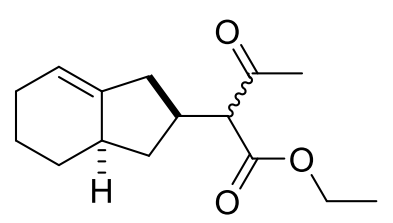

( \pm )-Ethyl $\left(2 S^{*}, 3 \mathrm{a} R^{\star}\right)-2-(2,3,3 \mathrm{a}, 4,5,6-$ hexahydro-1H-inden-2-yl)-3-oxobutanoate (9a). The title compound was prepared from 6-allylcyclohex-1-en-1-yl triflate $(54.1 \mathrm{mg}, 0.2 \mathrm{mmol})$ and ethyl acetoacetate $(31 \mu \mathrm{L}, 0.24 \mathrm{mmol})$ using the General Procedure A. The crude material was purified via column chromatography on silica gel using 99:1 $>$ 95:5 hexanes:ethyl acetate as 
the eluent. This procedure afforded $40.5 \mathrm{mg}(81 \%)$ of the title compound. The compound was obtained as a 1:1 mixture of diastereomers, epimeric at the stereocenter between the carbonyl groups as indicated by the structure above, as judged by ${ }^{1} \mathrm{H}$ NMR analysis. Data are for the mixture. ${ }^{1} \mathrm{H}$ NMR (401 MHz, Chloroform-d) $\delta 5.35(\mathrm{~s}, 0.5 \mathrm{H}), 5.32(\mathrm{~s}, 0.5 \mathrm{H}), 4.28-4.10(\mathrm{~m}, 2 \mathrm{H})$, $3.25(\mathrm{dd}, J=10.1,8.2 \mathrm{~Hz}, 1 \mathrm{H}), 2.75-2.50(\mathrm{~m}, 2 \mathrm{H}), 2.22(\mathrm{~d}, J=3.1 \mathrm{~Hz}, 4 \mathrm{H}), 2.10-1.71(\mathrm{~m}$, $6 \mathrm{H}), 1.51-1.34(\mathrm{~m}, 1 \mathrm{H}), 1.29-1.25(\mathrm{~m}, 3 \mathrm{H}), 1.05-0.89(\mathrm{~m}, 1 \mathrm{H}), 0.88-0.71(\mathrm{~m}, 1 \mathrm{H}) ;{ }^{13} \mathrm{C}$ NMR $\left(126 \mathrm{MHz}_{\mathrm{CDCl}}\right) \delta$ 203.1, 202.9, 169.33, 169.28, 142.7, 142.5, 118.3, 118.2, 66.4, 66.2, $61.4,41.1,40.8,38.6,38.59,36.9,35.2,35.2,29.3,29.0,28.9,25.30,25.29,22.5,22.4,14.3$; IR (film) 1735, $1710 \mathrm{~cm}^{-1}$; HRMS (ESI TOF) m/z: [M + Na $]^{+}$calcd for $\mathrm{C}_{15} \mathrm{H}_{22} \mathrm{O}_{3}$ 273.1569; found 273.1461.

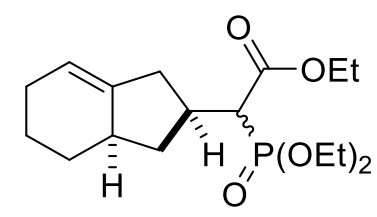

$( \pm)-\left(2 S^{*}, 3 a R^{*}\right)-E t h y l$

2-(diethoxyphosphoryl)-2-(2,3,3a,4,5,6-hexahydro-1 H-inden-2-

yl)acetate (9b). The title compound was prepared from 6-allylcyclohex-1-en-1-yl triflate (500 $\mathrm{mg}, 1.85 \mathrm{mmol})$, and triethyl phosphonoacetate $(440 \mu \mathrm{L}, 2.22 \mathrm{mmol})$ using General Procedure A except with lithium hexamethyldisilazide $(774 \mathrm{mg}, 4.625 \mathrm{mmol})$ as base. The crude material was purified via column chromatography on silica gel using 50:50 -> 15:85 hexanes:ethyl acetate as the eluent. This procedure afforded $332 \mathrm{mg}(52 \%)$ of the title compound as a yellow oil. The compound was obtained as a 1:1 mixture of diastereomers, epimeric at the stereocenter adjacent to the ester group as indicated by the structure above, as judged by ${ }^{1} \mathrm{H}$ NMR analysis. Data are for the mixture ${ }^{1} \mathrm{H}$ NMR $\left(401 \mathrm{MHz}^{\mathrm{CDCl}}{ }_{3}\right) \delta 5.39(\mathrm{~s}, 0.5 \mathrm{H}), 5.35(\mathrm{~s}, 0.5 \mathrm{H}), 4.35-3.98$ $(\mathrm{m}, 6 \mathrm{H}), 2.84-2.54(\mathrm{~m}, 2.5 \mathrm{H}), 2.35-2.09(\mathrm{~m}, 2 \mathrm{H}), 2.07-1.86(\mathrm{~m}, 4 \mathrm{H}), 1.85-1.71(\mathrm{~m}, 1 \mathrm{H})$, $1.50-1.37(\mathrm{~m}, 1 \mathrm{H}), 1.41-1.14(\mathrm{~m}, 9 \mathrm{H}), 1.14-0.73(\mathrm{~m}, 2.5 \mathrm{H}) ;{ }^{13} \mathrm{C}$ NMR $\left(126 \mathrm{MHz}, \mathrm{CDCl}_{3}\right) \delta$ $169.21,169.06,142.83,142.20,117.97,62.46,61.21,52.14,51.09,41.32,40.64,39.63,39.51$, 
$39.42,36.28,36.24,36.15,36.11,35.92,35.79,35.43,28.65,28.58,25.19,25.14,22.32,22.30$, 16.40, 16.36, 14.17; IR (film) $1731 \mathrm{~cm}^{-1}$; HRMS (ESI+ TOF) m/z: $\left[\mathrm{M}+\mathrm{H}^{+}\right]^{+}$calcd for $\mathrm{C}_{17} \mathrm{H}_{29} \mathrm{O}_{5} \mathrm{P}$ 345.1753 ; found 345.1825 .

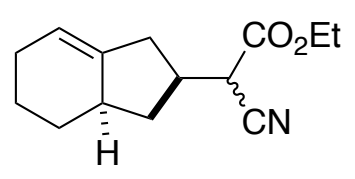

(士)-(2S, 2'R, 3aR)-Ethyl 2'-cyano-2-2,3,3a,4,5,6-hexahydro-1H-inden-2-yl acetate (9c). The title compound was prepared from 6-allylcyclohex-1-en-1-yl triflate $(54.1 \mathrm{mg}, 0.2 \mathrm{mmol})$, ethyl cyanoacetate $(26 \mu \mathrm{L}, 0.24 \mathrm{mmol})$, using General Procedure A, except with $\mathrm{Pd}($ allyl)BrettphosCl (8.6 $\mathrm{mg}, 0.012 \mathrm{mmol}$ ), as catalyst, BrettPhos was added (6.4 mg, $0.012 \mathrm{mmol})$, cesium carbonate $(91.2 \mathrm{mg}, 0.28 \mathrm{mmol})$ as base, and 1,4 dioxane $(2 \mathrm{~mL}, 0.1 \mathrm{M})$ as solvent. The crude material was purified via column chromatography on silica gel using 65:35 -> 50:50 hexanes:dichloromethane as the eluent. This procedure afforded $22.8 \mathrm{mg}(50 \%)$ of the title compound as a colorless oil. The compound was obtained as a 1:1 mixture of diastereomers, epimeric at the $\mathrm{CN}$-bearing stereocenter, as judged by ${ }^{1} \mathrm{H}$ NMR analysis. Data are for the mixture. ${ }^{1} \mathrm{H}$ NMR $\left(401 \mathrm{MHz}, \mathrm{CDCl}_{3}\right) \delta 5.43(\mathrm{~s}, 1 \mathrm{H}), 4.26(\mathrm{q}, J=7.1 \mathrm{~Hz}, 2 \mathrm{H}), 3.48(\mathrm{dd}, J=11.3$, $6.5 \mathrm{~Hz}, 1 \mathrm{H}), 2.69-2.50(\mathrm{~m}, 2 \mathrm{H}), 2.35-1.91(\mathrm{~m}, 6 \mathrm{H}), 1.85-1.71(\mathrm{~m}, 1 \mathrm{H}), 1.51-1.34(\mathrm{~m}, 1 \mathrm{H})$, $1.32(\mathrm{t}, J=7.1 \mathrm{~Hz}, 3 \mathrm{H}), 1.16-0.97(\mathrm{~m}, 2 \mathrm{H}) ;{ }^{13} \mathrm{C} \mathrm{NMR}\left(126 \mathrm{MHz}, \mathrm{CDCl}_{3}\right) \delta$ 166.0, 141.3, 141.1, 119.32, 119.27, 115.9, 62.8, 42.70, 42.65, 41.1, 41.0, 38.5, 37.9, 37.7, 34.8, 34.0, 28.7, 25.2, 22.3, 14.2; IR (film) 2248, 1743, $1453 \mathrm{~cm}^{-1}$; HRMS (ESI ${ }^{+}$TOF) $\mathrm{m} / \mathrm{z}:\left[\mathrm{M}+\mathrm{H}^{+}\right]^{+}$calcd for $\mathrm{C}_{14} \mathrm{H}_{19} \mathrm{NO}_{2} 234.1416$; found 234.1493 .

\section{Optimization Studies for Reactions of Internal Alkene Substrate 1j}

Table S1. Optimization studies for the coupling of $1 \mathbf{j}$ and diethyl malonate ${ }^{a}$ 


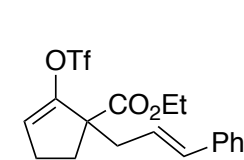

$0.2 \mathrm{mmol}$

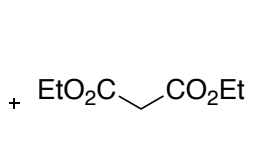

2 eq
$[\mathrm{Pd}](4 \mathrm{~mol} \%)$

Ligand $(6 \mathrm{~mol} \%)$

base $(2.2 \mathrm{eq})$

$\mathrm{PhCH}_{3}(0.8 \mathrm{M}), 95^{\circ} \mathrm{C}, 14 \mathrm{~h}$

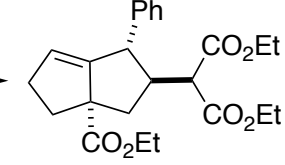

$6 \mathrm{k}$

\begin{tabular}{cccc}
\hline entry & $\mathrm{Pd}$ & ligand & ${\text { yield } \%^{b}(\mathrm{dr})^{c}}^{c}$ \\
\hline 1 & $\mathrm{Pd}(\mathrm{OAc})_{2}$ & BrettPhos & $5(>20: 1)^{e}$ \\
2 & $\mathrm{Pd}(\mathrm{OAc})_{2}$ & CPhos & $47(>20: 1)$ \\
3 & $\mathrm{Pd}(\mathrm{OAc})_{2}$ & RuPhos & $48(>20: 1)$ \\
4 & $\mathrm{Pd}(\mathrm{OAc})_{2}$ & SPhos & $45(>20: 1)$ \\
5 & $\mathrm{Pd}(\mathrm{OAc})_{2}$ & DPEPhos & $65(>20: 1)^{d}$ \\
6 & $\mathrm{Pd}(\mathrm{acac})_{2}$ & DPEPhos & $47(>20: 1)^{e}$ \\
7 & $\mathrm{Pd}(\mathrm{acac})_{2}$ & SPhos & $79(>20: 1)$ \\
\hline
\end{tabular}

aConditions: 1.0 equiv $1 \mathrm{j}, 2.0$ equiv diethyl malonate, $4 \mathrm{~mol} \% \mathrm{Pd}, 6 \mathrm{~mol} \%$ ligand, 2.2 equiv LiOtBu, $0.8 \mathrm{M}$ toluene, $95{ }^{\circ} \mathrm{C}, 14 \mathrm{~h}$. ${ }^{b}$ Isolated yield (average of two or more experiments). ${ }^{c}$ Diastereomeric ratios were determined by ${ }^{1} \mathrm{H}$ NMR analysis. ${ }^{d}$ LiHMDS was used as a base instead of LiOtBu. ${ }^{e}$ The yield in this case was determined by ${ }^{1} \mathrm{H}$ NMR analysis using phenanthrene as an internal standard.

\section{Descriptions of Stereochemical Assignments}

The relative stereochemistry of products $\mathbf{6 d}, \mathbf{6 c}, \mathbf{6 e}, \mathbf{6 k}$, and $\mathbf{6 j}$ was assigned on the basis of ${ }^{1} \mathrm{H}$ NMR nOe studies. The data for these studies is provided below, along with the key nOe signals. The relative stereochemistry of all other products was assigned based on analogy to $\mathbf{6 d}, \mathbf{6 c}, \mathbf{6 e}$, $6 \mathbf{k}$, or $6 \mathbf{j}$. 

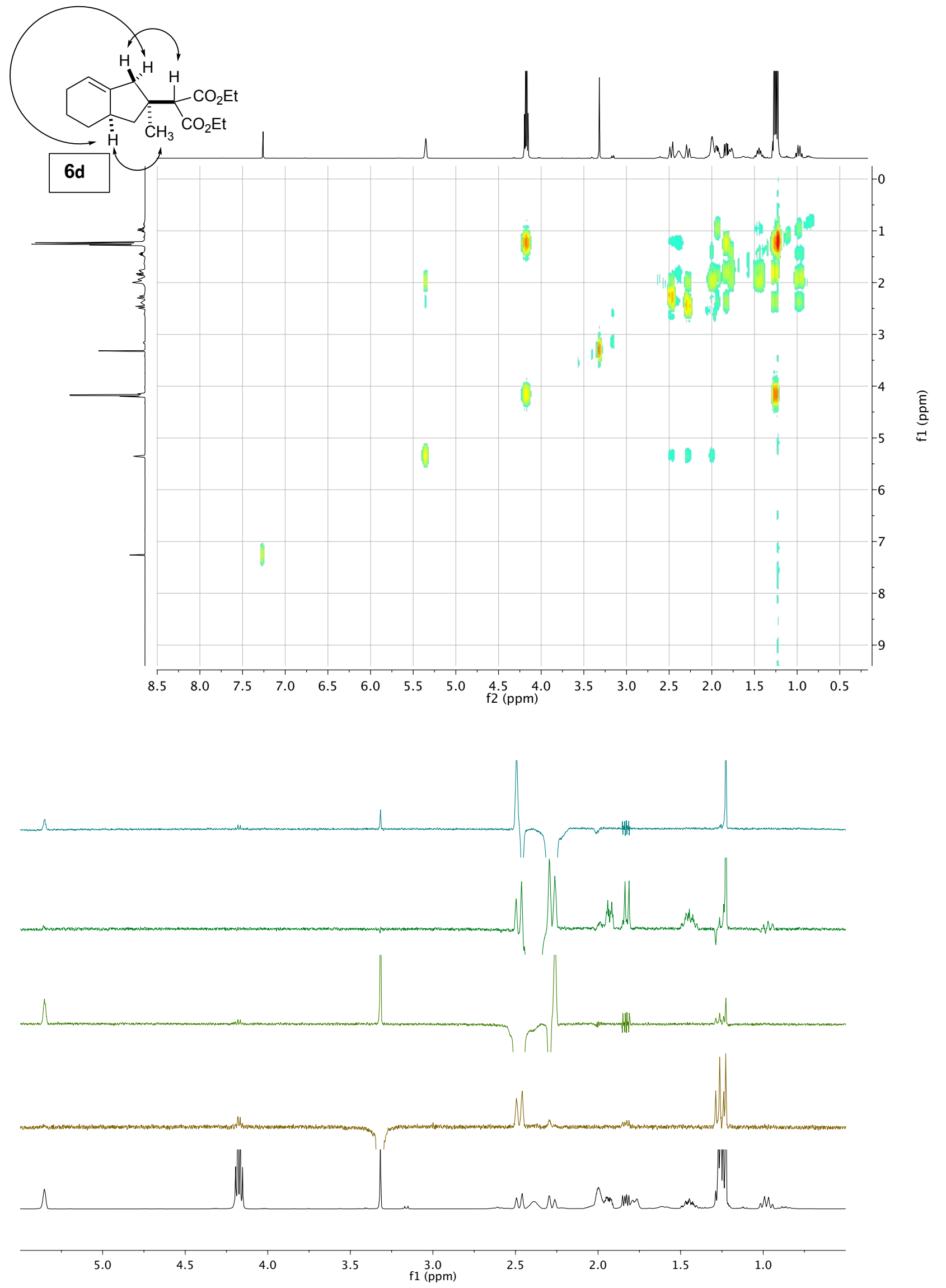

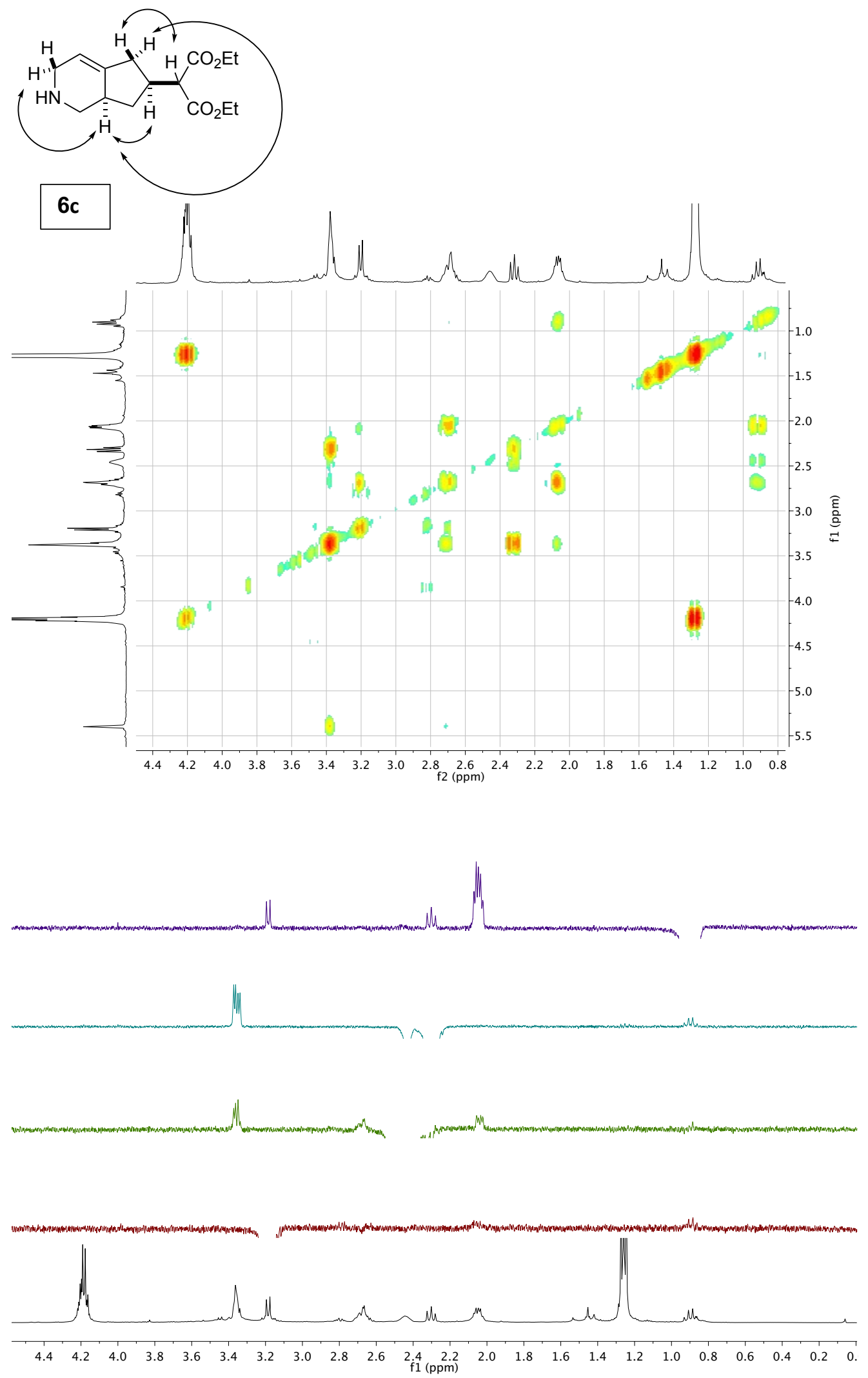

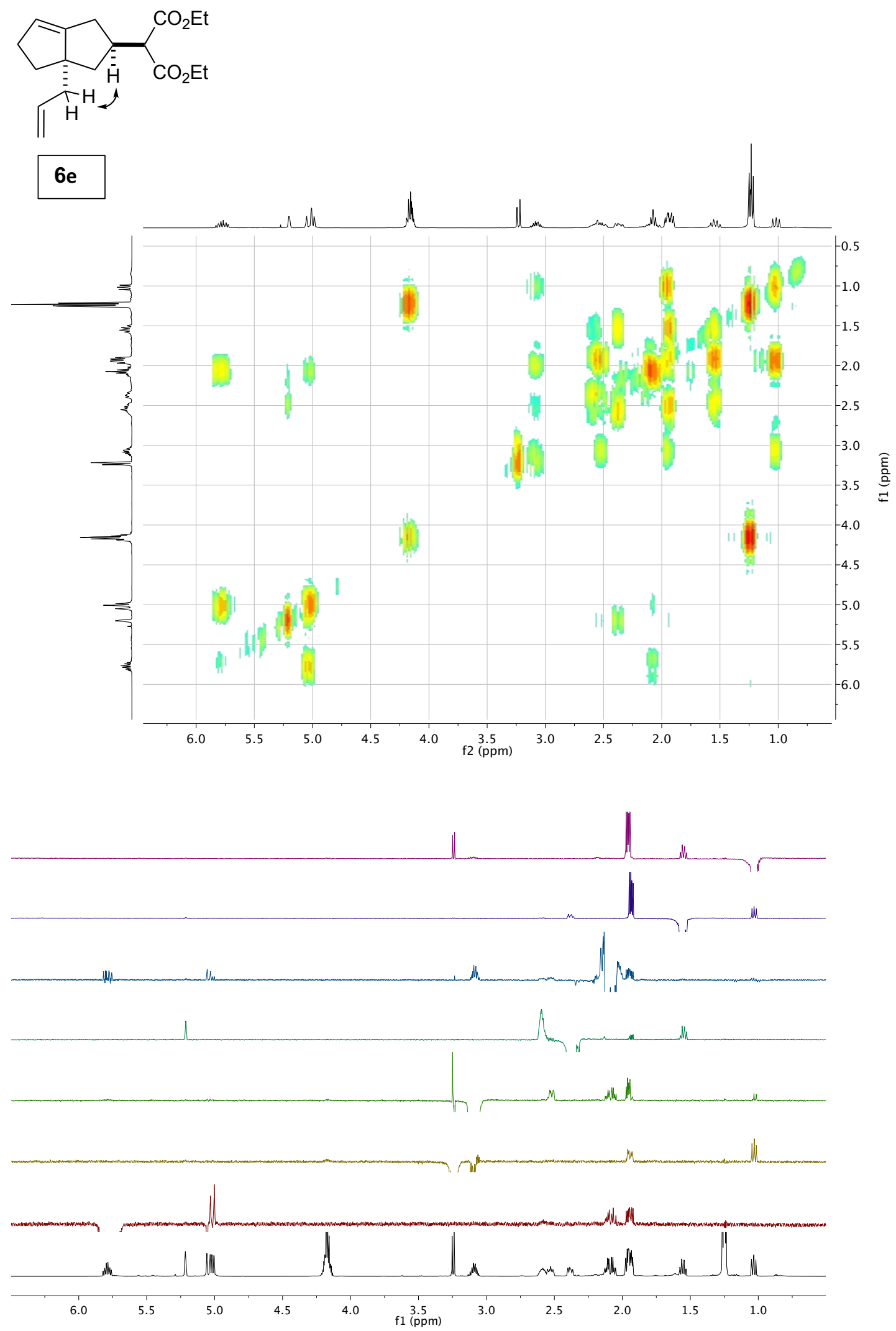

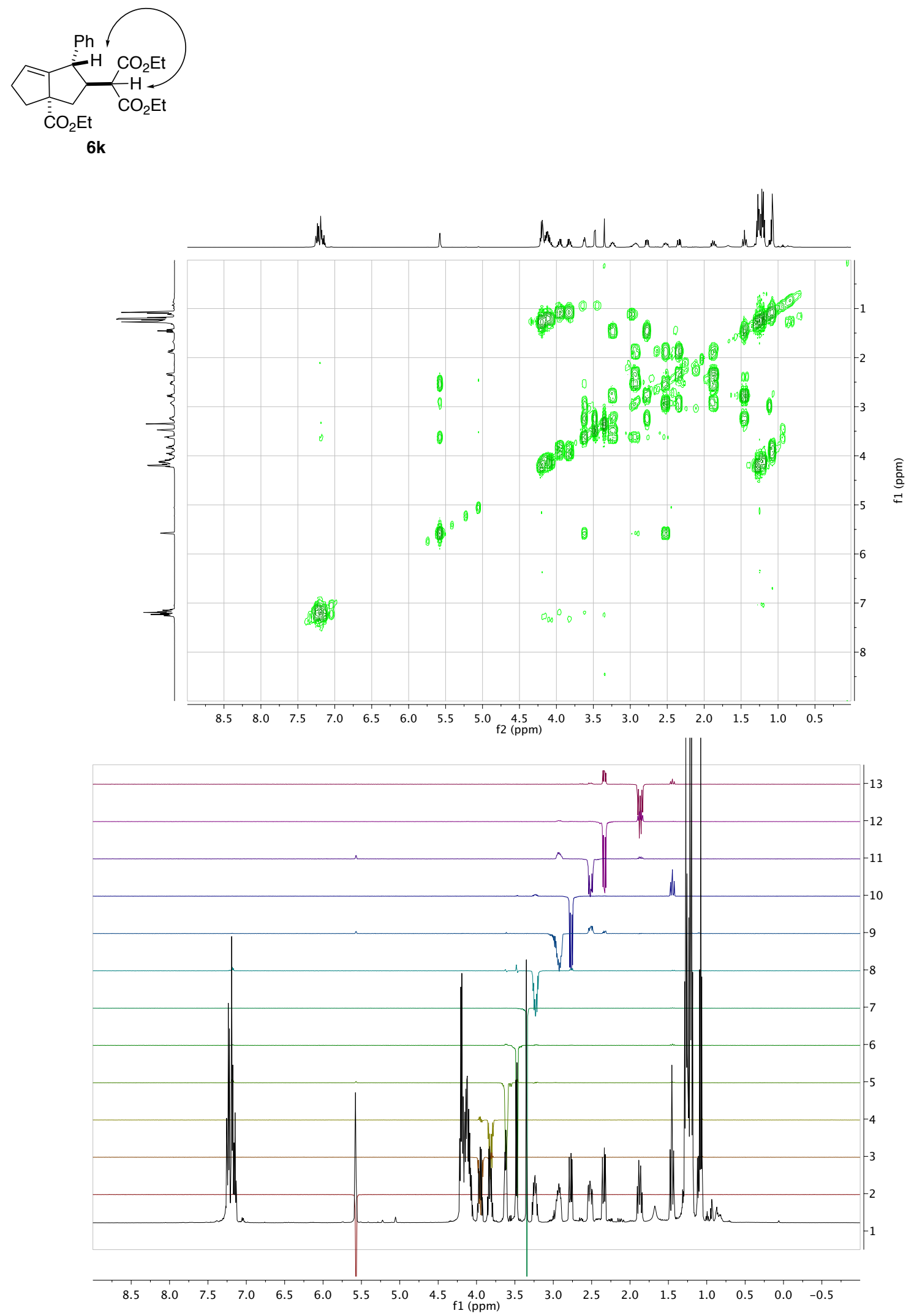


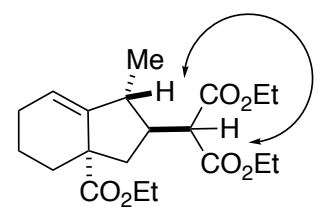

6j
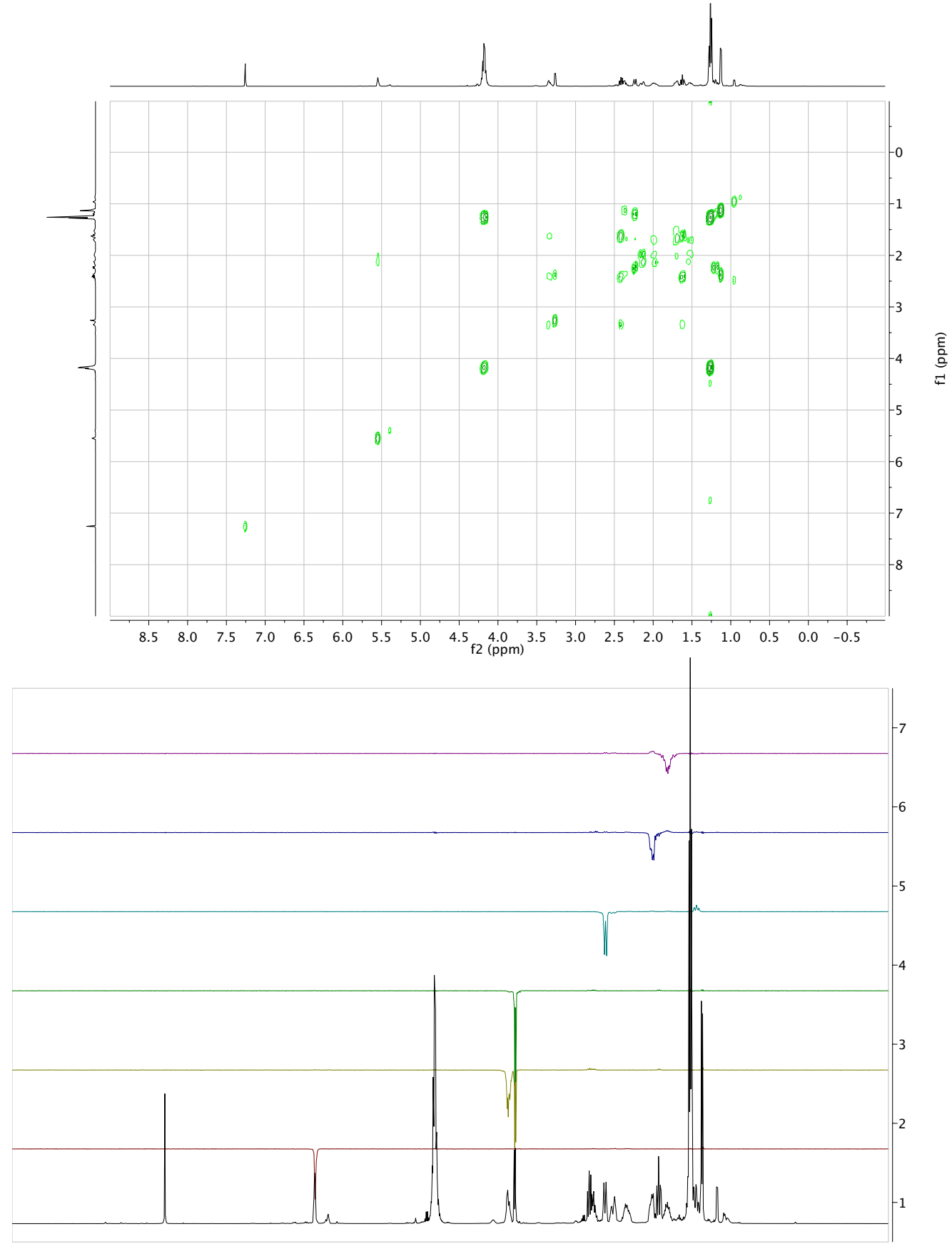

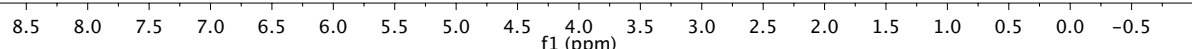



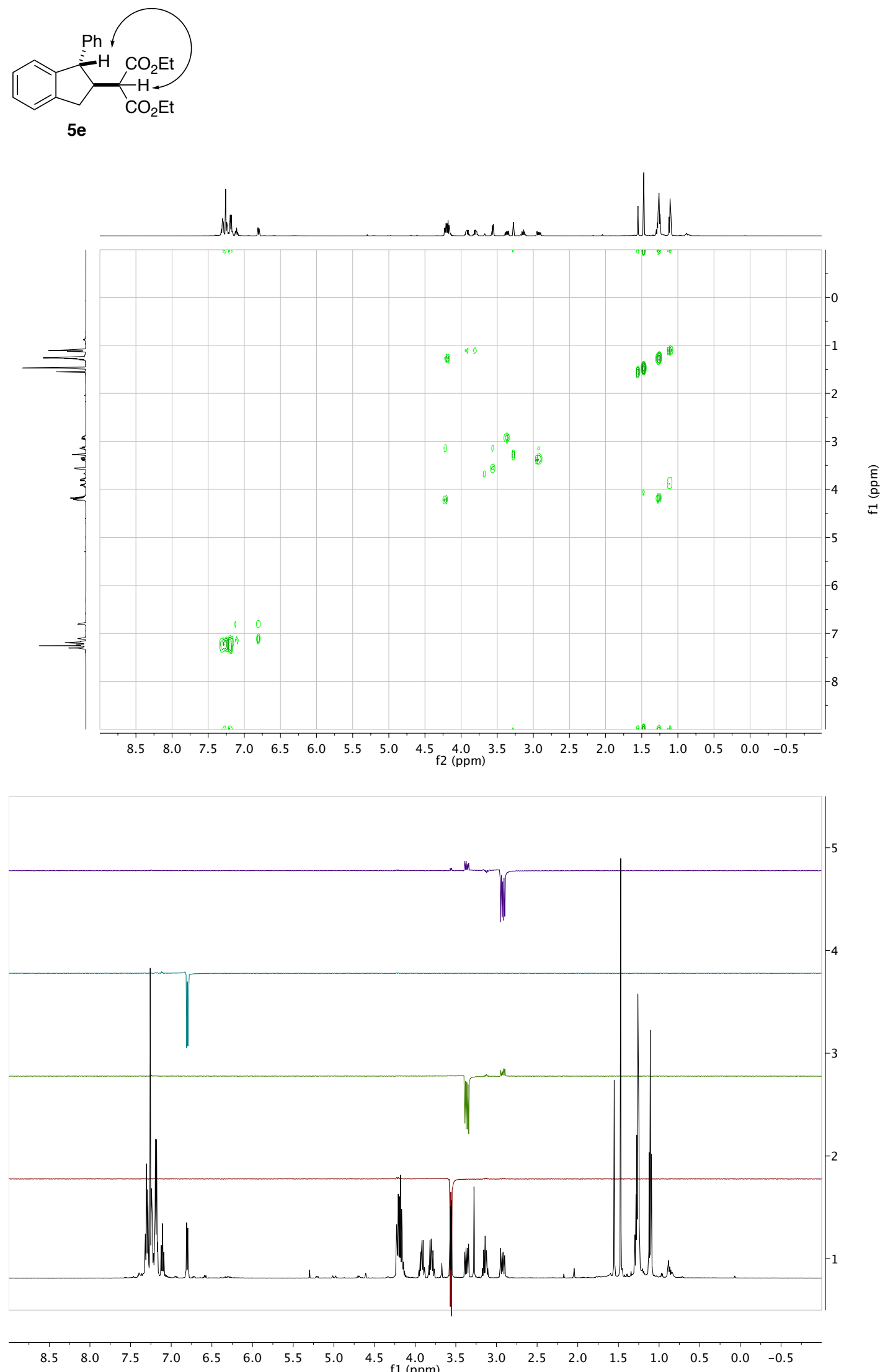


\section{References}

(1) White, D. R.; Wolfe, J. P. Chem. Eur. J. 2017, 19, 4311.

(2) White, D. R.; Herman, M. I.; Wolfe, J. P. Org. Lett. 2017, 19, 4311.

(3) White, D. R.; Hutt, J. T.; Wolfe, J. P. J. Am. Chem. Soc. 2015, 137, 11246.

(4) DeAngelis, A. J.; Gildner, P. G.; Chow, R.; Colacot, T. J. J. Org. Chem. 2015, 80, 6794.

(5) Comins, D. L.; Dehghani, A.; Foti, C. J.; Joseph, S. P. Org. Synth. 1997, 74, 77.

(6) Ramachary, D. B.; Narayana, V. V.; Ramakumar, K. Eur. J. Org. Chem. 2008, 23, 3907. 


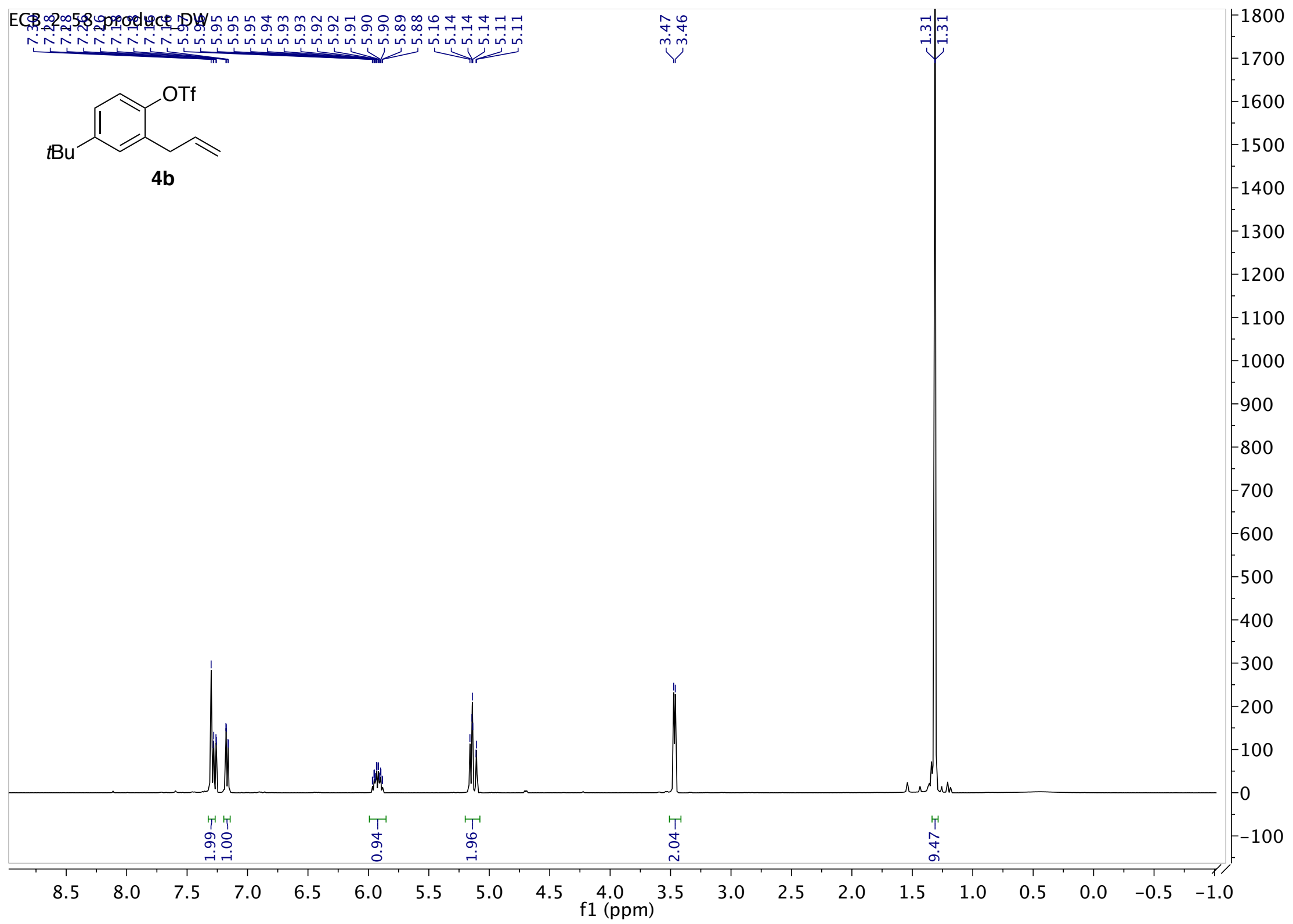




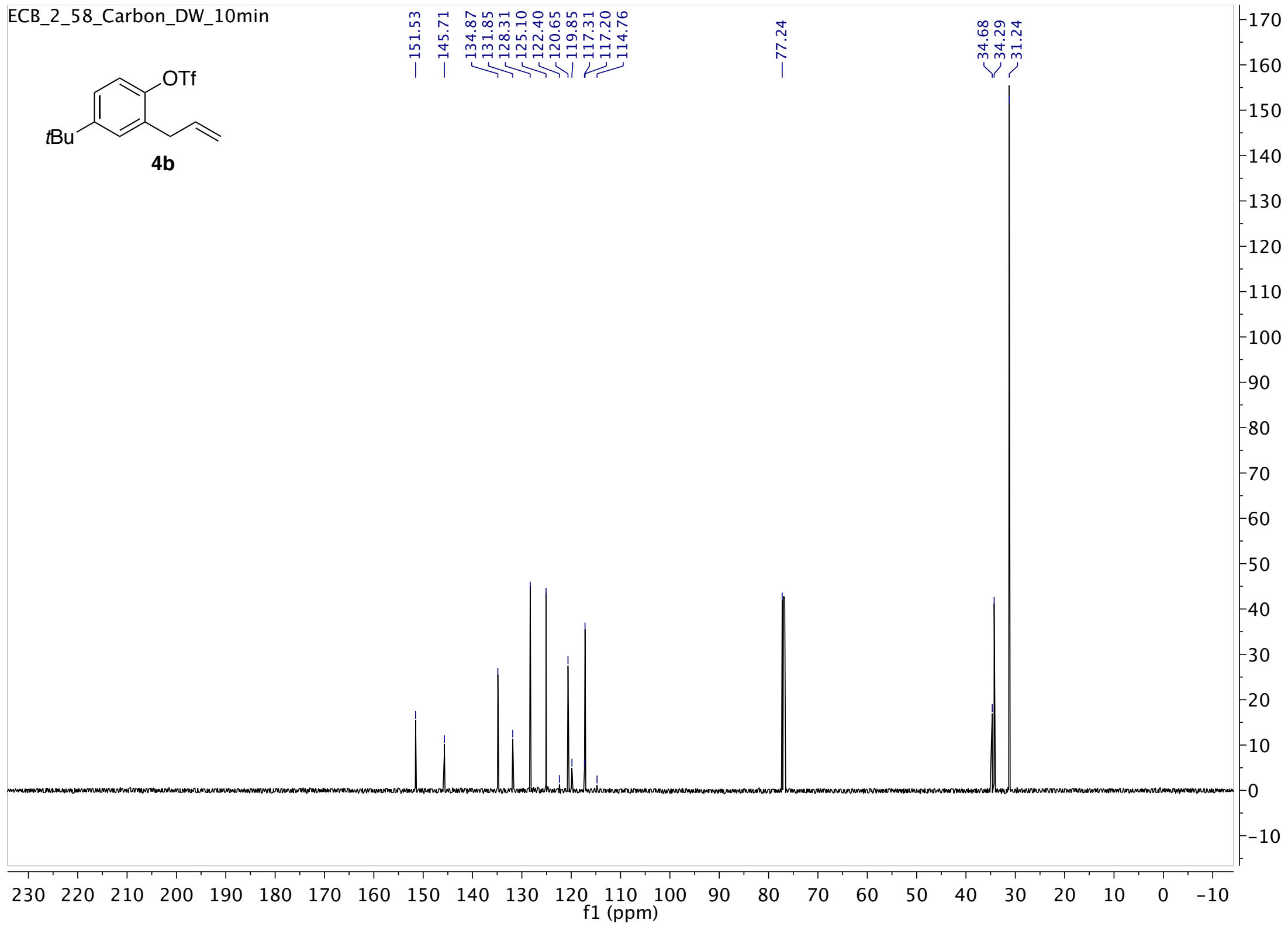




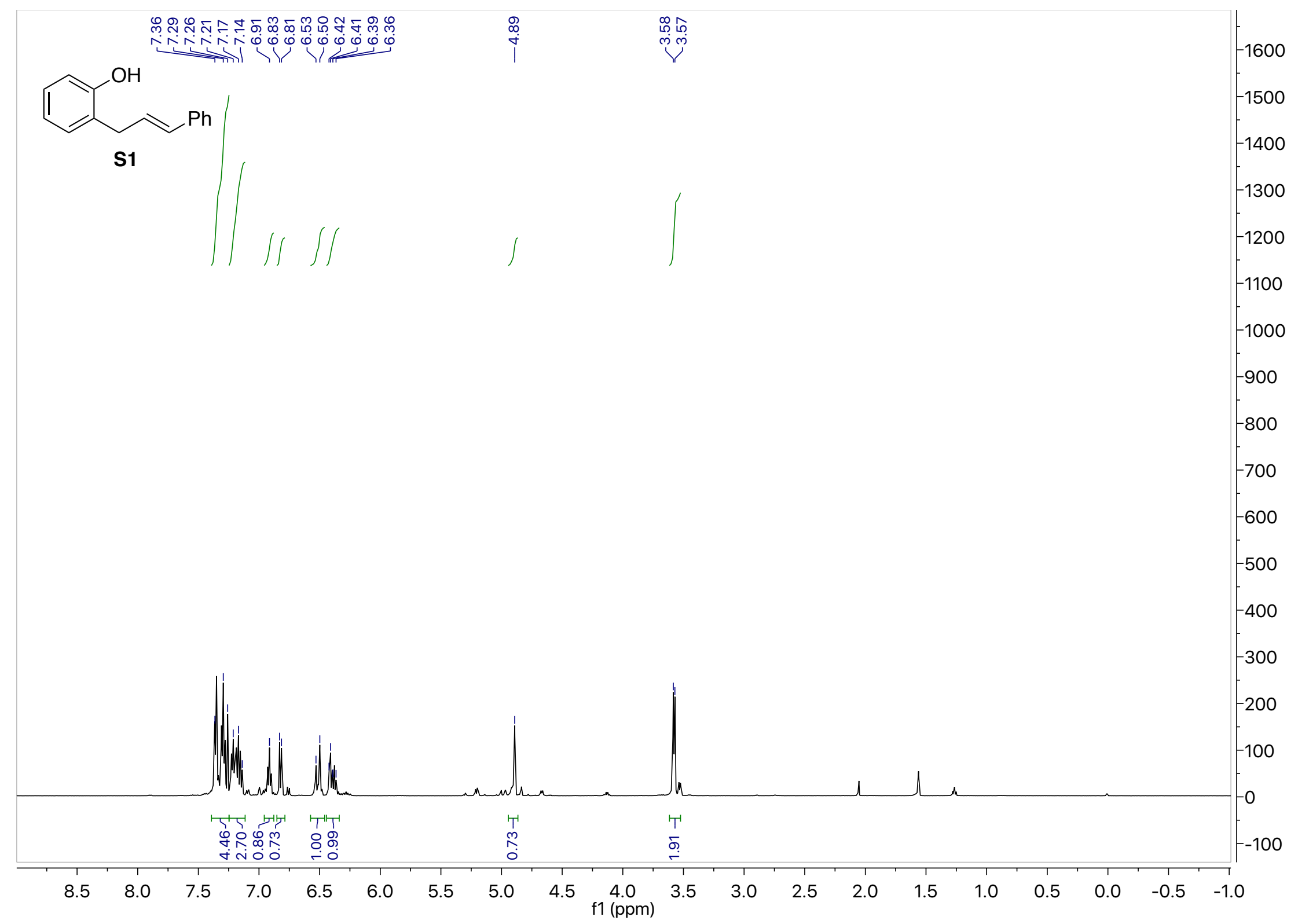




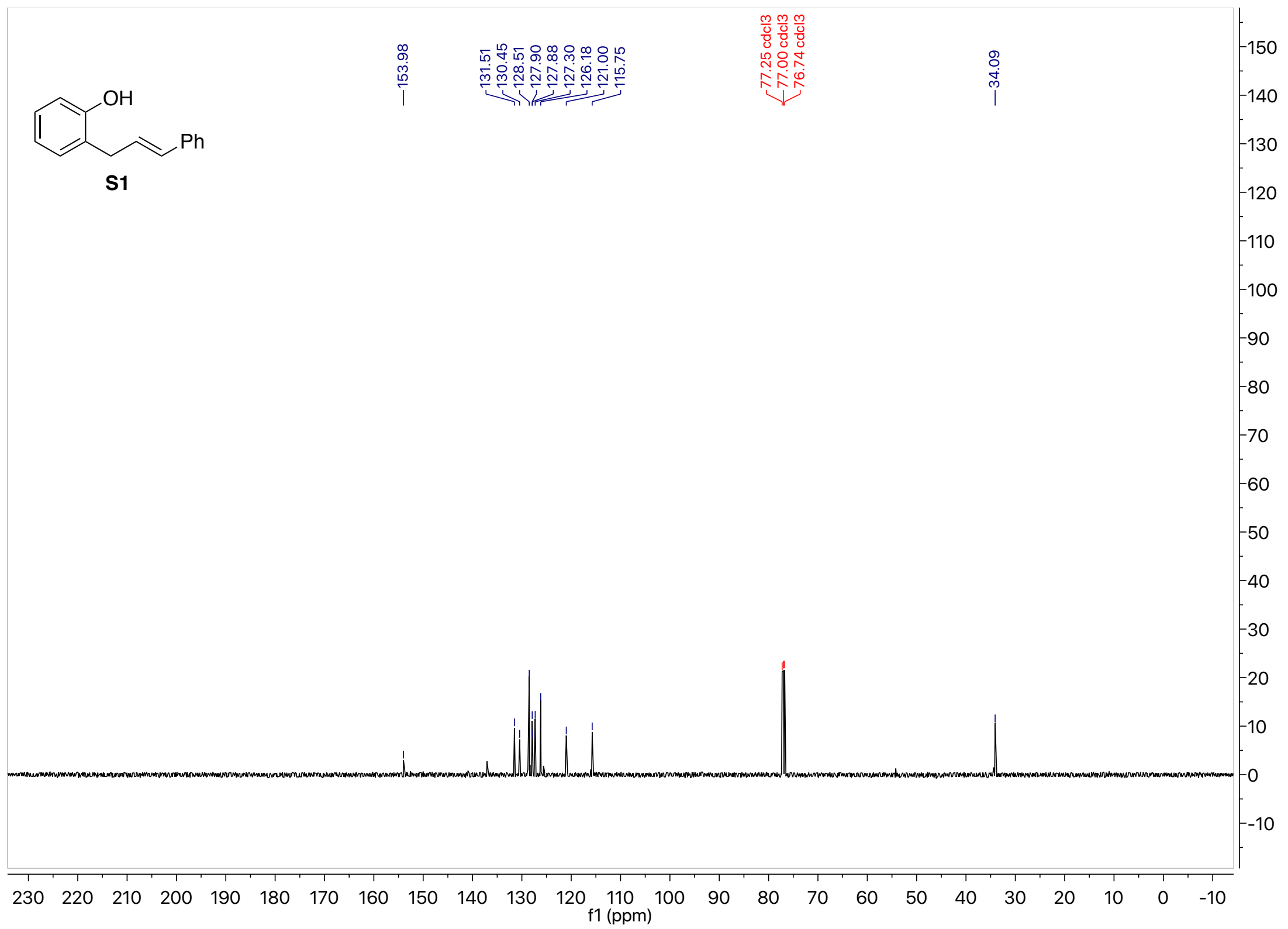




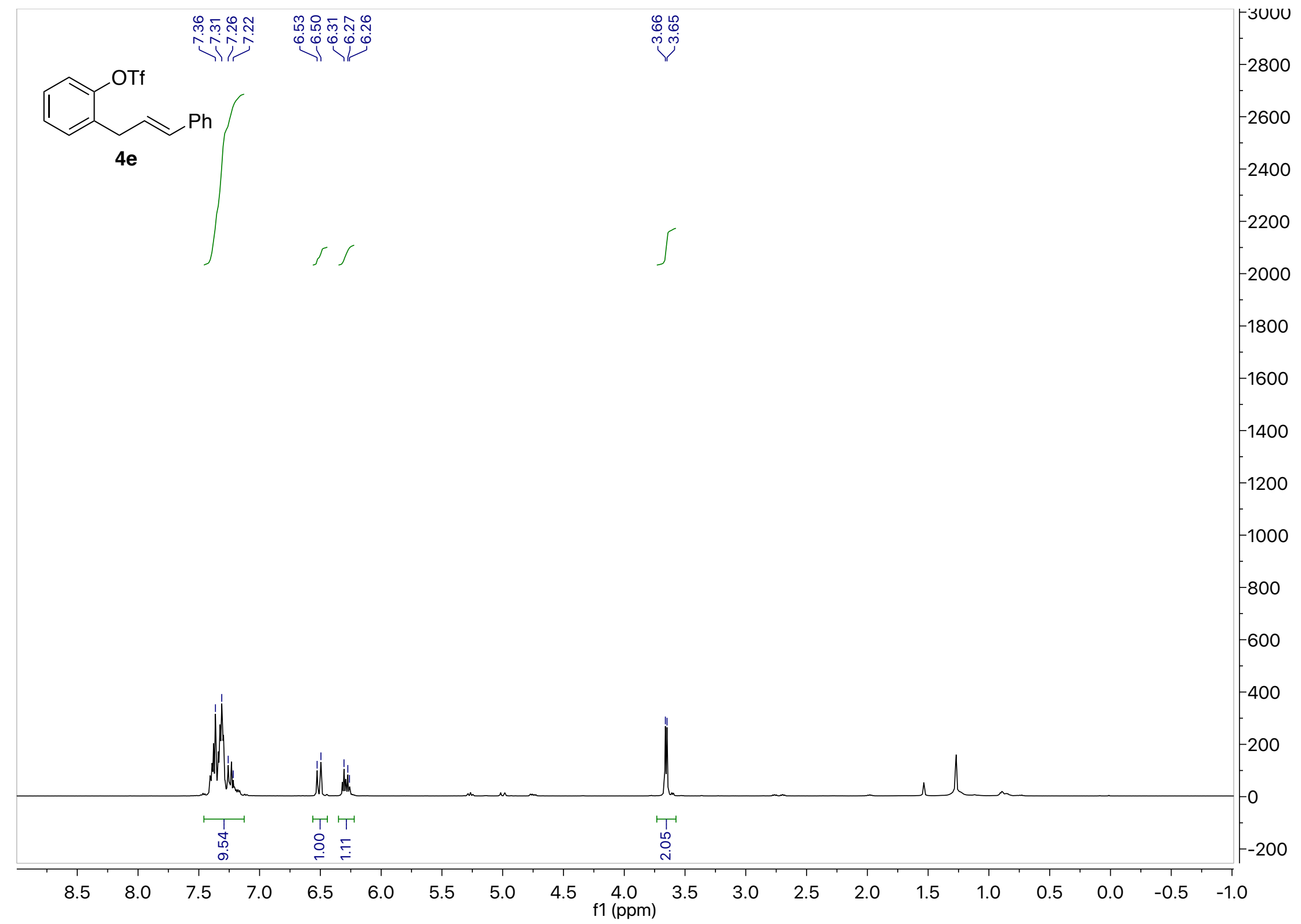




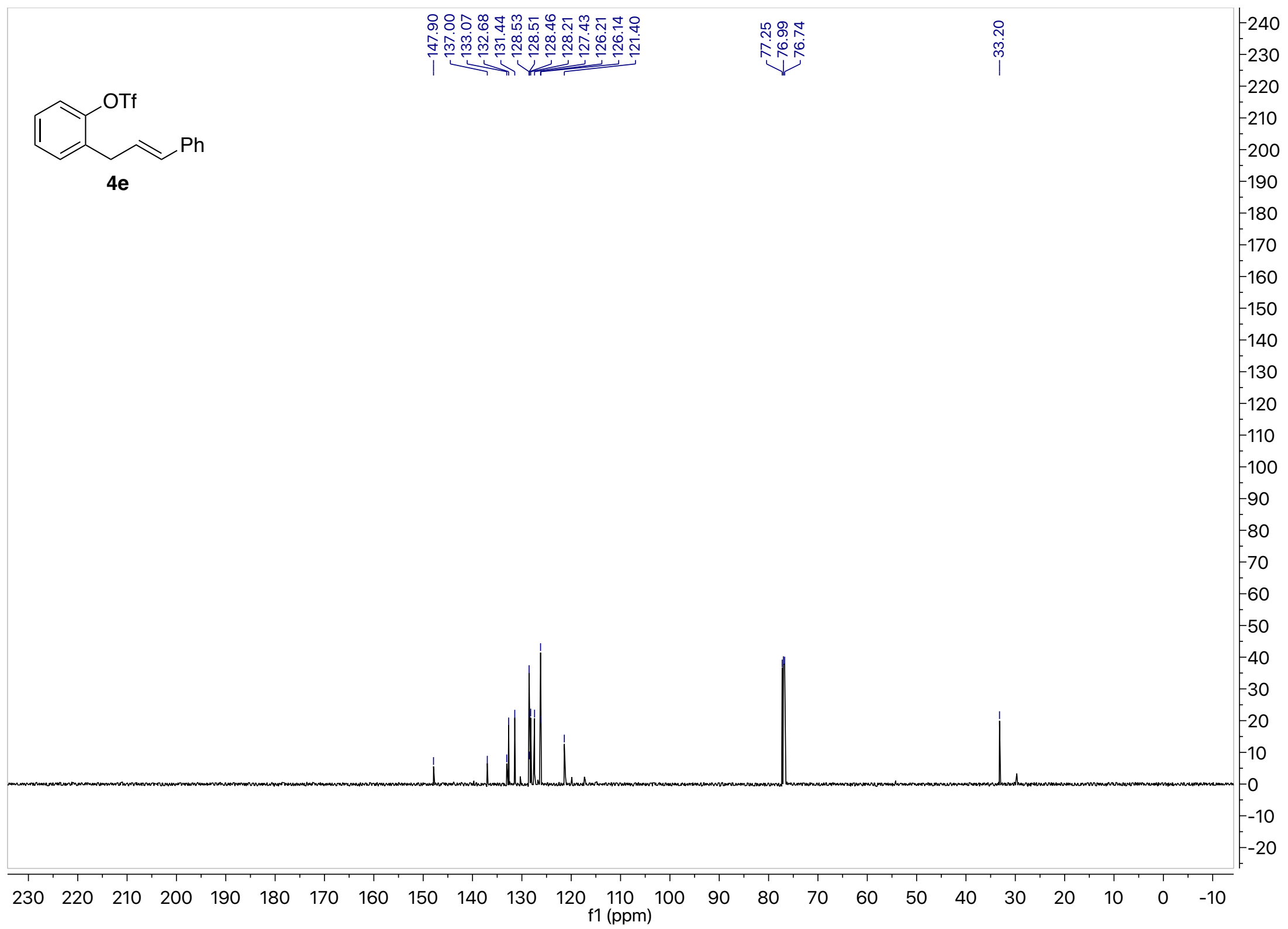




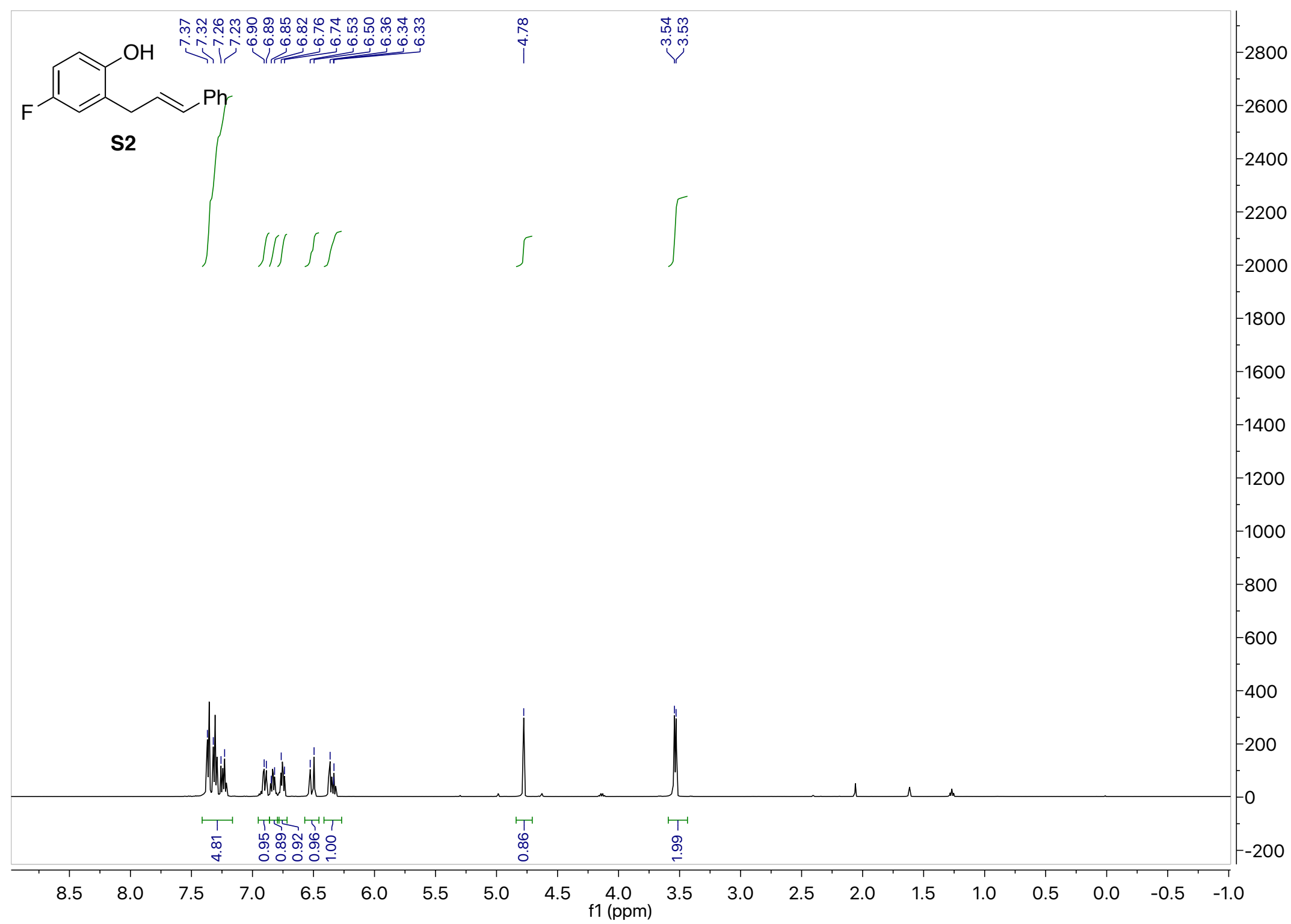




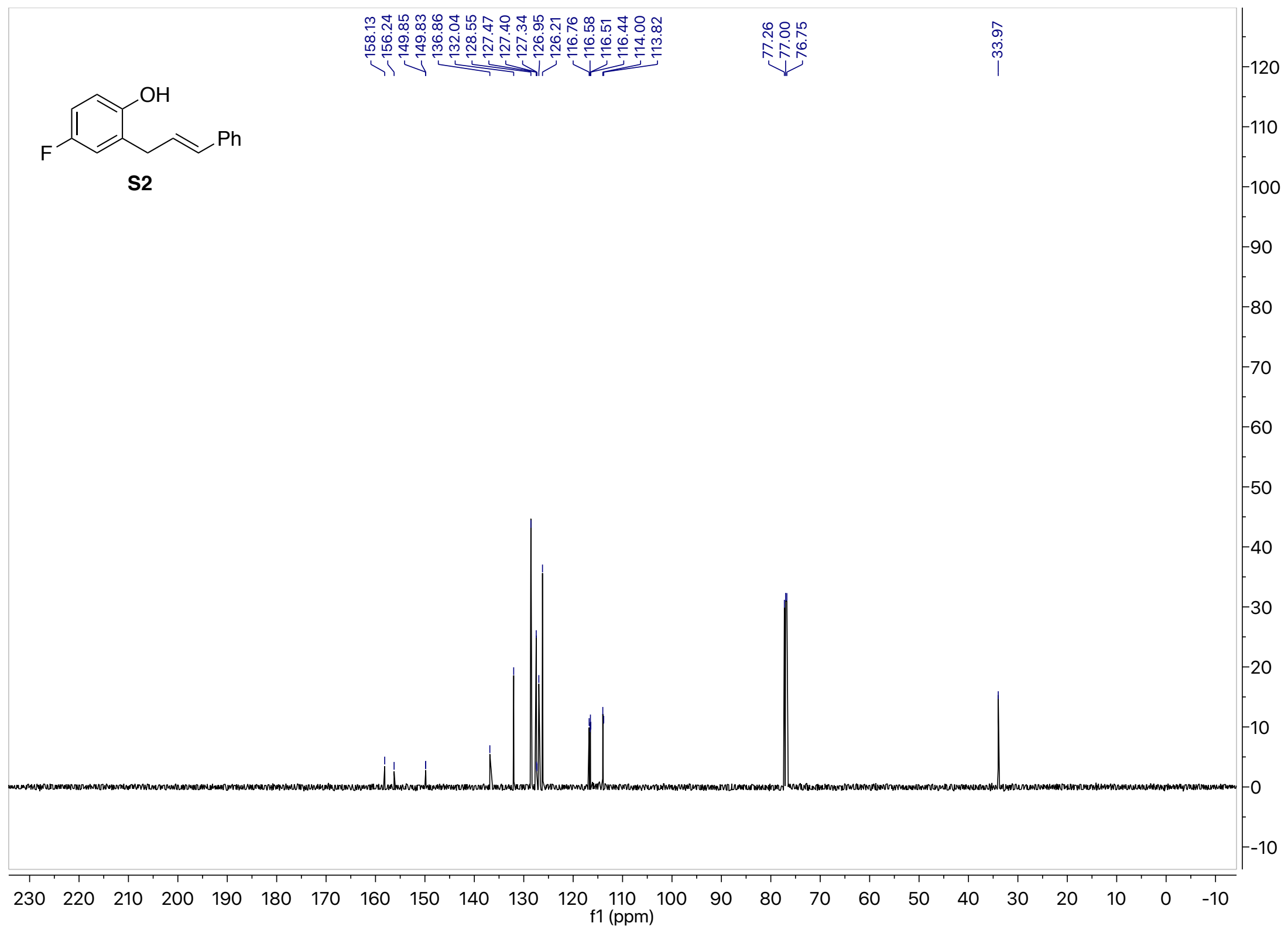




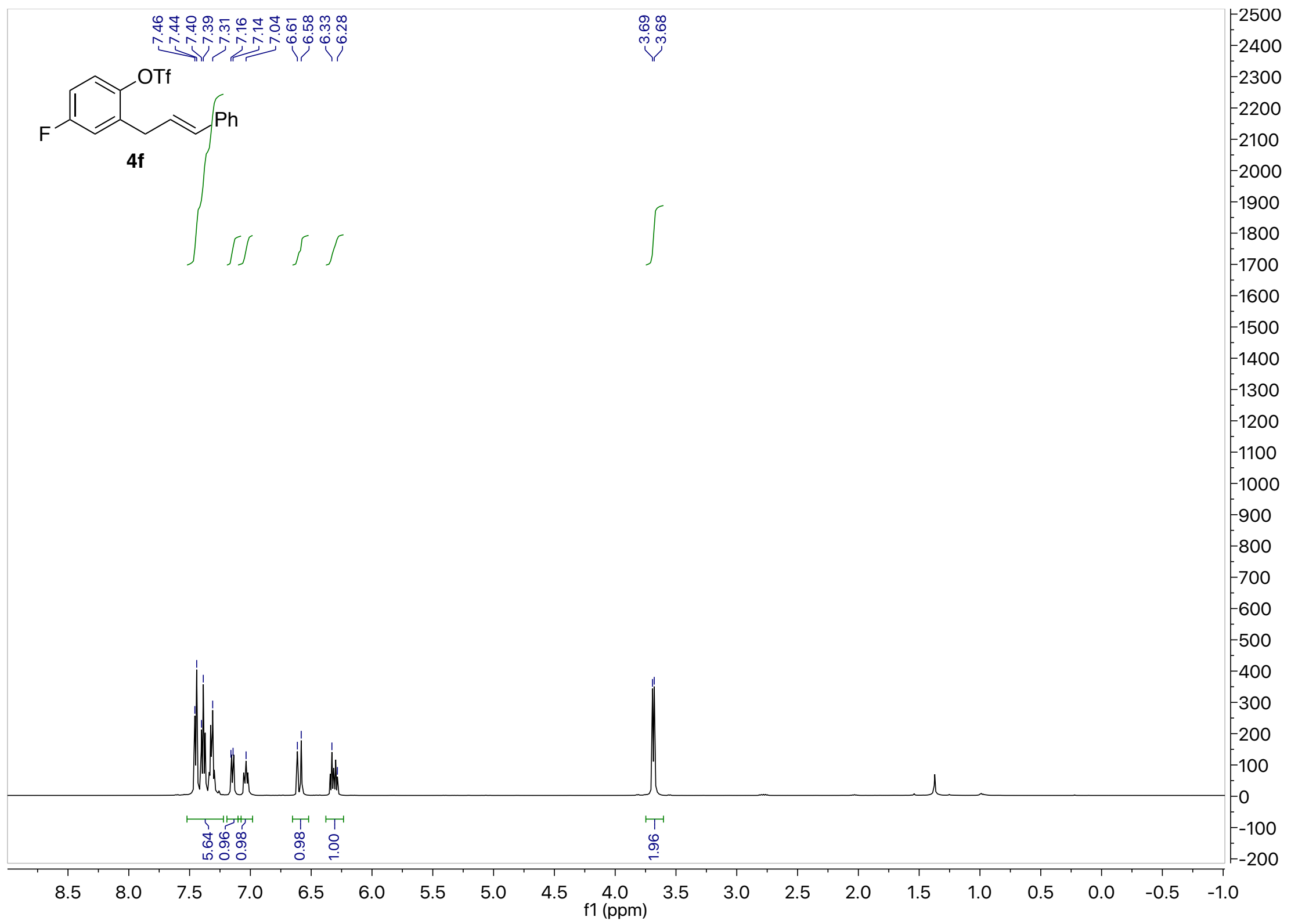




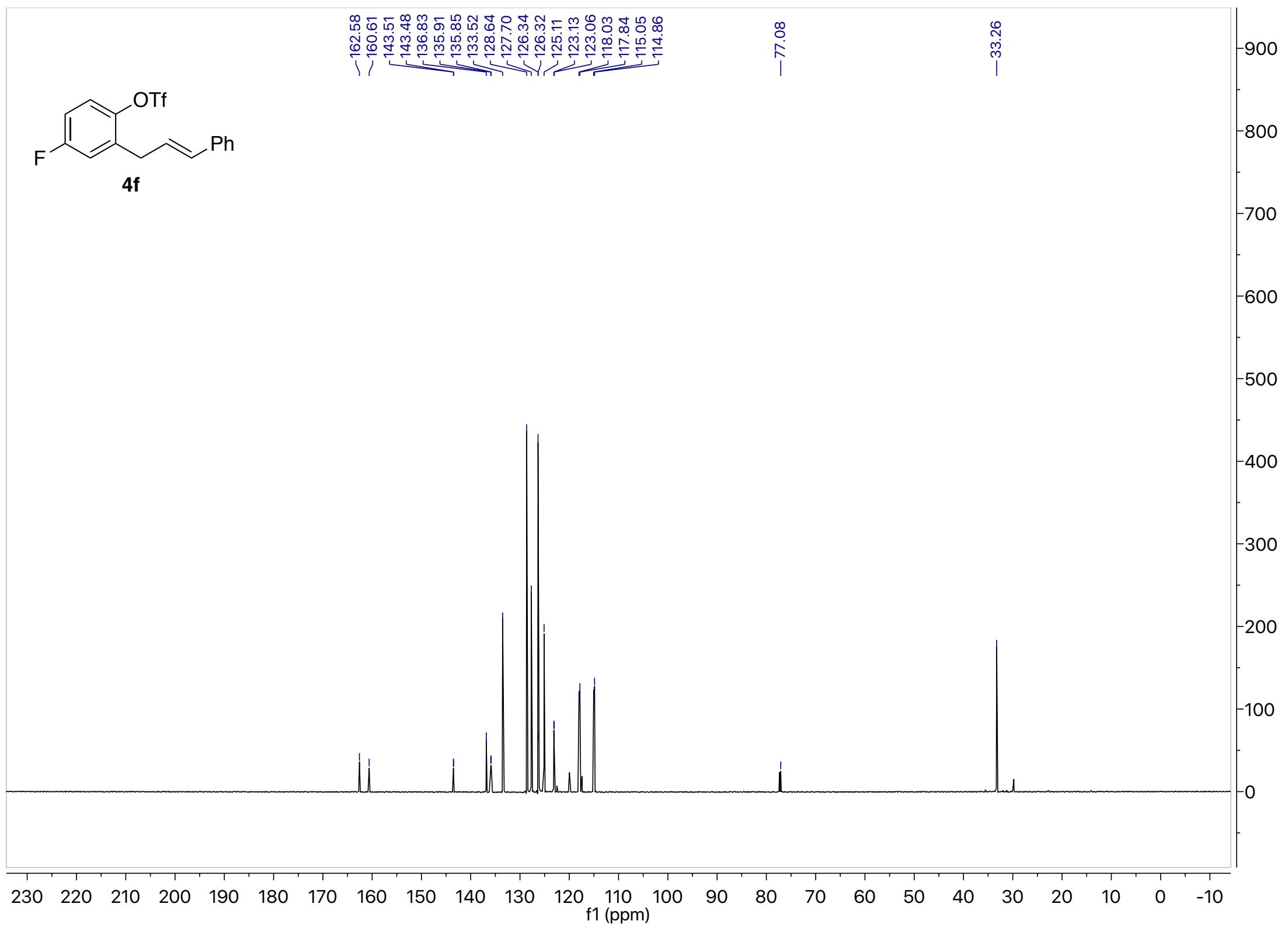




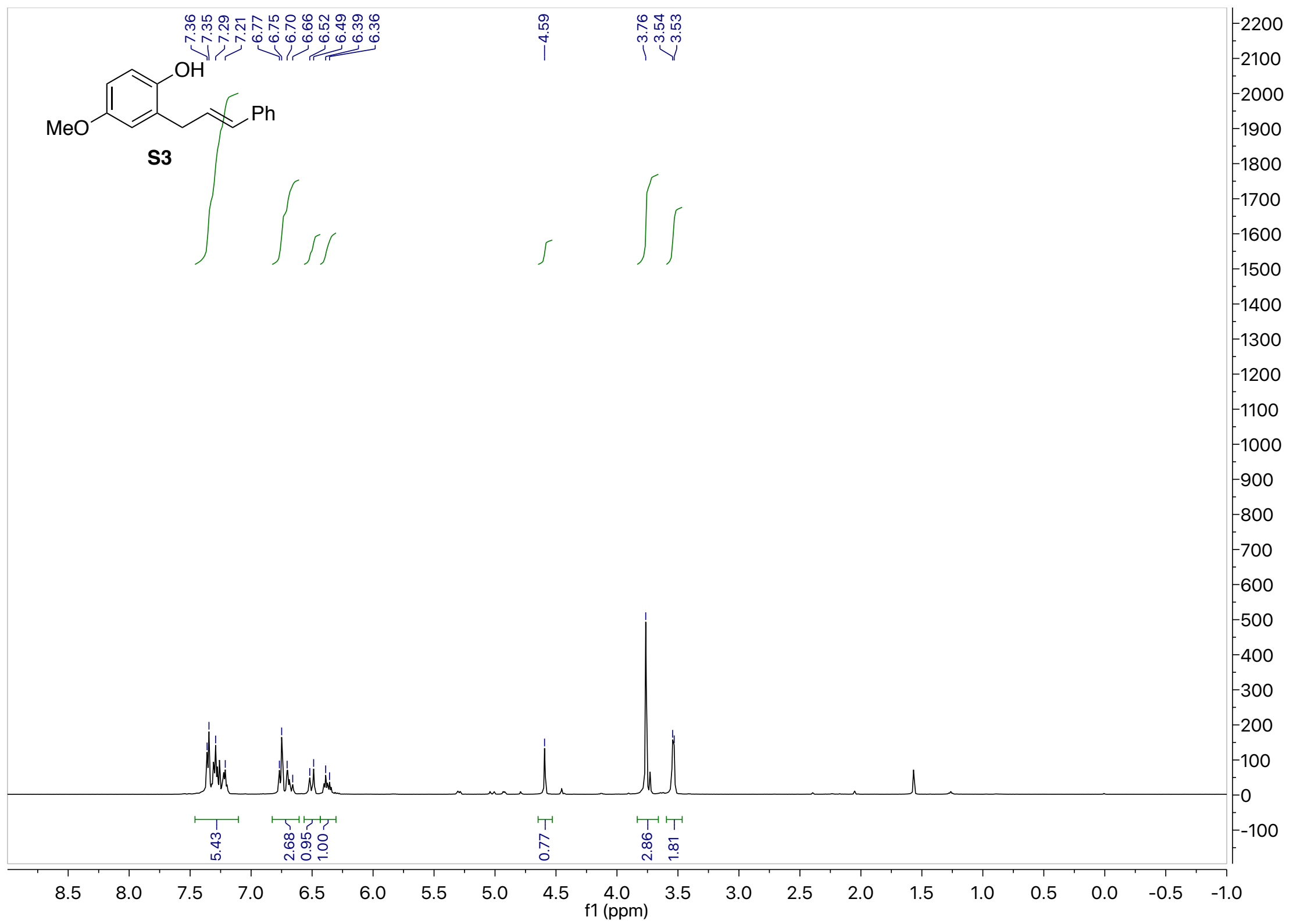




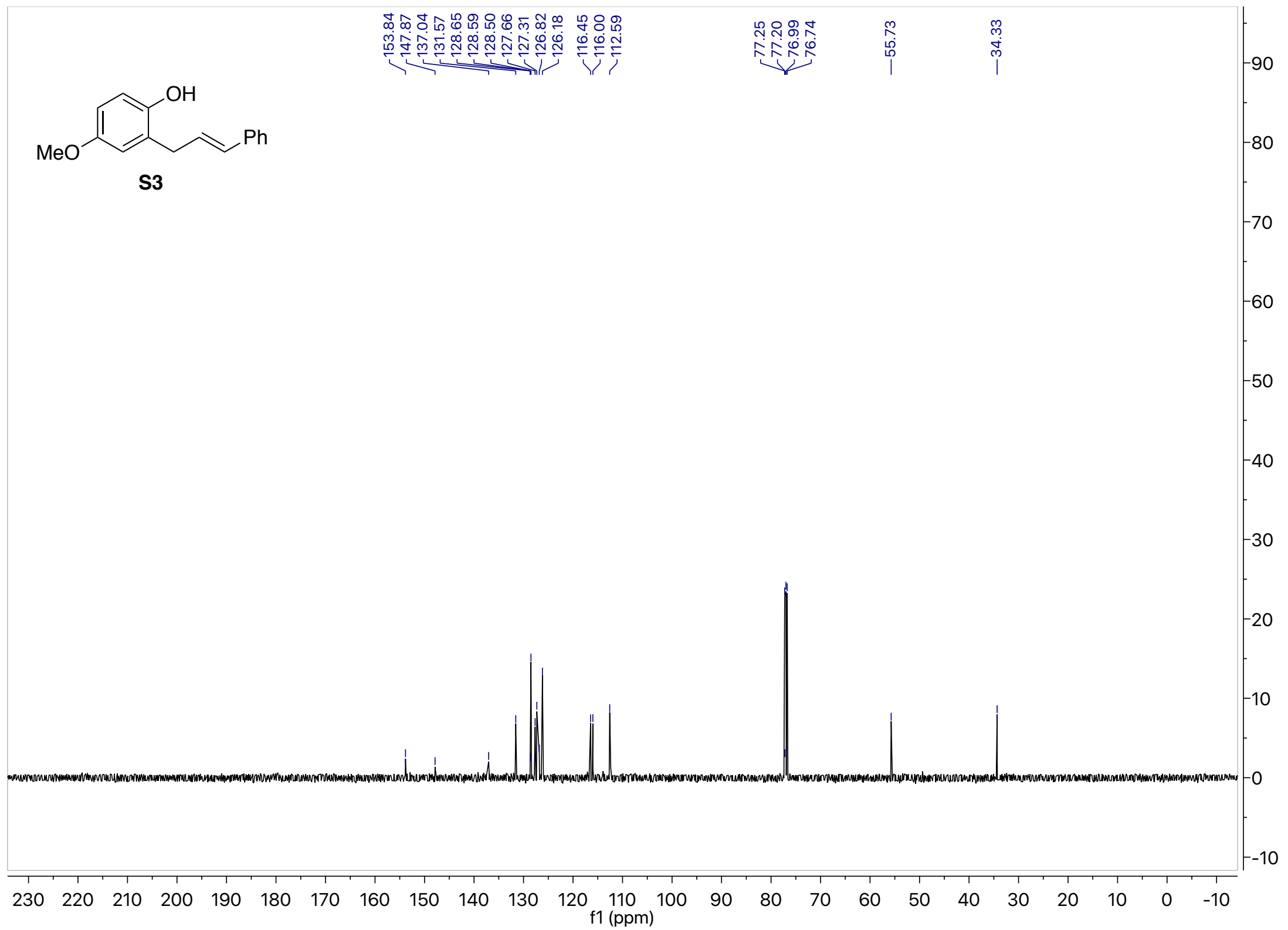




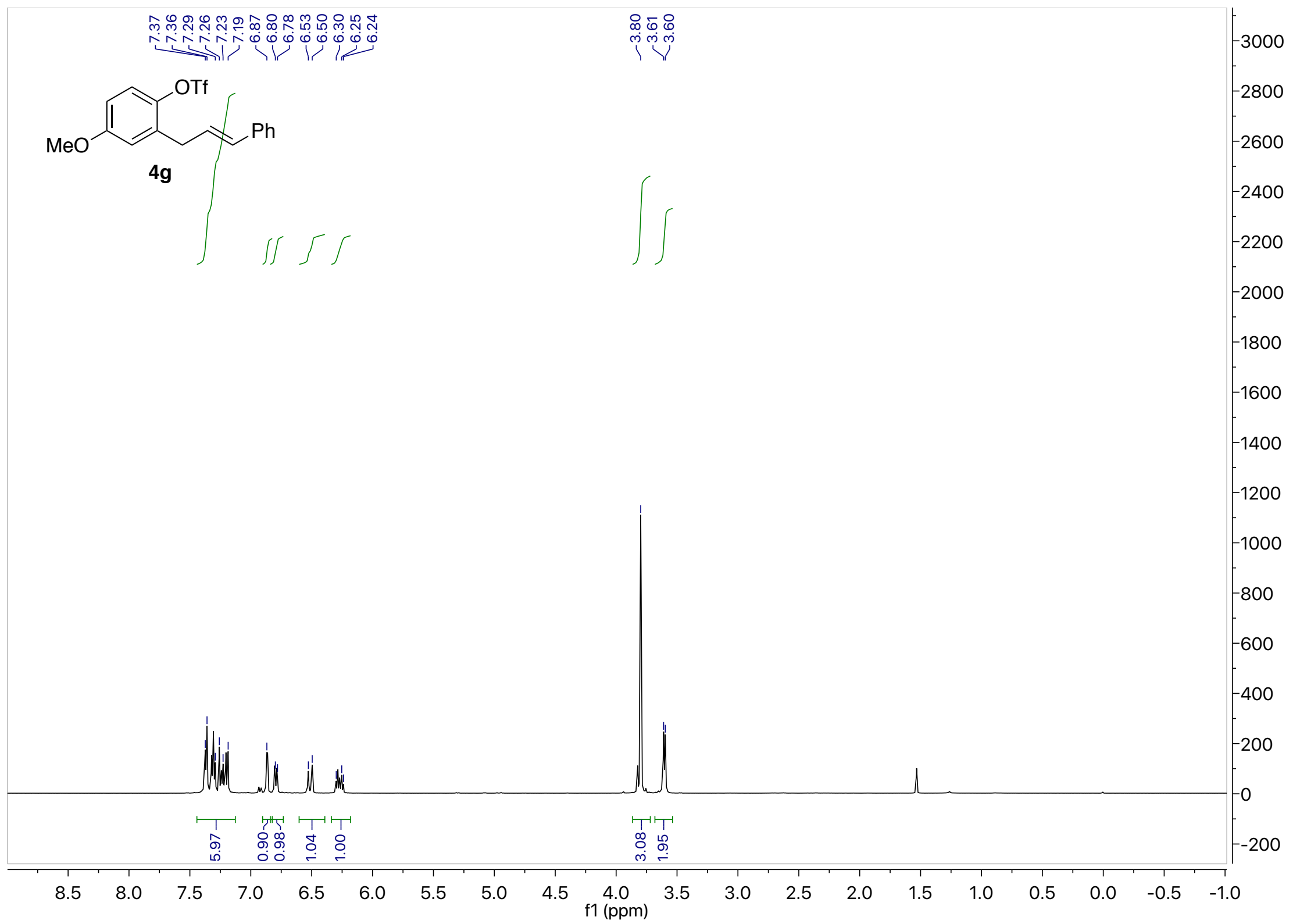




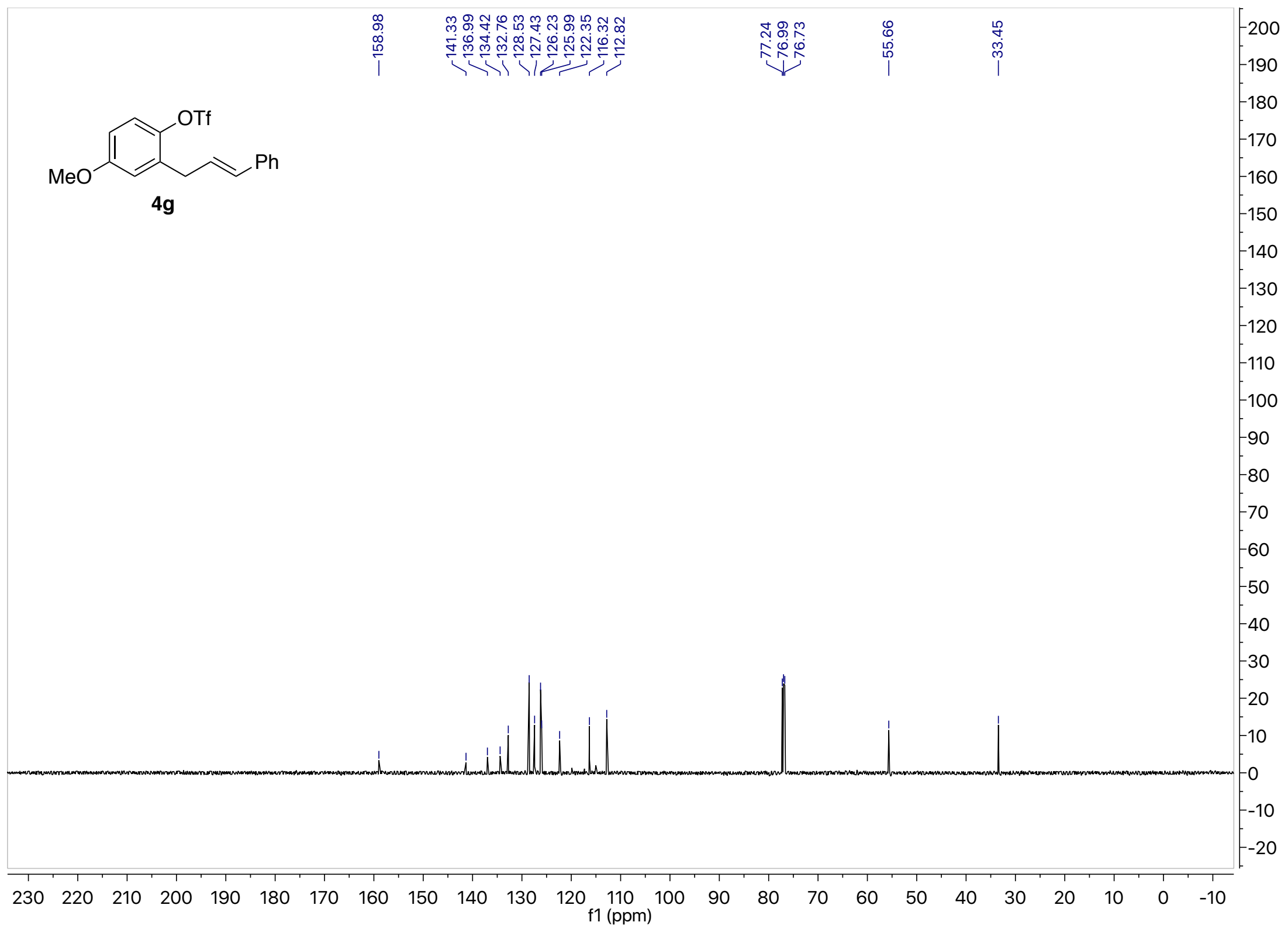




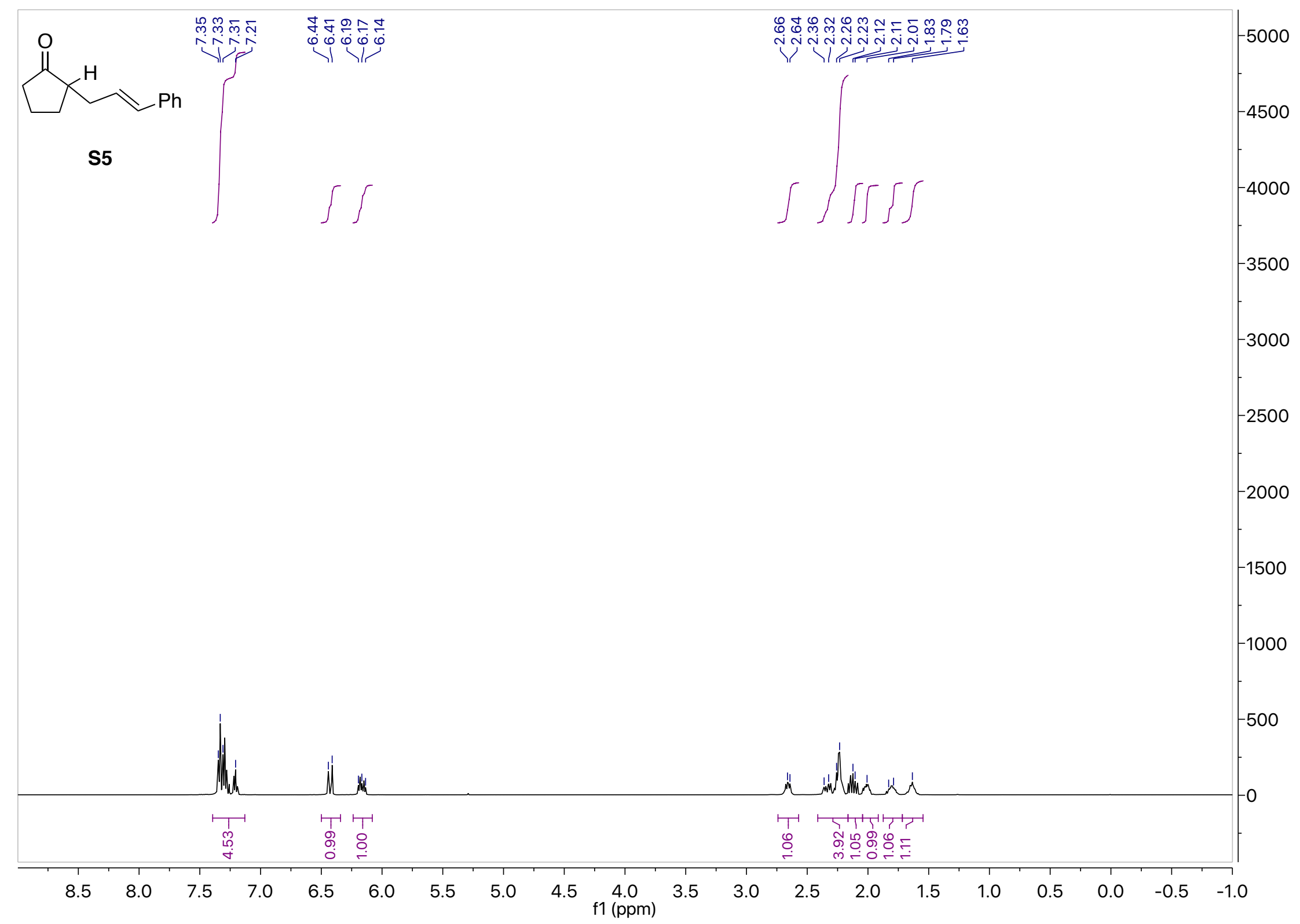




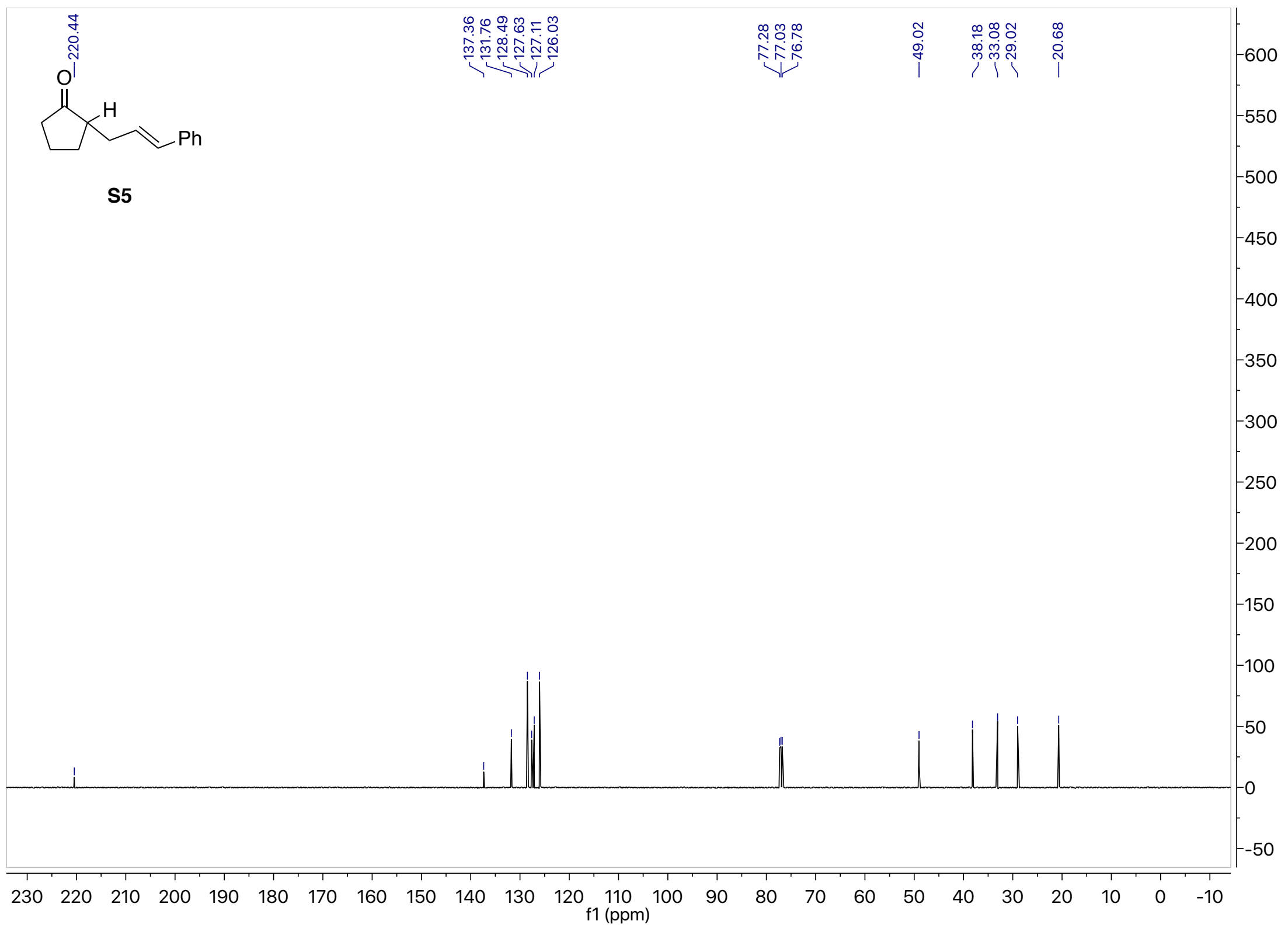




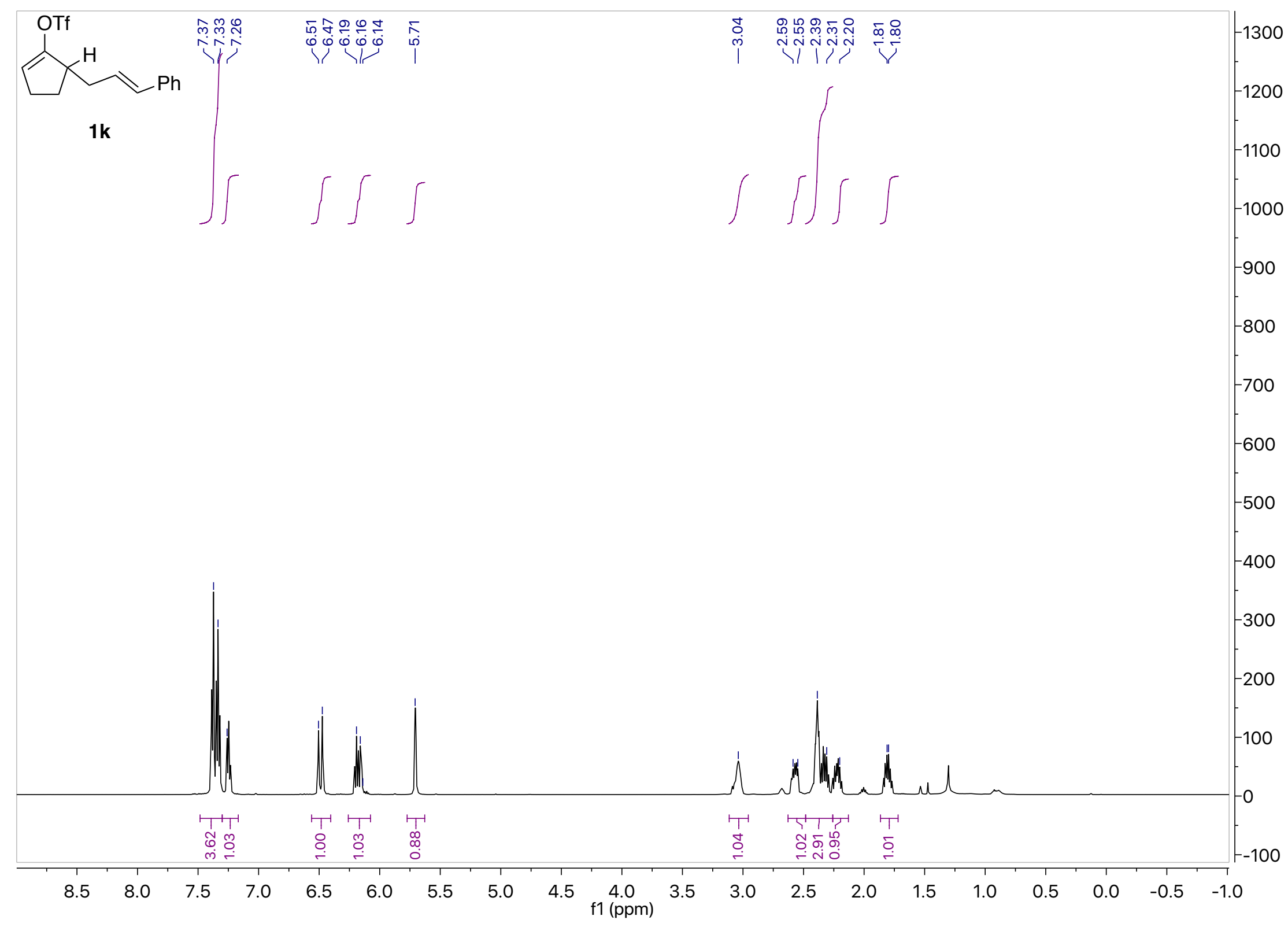




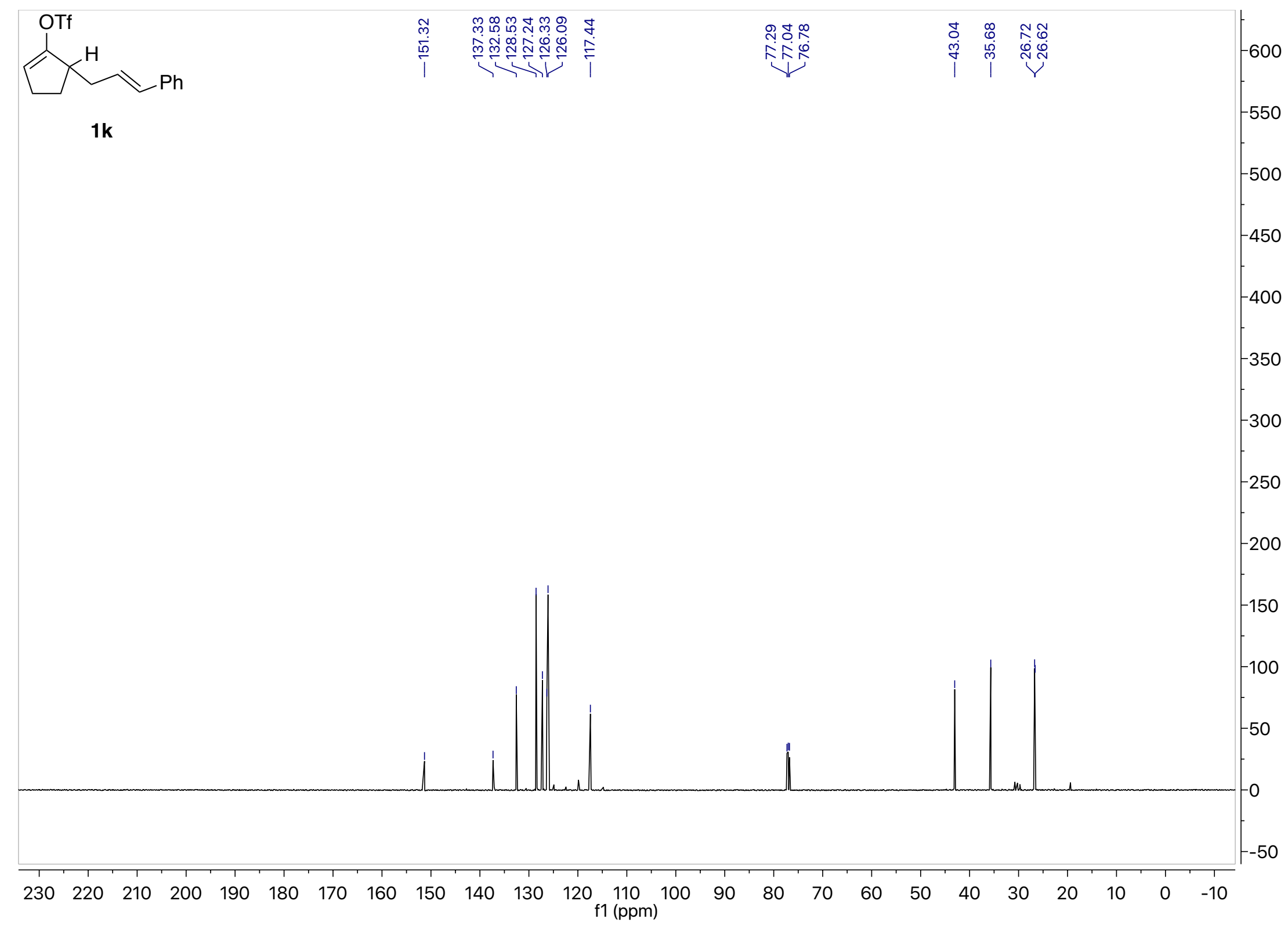




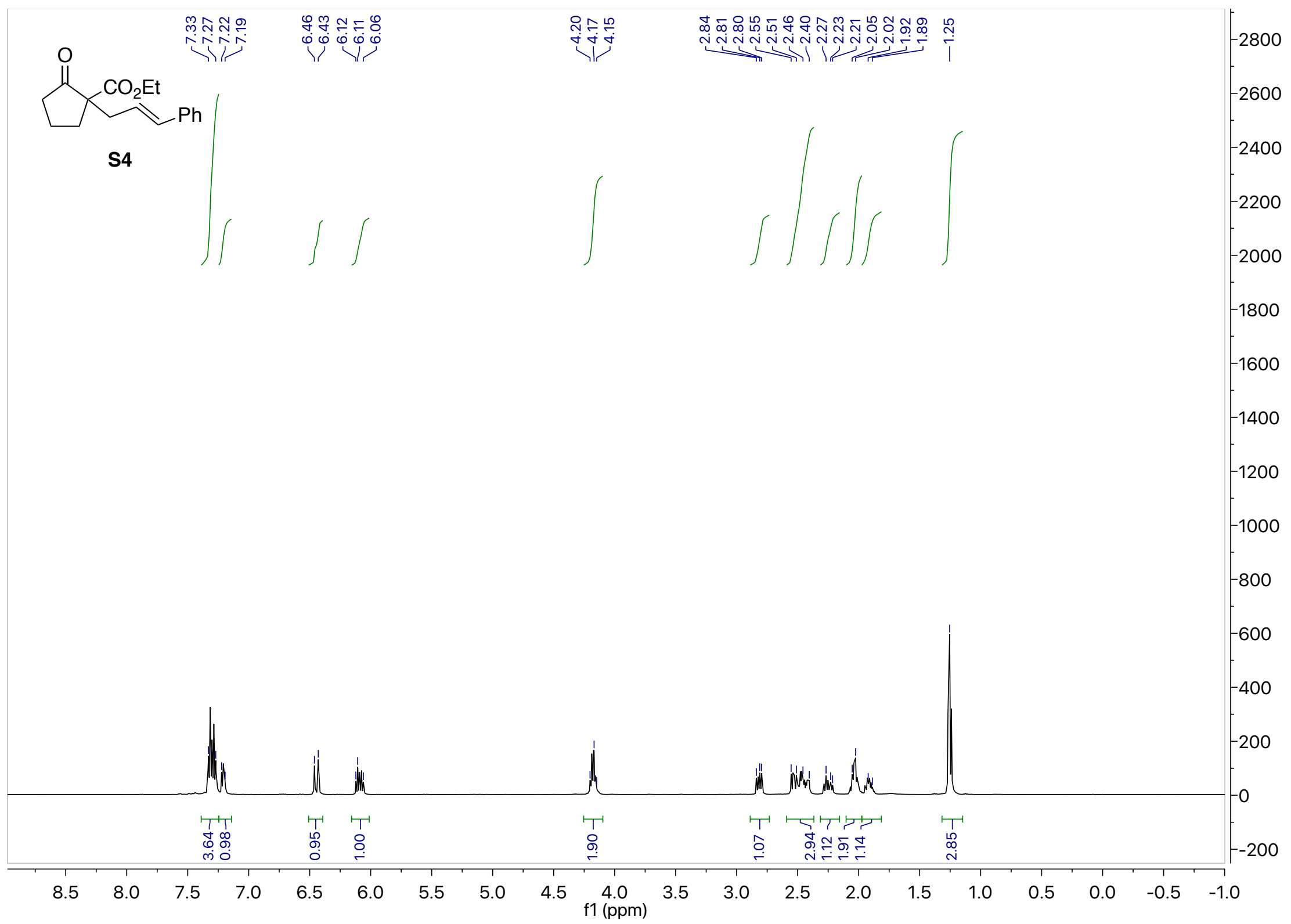




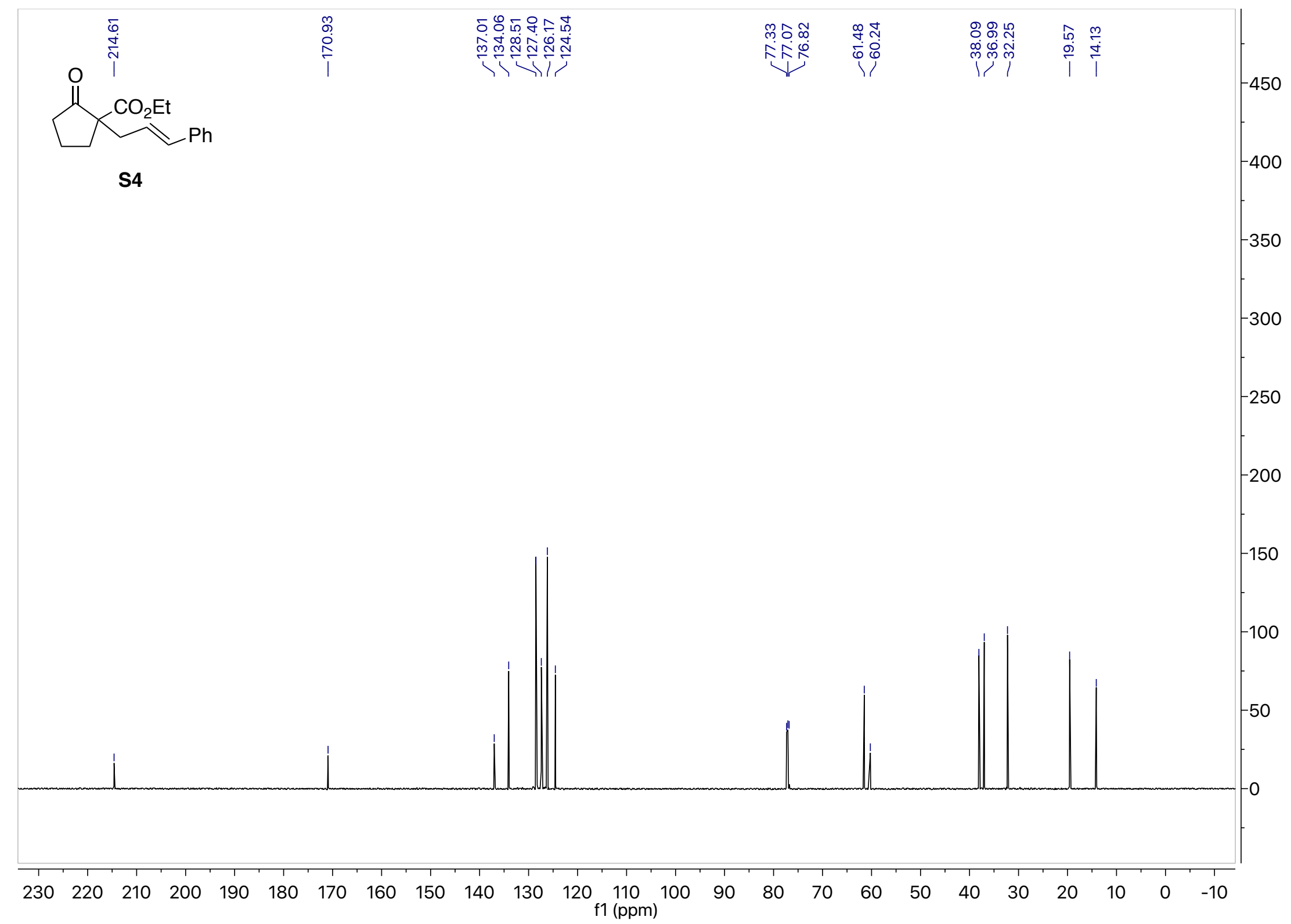




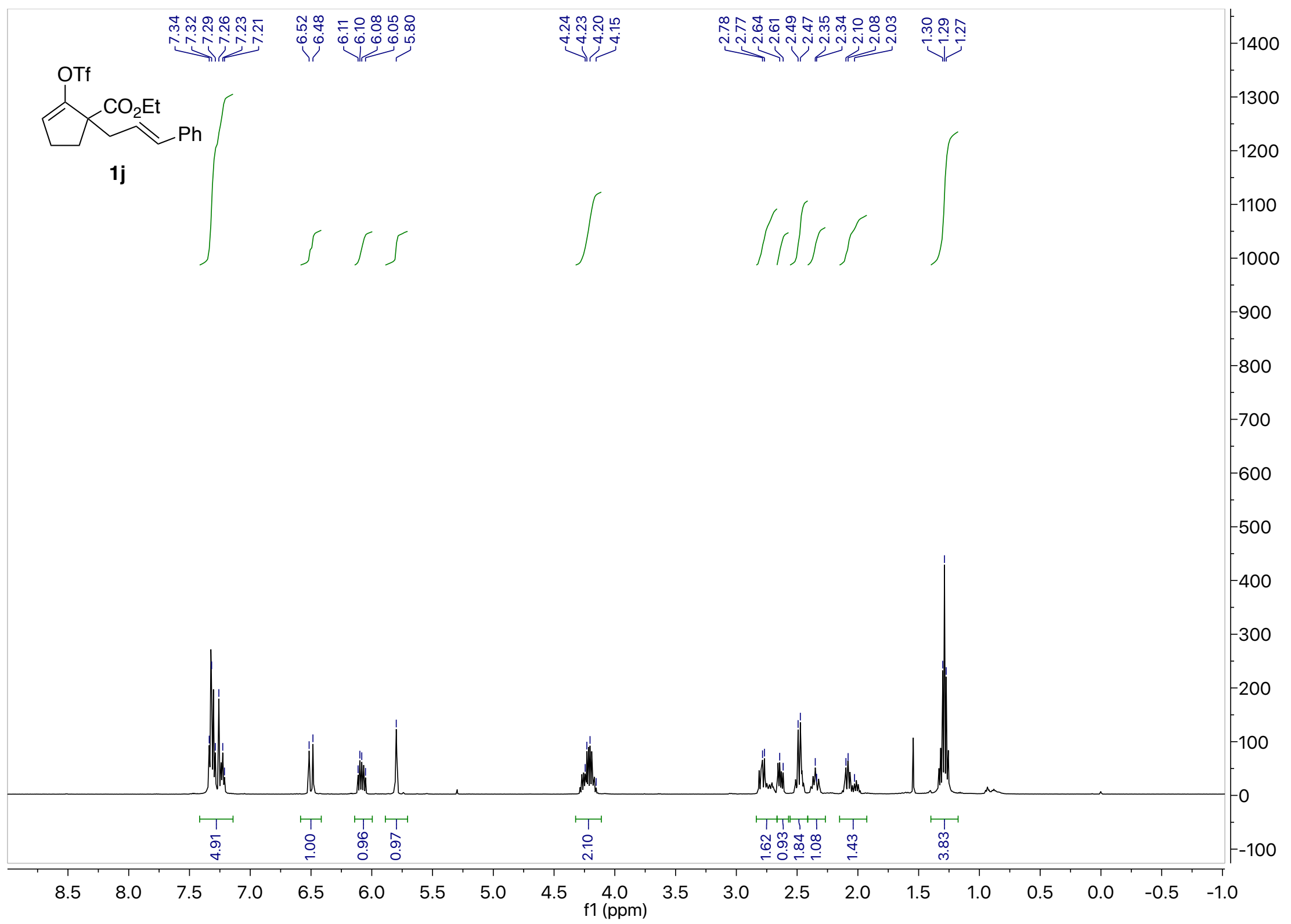




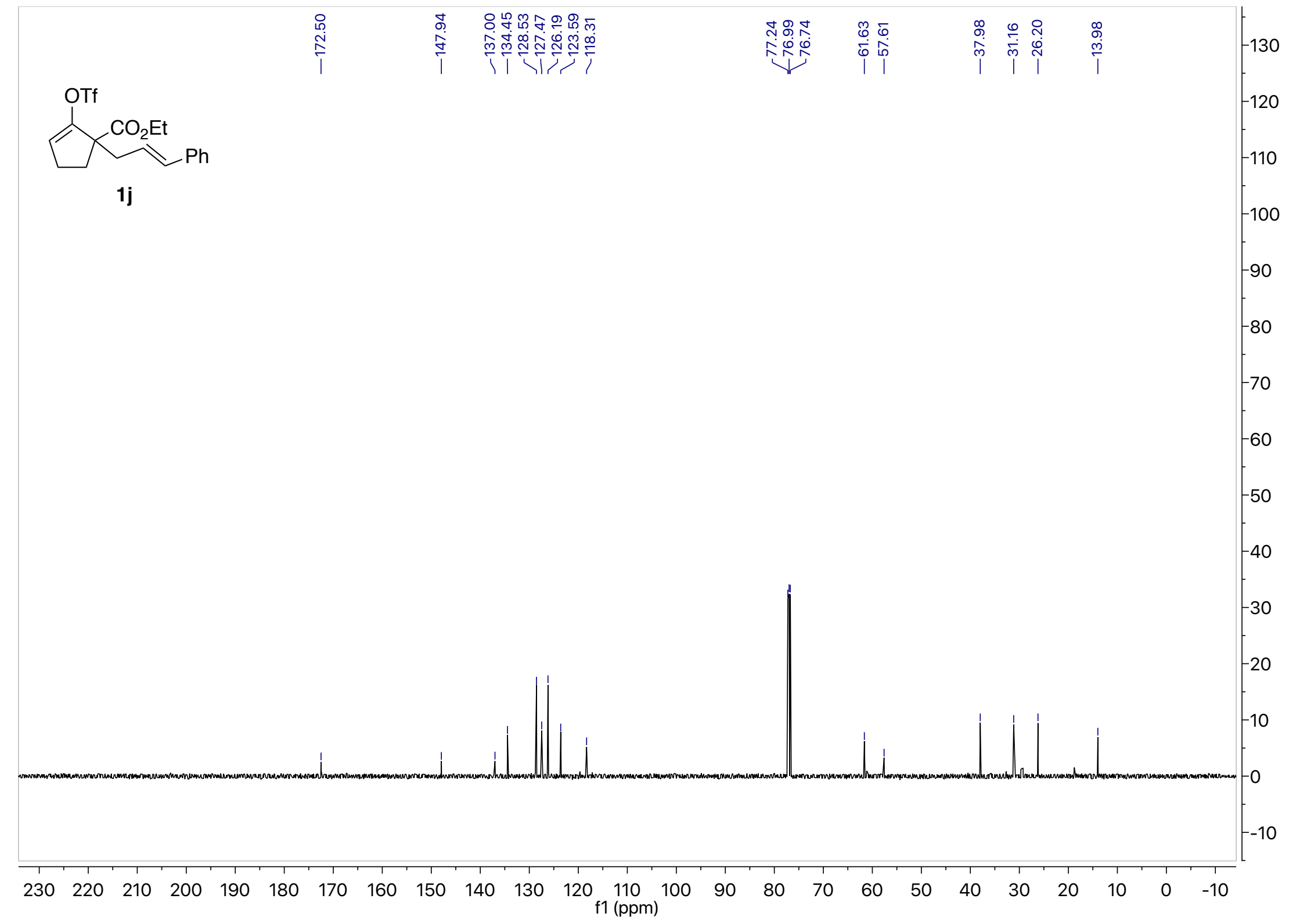




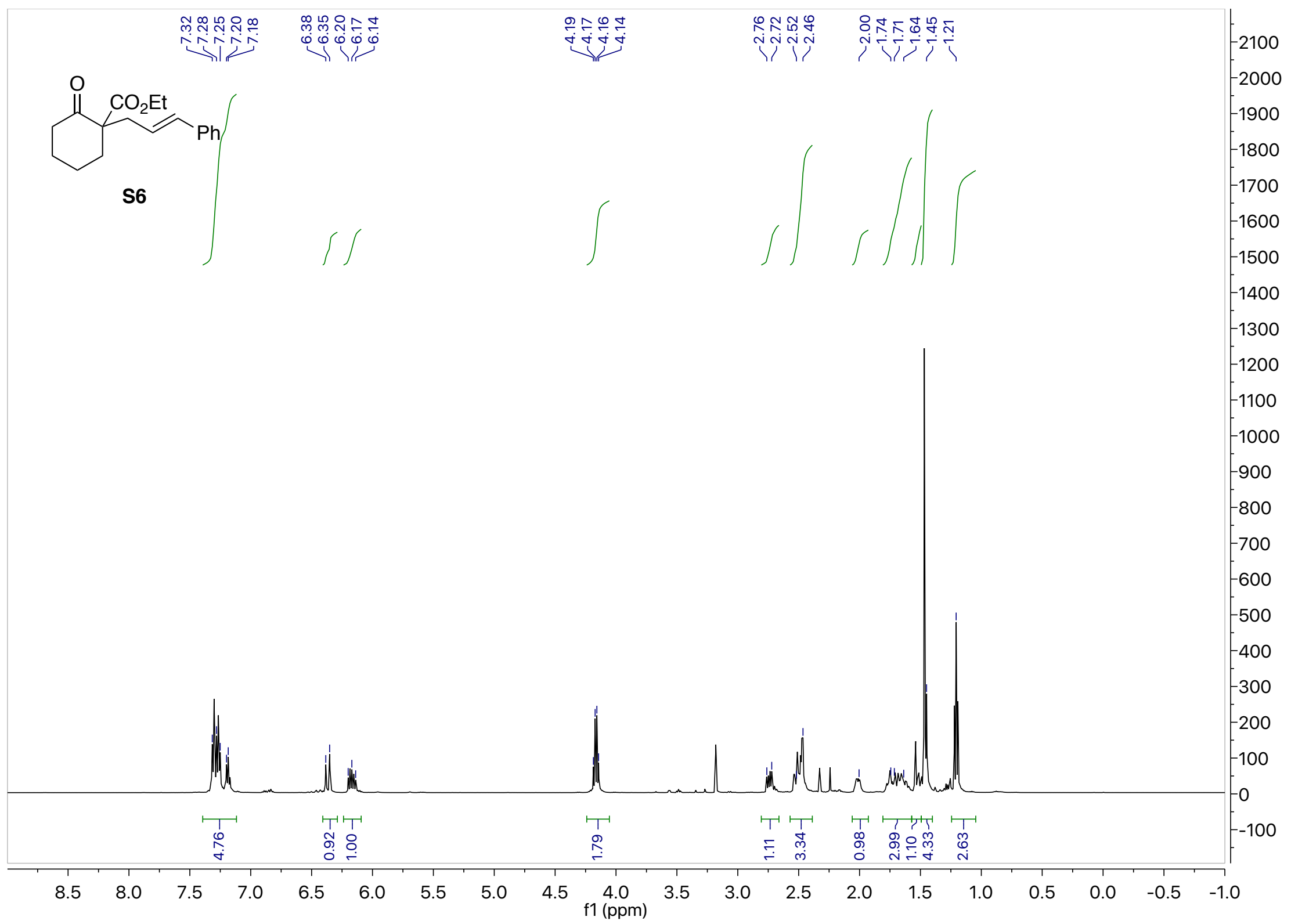




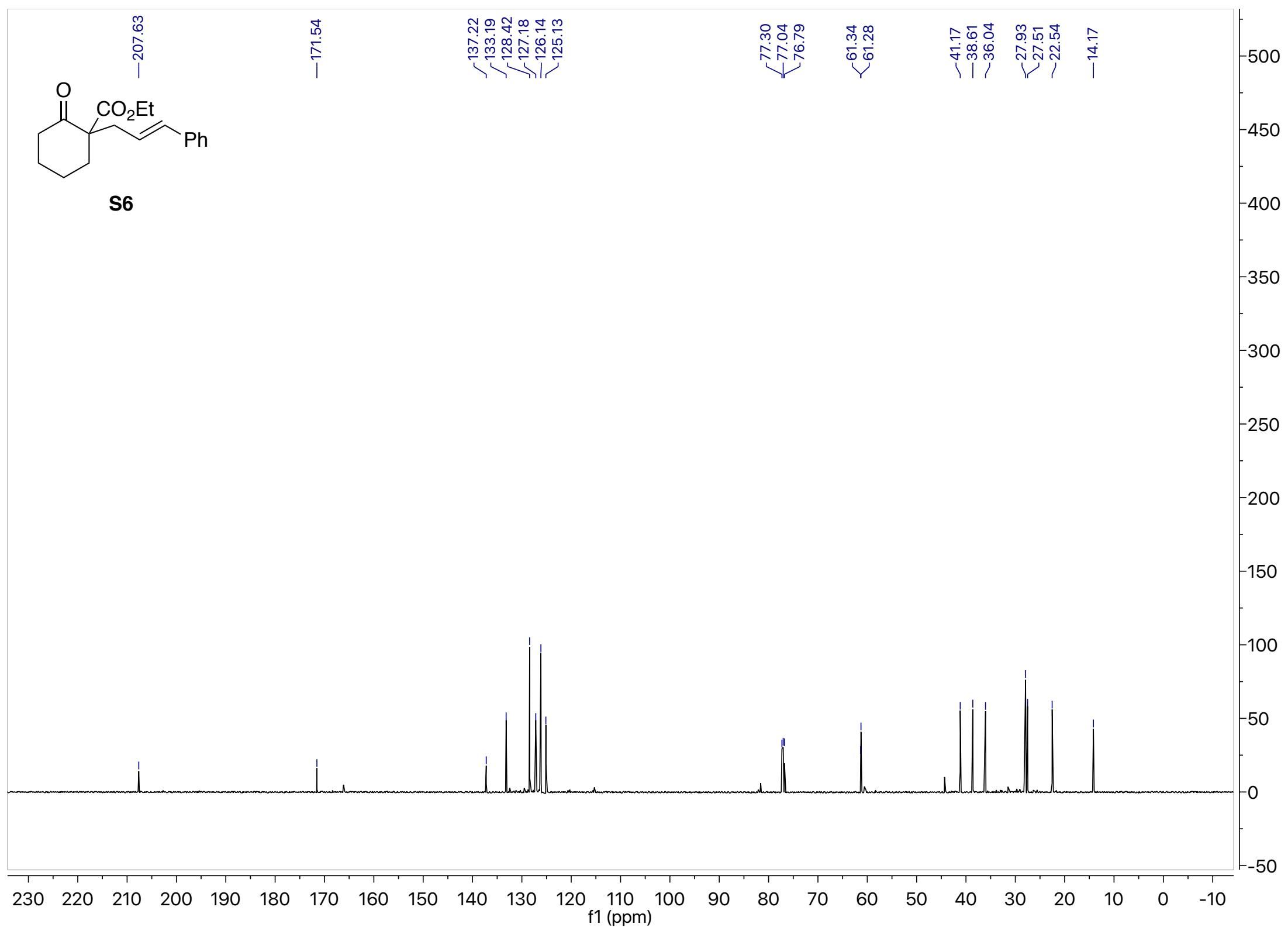




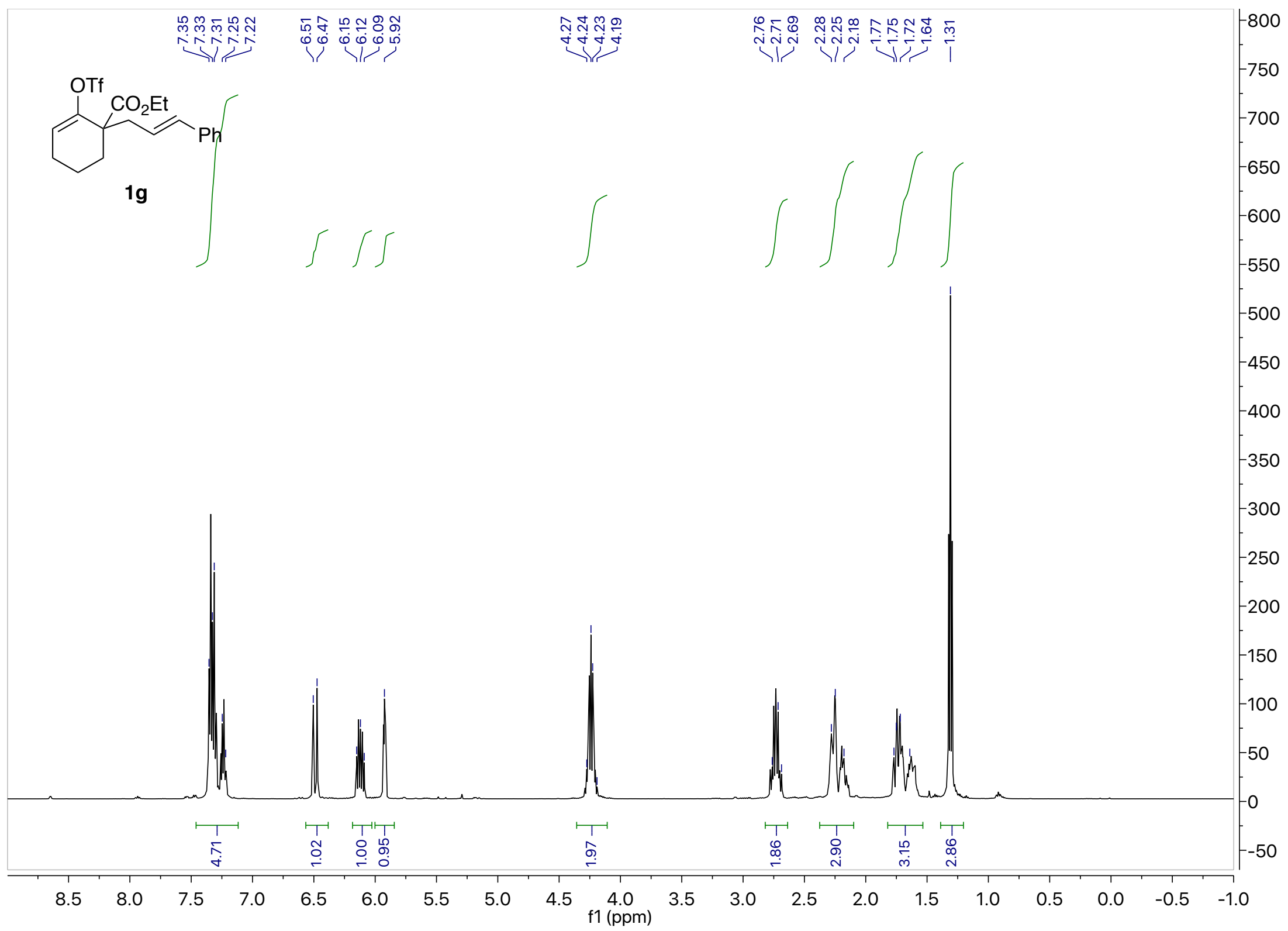




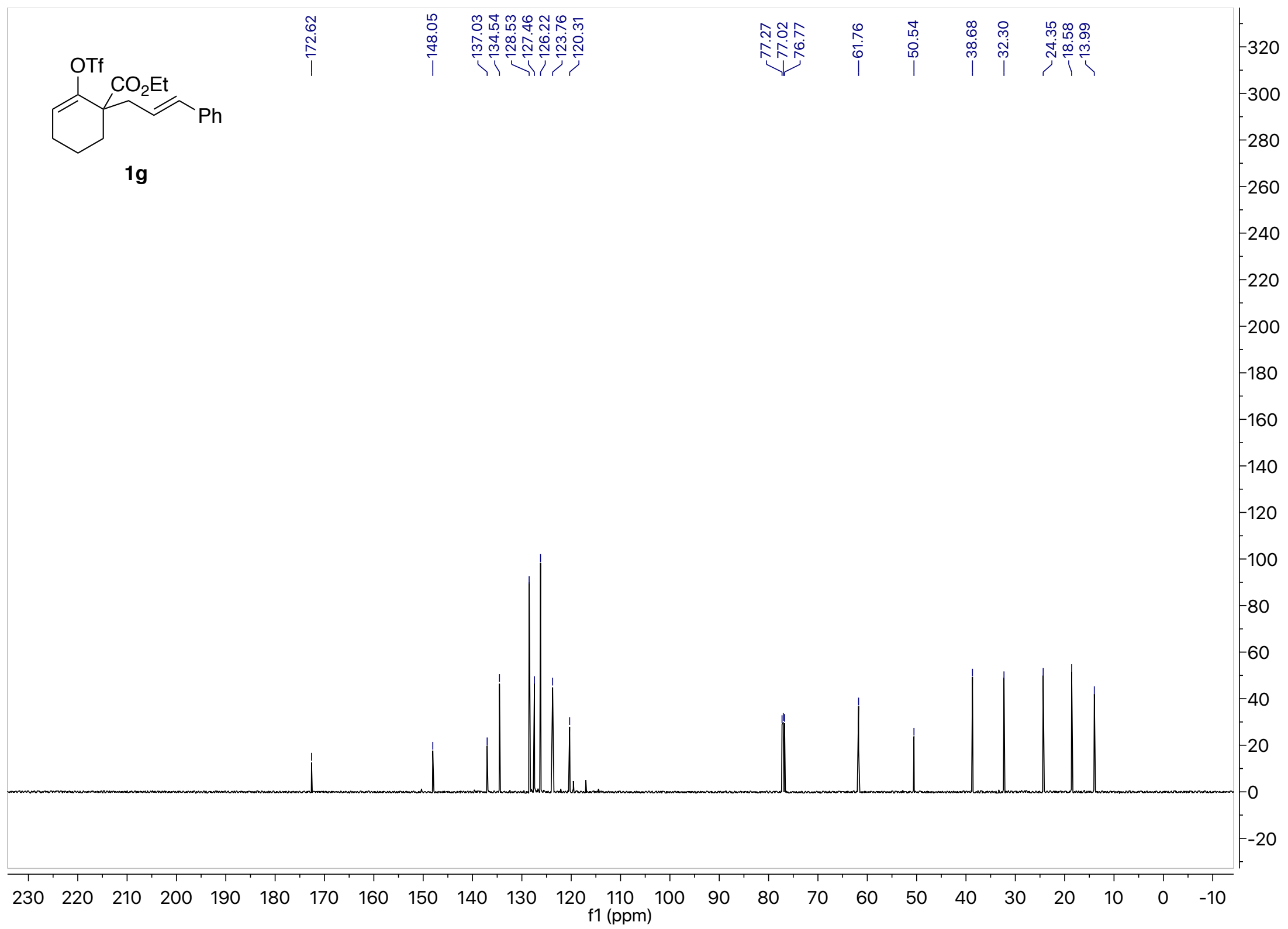




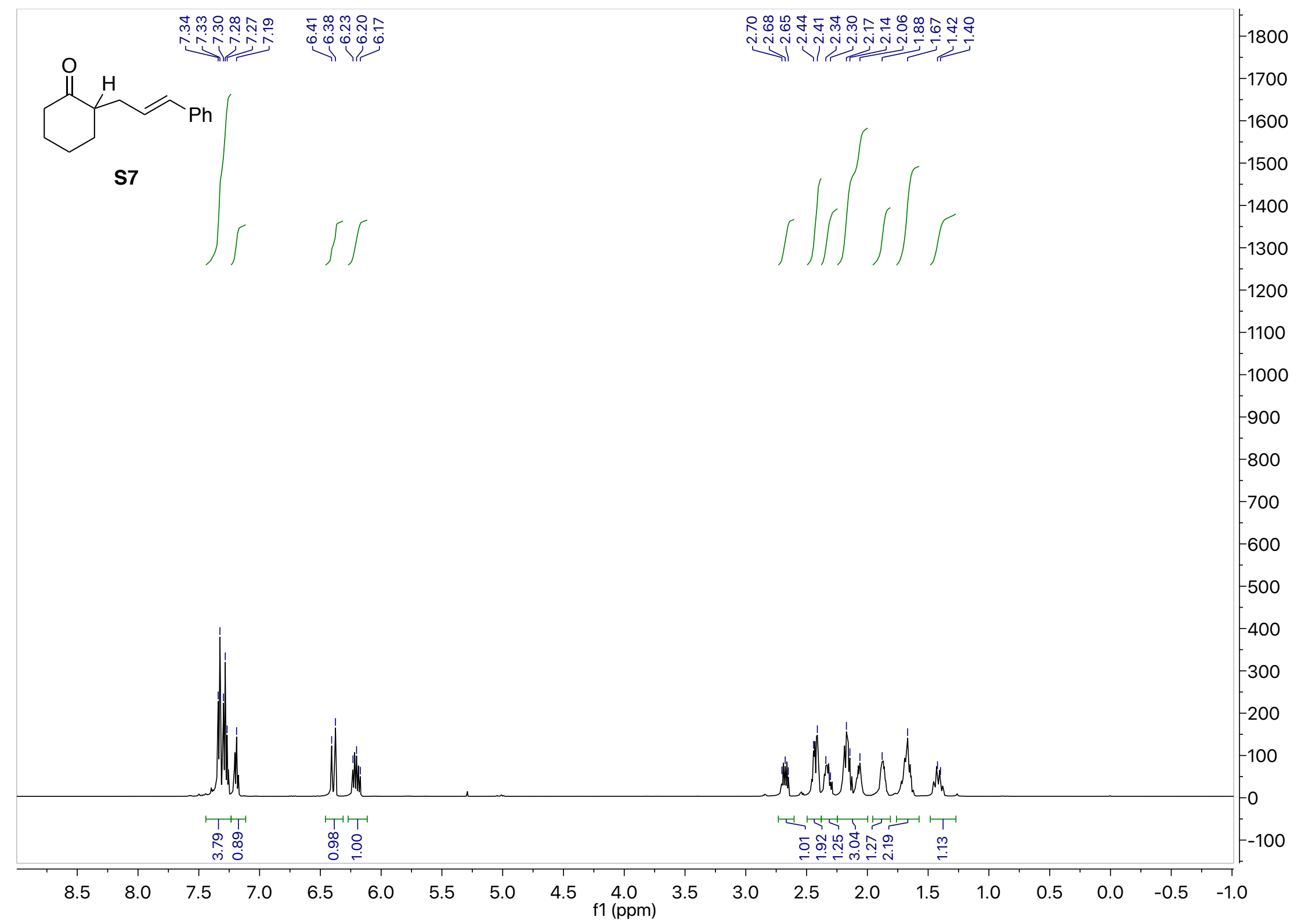




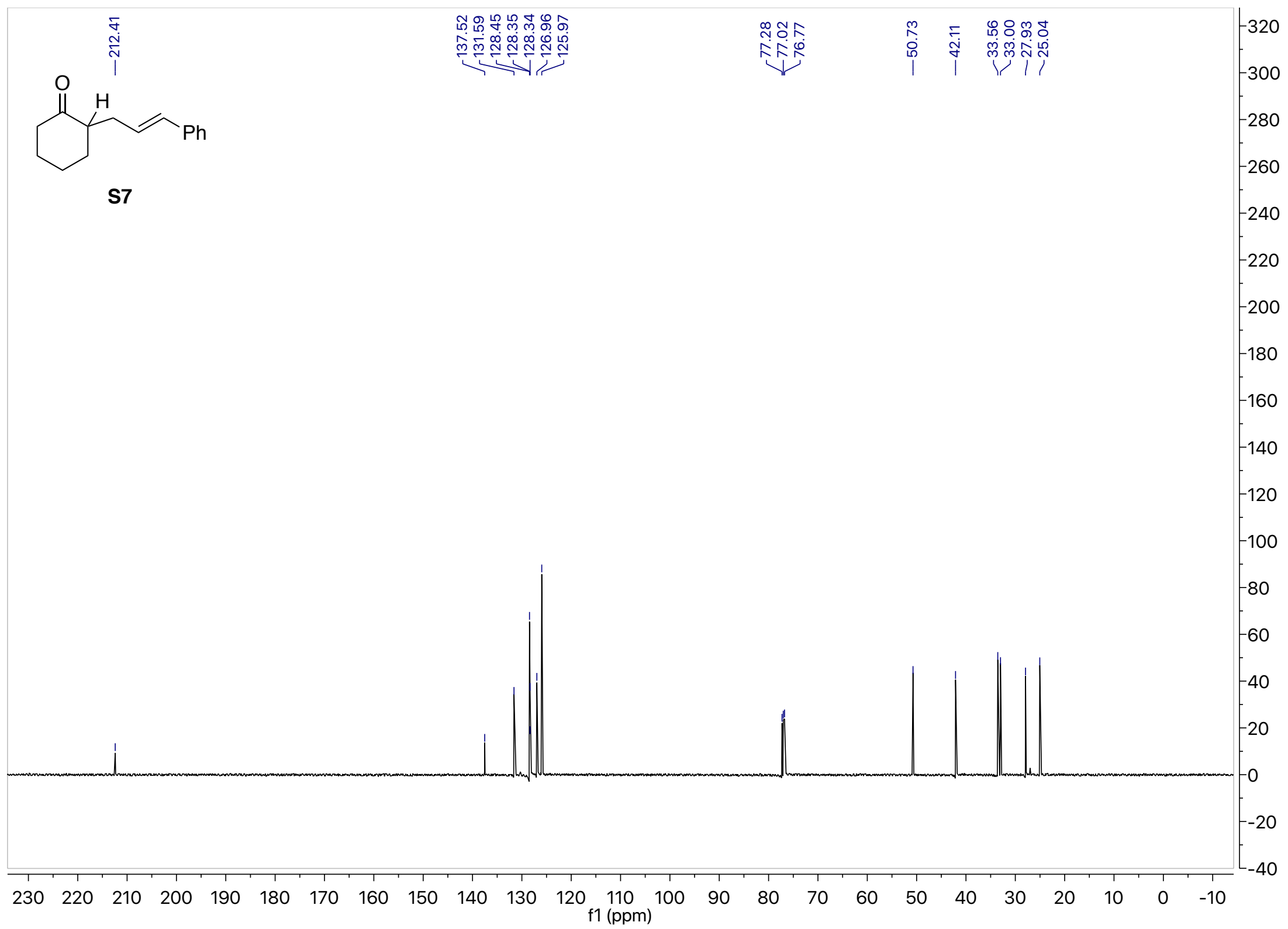




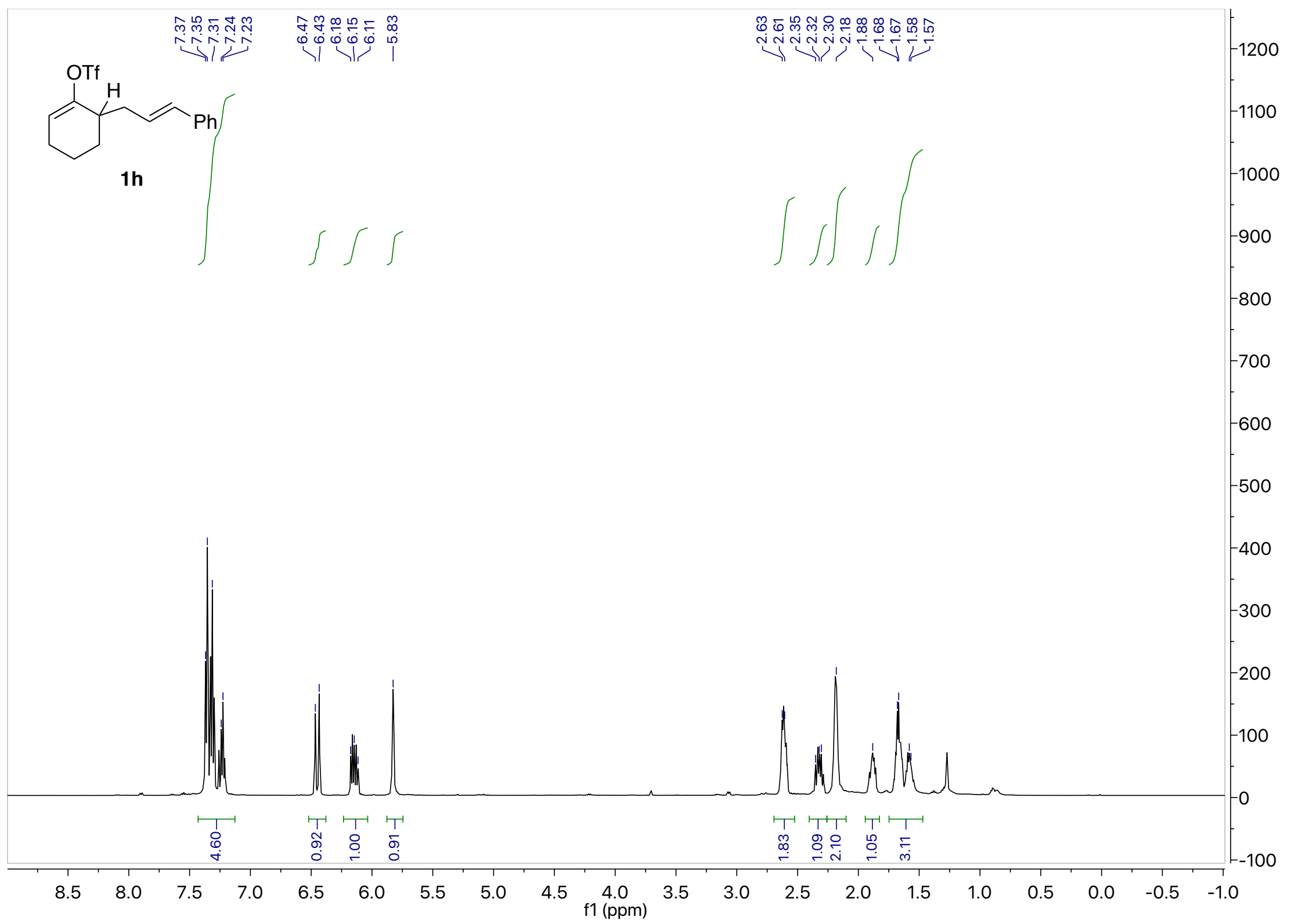




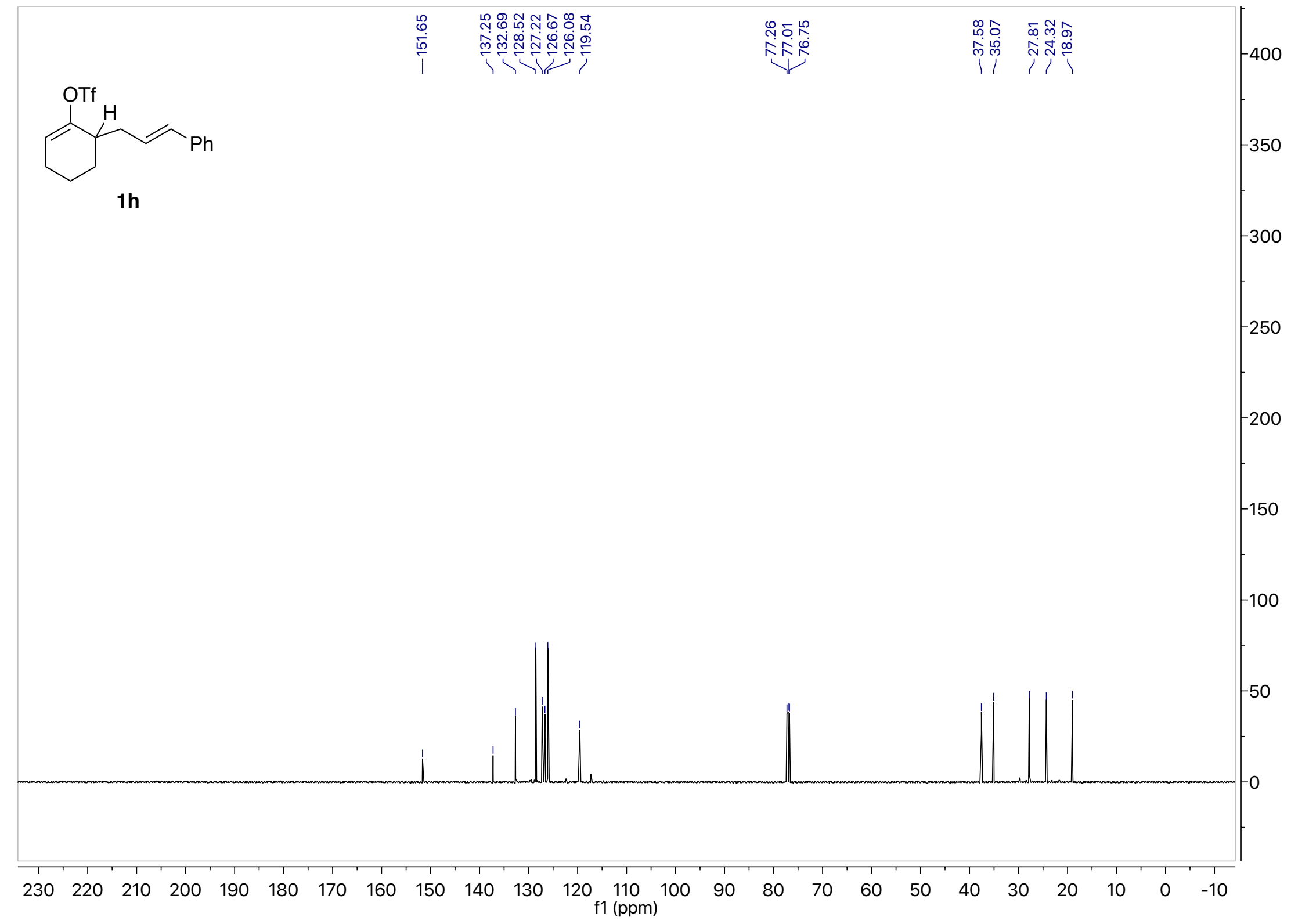




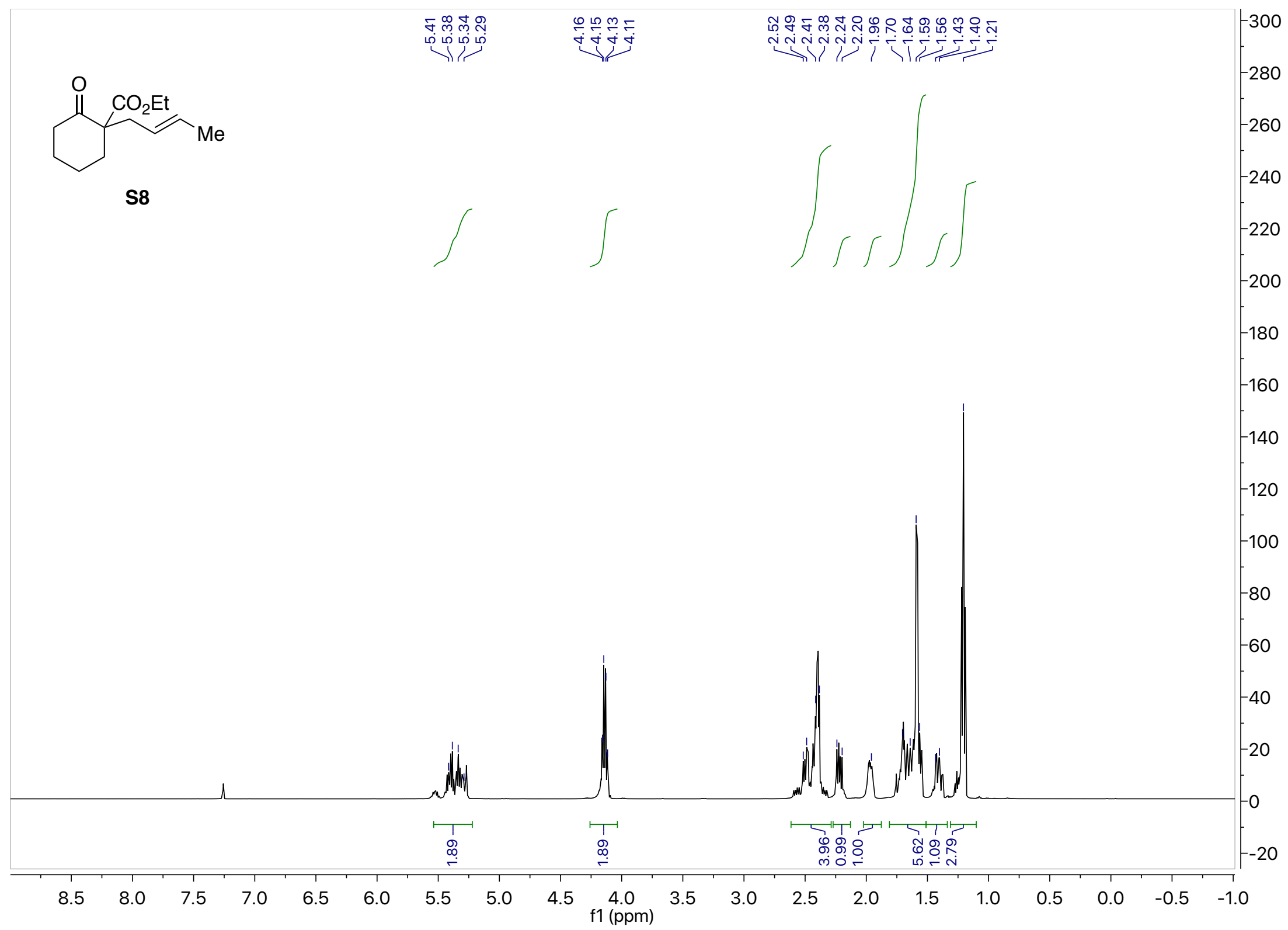




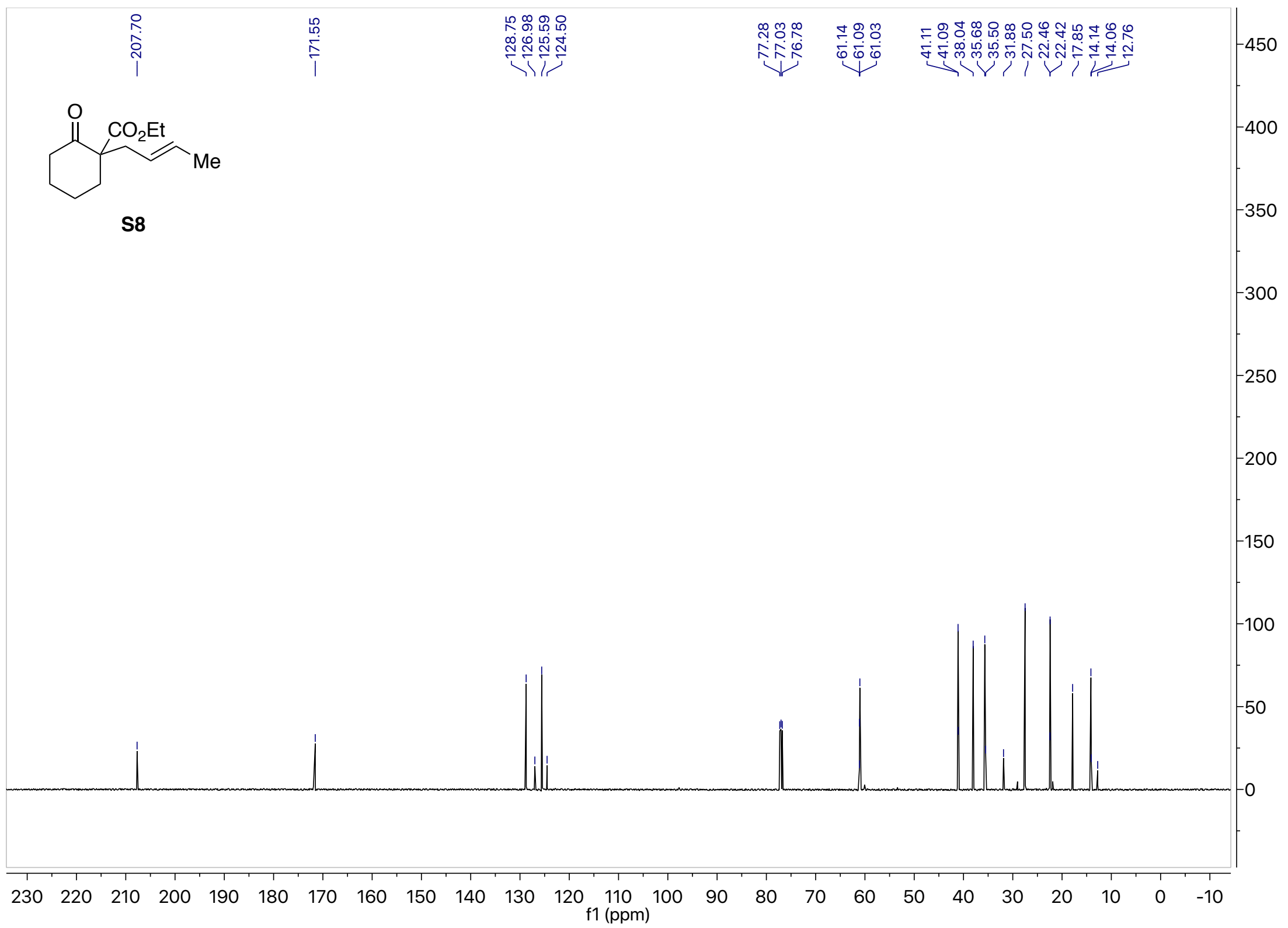




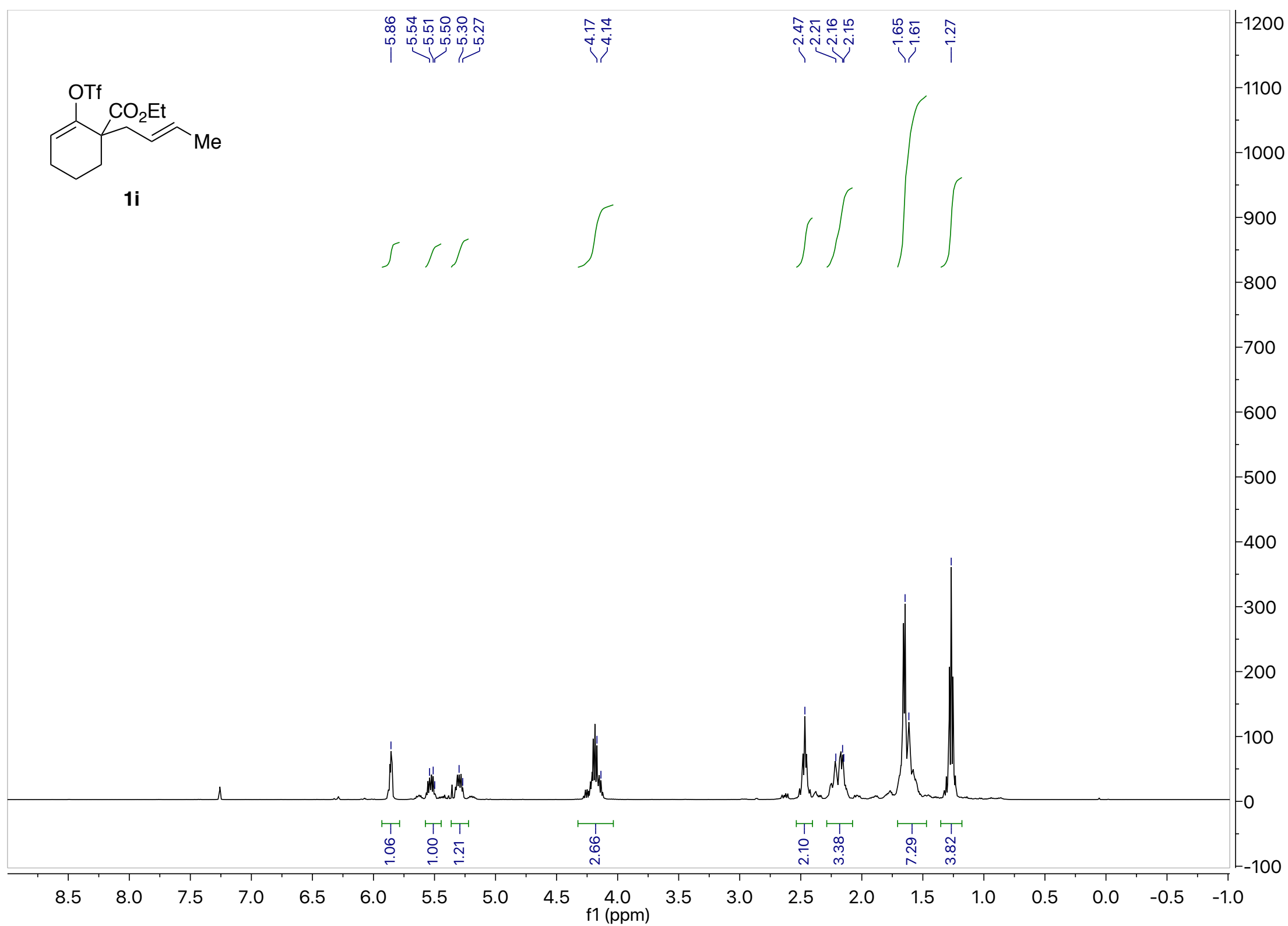




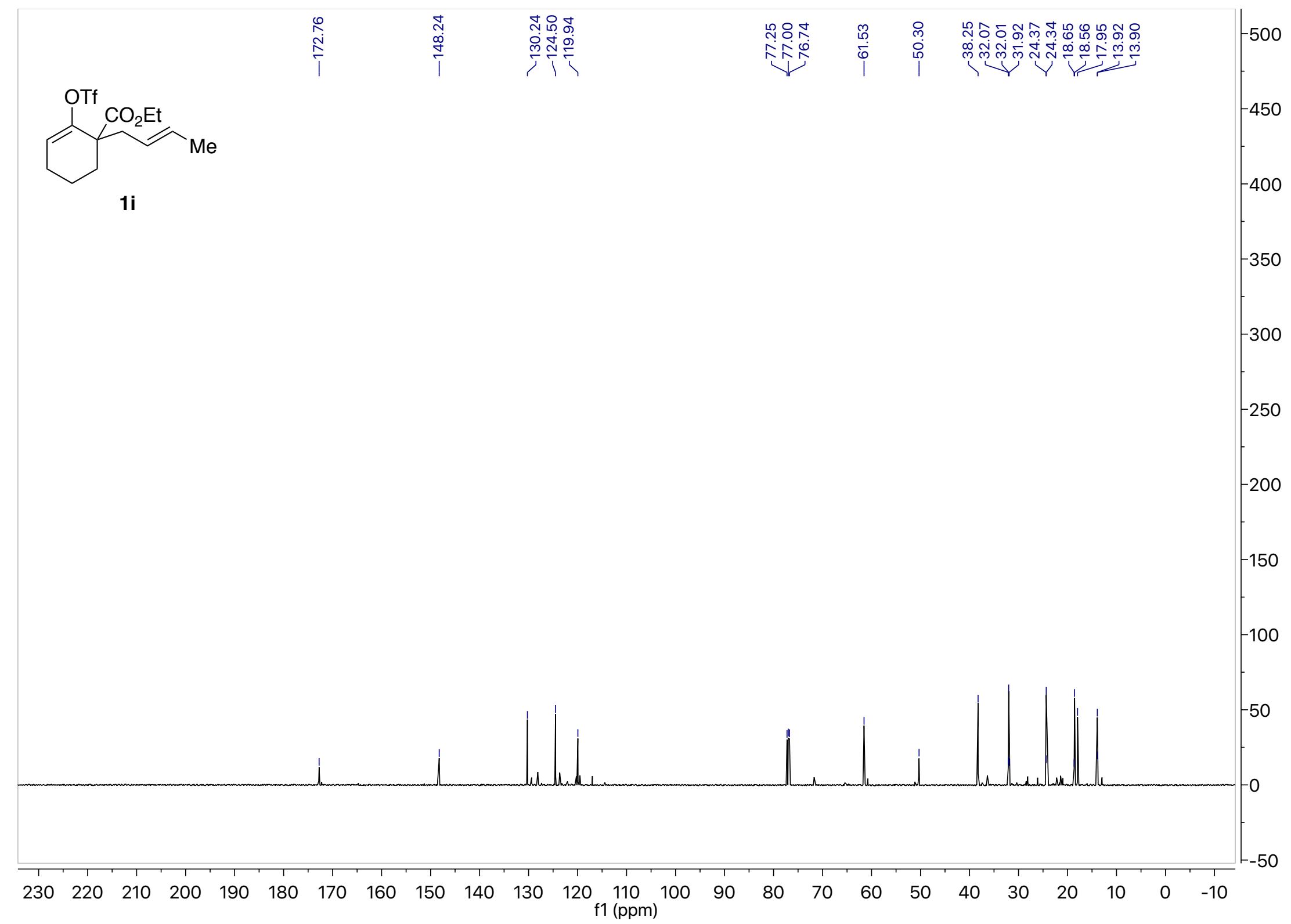




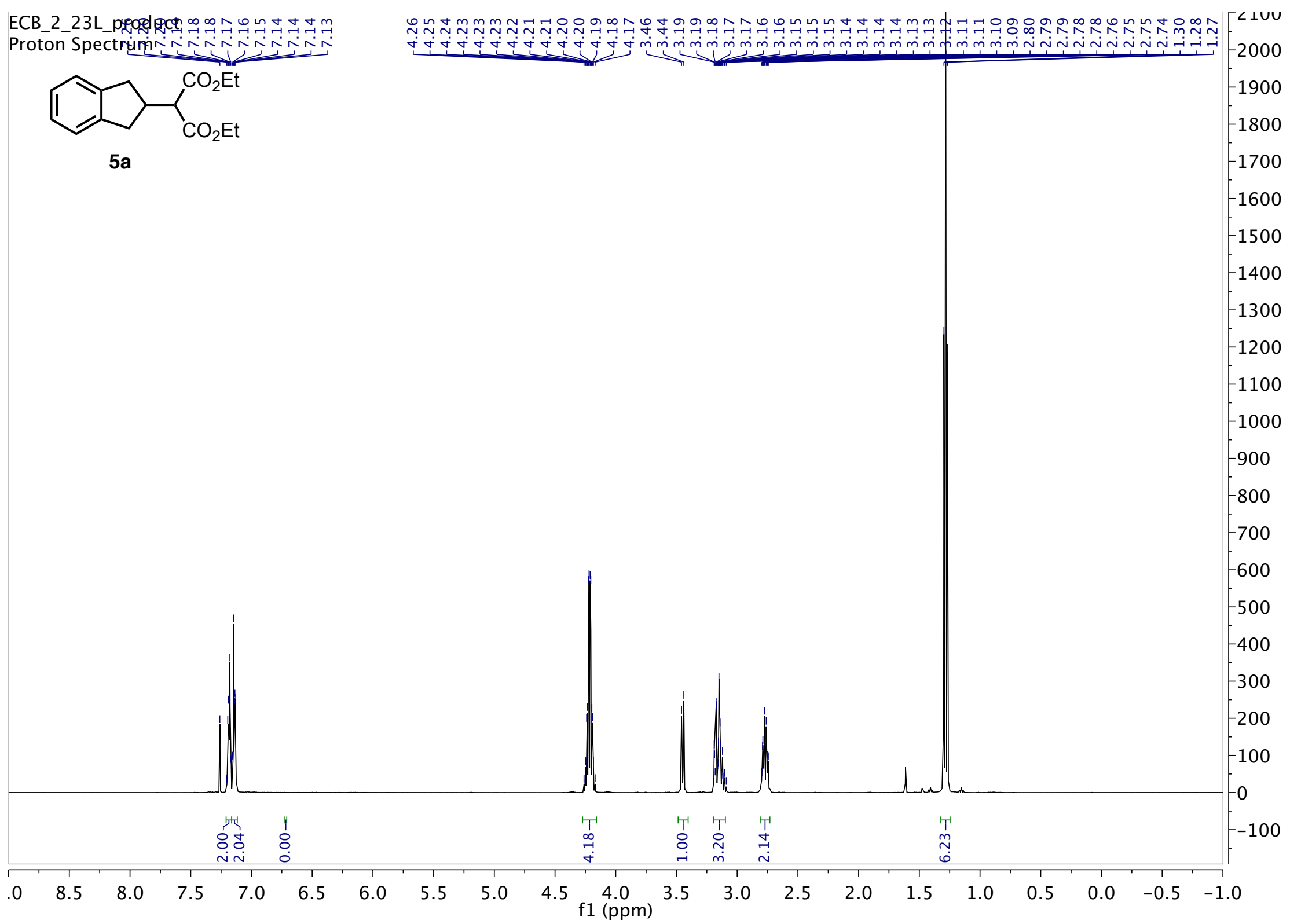




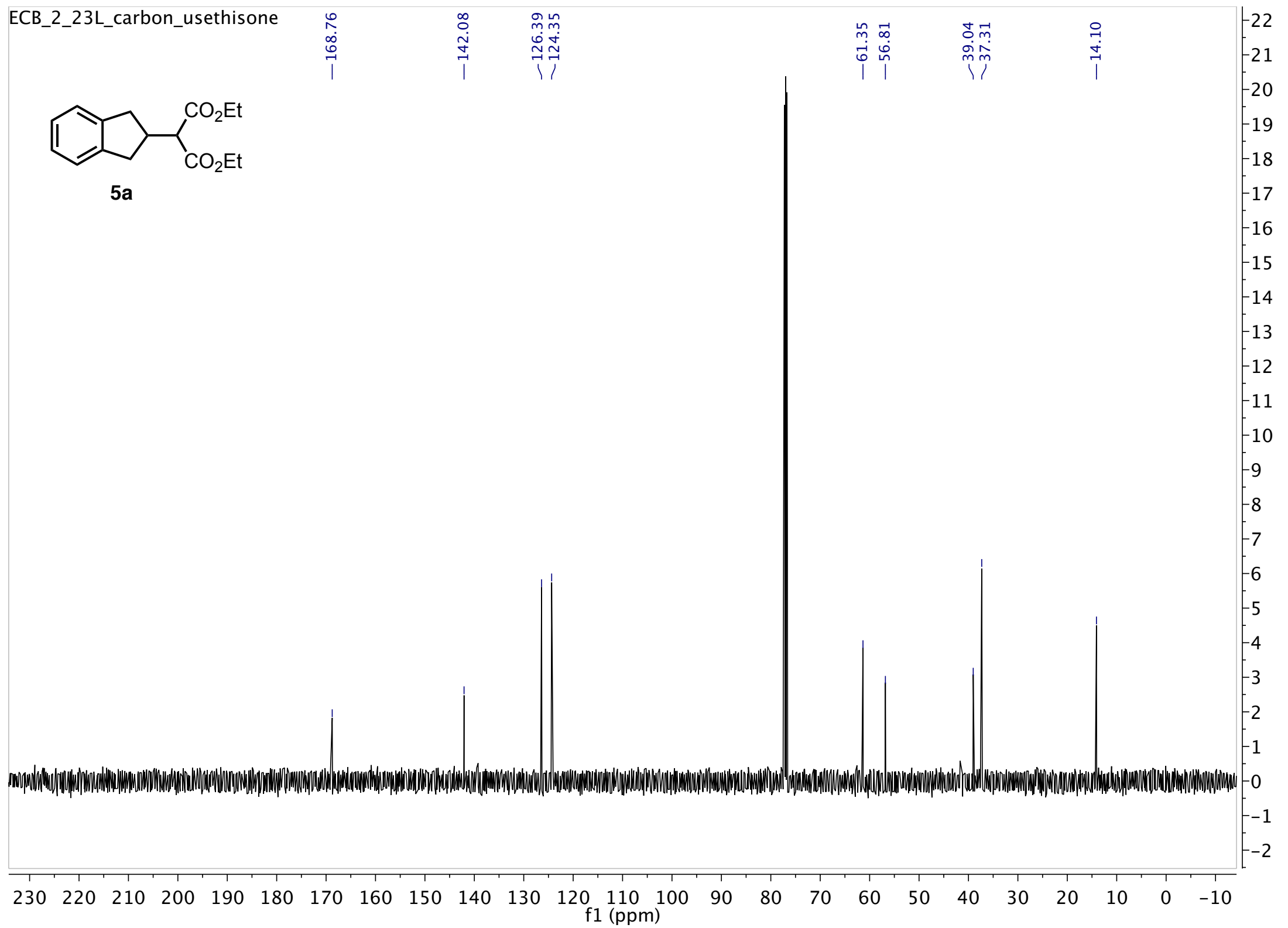




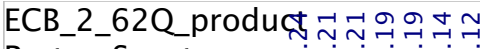

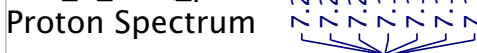

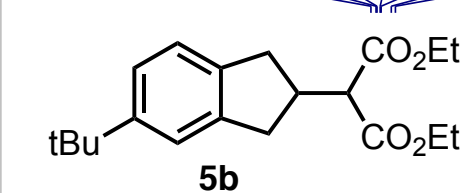

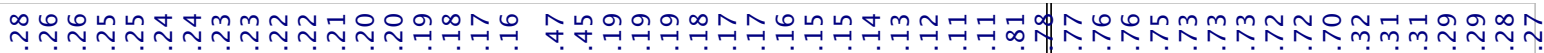

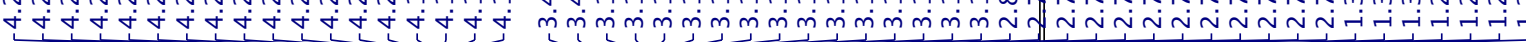
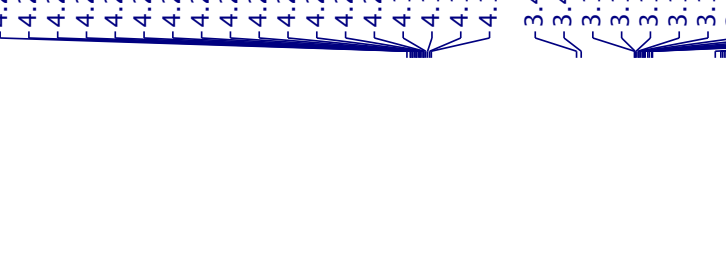

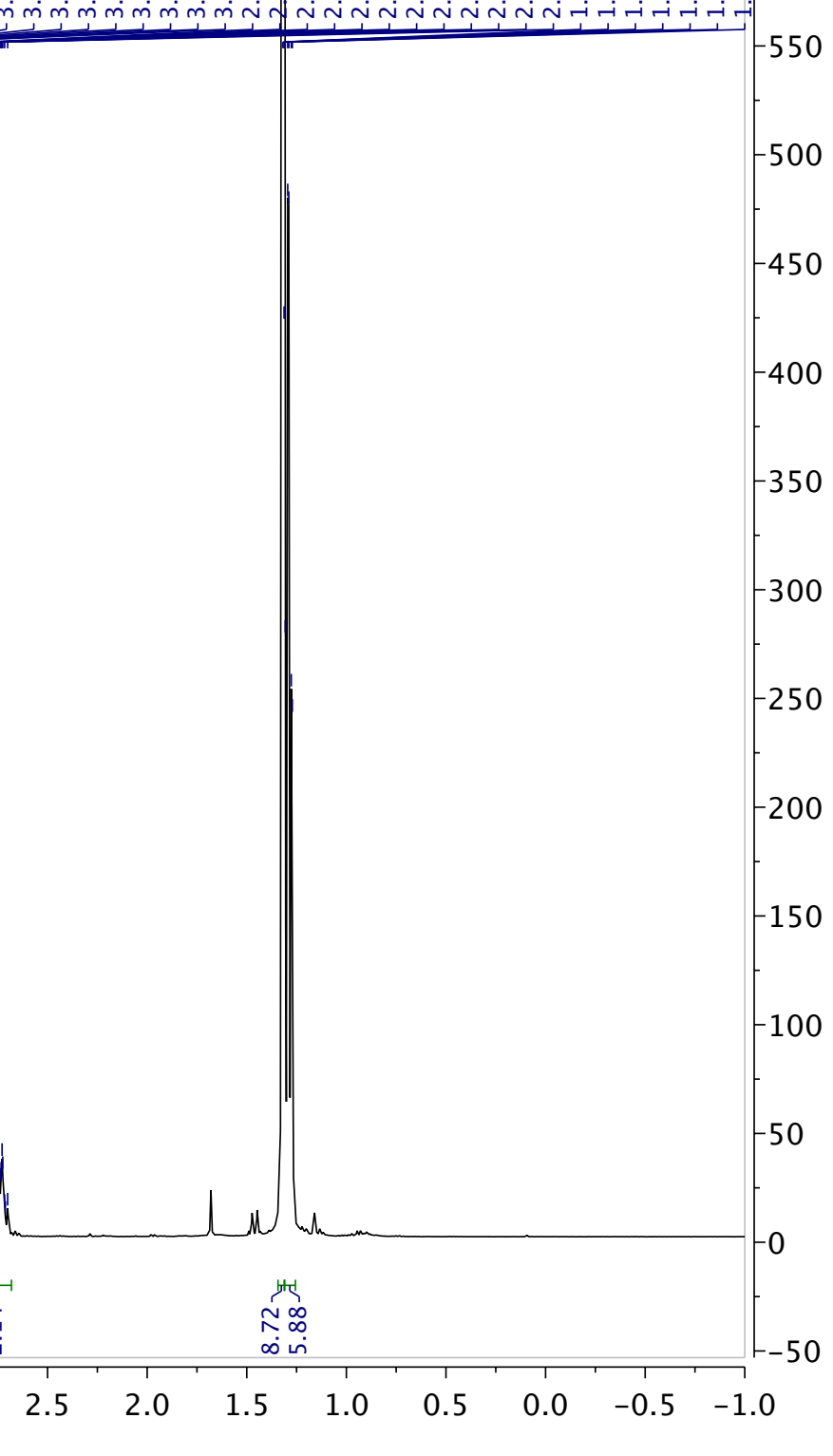




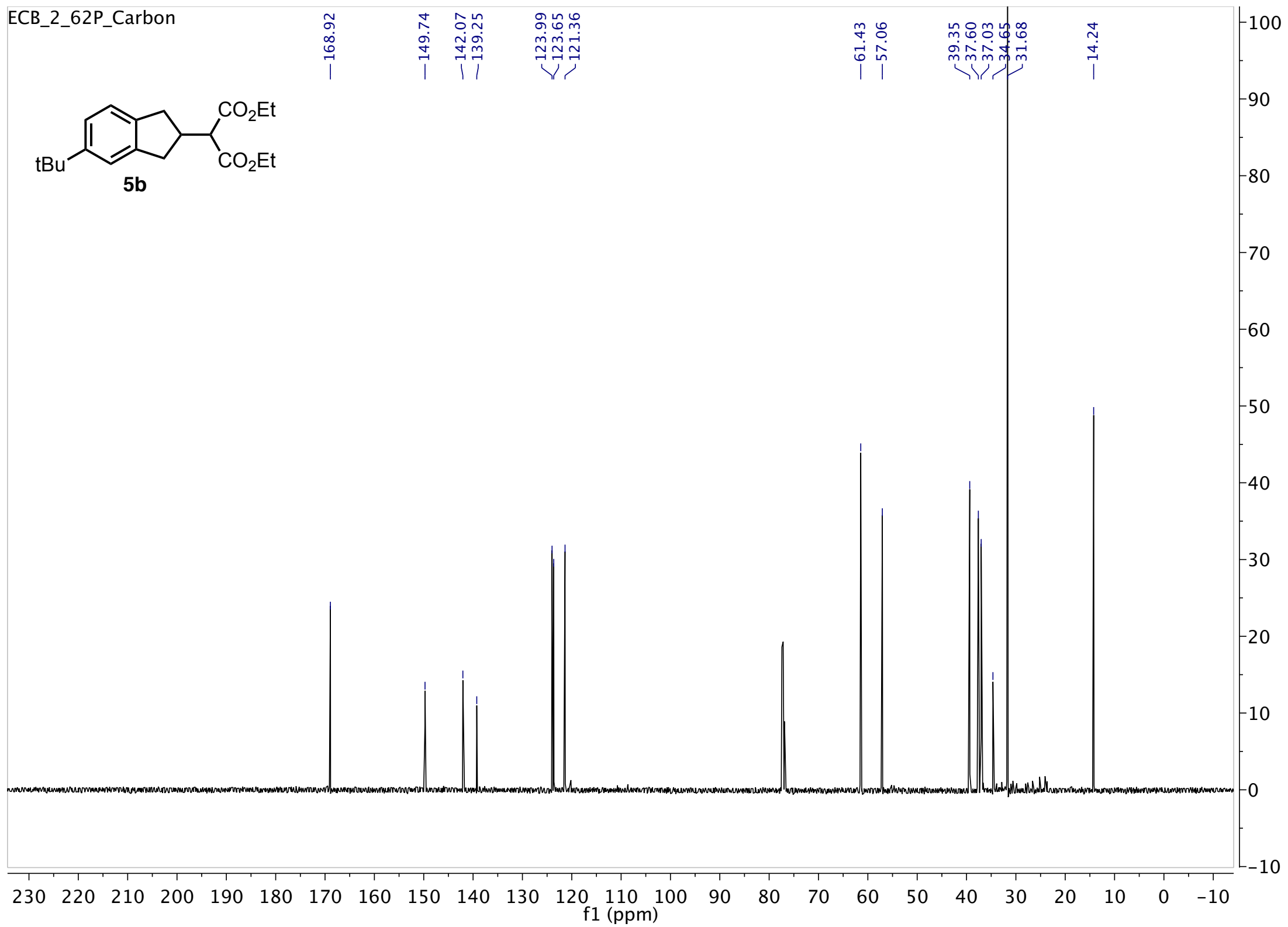




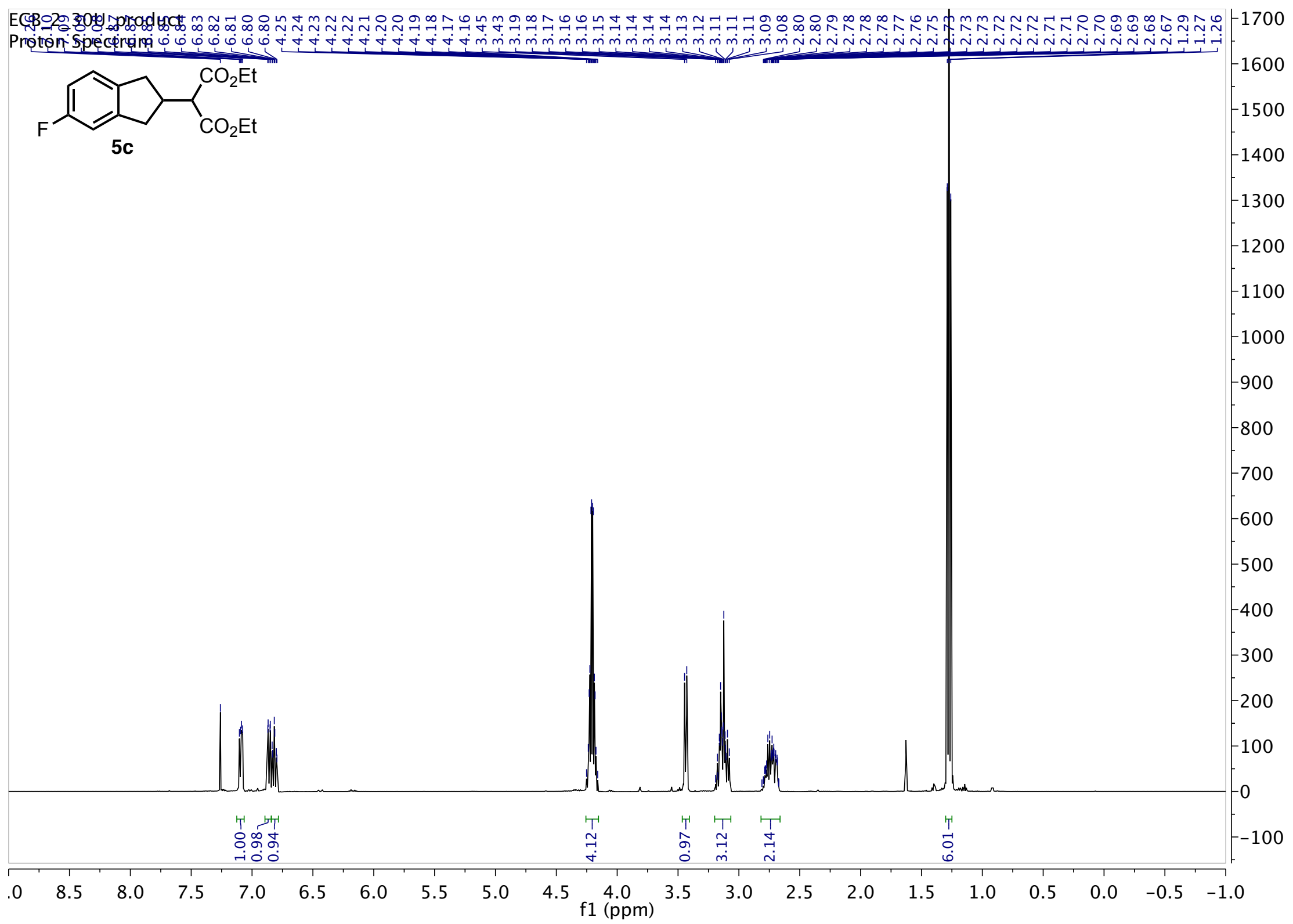




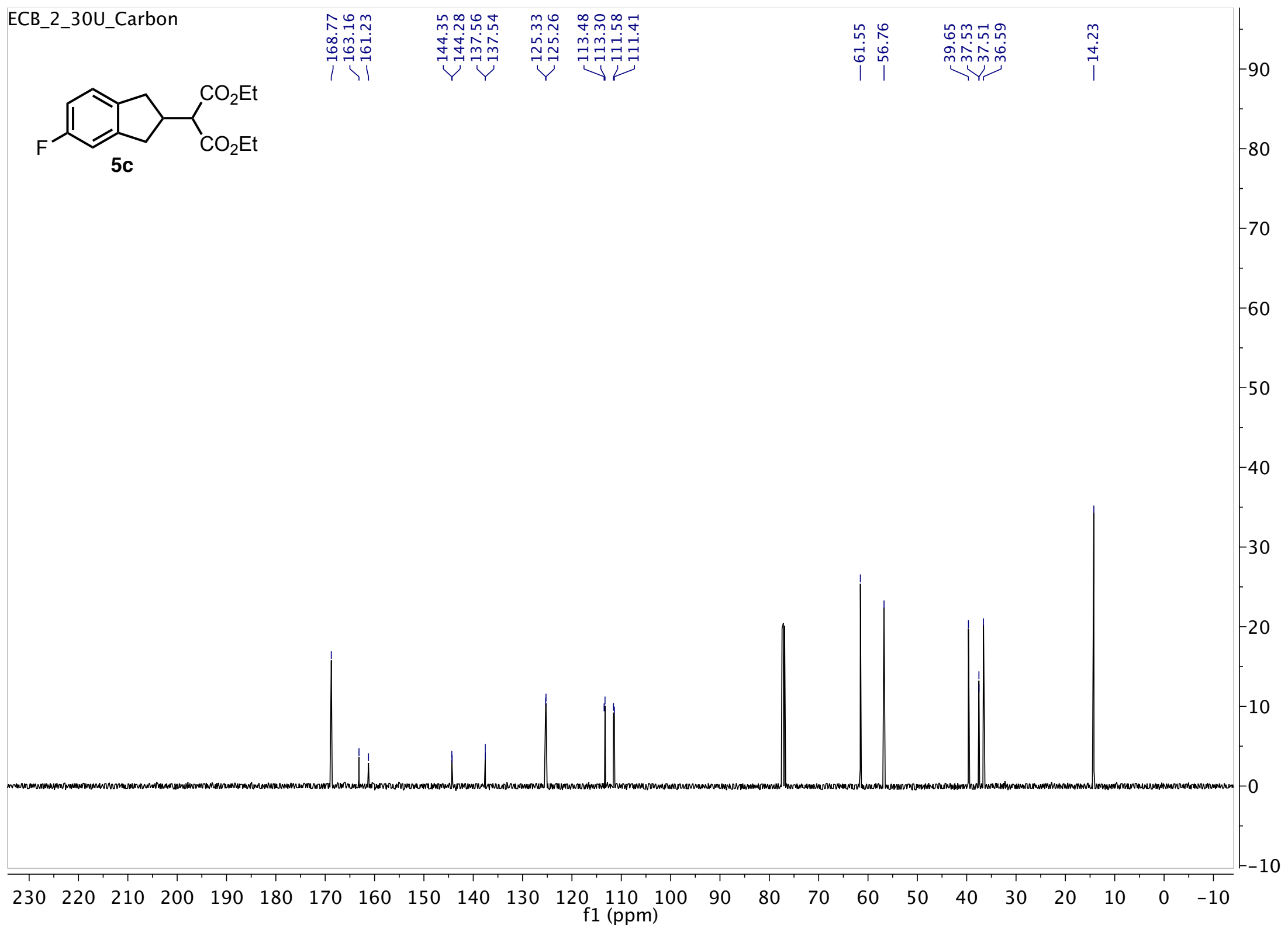




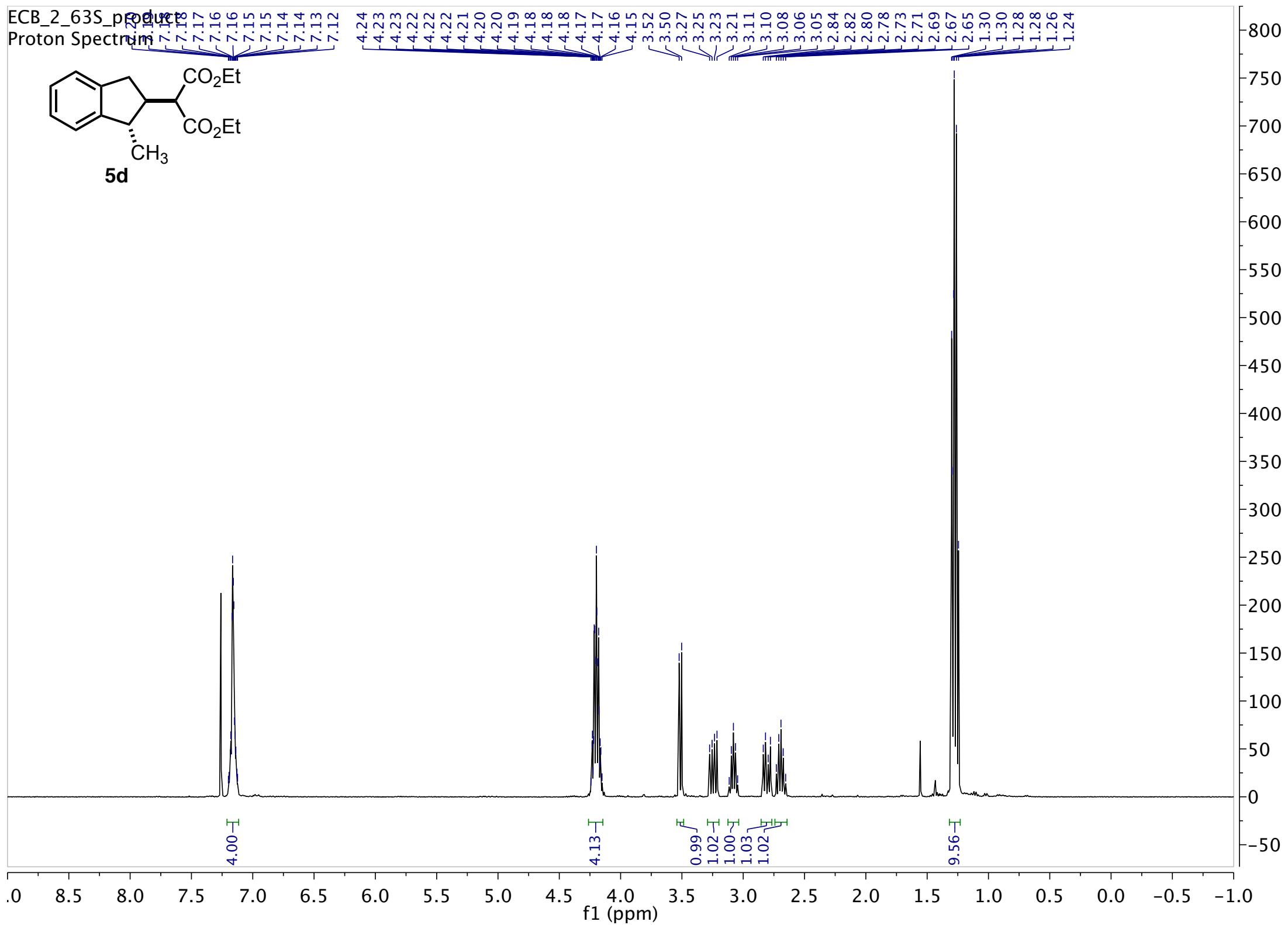




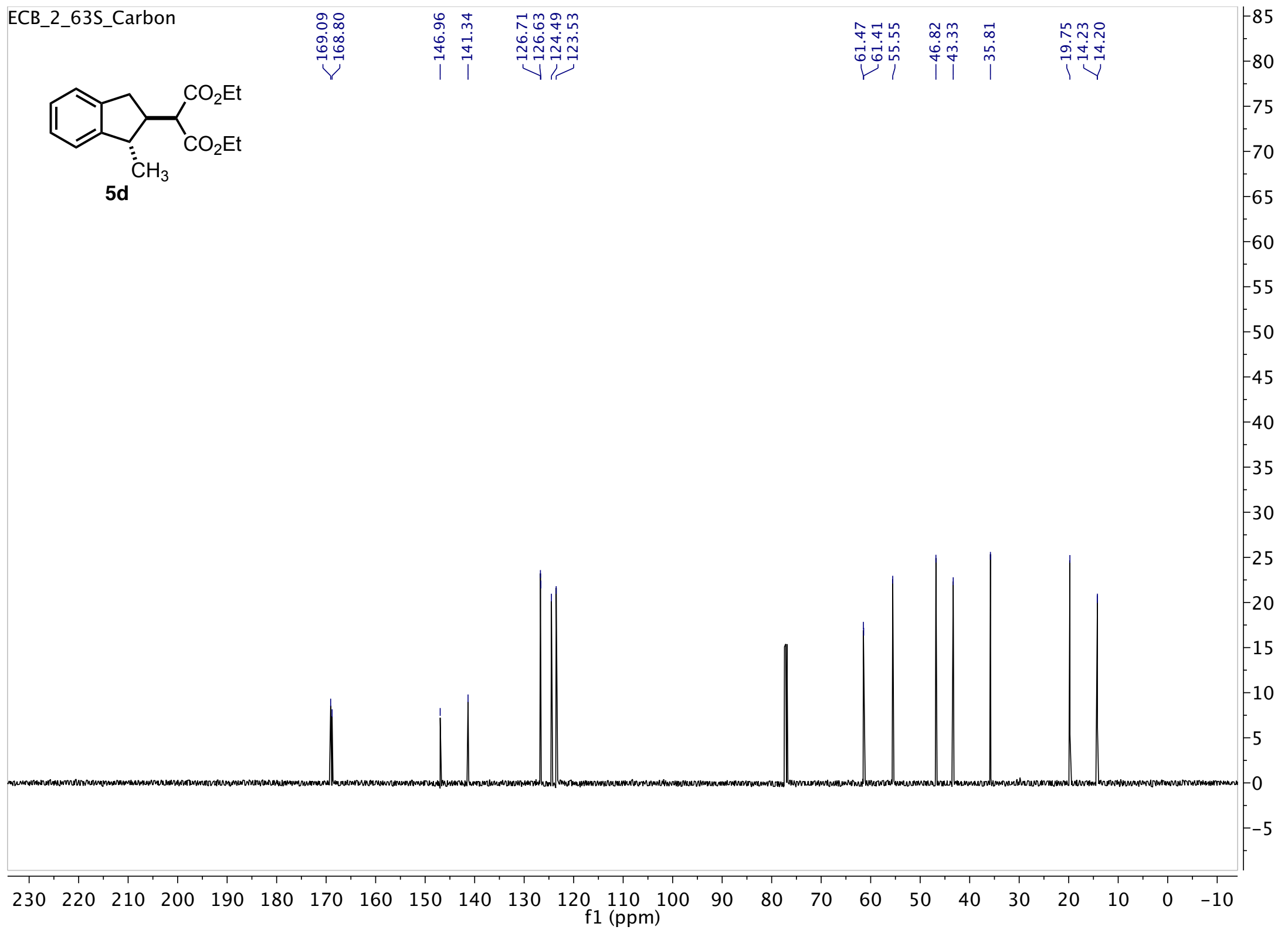




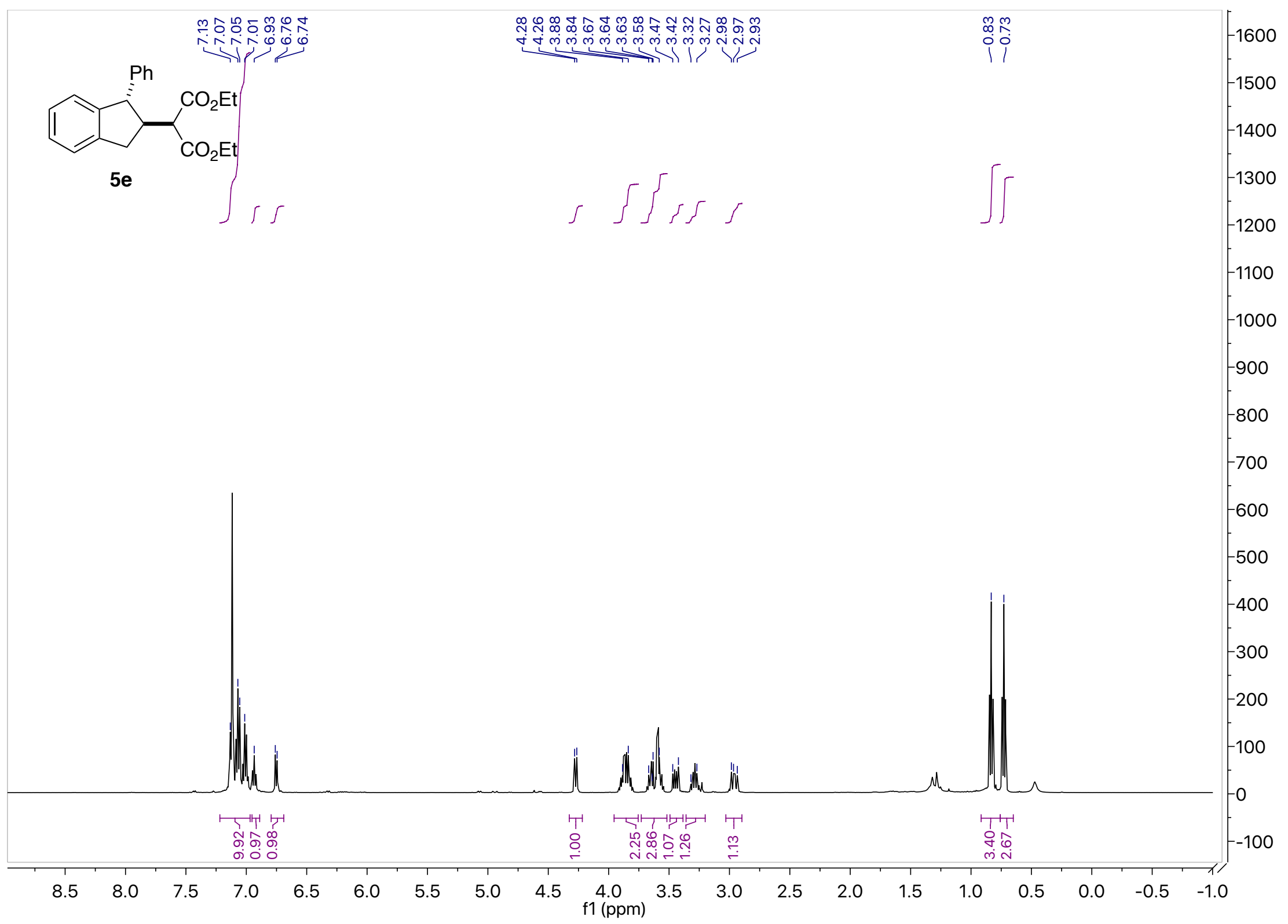




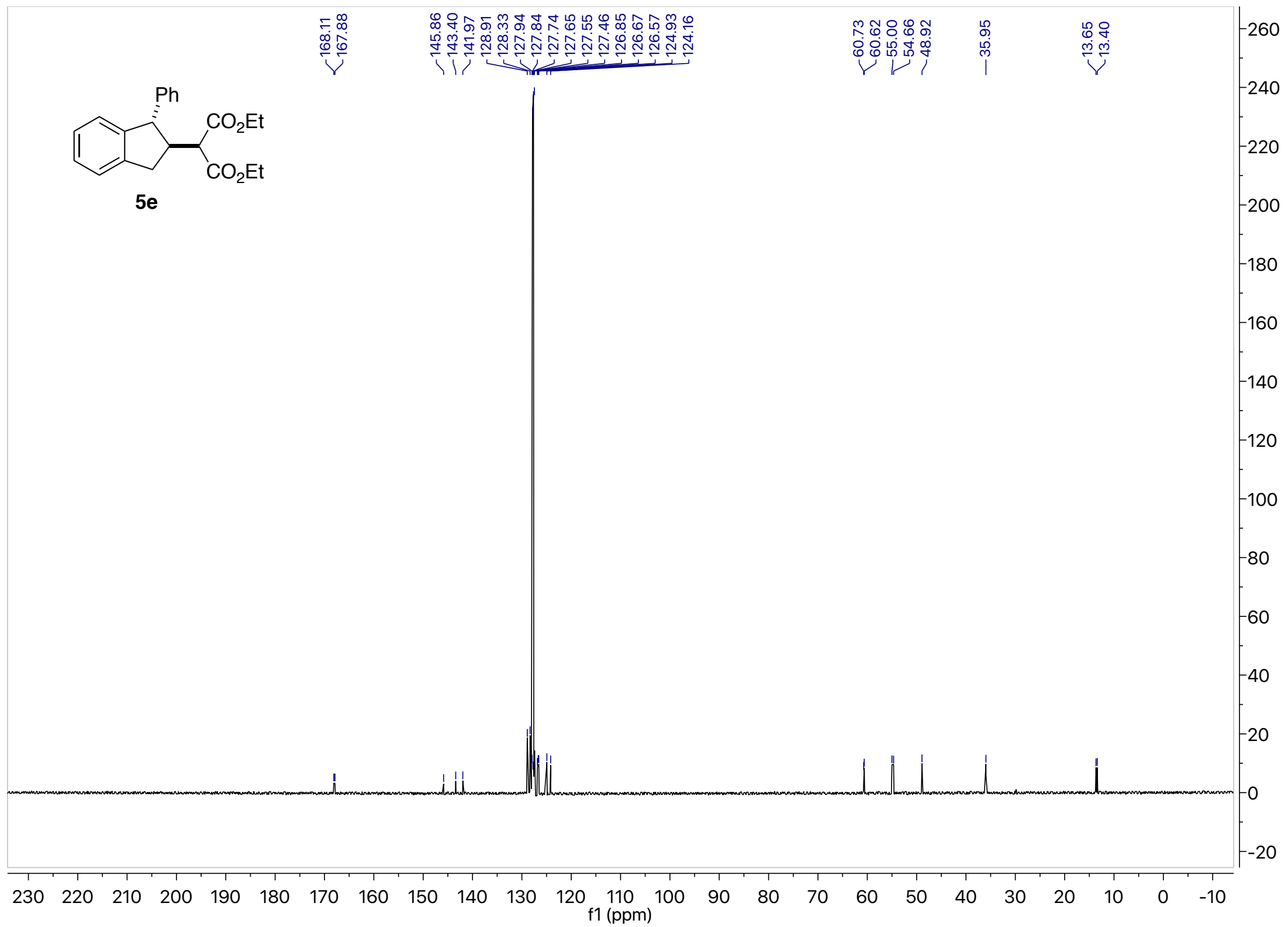




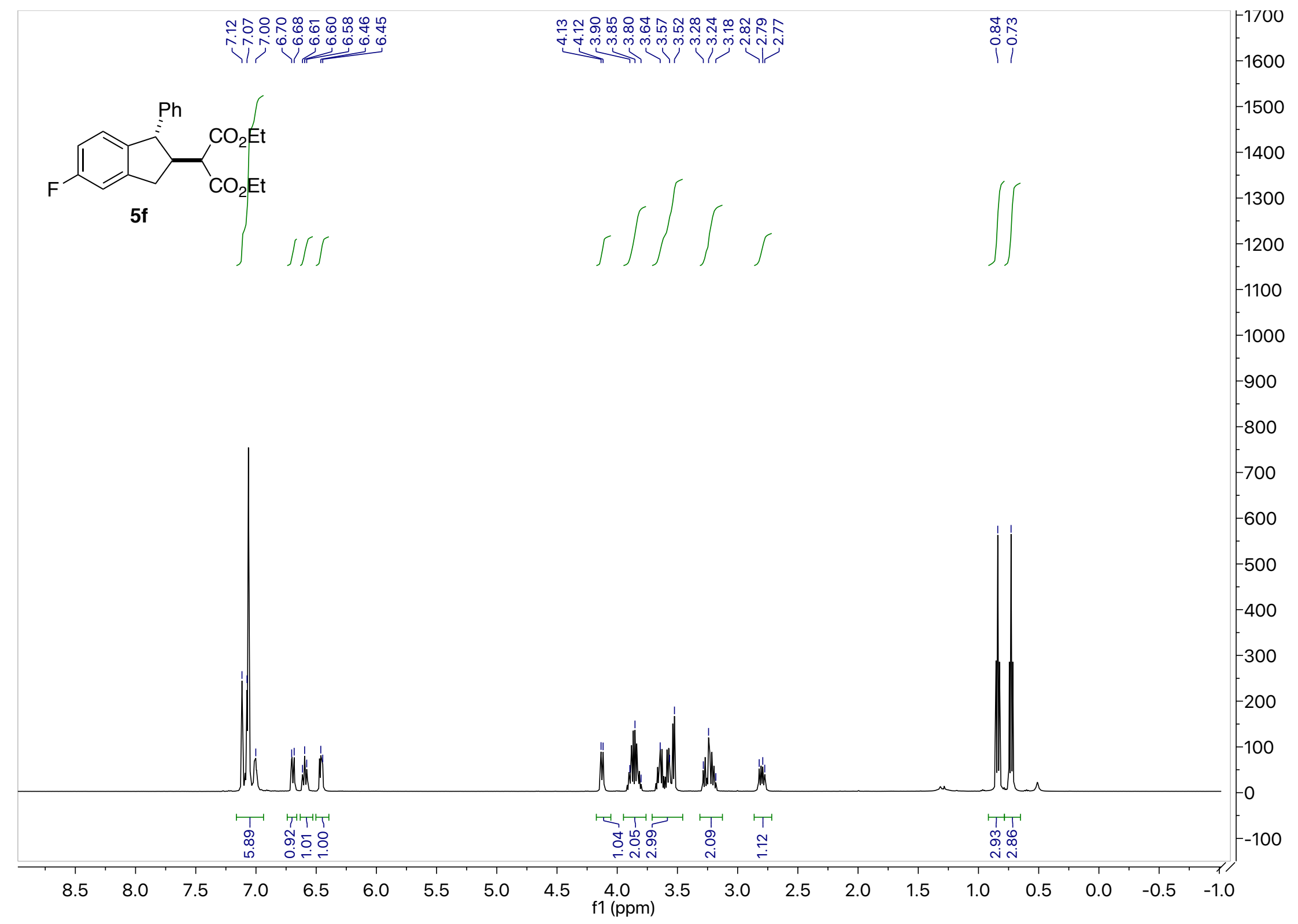




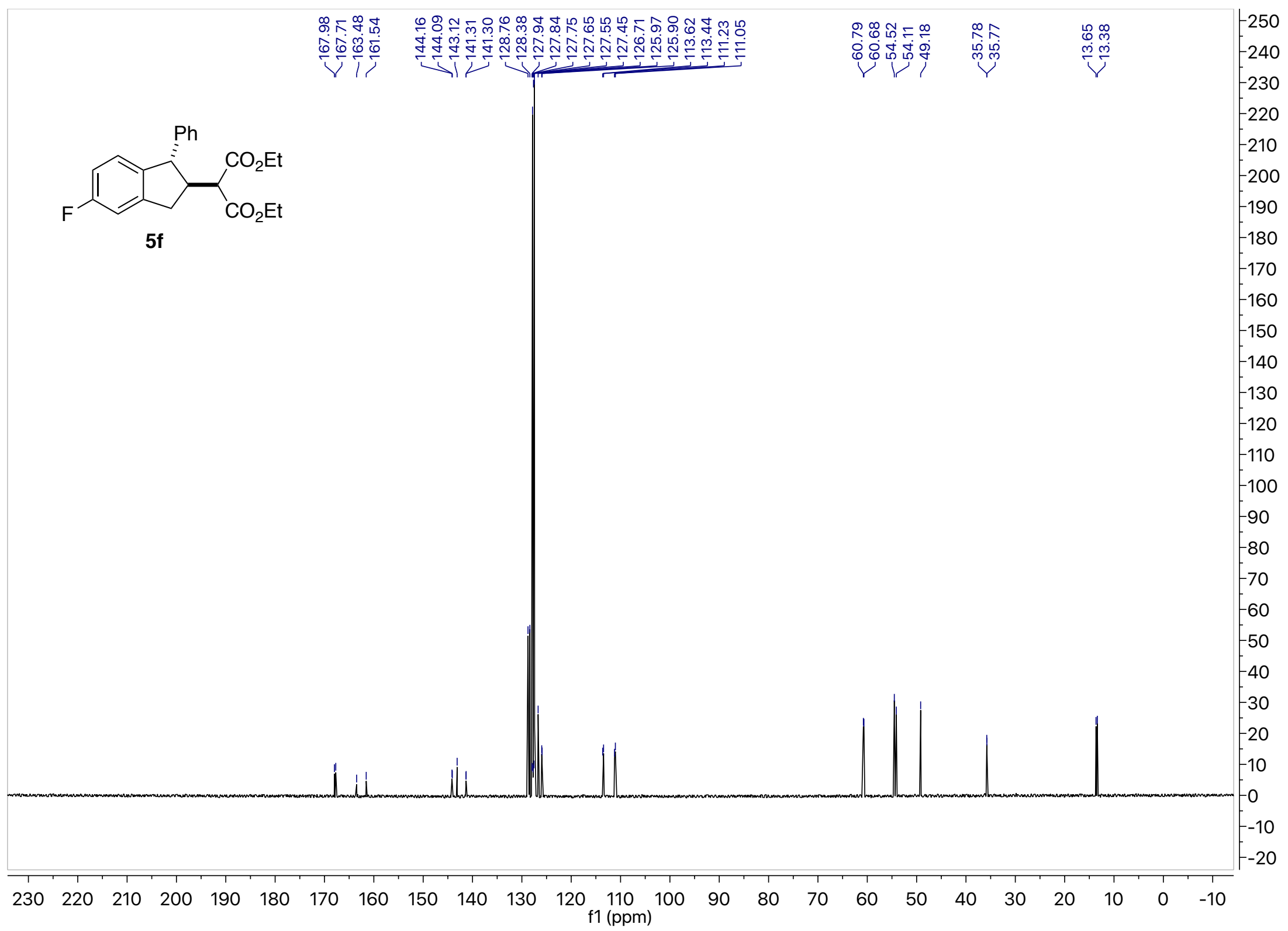




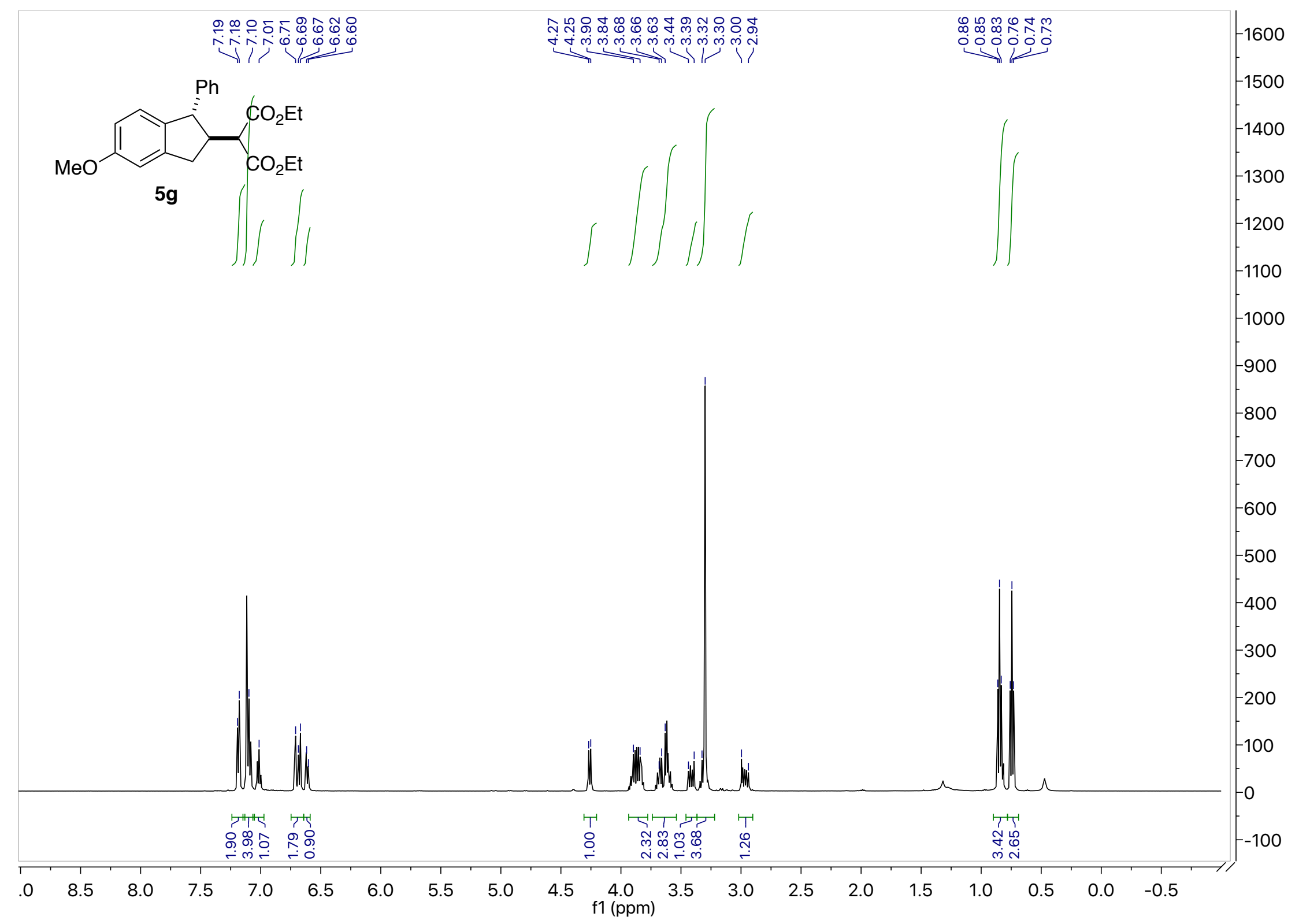




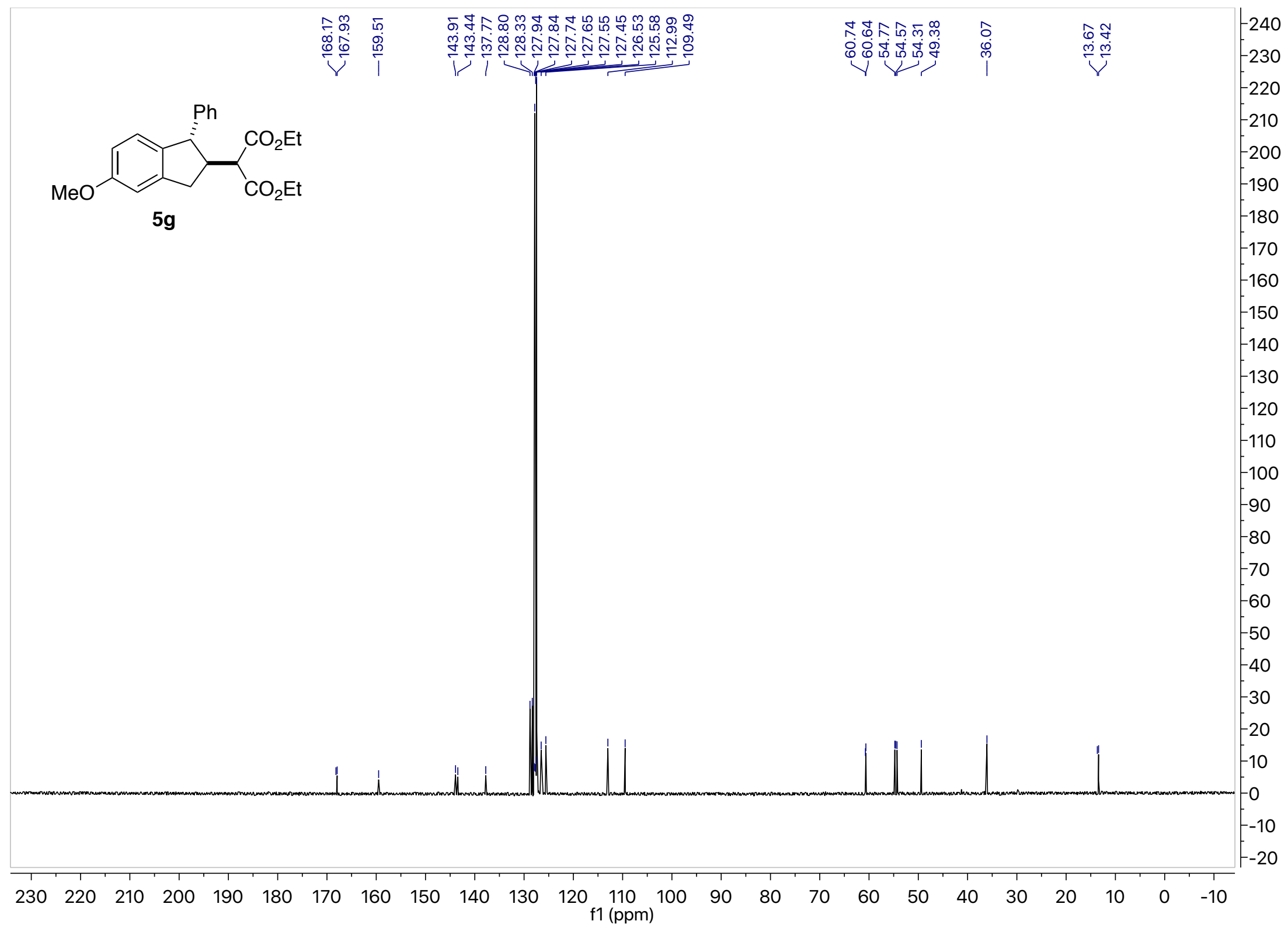




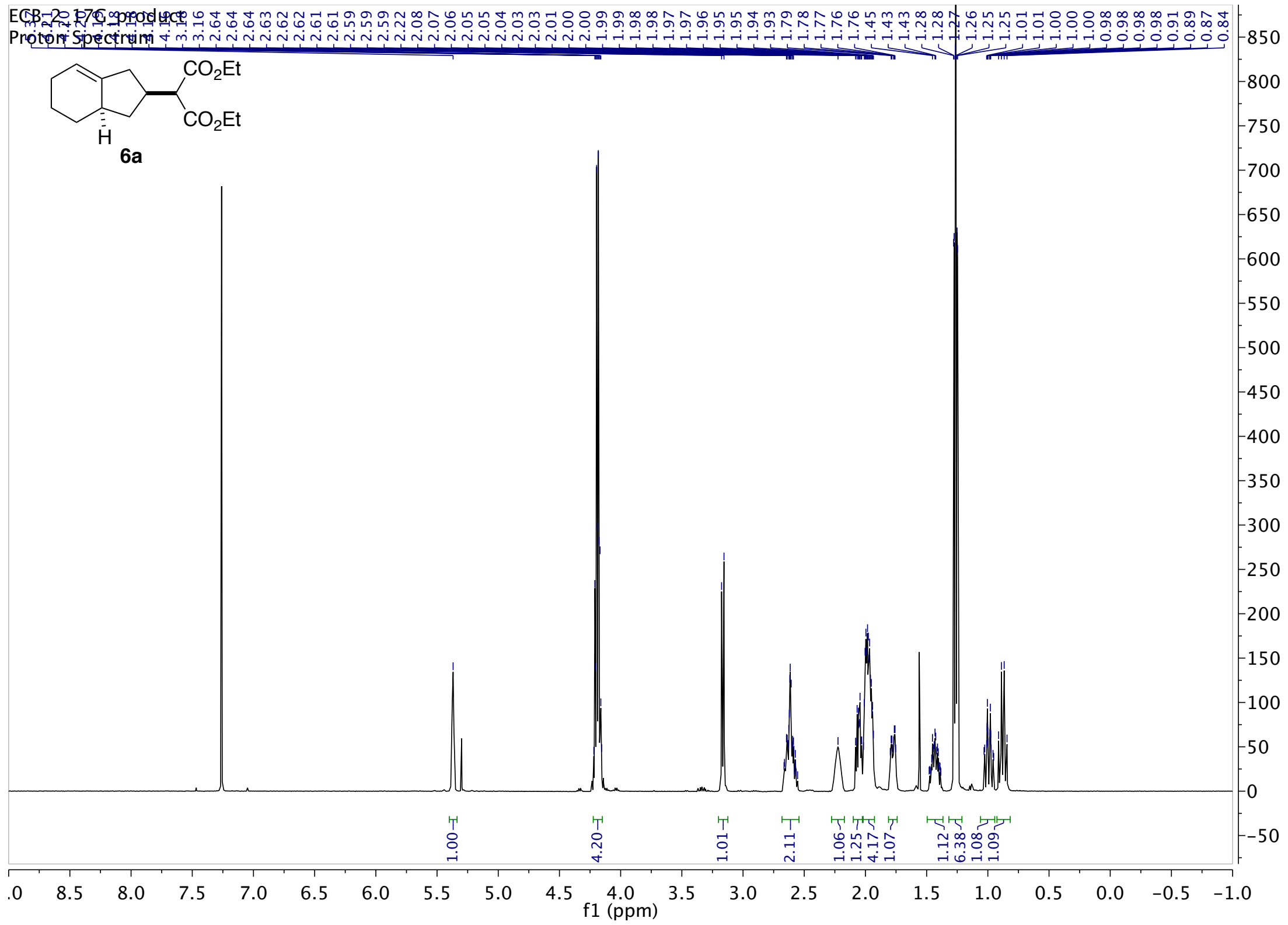



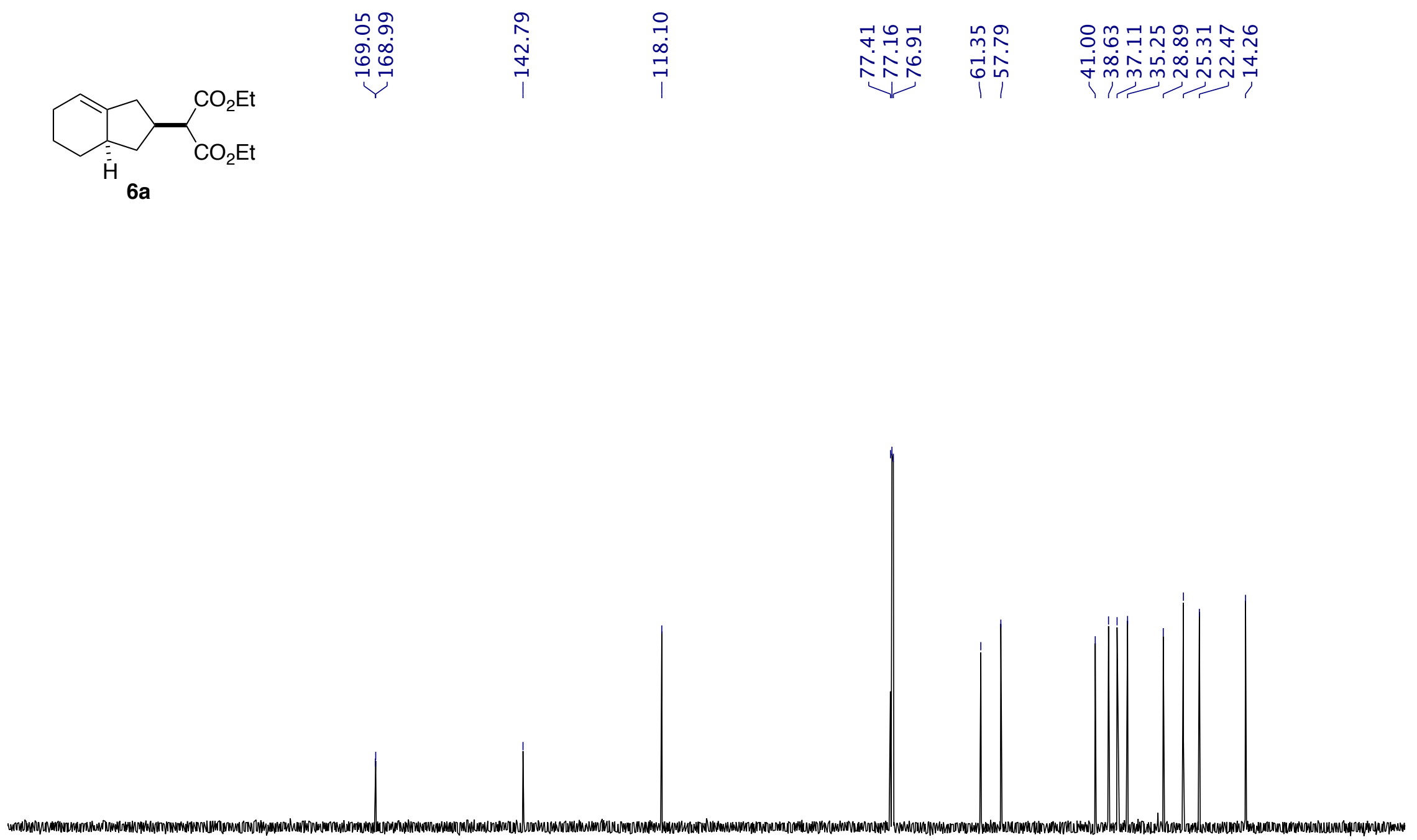

$\begin{array}{lllllllllllllllllllllllllllllll}230 & 220 & 210 & 200 & 190 & 180 & 170 & 160 & 150 & 140 & 130 & 120 & 110 & 100 & 90 & 80 & 70 & 60 & 50 & 40 & 30 & 20 & 10 & 0 & -10\end{array}$ 


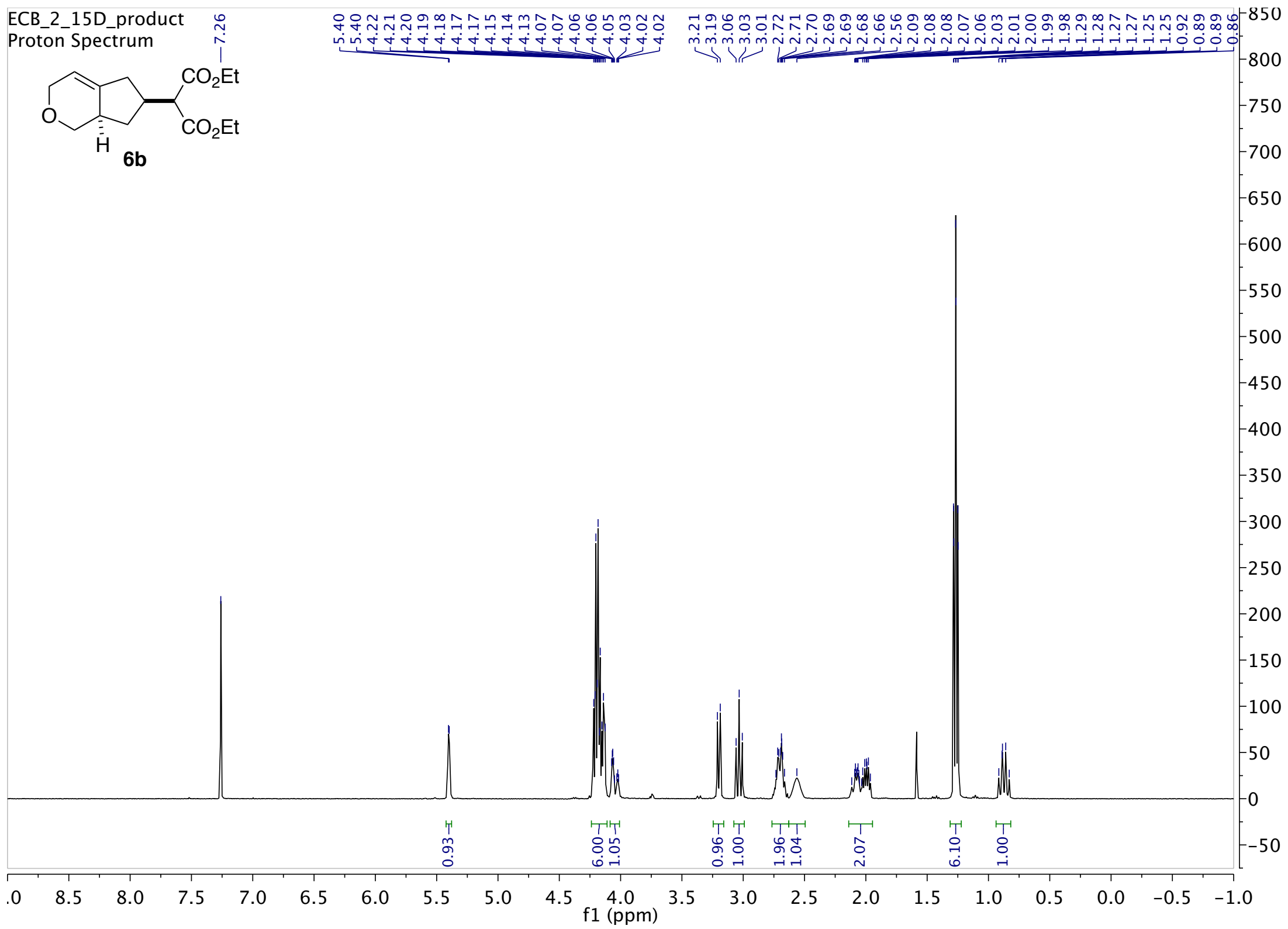




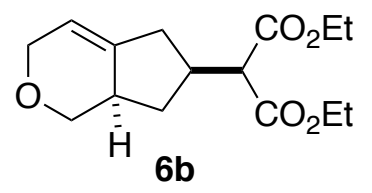

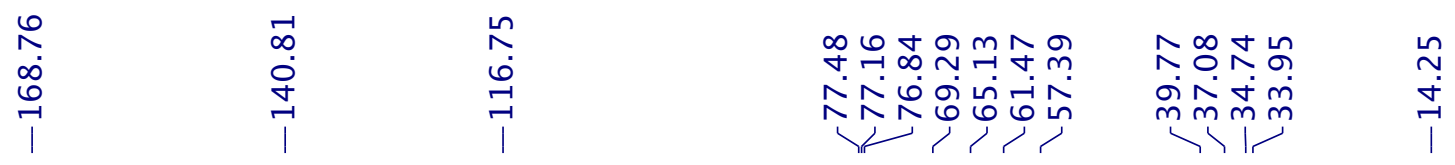

$6 b$

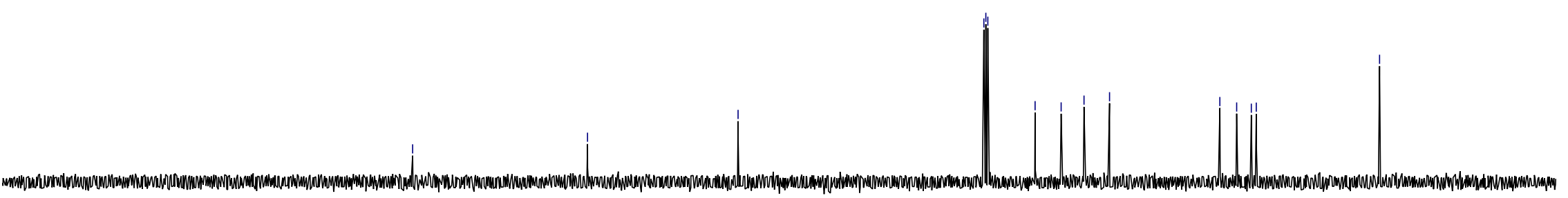
$\begin{array}{lllllllllllllllllllllllllllllllll}230 & 220 & 210 & 200 & 190 & 180 & 170 & 160 & 150 & 140 & 130 & 120 & 110 & 100 & 90 & 80 & 70 & 60 & 50 & 40 & 30 & 20 & 10 & 0 & -10\end{array}$ 


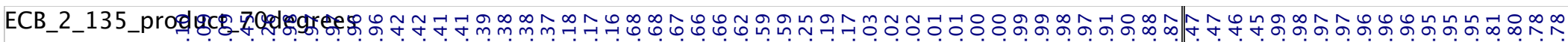
nं

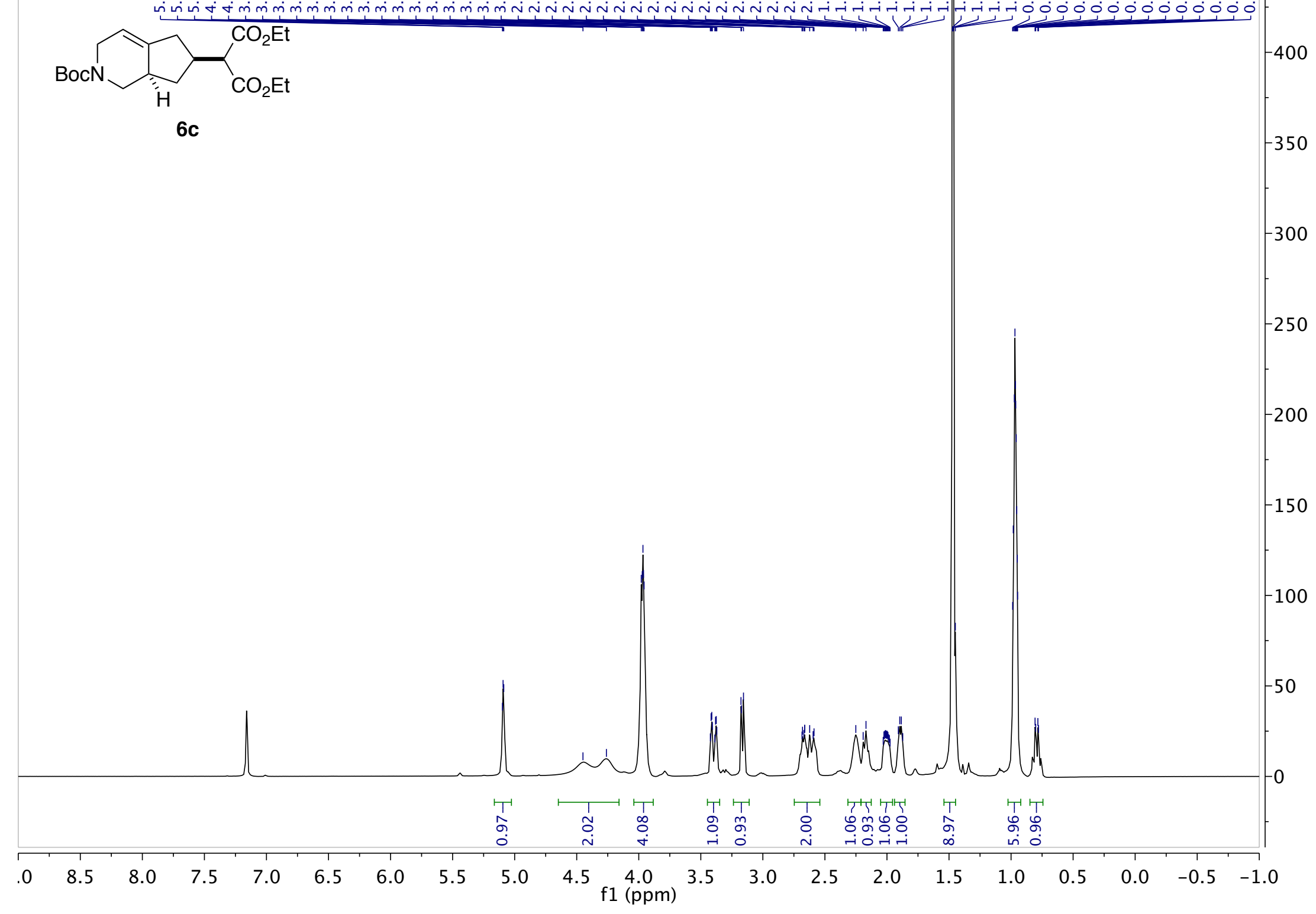




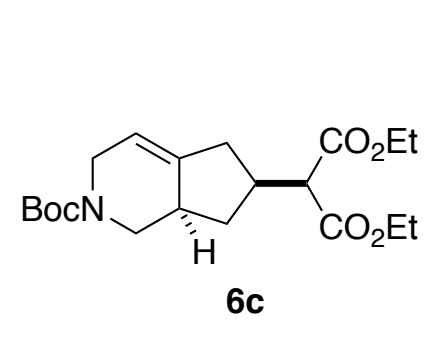

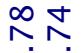

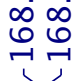

$\hat{6} \stackrel{\infty}{0} \div$

穴走定

祭

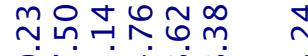

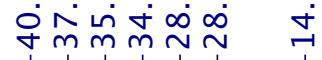

$6 c$

(1)

$\begin{array}{llllllllllllllllllllllllllllllllll}230 & 220 & 210 & 200 & 190 & 180 & 170 & 160 & 150 & 140 & 130 & 120 & 110 & 100 & 90 & 80 & 70 & 60 & 50 & 40 & 30 & 20 & 10 & 0 & -10\end{array}$ 


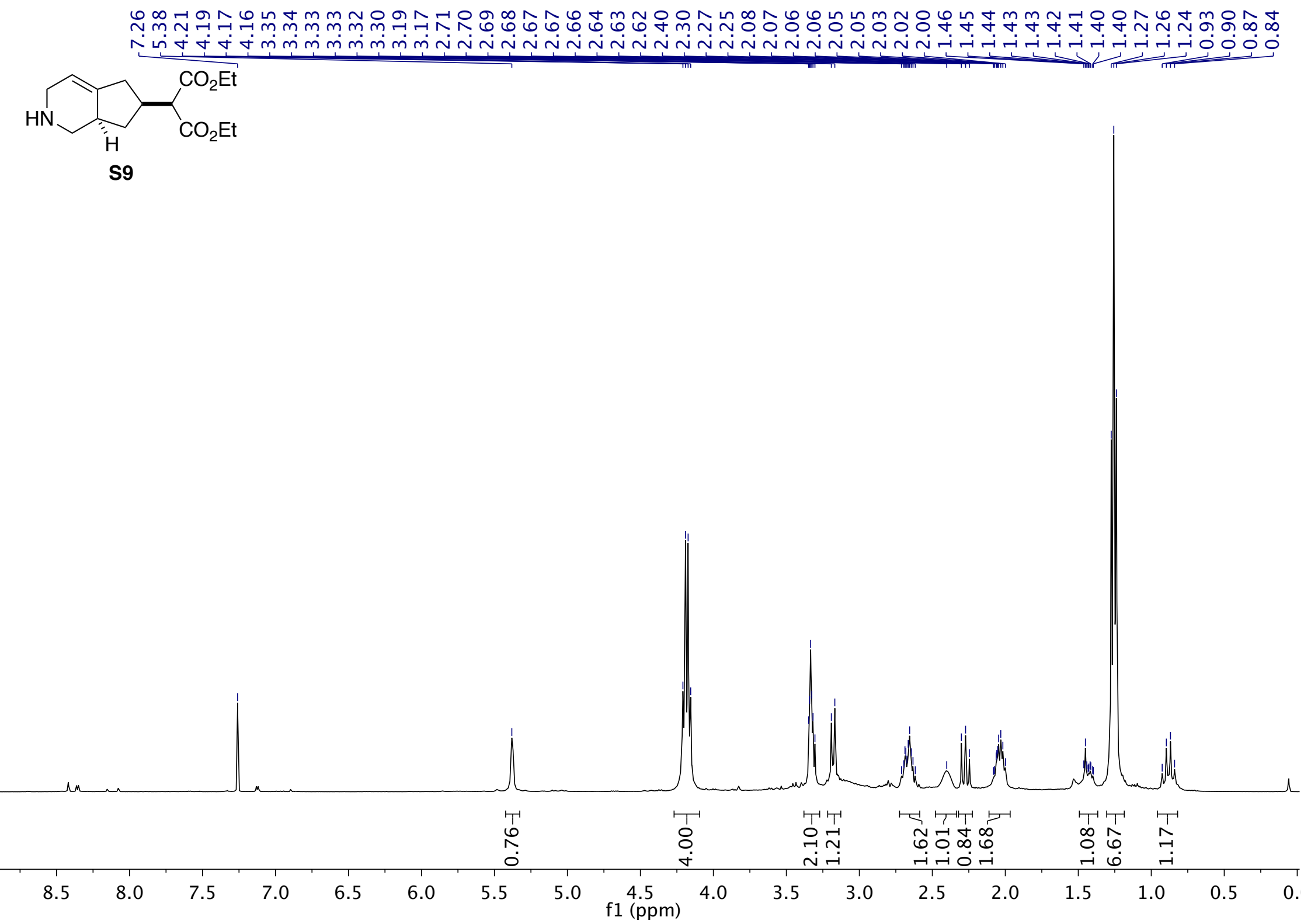




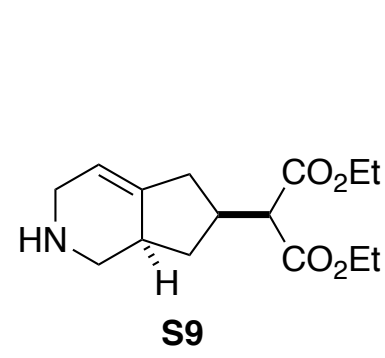

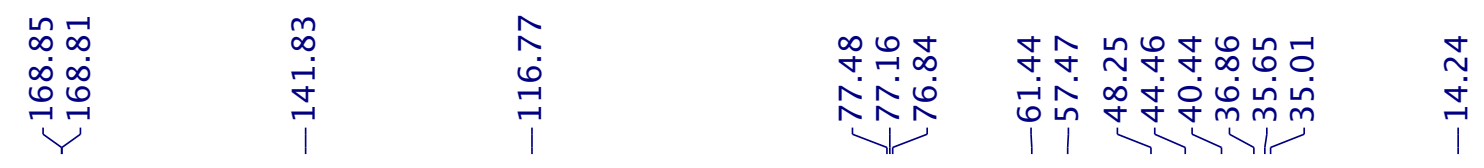

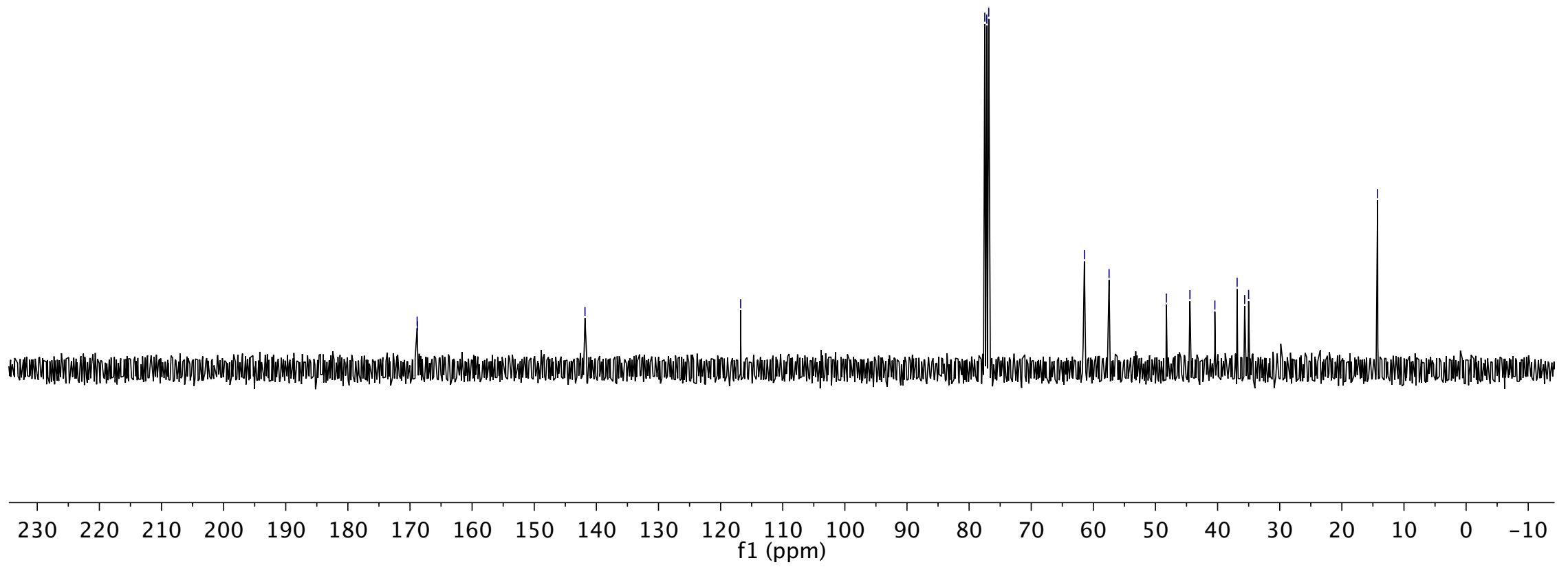




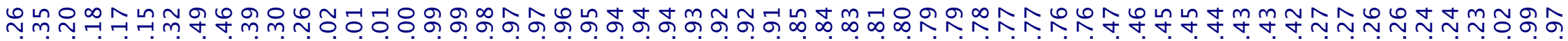
Nu

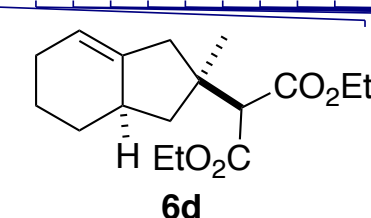

6d

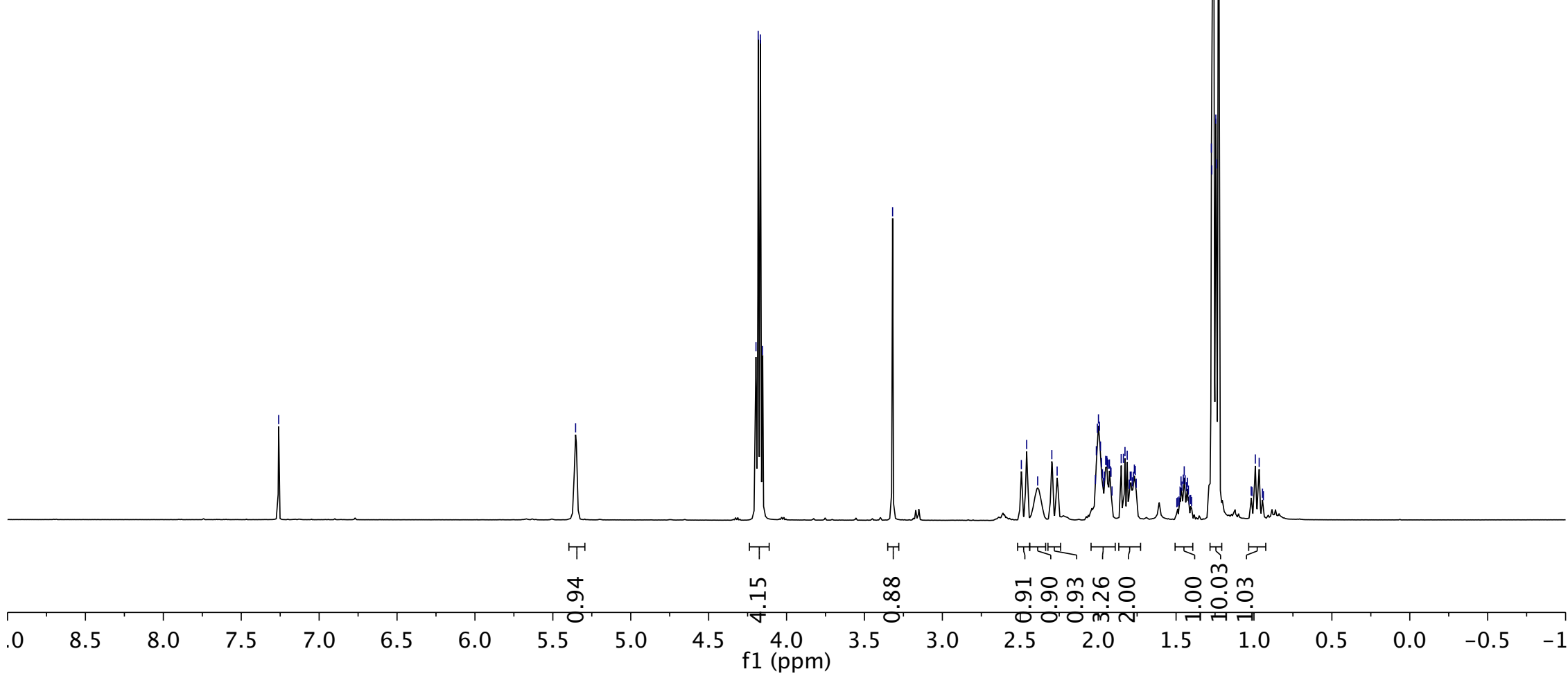



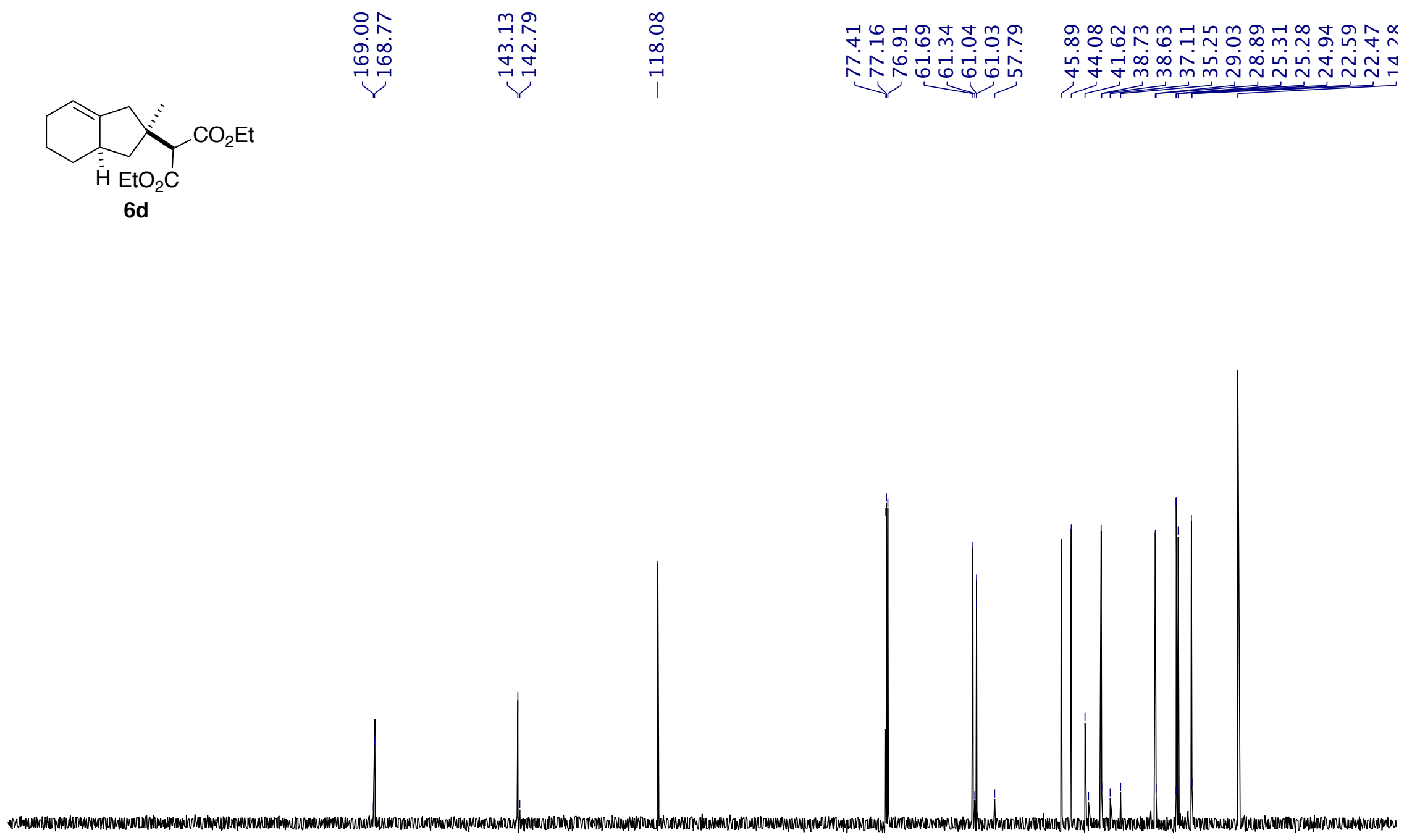

2302

$\begin{array}{lll}150 & 140\end{array}$

$\begin{array}{lllll}130 & 120 & 110 & 100\end{array}$

f1 (ppm)

$90 \quad 80$

70

60

50

403

$30 \quad 20$

10

$-10$ 


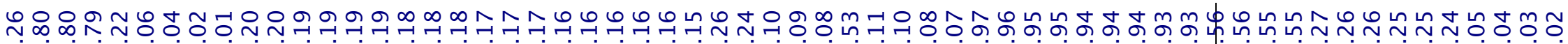
ベ ம்

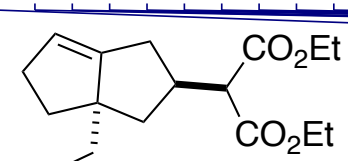

$6 e$

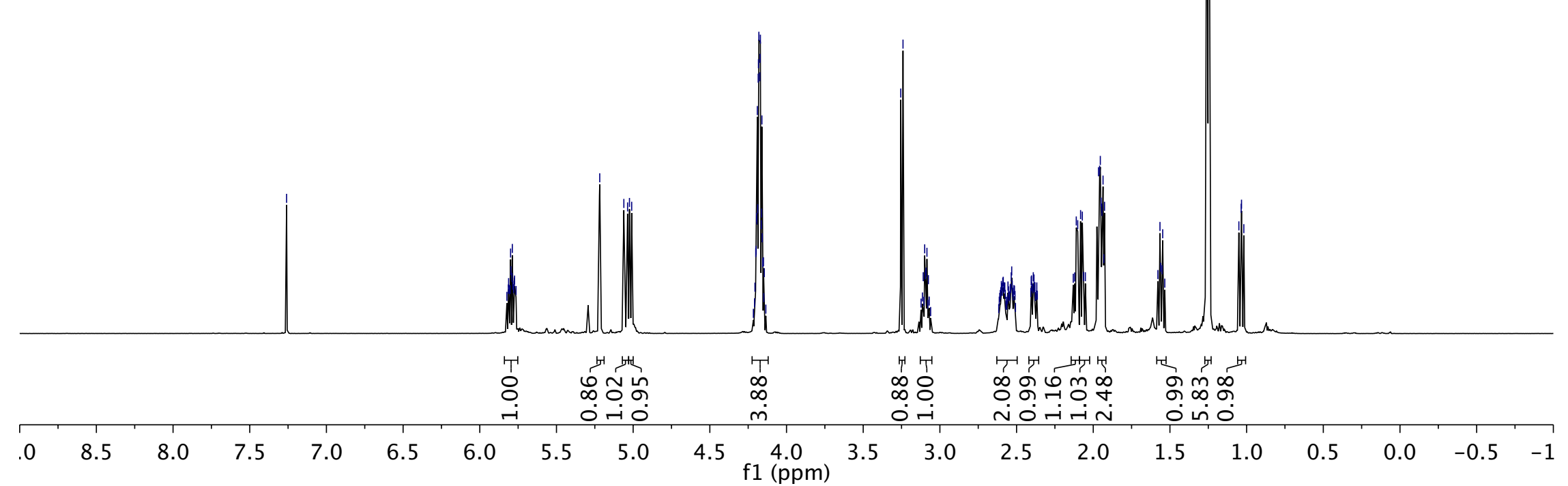



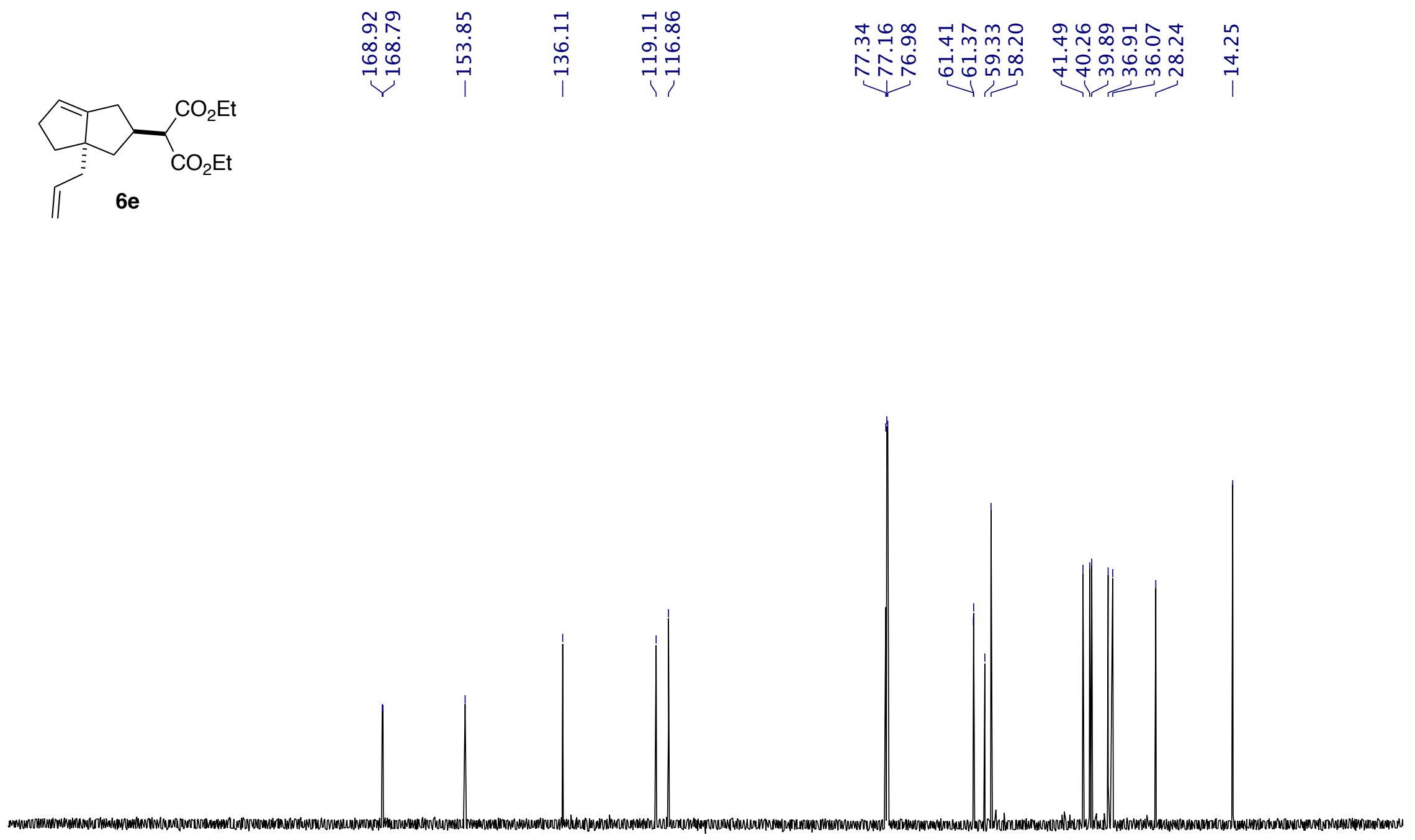

$\begin{array}{llllllllllllllllllllllllllllll}230 & 220 & 210 & 200 & 190 & 180 & 170 & 160 & 150 & 140 & 130 & 120 & 110 & 100 & 90 & 80 & 70 & 60 & 50 & 40 & 30 & 20 & 10 & 0 & -10\end{array}$ 


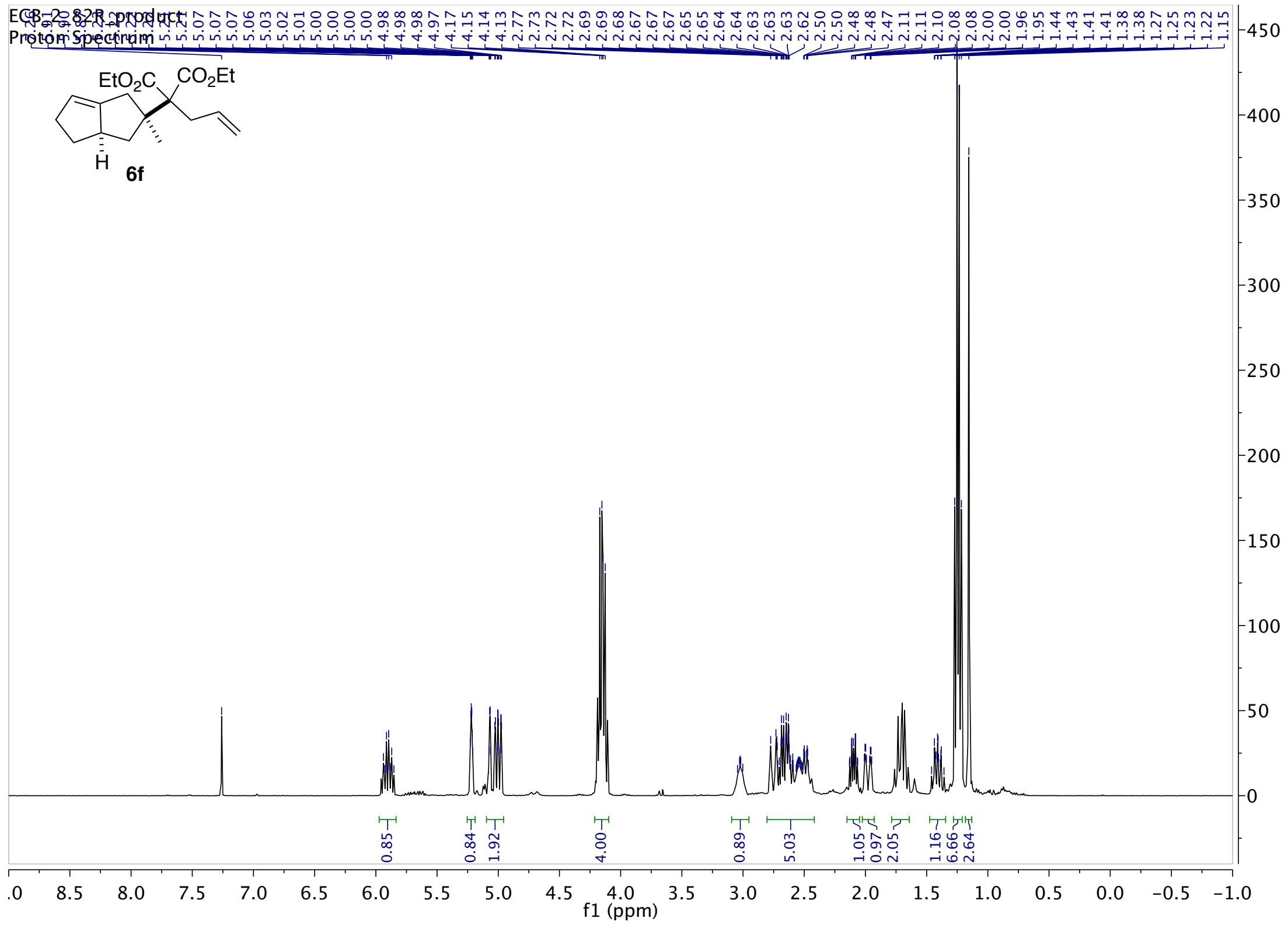




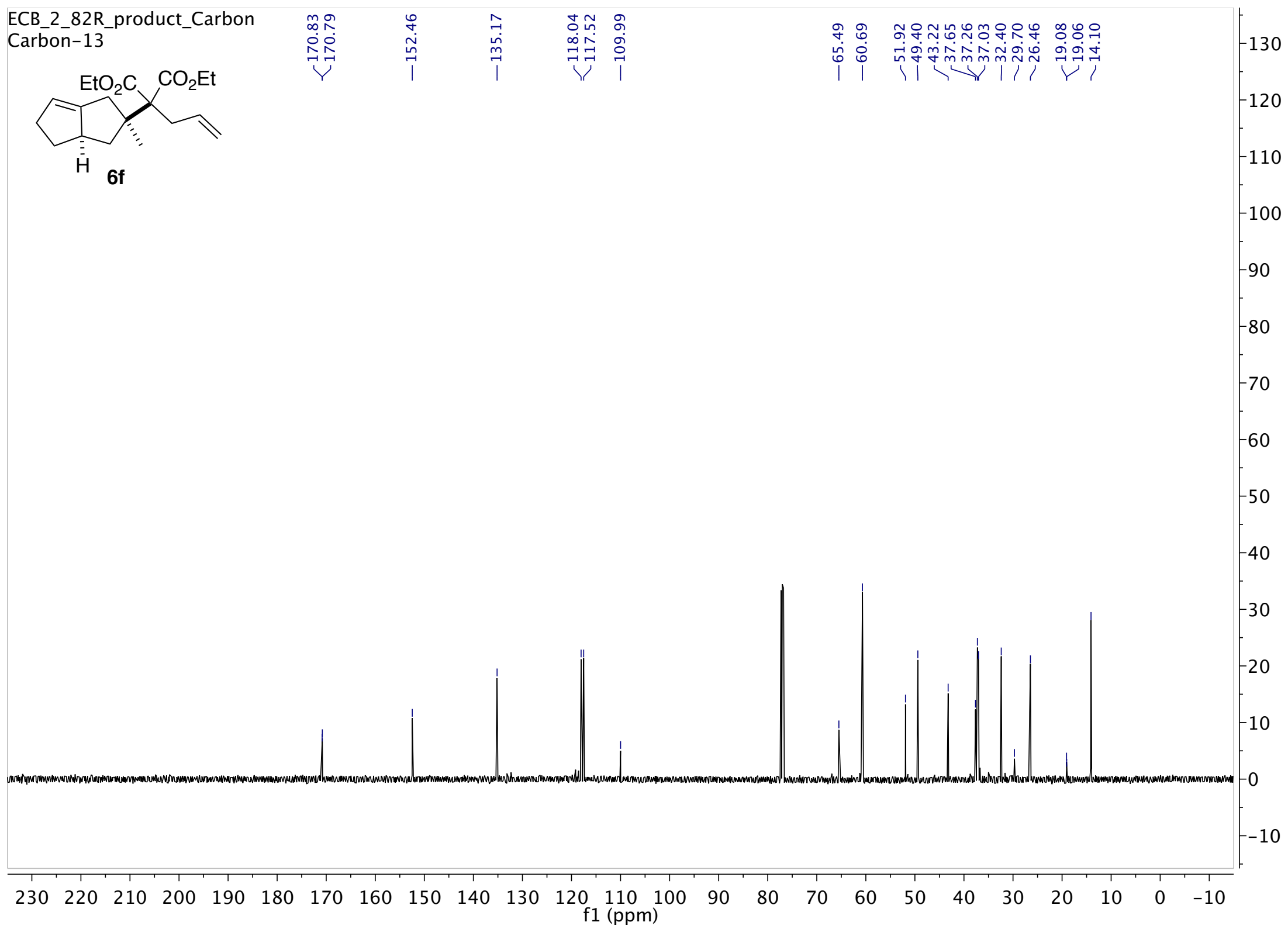




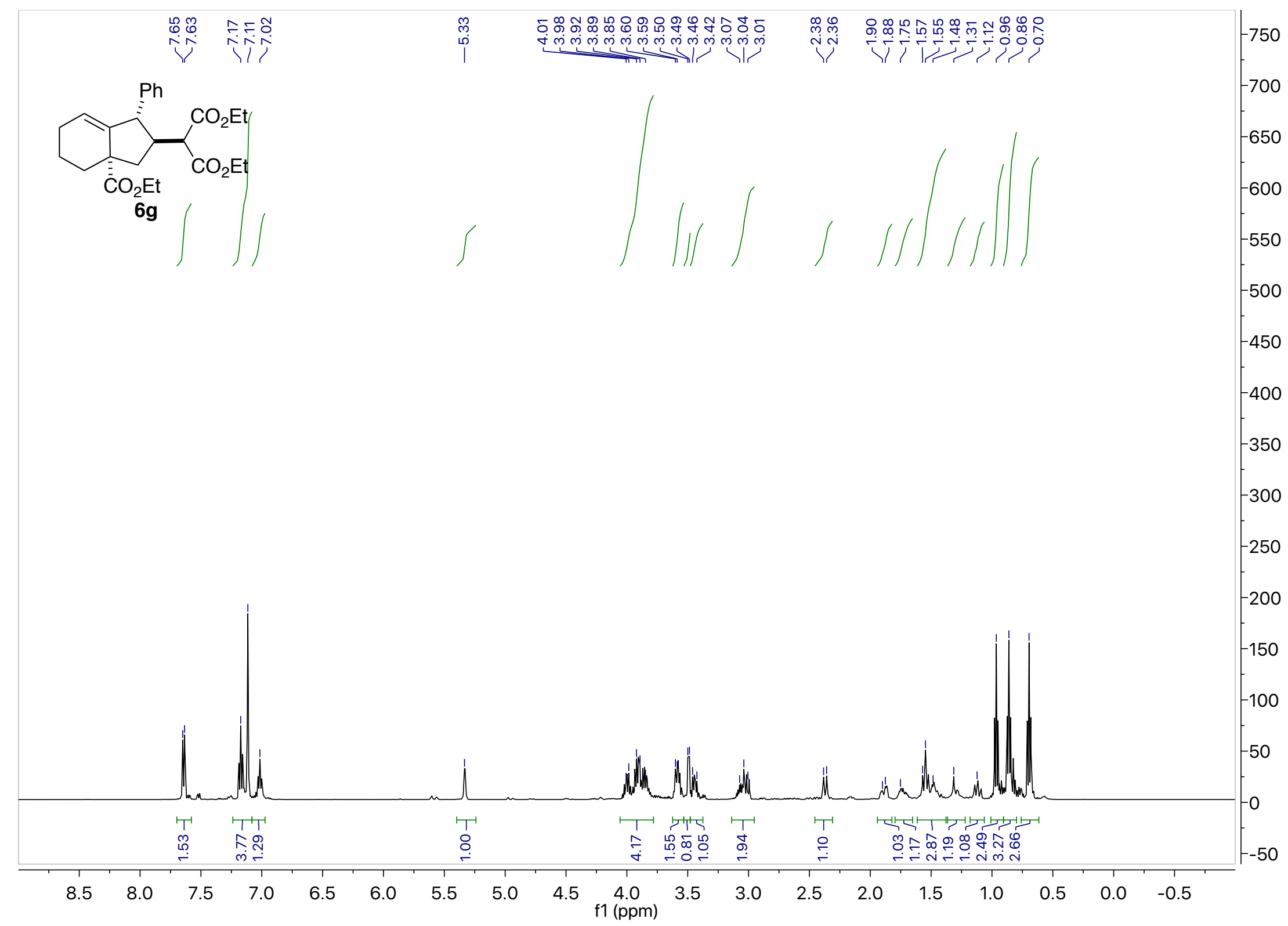




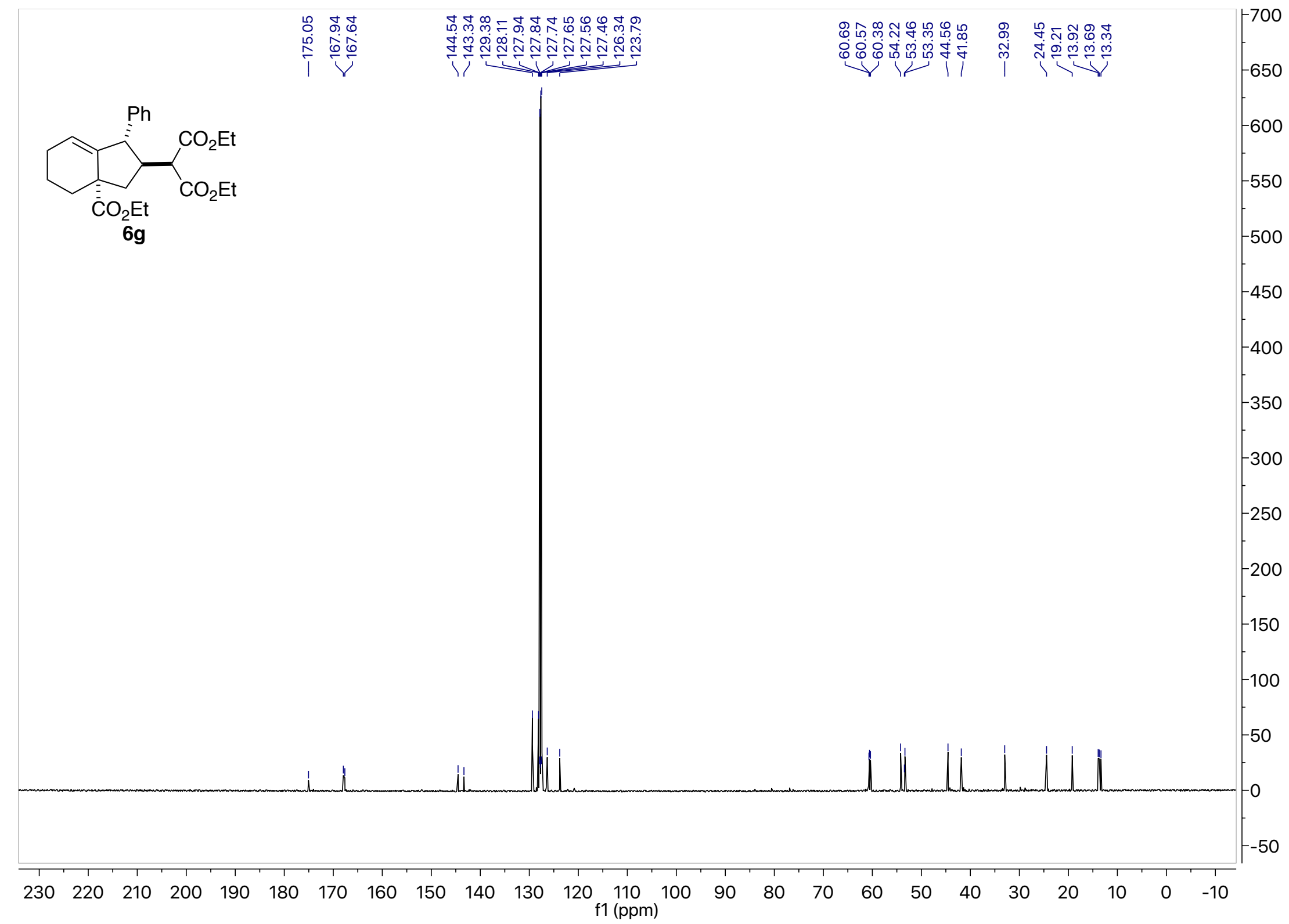




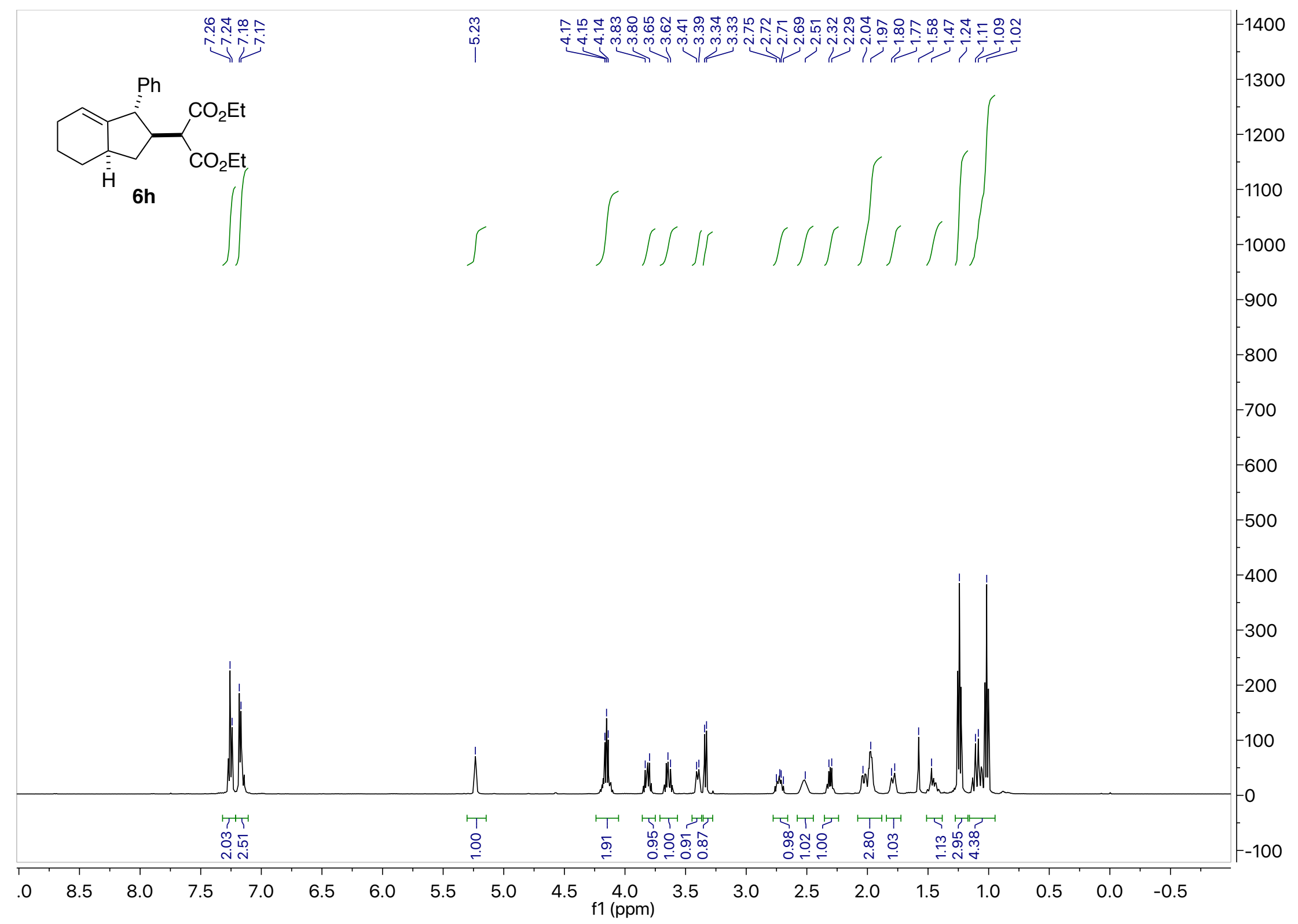




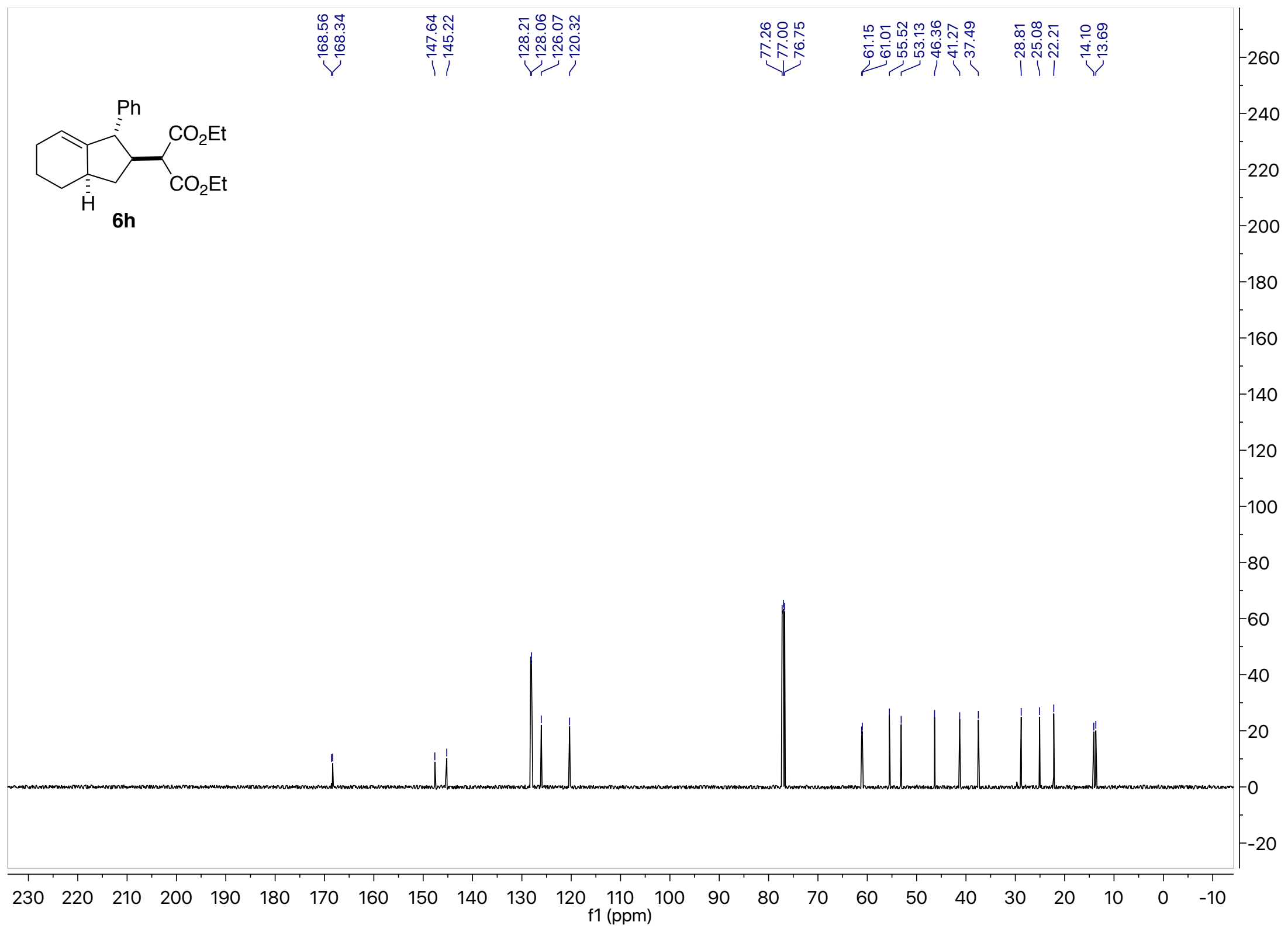




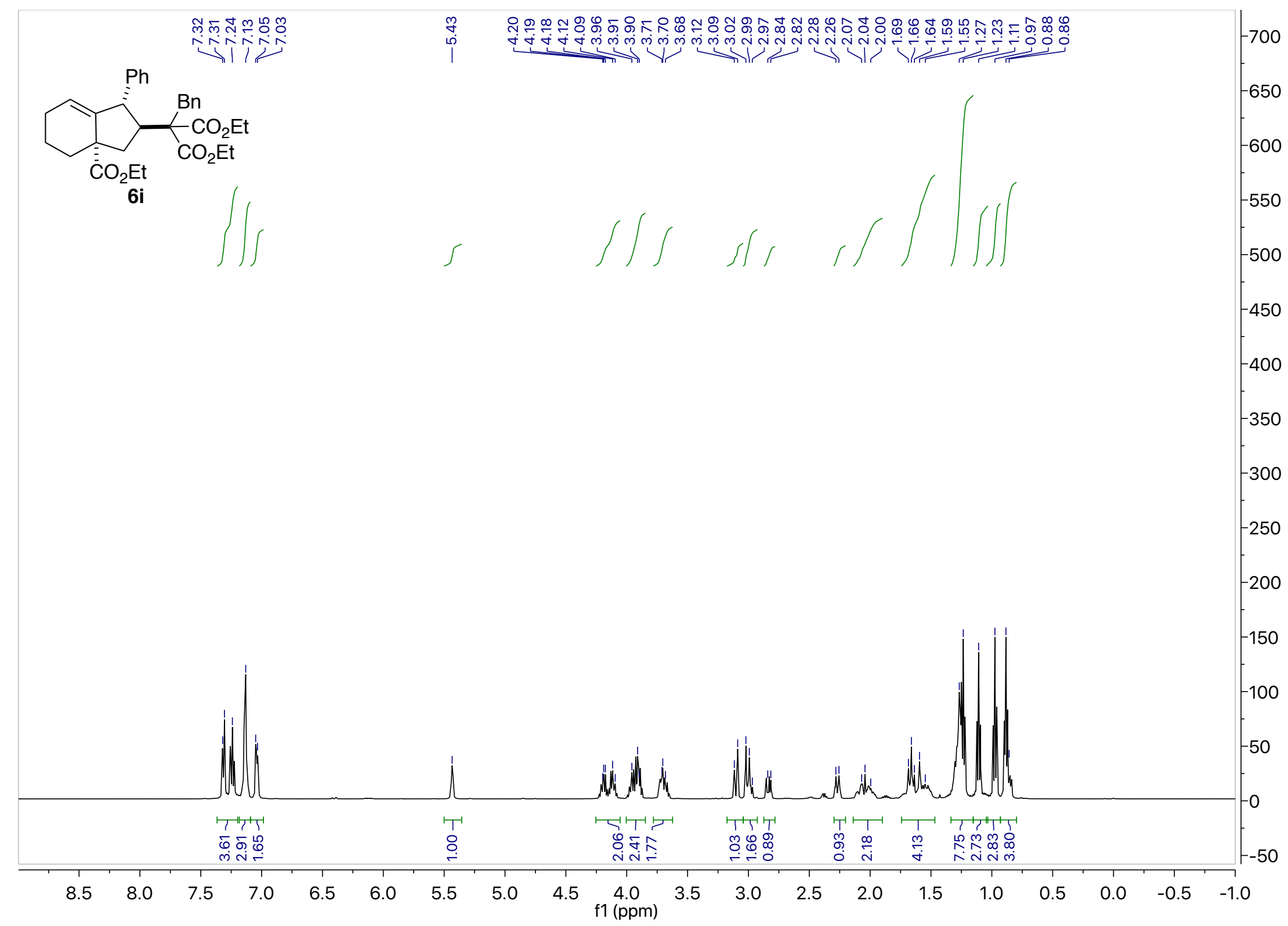




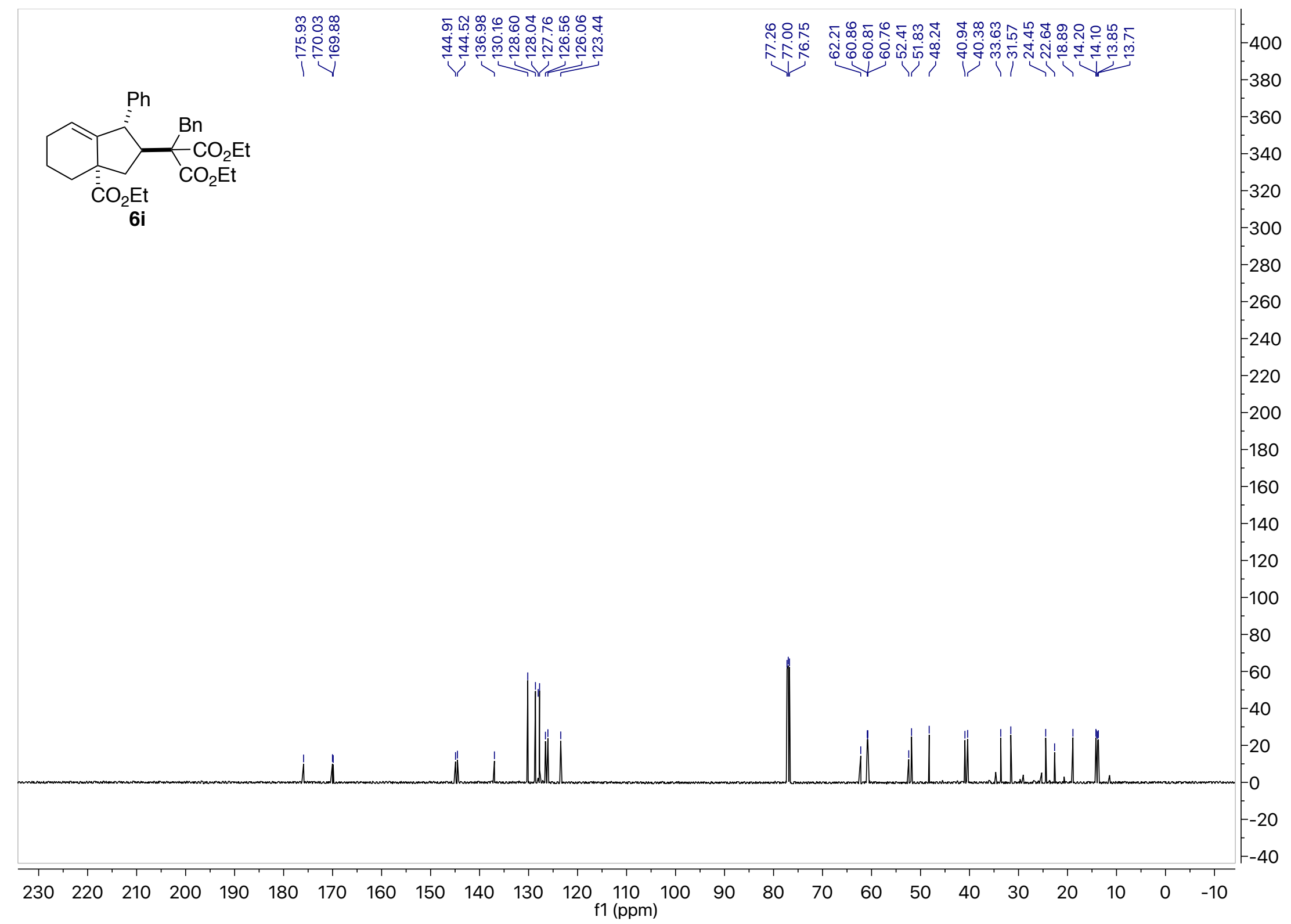




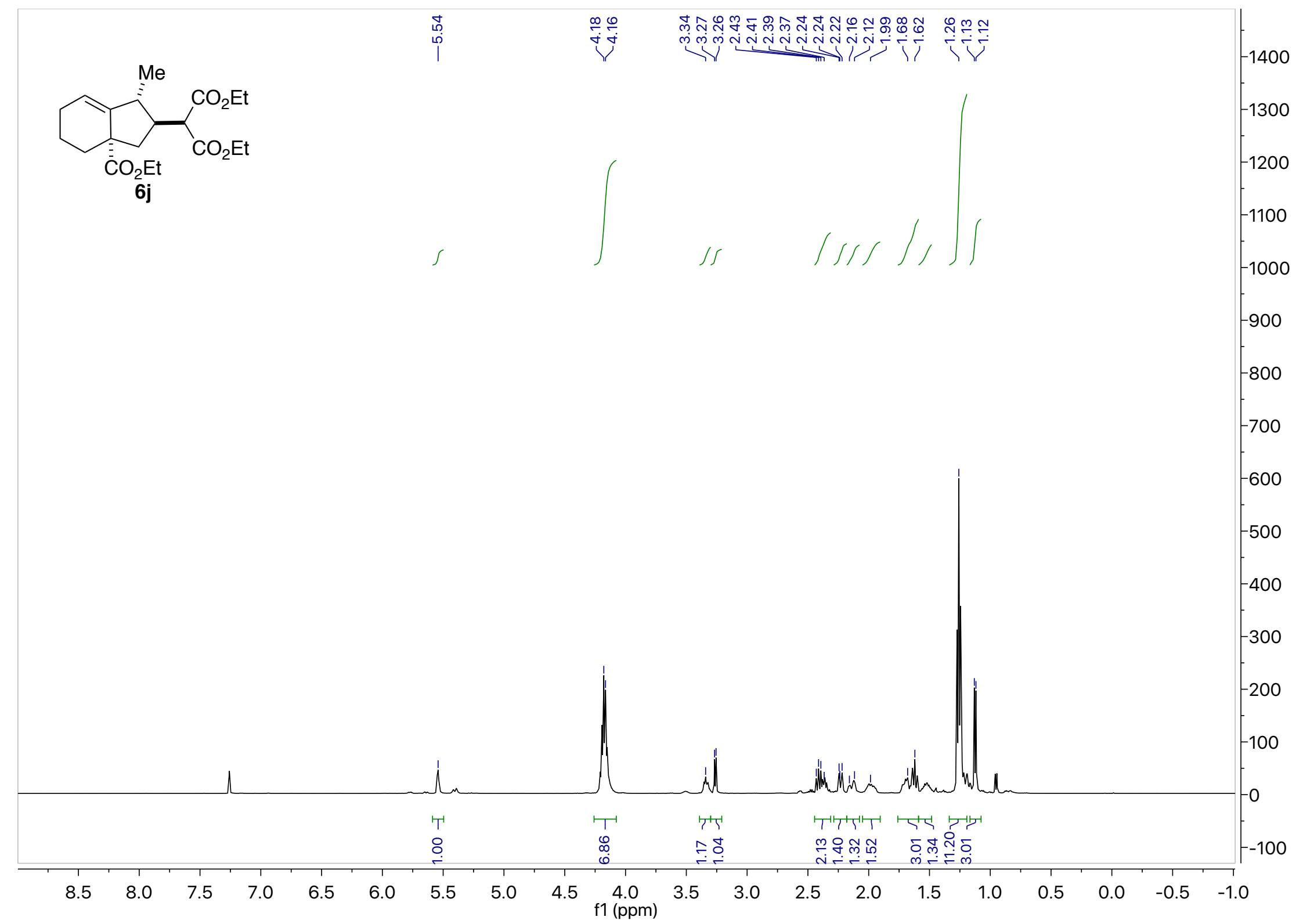




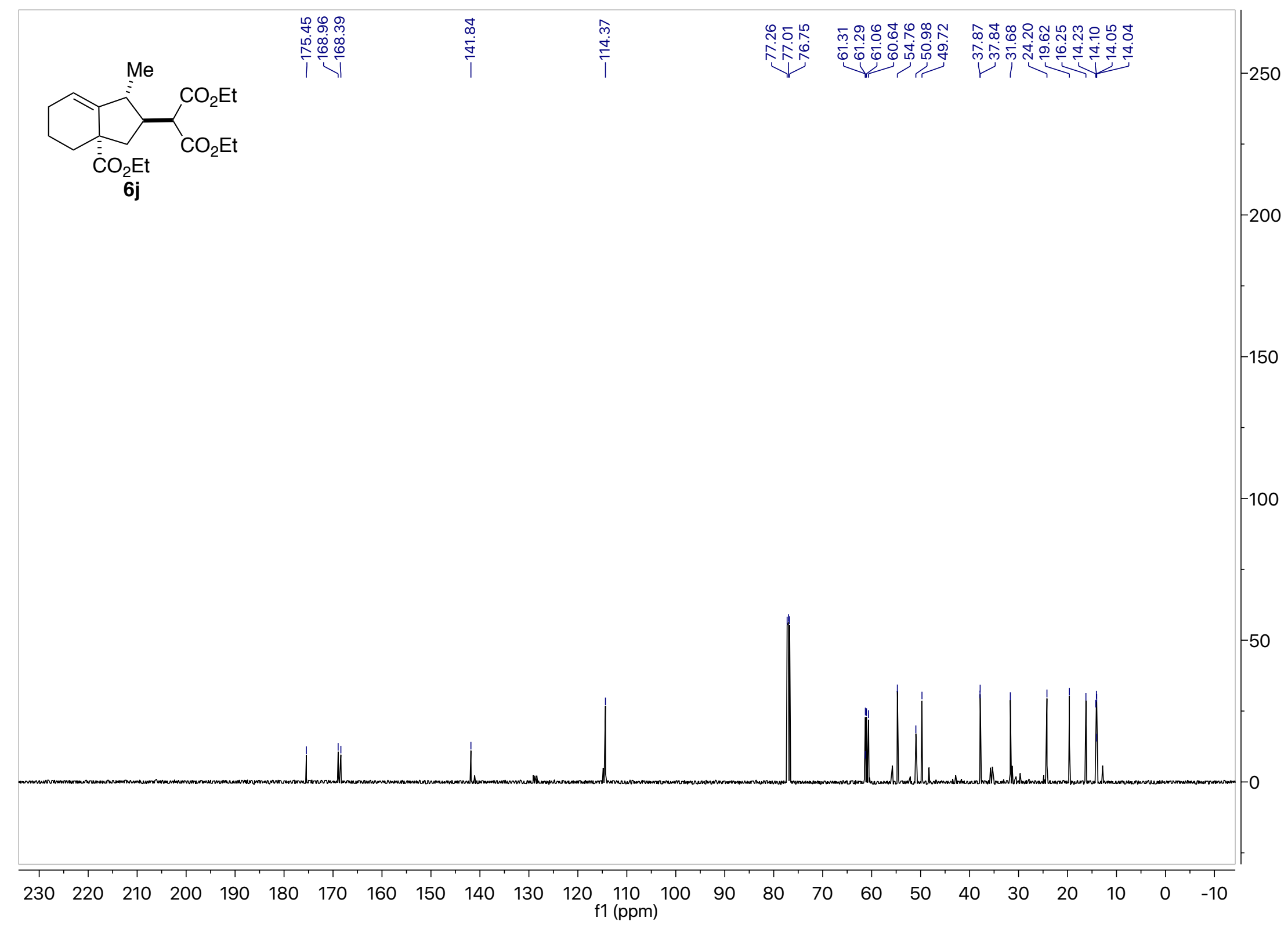




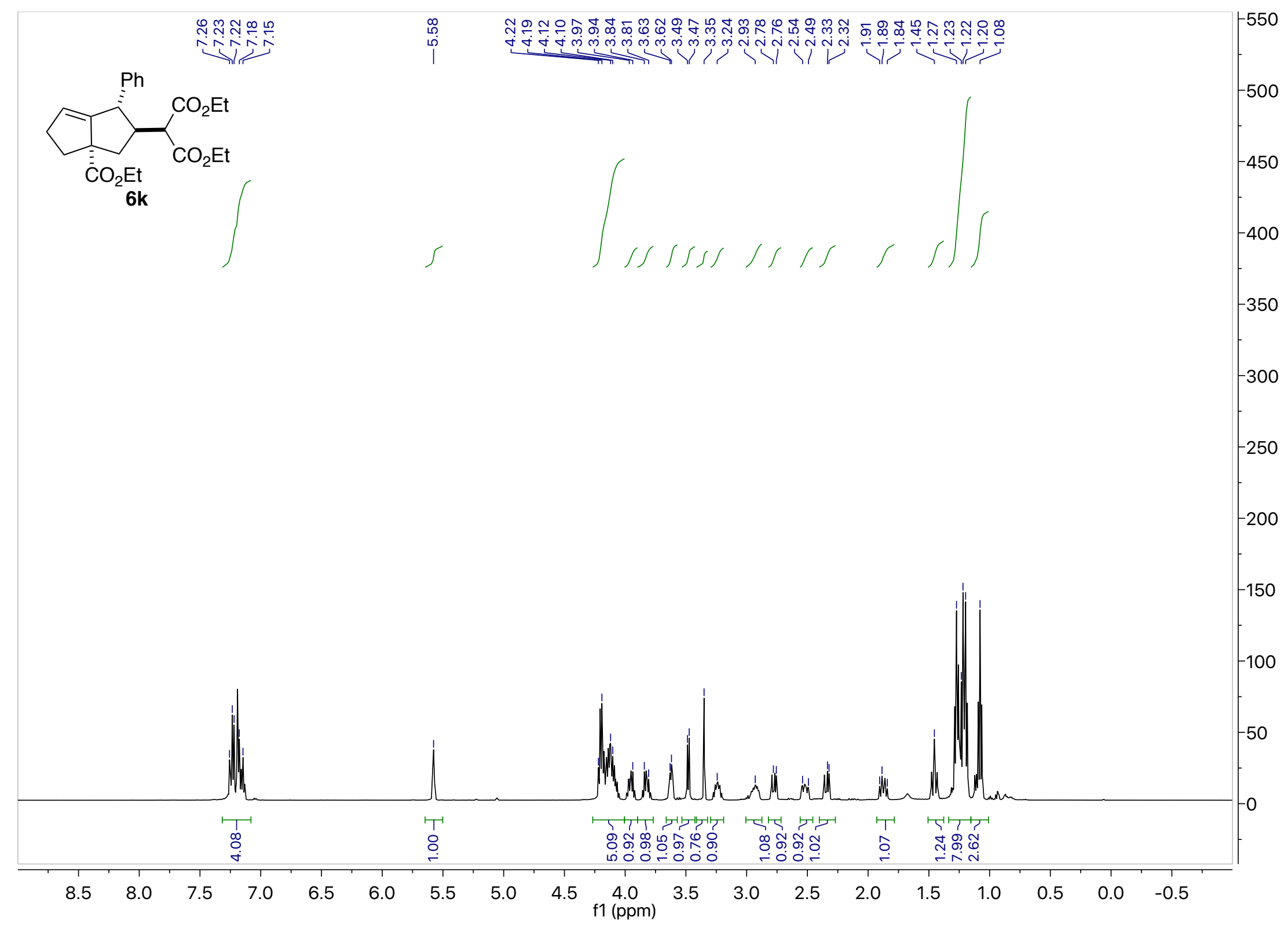




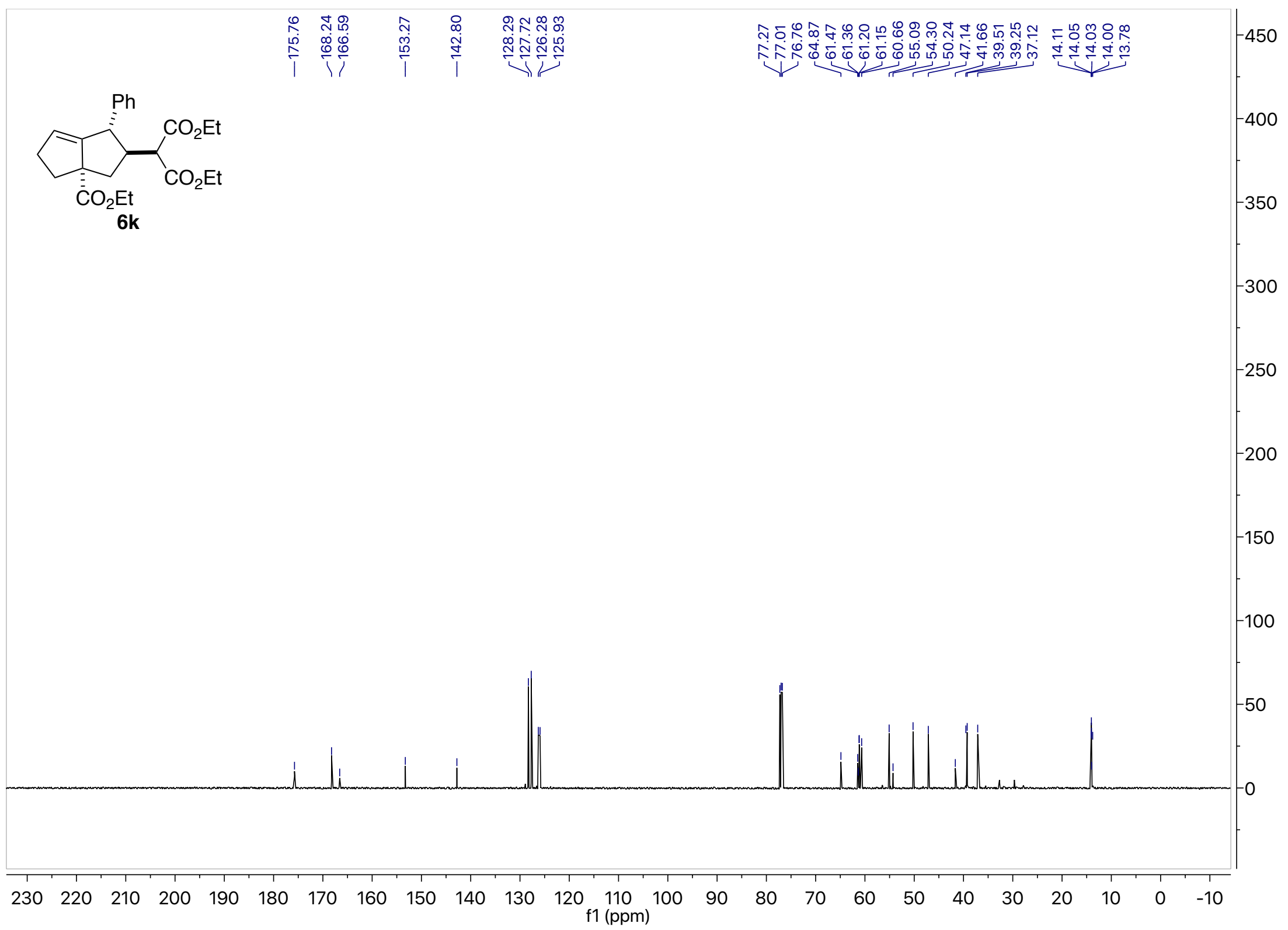




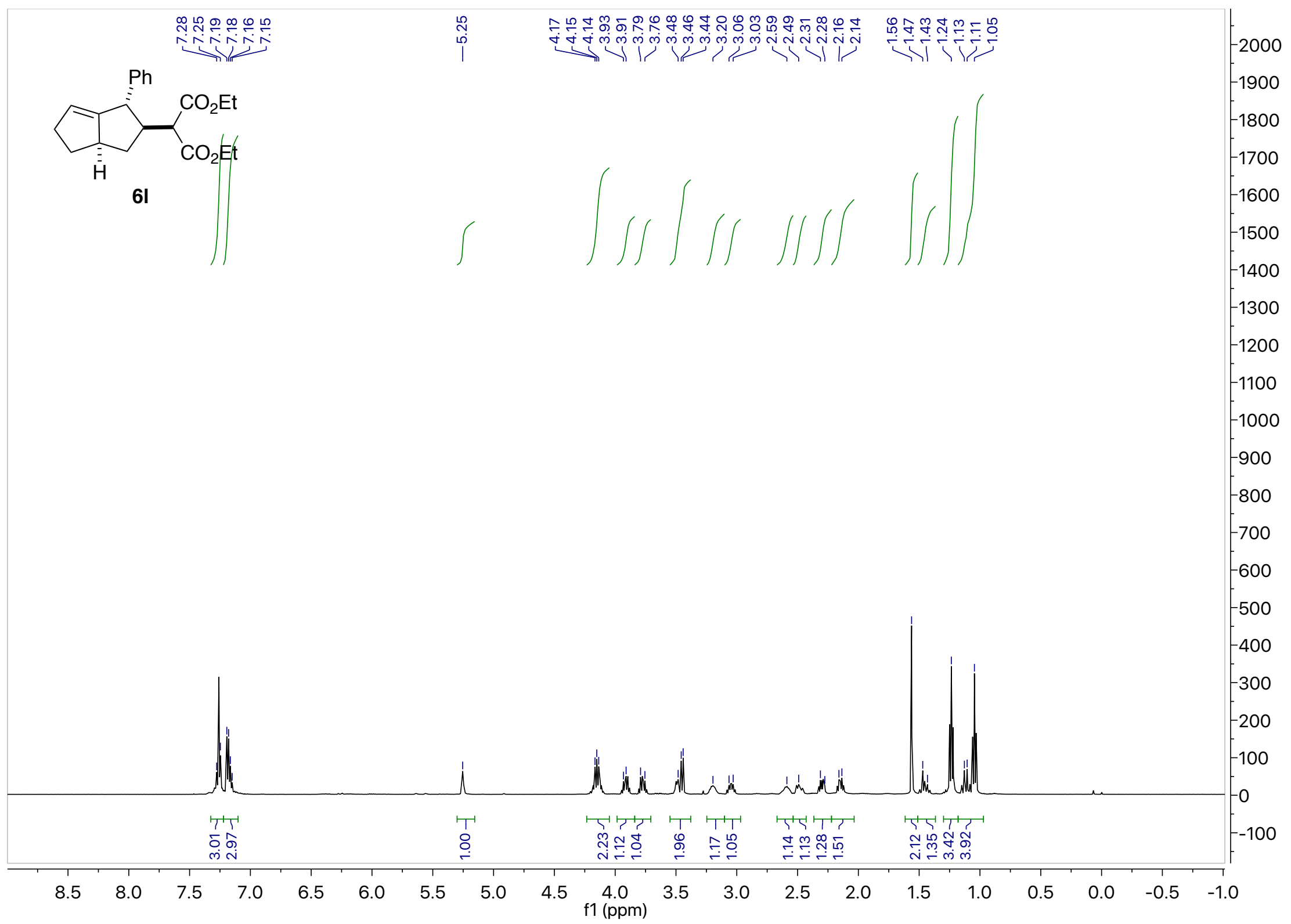




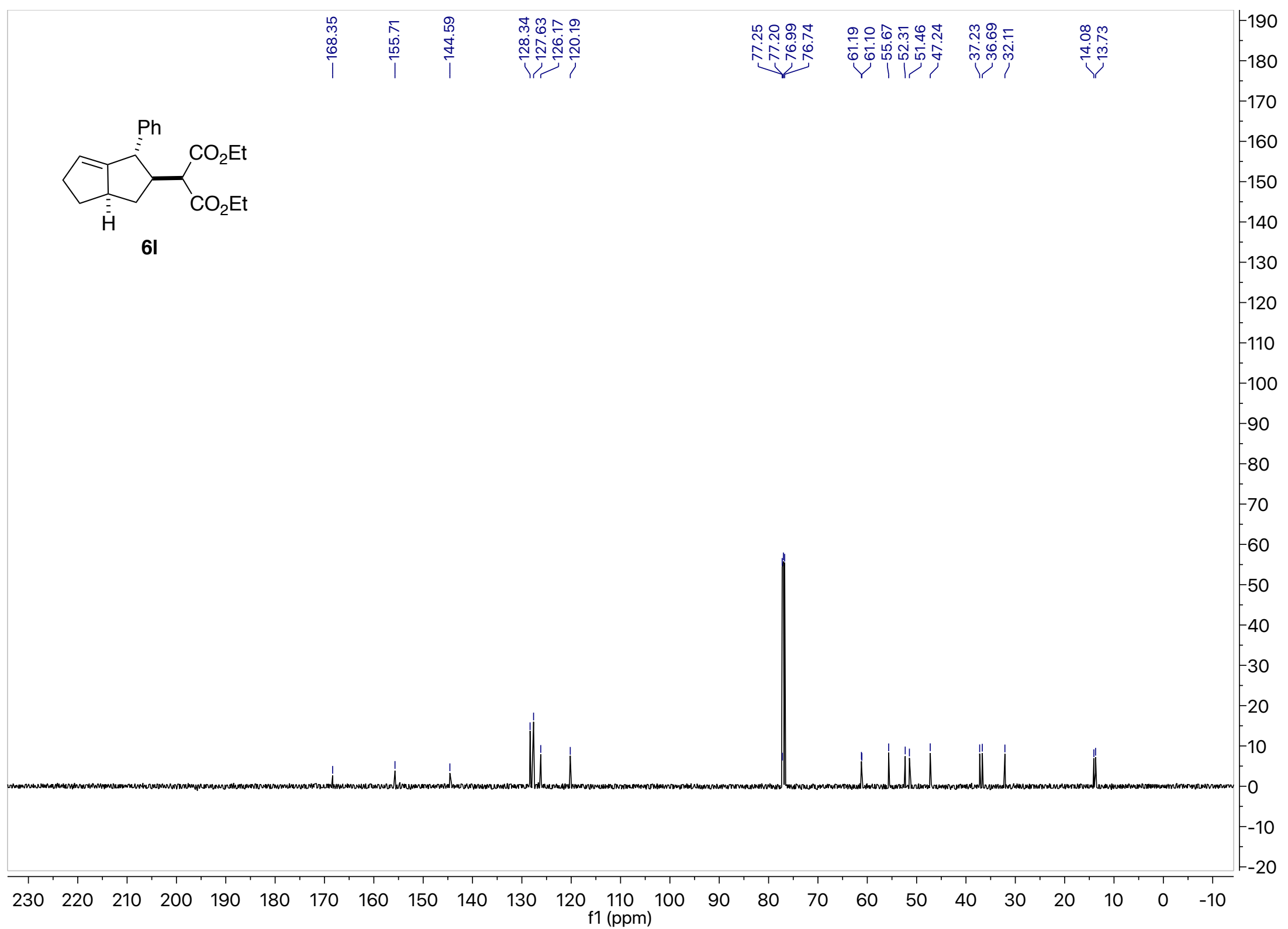




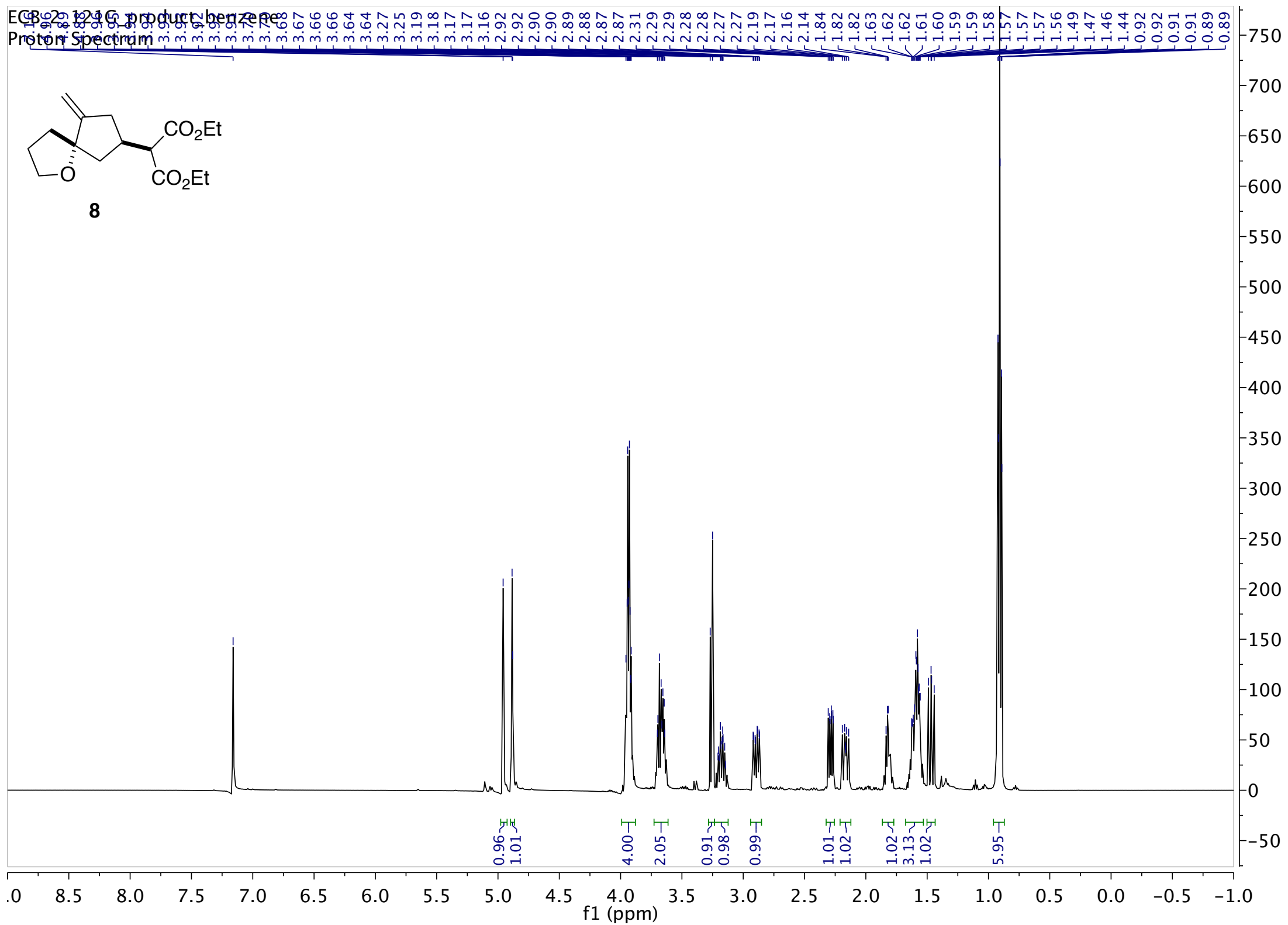




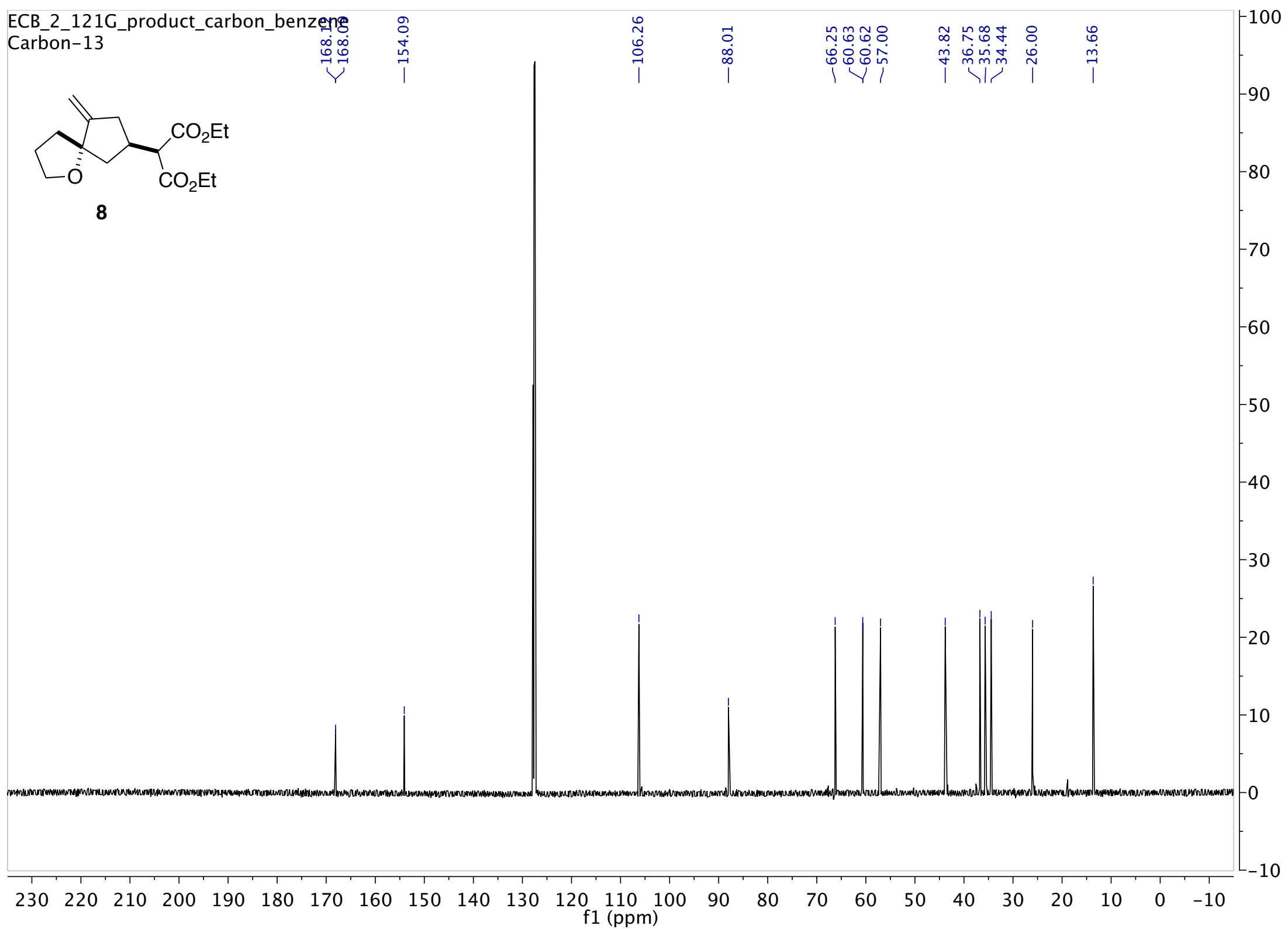




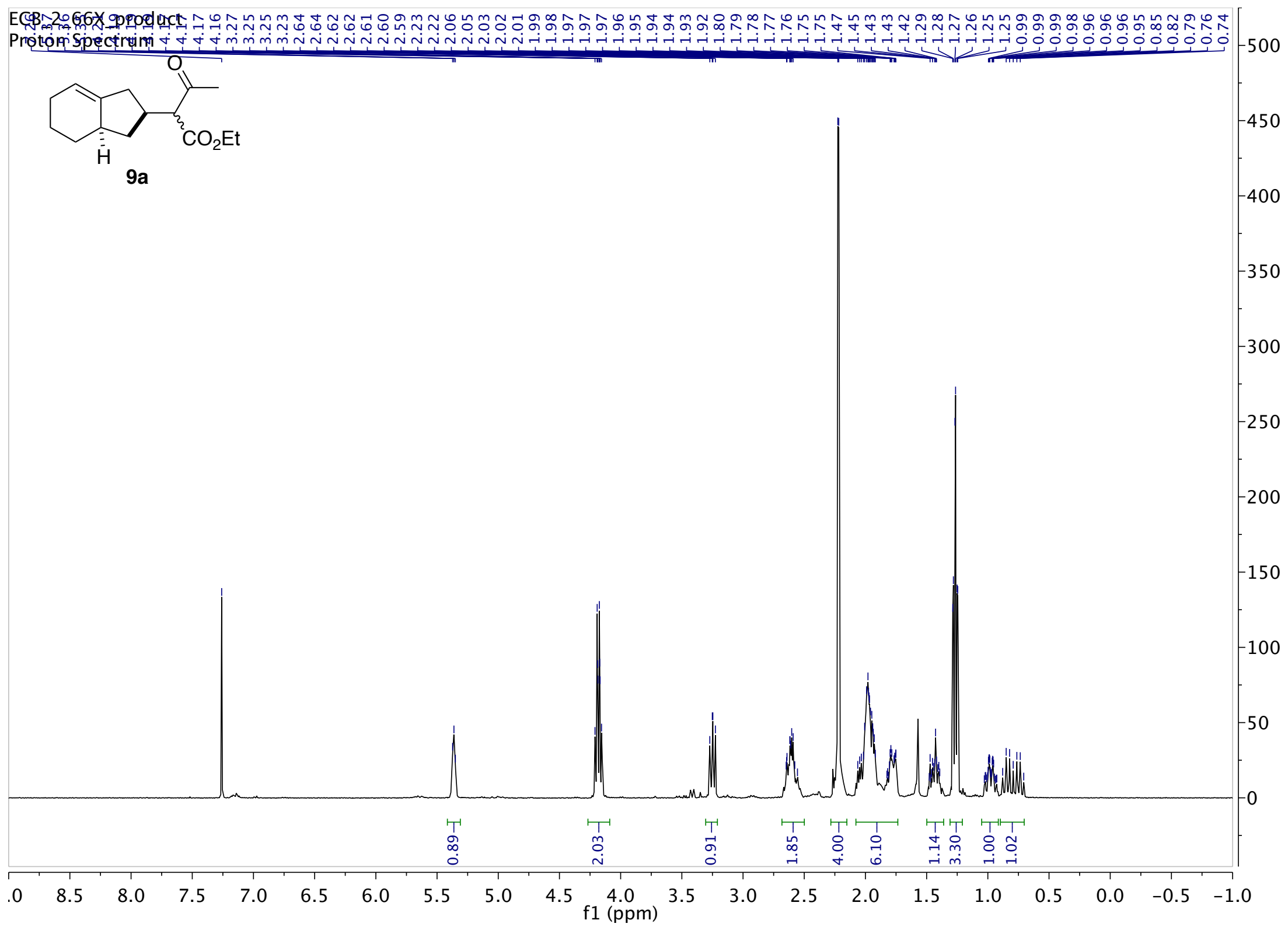




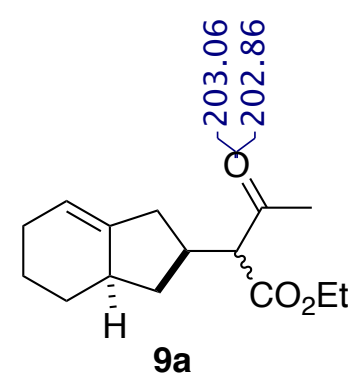

$m \stackrel{m}{m} \quad$ ํํำ

$\stackrel{n}{\sim}$ ำ

ชิำ

辛先

蚉

ᄀ부에

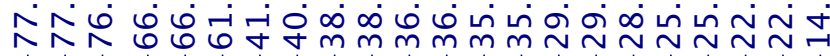

$9 a$

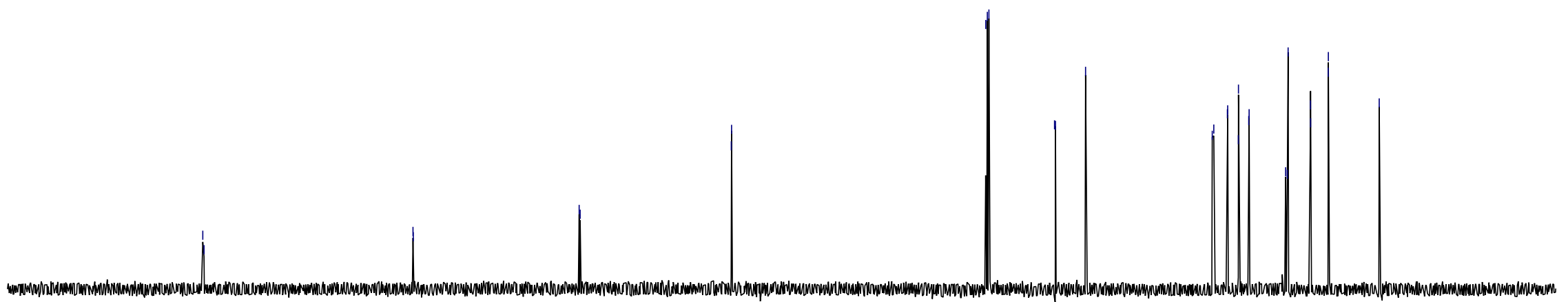

230

$\begin{array}{lll}150 & 140\end{array}$

$\begin{array}{llll}130 & 120 & 110 & 100\end{array}$

$\mathrm{f} 1$ (ppm)

90

80

$70 \quad 60$

50

$40 \quad 30$

20

10

$-10$ 


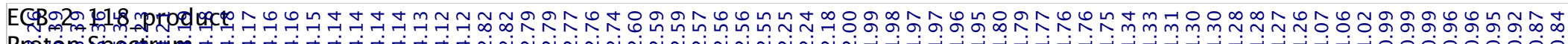

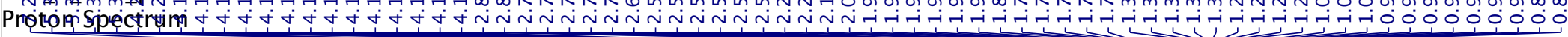

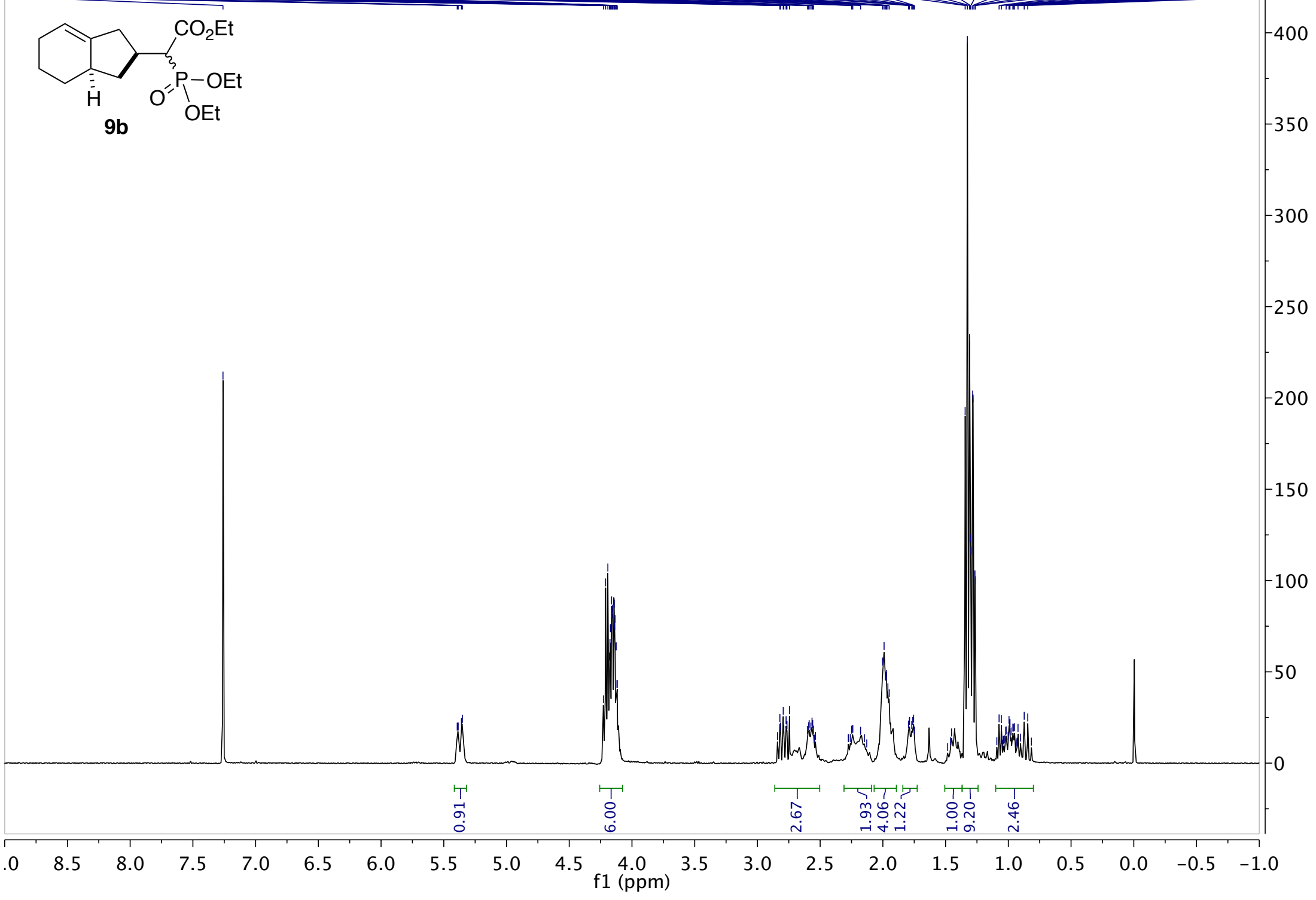




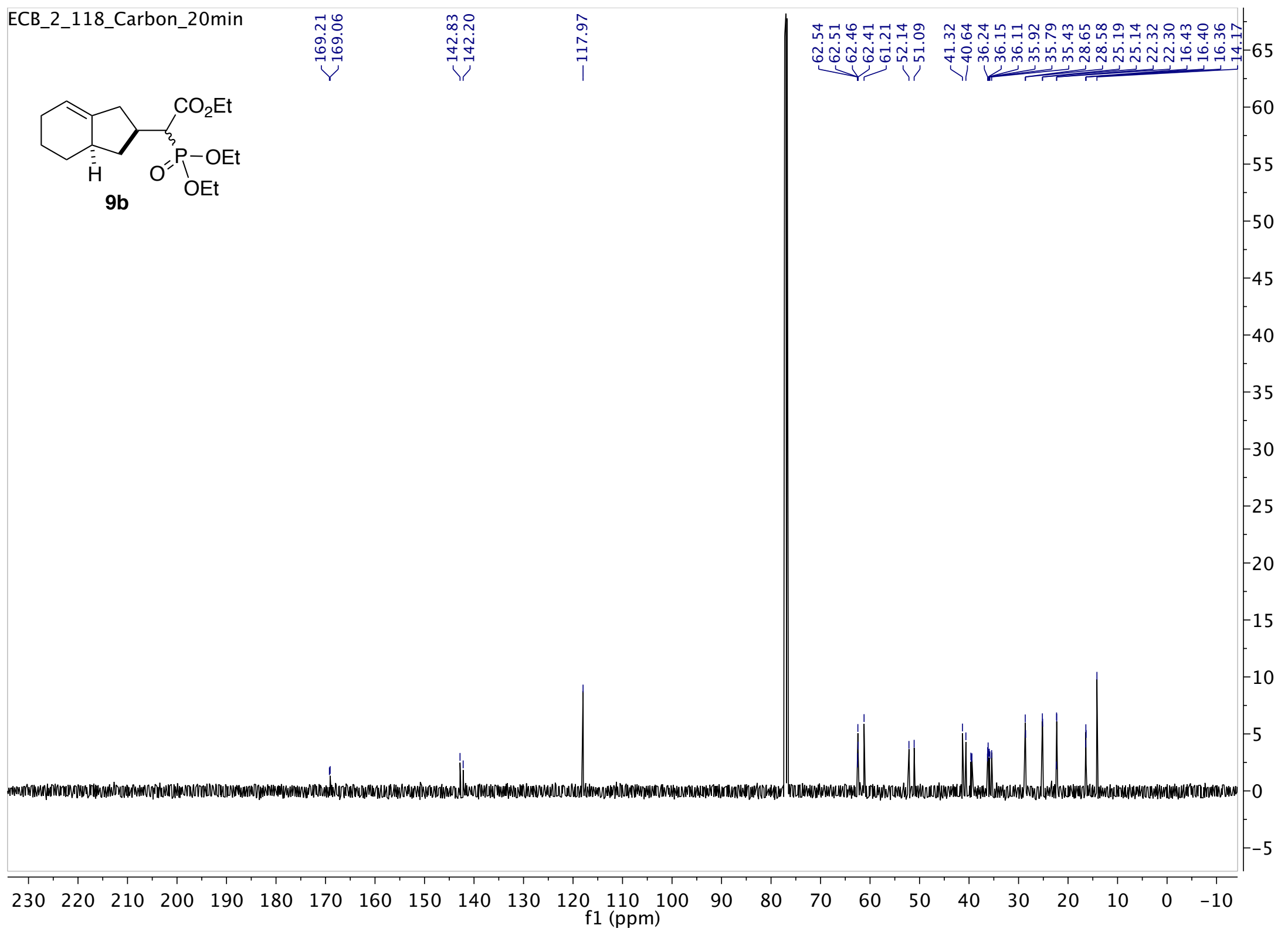




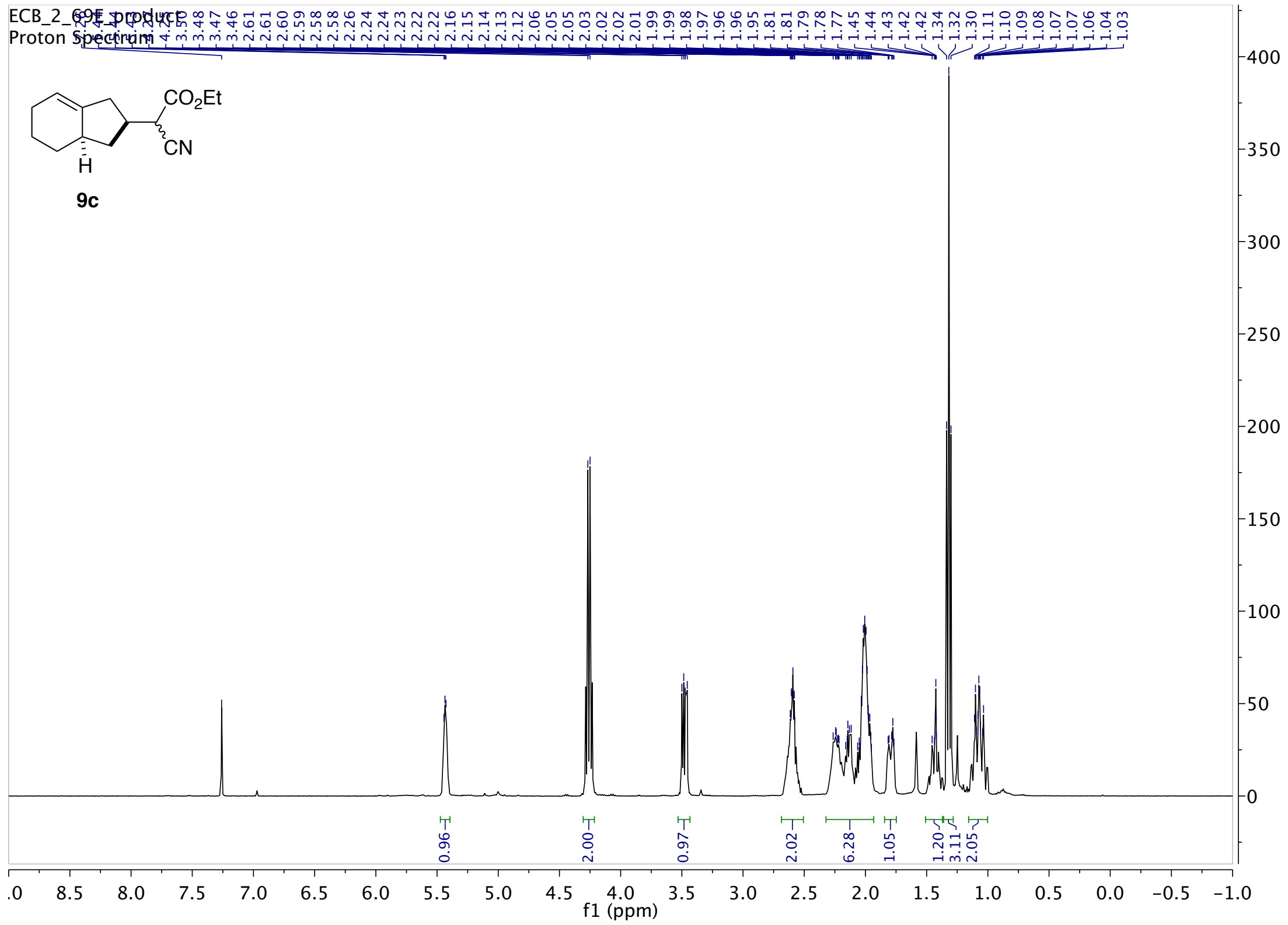




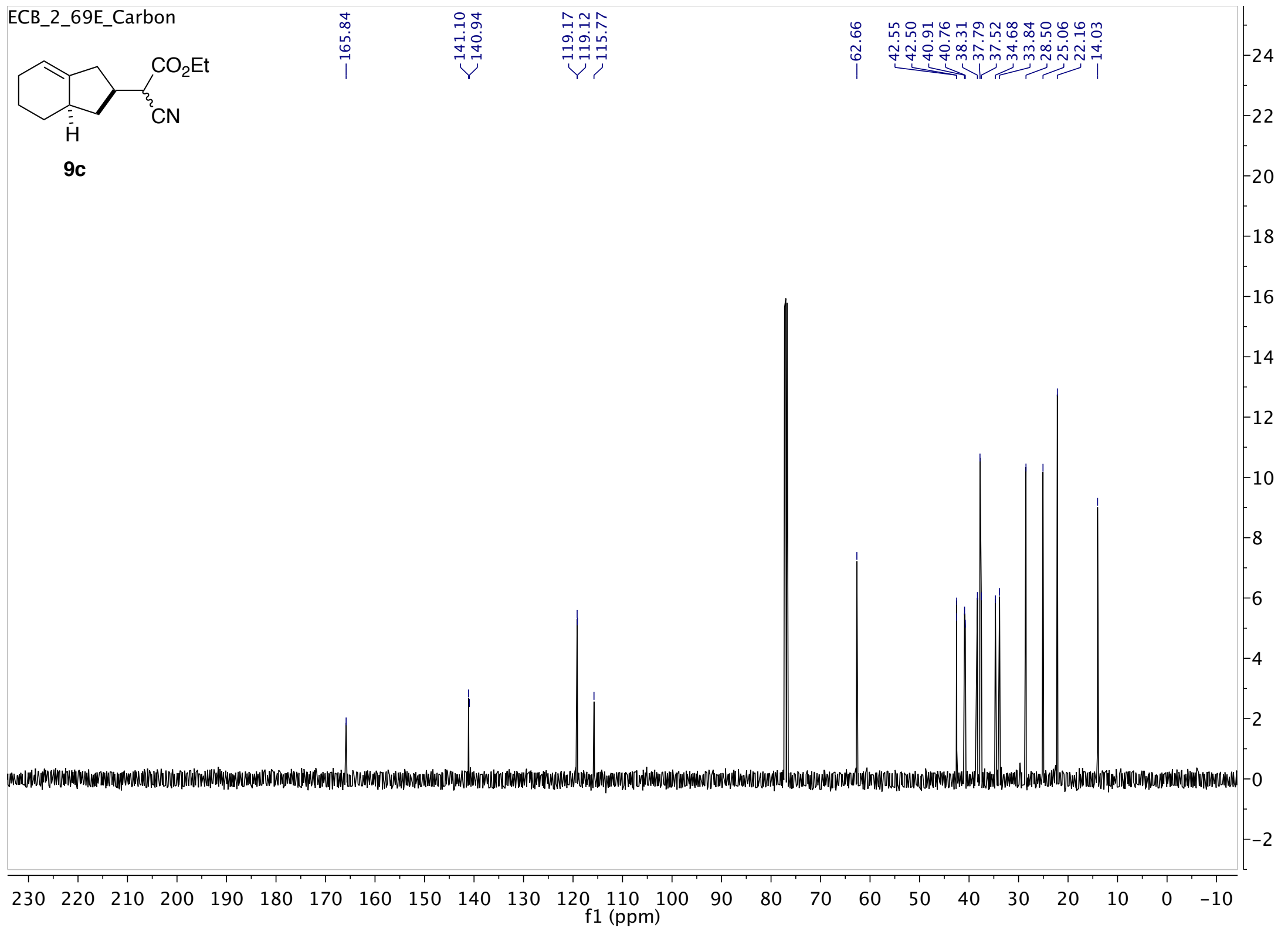

